\title{
DISCLAIMER
}

This report was prepared as an account of work sponsored by an agency of the United States Government. Neither the United States Government nor any agency thereof, nor any of their LBL- -29357 employes, makes any warranty, express or implied, or assumes any legal liability or responsibility for the accuracy, completeness, or usefulness of any information, apparatus, product, or process disclosed, or represents that its use would not infringe privately owned rights. Reference herein to any specific commercial product, process, or service by trade name, trademark, manufacturer, or otherwise does not necessarily constitute or imply its endorsement, recommendation, or favoring by the United States Governnent of any agency thereof. The views and opinions of authors expressed herein do not necessarily state of reflect those of the United States Government or any agency thereof.

\section{In-Beam Studies of High-Spin States of ACTINIDE NUCLEI}

\author{
Mark A. Stoyer \\ Ph. D. Thesis \\ Department of Chemistry \\ University of California \\ Berkeley, CA 94720 \\ and \\ Nuclear Science Division \\ Lawrence Berkeley Laboratory \\ Berkeley, CA 94720
}

Novernber 15, 1990

This work was supported in part by the Director, Office of Energy Research, Division of Nuclear Physics of the Office of High Energy and Nuclear Physics of the U.S. Department of Energy under Contract DE-AC03-76SF00098. 


\title{
In-Beam Studies of High-Spin States of Actinide Nuclei
}

\author{
by \\ Mark A. Stoyer
}

\begin{abstract}
A.bstract
High-spin states in the actinides have been studied using $\mathrm{C}$ ' llomb-excitation, inelastic excitation reactions, and one-neutron transfer reactions. Experimental data are presented for states in ${ }^{232} \mathrm{U},{ }^{233} \mathrm{U},{ }^{234} \mathrm{U},{ }^{235} \mathrm{U},{ }^{238} \mathrm{Pu}$ and ${ }^{239} \mathrm{Pu}$ from a variety of reactions. Energy levels, moments-of-inertia, aligned angular momentum, Routhians, gamma-ray intensities, and cross-sections are presented for most cases. Additional spectroscopic information (magnetic moments, $\frac{M 1}{E 2}$ mixing ratios, and g-factors) is presented for ${ }^{233} \mathrm{U}$. One- and two--eutron transfer reaction mechanisms and the possibility of band crossings ackbending) are discussed. A discussion of odd-A band fitting and Crankine calculations is presented to aid in the interpretation of rotational energy levels and alignment. In addition, several theoretical calculations of rotational populations for inelastic excitation and neutron transfer are compared to the data. Intratheory comparisons between the Sudden Approximation, Semi- Classical, and Alder-Winther-DeBoer methods are made. In connection with the theory development, the possible signature for the nuclear SQUID effect is discussed.
\end{abstract}




\section{Dedication}

This thesis would have been impossible without the support of the following people:

(1) To all those many people l've had the fortune of interacting with during my learning experiences while performing this and related work-too many to acknowledge individually, but certainly not forgotten.

(2) To Dr. Richard A. Meyer who welcomed me to my first job in Nuclear Chemistry, pointed me in the right direction at Berkeley, and whom I consider a good friend.

(3) To my brother, Dale, who first provoked in me the curiousity of a scientist; to my new sister-in-law, Janice, whose only connection with this thesis was a concern that I not recite it at her wedding; and to my parents, Lyn and Dorothy, who, though it is probably still somewhat of a mystery to them, nevertheless gave me the support and encouragement to "do whatever it is I do".

(4) And lastly, to Dr. John Rasmussen, whom it has been an honor to work for duiing my graduate studies. His patience, kindness, creativity, and guidance have been generous during my stay at LBL, and I am eternally in his debt. 


\section{Acknowledgements}

The author would like to acknowledge the Dr. John Rasmussen and Dr. Ken Crowe group (Dr. Roy Bossingham, Dr. Helmut Bossy, Dr. Felipe Canto $^{\dagger}$, Dr. Dean Chacon, Dr. Raul Donangelo ${ }^{\dagger}$, Dr. Aminah Farhan', Richard Hodge, Marvin Justice, Dr. Peter Ring ${ }^{\dagger}$, Dr. Quiyun Shao ${ }^{\dagger}$, Dr. Adnan Shihab-Eldin ${ }^{\dagger}$, Krysta Wyatt, Dr. Yongwen $\mathrm{Xu}^{\dagger}$, Dr. Yanglin $\mathrm{Ye}^{\dagger}$ ), the Dr. Doug Cline group at the University of Rochester (Karl Helmer, Dr. Alex Kavka, Dr. Warnick Kernan, Ehrenfried Vogt, Dr. Ching Yen Wu), and the Dr. Mike Guidry group at ORNL/University of Tennessee (Wayne Kincaid, Dr. Xin Tao Liu) for help with the experimental aspects of this thesis. The author would also like to acknowledge Dr. Richard A. Meyer, Dr. Dan Decman, Dr. Eugene Henry, and Dr. Wolfgang Stöffl of LLNL for help with the spectral fitting software, and the Dr. Darleane Hoffman group at LBL (Dr. Diane Bennett, Carolyn Gannett, Dr. Ken Gregorich, Dr. Howard Hall, Dr. Roger Henderson, Dr. John Leyba) as well as Ken Biscay and Glenn Garabedian of EH\&S for help with the target making.

This work was supported in part by the Director, Office of High Energy Research, Division of Nuclear Physics of the Office of High Energy and Nuclear Physics of the U.S. Department of Energy under Contract DE-AC03T6SE00098.

\footnotetext{
IVisiting scholar
} 


\section{Contents}

A. Experimental ..................... 1

1 Introduction $\quad 1$

2 Experimental Methods 5

2.1 General Description .................. 5

$2.1 .1{ }^{58} \mathrm{Ni}+{ }^{235} \mathrm{U}$ Reaction at ORNL .......... 13

$2.1 .2{ }^{206} \mathrm{~Pb}+{ }^{233} \mathrm{U}$ Reaction at LBL . . . . . . . . . 17

$2.1 .3{ }^{58} \mathrm{Ni}+{ }^{233} \mathrm{U}$ Reaction at UOR ........... 20

$2.1 .4{ }^{90} \mathrm{Zr}+{ }^{239} \mathrm{Pu}$ Reaction at ORNL . . . . . . . . . 20

2.2 Data Analysis Techniques .............. 24

3 Results and Discussion 33

$3.1{ }^{58} \mathrm{Ni}+{ }^{235} \mathrm{U}$ Reaction $\ldots \ldots \ldots 33$

$3.2{ }^{206} \mathrm{~Pb}+{ }^{233} \mathrm{U}$ and ${ }^{58} \mathrm{Ni}+{ }^{233} \mathrm{U}$ Reactions .......... 39

$3.3{ }^{90} \mathrm{Zr}+{ }^{239} \mathrm{Pu}$ Reaction ................ 72

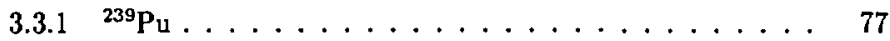

$3.3 .2{ }^{238} \mathrm{Pu} \ldots \ldots \ldots \ldots \ldots$

B. Theoretical .......................... 116

4 Introduction $\quad 116$

5 Discussion of Relevant Theories $\mathbf{1 2 0}$

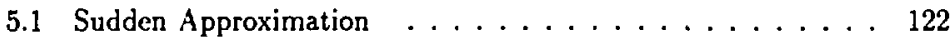

5.2 Alder-Winther-deBoer modified method . . . . . . . . 128

5.2 .1 General Description . . . . . . . . . . . . . 128 
5.2 .2 Coulomb Excitation $\ldots \ldots \ldots \ldots \ldots$

5.2 .3 Inelastic excitation . . . . . . . . . . . . 132

5.2.4 Neutron Transfer . . . . . . . . . . . . . . 135

6 Comparison of Theory with Experimental Data 144

6.1 Brief SQUID Results . . . . . . . . . . . . . . . . 145

$6.2{ }^{233} \mathrm{U}$ Results $\ldots \ldots \ldots \ldots \ldots \ldots \ldots \ldots \ldots \ldots \ldots$

$6.3{ }^{90} \mathrm{Zr}+{ }^{239} \mathrm{Pu}$ Results .................. 158

$6.3 .1{ }^{239} \mathrm{Pu}$ Inelastic Excitation . . . . . . . . . . 158

$6.3 .2{ }^{238} \mathrm{Pu}$ One-Neutron Transfer Results ........ 166

7 Conclusions $\quad 179$

A Target Construction 183

A.1 General Apparatus . . . . . . . . . . . . . 183

A.2 ${ }^{233} \mathrm{U}$ Target Preparation . . . . . . . . . . . . . 186

A.3 ${ }^{239} \mathrm{Pu}$ Target Preparation . . . . . . . . . . . . 187

$\begin{array}{ll}\text { B FITEK Program } & 189\end{array}$ 


\section{List of Figures}

2.1 Schematic diagram of generic experimental configuration. $\quad \ldots 6$

2.2 Calibration of particle scattering angles in a PPAC. . . . 10

2.3 Diagram of ${ }^{58} \mathrm{Ni}+{ }^{235} \mathrm{U}$ experimental setup. . . . . . . . 14

2.4 Diagram of ${ }^{206} \mathrm{~Pb}+{ }^{233} \mathrm{U}$ experimental setup. . . . . . . 18

2.5 Diagram of ${ }^{90} \mathrm{Zr}+{ }^{239} \mathrm{Pu}$ experimental setup. . . . . . . 22

2.6 Mass spectrum from one PPAC for the ${ }^{206} \mathrm{~Pb}+{ }^{233} \mathrm{U}$ reaction. 27

$2.7{ }^{152}$ Eu calibration spectrum for a Ge detector. . . . . . . 28

2.8 The top figure is an uncorrected $\mathrm{Ge} E_{\gamma}$ spectrum. The middle figure is the same Ge $E_{\gamma}$ spectrum with Doppler corrections, and the bottom figure is the Ge timing spectrum. . . . . . 30

$2.9 \mathrm{Nal}(\mathrm{Tl})$ timing spectrum showing the neutron- $\gamma$-ray separation for the ${ }^{90} \mathrm{Zr}+{ }^{239} \mathrm{Pu}$ reaction for events depositing $2-16 \mathrm{MeV}$ in the detector. . . . . . . . . . . . . 31

3.1 Ge $\gamma$-ray spectrum for the ${ }^{58} \mathrm{Ni}+{ }^{235} \mathrm{U}$ reaction $\ldots \ldots \ldots \quad 35$

3.2 Total $E, M$ plot for the ${ }^{58} \mathrm{Ni}+{ }^{235} \mathrm{U}$ reaction where the dashed line indicates the approximate Yrast line for ${ }^{234} \mathrm{U}$ assuming no excitation of the ${ }^{58} \mathrm{Ni} . \ldots \ldots \ldots \ldots \ldots \ldots$

3.3 Total $\gamma$-ray spectrum for the ${ }^{206} \mathrm{~Pb}+{ }^{233} \mathrm{U}$ reaction $\ldots \ldots .40$

3.4 Level diagram for both signatures of the ground-state band in



3.5 Moments-of-inertia for ground-state band in ${ }^{233} \mathrm{U} \ldots \ldots . . .44$

3.6 Plot of $\frac{E_{I}-E_{I-1}}{2 I}$, the inverse moment-of-inertia $\left(\frac{\hbar^{2}}{2 S^{(1)}}\right)$ for $\Delta I=1$ transitions, vs. $2 I^{2}$ showing the magritude of the staggeting term. 
3.7 A comparison between experimental energy levels and theoretical energy levels for the ground rotational band in ${ }^{233} U$. The theoretical energy levels are calculated using the cranking model described in the text and the standard Kumar and Baranger pa-

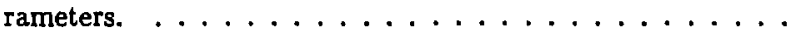

3.8 A comparison of extracted aligned angular momentum for the ground rotational band in ${ }^{233} \mathrm{U}$ between experiment and cranking model calculations . . . . . . . . . . . . .

3.9 Experimental Routhians for the ground-state band in ${ }^{233} \mathrm{U}$. . .

3.10 Routhians from cranking calculation for the ground-state band in ${ }^{233} \mathrm{U}$.

3.11 Routhians from cranking calculation for the ground-state band in ${ }^{233} \mathrm{U}$ including a slight $\gamma$-deformation.

3.12 Total Ge $\gamma$-ray spectrum for the ${ }^{90} \mathrm{Zr}+{ }^{239} \mathrm{Pu}$ reaction. . . . . 73

3.13 Neutron multiplicity for the ${ }^{90} \mathrm{Zr}+{ }^{239} \mathrm{Pu}$ reaction.

3.14 Total two dimensional Ge $\gamma-\gamma$ coincidence spectrum for the ${ }^{90} \mathrm{Zr}$ $+{ }^{239} \mathrm{Pu}$ reaction showing relationships between $\gamma$-rays in this experiment. Both inelastic ${ }^{239} \mathrm{Pu} \gamma$-rays and one-neutron transfer ${ }^{238} \mathrm{Pu} \gamma$-rays are present. . . . . . . . . . . . . . . .

3.15 Total $(H, K)$ spectrum for the ${ }^{90} \mathrm{Zr}+{ }^{239} \mathrm{Pu}$ reaction. . . . . 76

3.16 Projection of a) $H$ and b) $K$ for the ${ }^{90} \mathrm{Zr}+{ }^{239} \mathrm{Pu}$ reaction. . 78

$3.17 \mathrm{Ge} \gamma-\gamma$ spectrum for the ${ }^{90} \mathrm{Zr}+{ }^{239} \mathrm{Pu}$ reaction gated on the $201.1-\mathrm{keV} \frac{17}{2}^{+} \rightarrow \frac{13}{2}^{+}, 212.1-\mathrm{keV} \frac{19}{2}^{+} \rightarrow \frac{15}{2}^{+}, 288.3-\mathrm{keV} \frac{25}{2}^{+} \rightarrow$ $\frac{21}{2}^{+}$and $299.7-\mathrm{keV}^{2} \frac{27}{2}^{+} \rightarrow \frac{23}{2}^{+}$transitions.

3.18 Moments-of-inertia for the ground-state band in ${ }^{239} \mathrm{Pu}$.

3.19 Inverse moment-of-inertia, $\Im^{(1)}$, for the ground-state band in ${ }^{239} \mathrm{Pu}$ showing the magnitude of the staggering between the favored and unfavored signatures.

3.20 Aligned angular momentum $\left(i_{a}\right)$ for the ground-state band in ${ }^{239} \mathrm{Pu}$.

3.21 Experimental Routhians for the ground-state band in ${ }^{239} \mathrm{Pu} . \quad . \quad 87$

3.22 Theoretical Routhians for ${ }^{239} \mathrm{Pu}$ as a function of $\omega . \ldots 88$

3.23 Inelastic $(H, K)$ spectrum for the ${ }^{90} \mathrm{Zr}+{ }^{239} \mathrm{Pu}$ reaction. . . 90

3.24 Projections of a) $H$ and b) $K$ for the ${ }^{90} \mathrm{Zr}+{ }^{239} \mathrm{Pu}$ reaction. . 
3.25 The $\gamma$-ray spectrum gated on three known ${ }^{238} \mathrm{Pu}$ transitions, the $307-\mathrm{keV} 12^{+} \rightarrow 10^{+}, 349-\mathrm{keV} 14^{+} \rightarrow 12^{+}$and $389-\mathrm{keV}$ $16^{+} \rightarrow 14^{+}$transitions for the one-neutron transfer reaction.

3.26 Kinematic (open circles) and dynamic (•) moments-of-inertia for the ground-state band in ${ }^{238} \mathrm{Pu} . \ldots \ldots \ldots \ldots$. . . . . 94

3.27 Experimental Routhians for ${ }^{238} \mathrm{Pu} . \ldots \ldots \ldots \ldots \ldots$

3.28 Transfer $(H, K)$ spectrum for ${ }^{238} \mathrm{Pu} . \ldots \ldots \ldots \ldots . \ldots 9$

3.29 Projections of a) $H$ and b) $K$ for the transfer reaction. . . . $\quad 99$

3.30 Differential cross-sections for one-neutron transfer as a function of distance of closest approach in the lab. . . . . . . . 102

3.31 Differential cross-sections for one-neuiron transfer as a function of distance of closest approach in the center-of-mass frame. . 103

3.32 Reduced differential cross-sections for one-neutron transfer as a function of distance of closest approach in the lab. . . . . . . 104

3.33 One-neutron transfer probability as a function of distance of closest approach. The solid line represents a simple theory based on equation (3.34).

$3.34 \mathrm{Ge} \gamma$-ray spectrum gated on the "mystery" transition at 145 keV in the ${ }^{90} \mathrm{Zr}+{ }^{239} \mathrm{Pu}$ experiment. . . . . . . . . . 108

$3.35 \mathrm{Ge} \gamma$-ray spectrum gated on the "mystery" transition at 185 $\mathrm{keV}$ in the ${ }^{90} \mathrm{Zr}+{ }^{239} \mathrm{Pu}$ experiment. . . . . . . . . . 109

3.36 Ge $\gamma$-ray spectrum gated on the "mystery" transition at 226 keV in the ${ }^{90} \mathrm{Zr}+{ }^{239} \mathrm{Pu}$ experiment. . . . . . . . . . 110

3.3i Ge $\gamma$-ray spectrum gated on the "mystery" transition at 268 $\mathrm{keV}$ in the ${ }^{90} \mathrm{Zr}+{ }^{239} \mathrm{Pu}$ experiment. . . . . . . . . 111

5.1 Definition of geometry of systems under investigation. . . . 121

6.1 Theoretical calculation of the rotational population probability for states in the ground band in the neutron pair transfer reaction ${ }^{208} \mathrm{~Pb}+{ }^{160}$ Dy reaction. . . . . . . . . . . . 148

6.2 Theoretical calculation of the rotational population probability for states in the ground band in the neutron pair transfer reaction ${ }^{208} \mathrm{~Pb}+{ }^{158}$ Dy reaction. . . . . . . . . . . . 149 
6.3 A comparison of the experimental rotational population yields extracted from the reaction ${ }^{156} \mathrm{Gd}+{ }^{206} \mathrm{~Pb}$ with the calculations of theory from Section 5.2.4 for the reactions ${ }^{208} \mathrm{~Pb}+{ }^{160} \mathrm{Dy}$ and ${ }^{208} \mathrm{~Pb}+{ }^{158} \mathrm{Dy} \ldots \ldots \ldots \ldots \ldots \ldots$

6.4 Rotational population pattern for the ${ }^{58} \mathrm{Ni}+{ }^{233} \mathrm{U}$ Coulomb excitation experiment. . . . . . . . . . . . . 153

6.5 Rotational population pattern for the ${ }^{206} \mathrm{~Pb}+{ }^{233} \mathrm{U}$ inelastic excitation experiment. . . . . . . . . . . 156

6.6 Inelastic excitation rotational population pattern in the ground

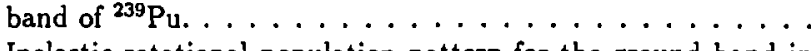

6.7 Inelastic rotational population pattern for the ground band in ${ }^{239} \mathrm{Pu}$ extracted from the $\gamma-\gamma$ coincidence spectrum gated on Yrast transitions.

6.8 Theoretical calculation of rotational popuiation probability for the inelastic excitation experiment ${ }^{90} \mathrm{Zr}+{ }^{239} \mathrm{Pu}$.

6.9 Rotational population pattern for the states populated in ${ }^{238} \mathrm{Pu}$ in the one-neutron transfer reaction.

6.10 Several theoretical calculations of the rotational population patterns in ${ }^{238} \mathrm{Pu}$ from one-neutron transfer using deformation $\eta=6.170$

6.11 Same as Figure 6.10 but for deformation $\eta=4$. Pure Coulomb excitation is not shown in this figure.

6.12 Best theoretical calculation of the rotational population patterns in ${ }^{238} \mathrm{Pu}$ from one-neutron transfer. . . . . . . . . . 172

6.13 Parts of the surface form factor $F_{\text {II }}$ for ${ }^{239} \mathrm{Pu}$. . . . . . . . 176

6.14 Comparison of the best theoretical calculation of the rotational population patterns in ${ }^{238} \mathrm{Pu}$ from one-neutron transfer with experimental data. .................. 177

A.1 Schematic of electrodeposition apparatus. . . . . . . . . 184 


\section{List of Tables}

2.1 Isotopic composition of target for ${ }^{58} \mathrm{Ni}+{ }^{20} \mathrm{U}$ experiment $\ldots \quad 15$

2.2 Lab detector positions for ${ }^{58} \mathrm{Ni}+{ }^{235} \mathrm{U}$ experiment $\ldots \ldots .15$

2.3 Lab detector positions for ${ }^{58} \mathrm{Ni}+{ }^{235} \mathrm{U}$ experiment $\ldots \ldots . \quad 16$

2.4 Isotopic composition of target for ${ }^{206} \mathrm{~Pb}+{ }^{233} \mathrm{U}$ experiment . 17

2.5 Lab detector positions for ${ }^{206} \mathrm{~Pb}+{ }^{233} \mathrm{U}$ experiment $\ldots \ldots . \quad 19$

2.6 Isotopic composition of target for ${ }^{90} \mathrm{Zr}+{ }^{239} \mathrm{Pu}$ experiment . . 21

2.7 Lab detector positions for ${ }^{90} \mathrm{Zr}+{ }^{239} \mathrm{Pu}$ experiment $\ldots \ldots .23$

2.8 Lab-detector positions for ${ }^{90} \mathrm{Zr}+{ }^{239} \mathrm{Pu}$ experiment $\ldots \ldots .23$

3.1 Observed transitions in ${ }^{233} \mathrm{U} \ldots \ldots \ldots \ldots \ldots \ldots$

3.2 Orbitals used as basis for Cranking model calculations . . . 47

3.3 Main parts of the ground-state band wavefunction for ${ }^{233} \mathrm{U}$ in Cranking model $\ldots \ldots \ldots \ldots \ldots \ldots$. . . . . 50

$3.4{ }^{233} \mathrm{U}$ rotational band fit parameters $(\mathrm{keV}) \ldots \ldots \ldots \ldots . \quad \ldots 7$

3.5 Apparent $\mathrm{g}$-factors deduced from cascade-to-crossover transition ratios for ${ }^{233} \mathrm{U} \ldots \ldots \ldots \ldots \ldots \ldots \ldots \ldots \ldots$

3.6 Observed transitions in ${ }^{239} \mathrm{Pu} \ldots \ldots \ldots \ldots \ldots$. . . . . 81

3.7 Observed transitions in ${ }^{238} \mathrm{Pu} \ldots \ldots \ldots \ldots \ldots \ldots$

3.8 Peak assignments for the Ge $\gamma$-ray spectrum gated on the 268$\mathrm{keV}$ "mystery" transition observed in the ${ }^{90} \mathrm{Zr}+{ }^{239} \mathrm{Pu}$ experiment

6.1 Relative spot intensities for the probability of populating a given spin in ${ }^{239} \mathrm{Pu}$ including a gate to exclude side-feeding. . . . 162

6.1 Continuation of relaiive spot intensities for ${ }^{239} \mathrm{Pu} \ldots \ldots \ldots 163$

6.1 Continuation of relative spot intensities for ${ }^{239} \mathrm{Pu} \ldots \ldots \ldots$

6.1 Continuation of relative spot intensities for ${ }^{239} \mathrm{Pu} \ldots \ldots . .165$ 
6.2 Relative spot intensities for the probability of populating a given spin in ${ }^{238} \mathrm{Pu}$ including a gate to exclude side-feediag. . . . . 169

B.1 Comparison of FITEK results and raw integration methods . . 196 


\section{Experimental}

\section{Chapter 1}

\section{Introduction}

Nuclear physicists and chemists have long been concerned with the fundamental building blocks of our world. Because we have no tools to directly "see" these fundamental building blocks of nature, one has to resort to indirect observation. Nuclear reactions are performed with a variety of pr.bes to try to slucidate, from the resulting debris, something about the initial characteristics of the system. It is interesting to perturb a nucleus, study it under extreme conditions, and perhaps learn something about the underlying structure of the system. Two of the extreme conditions that are among the most studied today are high temperature and high pressure. Researchers collide nuclei at the highest possible energies, and use huge detector systems to study the resulting plethora of particles produced, and try to determine, for instance, the possible existence of the quark-gluon plasma. This thesis, however, is concerned with 
a third possible extremum, high angular momentum.

It is well known that spheroidally deformed nuclei exhibit rotational quantum states characterized by spin angular momentum states. For an even-even nucleus, the energy levels are given by the well-known equation $E_{\text {rot }}=\hbar^{2} I(I+1) / 2 \Im$, where $I$ is the spin of the nucleus, and $\Im$ is the momentof-inertia. Rotational states have been studied in the deformed rare earth region for the better part of 40 years by a variety of reactions $[1],[2],[3],\{4],\{5],[6],[7]$. Transfer reactions in the rare-earth region have yielded much information, not only on rotational states, but also on transfer mechanismis. One-nucleon transfer has been demonstrated to be a selective probe of single-particle and singlehole states [8] and two-nucleon transfer probes nucleon-nucleon correlations and pairing effects [9]. The earliest transfer reactions studied used light ion probes $((d, p)$ reactions $)[10],[11],\{12]$.

With the development of better heavy-ion accelerators, heavy-ion induced transfer reactions enjoyed growing success. Early work concentrated on light nucleus-nucleus systems $[13],[14]$. The development of even heavier beams allowed study of transfer reactions from rotationally excited states [15]. The largest difference in the physics of heavy-ion transfer reactions is the Coulomb excitation, caused by the large $Z$ of the projectile and target [16]. Since 
the Coulomb force is long range, and nucleon transfer occurs with highest probability near the distance of closest approach, transfer is likely to occur between rotationally excited states [17]. Few-nucleon transfer experiments combine the selectivity of studying one- and two-particle correlations with the selectivity of Coulomb excitation to add a new dimension to the study of nuclear structure [18]. Many experiments have been performed in the rareearth region (cf [19],,[20],\{21],[22], [23], [24],,[25],[26]), some in the actinides (cf $[20],\{27],[28])$ and some on spherical nuclei (cf [29],[30]).

Work by van Oertzen et al. [31] for Sn on Sn systems, and by Sapotta et al. [32] for $\mathrm{Ni}$ on ${ }^{208} \mathrm{~Pb}$ systems shows the expected slope dependence on neutron transfer as a function of distance of approach for spherical systems. Indeed, the one-neutron transfer with deformed systems [33] also shows normal behavior. It is two-neutron transfer with deformed systems that shows enhanced transfer at large distances. Enhancement factors have been measured for several systems [26].

Coulomb excitation has also been used in the regions between closed-shell spherical nuclei and well-deformed nuclei, or transitional regions. For instance, collective effects in the Se region [34] and in the $\mathrm{Pt}$ region [35] were investigated using Coulomb excitation. 
In the actinide region, however, most of the information on rotational states (high-spin states) has been from Coulomb excitation [36],[37],[38],[39],[40] and from $(\alpha, x n)$ reactions [41] and [42]. Transfer reactions have been extended into the actinide region [27]. This thesis will discuss high-spin states in the actinide region populated by heavy-ion inelastic excitation and neutron transfer reactions. Chapter 2 will discuss the experimental setups, Chapter 3 will discuss the results of the experiments and Chapter 6 shows the comparison between experimental rotational population patterns and theori: Chapters 4 and 5 are concerned with theories. 


\section{Chapter 2}

\section{Experimental Methods}

\subsection{General Description}

This section will describe the experimental setup for inelastic excitation reactions and neutron transfer reactions. A sample experimental configuration is shown in Figure 2.1. The exact details for each experiment will be given in later sections. Most experiments described in this work were done at Oak Ridge National Laboratory's (ORNL) Holifiedd Heavy Ion Research Facility (HHIRF) in the Spin Spectrometer. Some of the work was done at the SuperHILAC facility at Lawrence Berkeley Laboratory (LBL) in the Itigh Energy Resolution Array (HERA). All experiments had the following common features: (1) large number of Compton-suppressed intrinsic Ge detectors for detecting $\gamma$ rays with high resolution, and (2) position-sensitive parallel-plate avalanche 


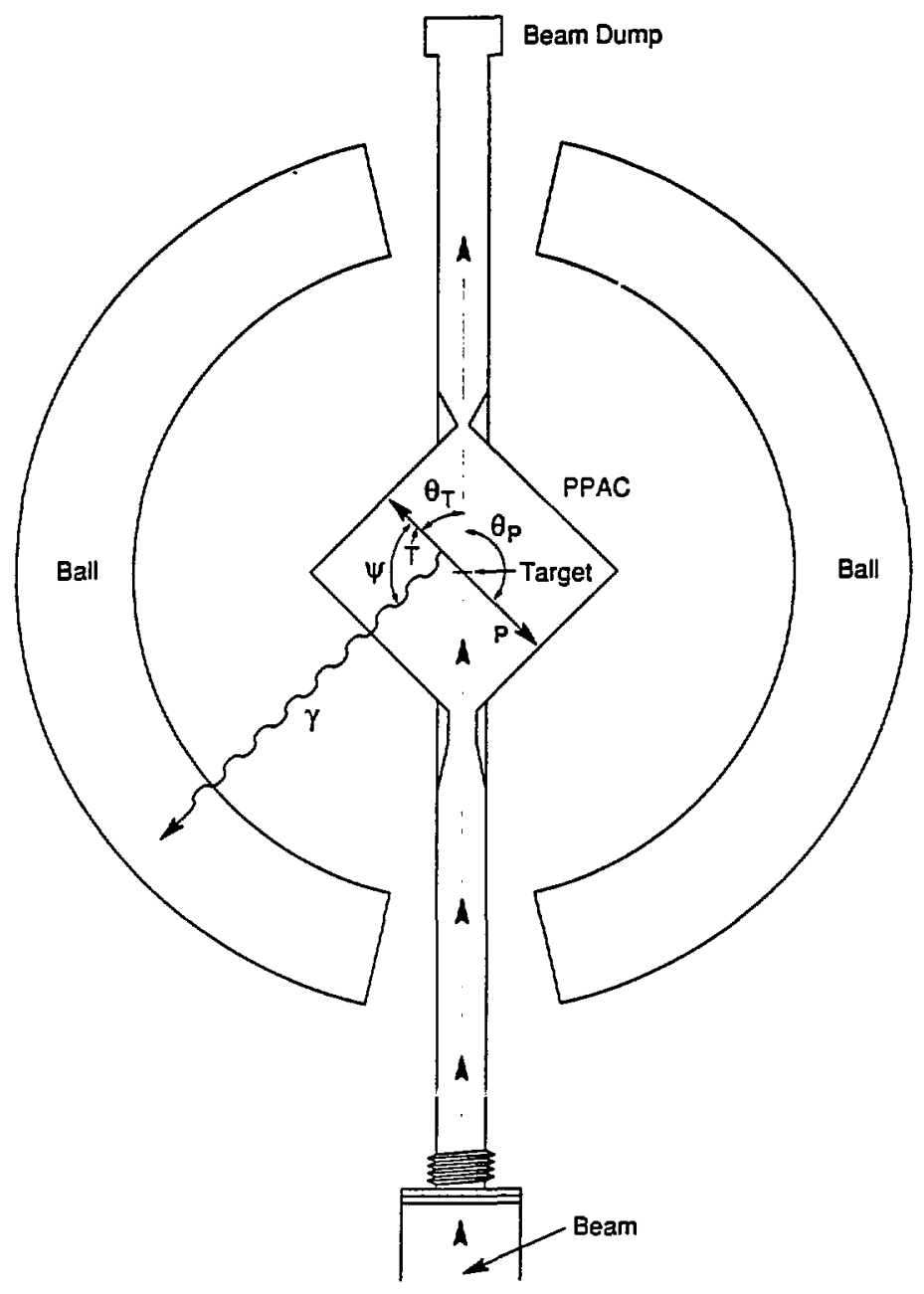

Figure 2.1: Schematic diagram of generic experimental configuration. 
counters (PPACs) for detecting projectile-like and target-like charged-particle reaction products. In addition, the ORNL experiments had a large number of $\mathrm{Nal}(\mathrm{Tl})$ scintillator detectors arranged in a ball covering $\sim 4 \pi$ solid angle. The study of heavy-ion transfer and scattering reactions poses severe problems for charged-particle spectroscopy, because, even though thin targets are used in the experiments, the multiple scattering and energy loss in the target degrades the energy resolution of the charged-particle detectors. The rotational energy levels of deformed nuclei are typically on the order of 100's of keV apart, requiring higher resolution methods. Thus, a particle-particle- $\gamma$ coincidence method is used, where the PPAC detectors give scattering angles and times-of-flight (TOF) of the target-like and projectile-like fragments in the lab (allowing a reconstruction of the kinematics of the reaction), and ihe Ge detectors give $\gamma$-ray energy information on the population of final product states. Because the $\gamma$-rays are emitted from a moving source (up to $\sim 10 \%$ the speed of light), the positional information from the PPACs allows for a Doppler correction of the $\gamma$-rays on an event-by-event basis to minimize the Doppler broadening and Doppler shift. In addition, the scattering angles also determine the distance of closest approach and impact parameters of the event. In the experiments at ORNL, the Spin Spectrometer NaI(TI) array provided 
total energy and multiplicity information for the event, and allowed for the separation of neutrons and $\gamma$-rays based on TOF. A more complete description of each of these elements of the experiments is given below.

A thin target, typically $\sim 400 \mu \mathrm{g} / \mathrm{cm}^{2}$ target material deposited on $\sim 100$ $\mu \mathrm{g} / \mathrm{cm}^{2} \mathrm{Ni}$ backing facing upstream, is used to minimize the disturbance of the kinematics. The PPACs are located approximately $10 \mathrm{~cm}$ from the target, and form the walls of the University of Rochester scattering chamber. PPACs are chosen because of the high counting rates inherent in these experiments. The angular coverages of the PPACs are given in the detailed discussion of each experiment. The PPACs contain flowing isobutane at 4 Torr and are separated from the evacuated target chamber by $\sim 50 \mu \mathrm{g} / \mathrm{cm}^{2}$ polypropylene film. Because of the polypropylene film, there is a low-energy threshold for charged particles to be detected in the PPAC of $\sim 50 \mathrm{keV}$. The PPACs outside walls are $\sim \frac{1}{16}$ " aluminum to minimize absorption of $\gamma$-rays. The cathodes are printed-circuit boards with $2^{\circ} \theta$ stripes. The $\theta$ stripes are interconnected with delay chips linking the stripes in a long chain, such that the difference in recorded times of a signal travelling to both ends of the cathode yields the $\theta$ position of the particle. The PPAC is calibrated in $\theta$ by placing an aluminum mask slotted at known angular positions in front of the polypropylene window 
of the counter that stops all scattered particles except for those at the known angles. A sample PPAC calibration spectrum is shown in Figure 2.2. The anodes are stretched aluminized polypropylene foils sectioned to yield $\phi$ information (resolution $\approx 15^{\circ}$ ) and spaced $3 \mathrm{~mm}$ from the cathodes. The anode is kept at $+400 \mathrm{~V}$ and provides a prompt timing signal for a charged particle traversing the detector. Because the main decay mode of the radioactive targets used in this study is $5-8 \mathrm{MeV} \alpha$-particles, the voltage is chosen to suppress the significant background source of $\alpha$-particles from the targets. A potential of $+500 \mathrm{~V}$ makes the PPACs sensitive to $\alpha$-particles. From the time difference between the particles and the reconstructed kinematics the mass of each particle can be determined. The mass resolution of a PPAC, $\sim 10-15$ amu, is insufficient for resolving particles differing by only one or two neutrons (as in the case of neutron transfer reactions), but sufficient for resolving an actinide-like particle from a lighter projectile-like particle. The outside wall of the PPAC stops all particles except neutrons.

The PPACs and scattering chamber are positioned inside a ball of Ge detectors (and at ORNL also $\mathrm{NaI}(\mathrm{Tl})$ detectors) for detecting $\gamma$-rays in coincidence with a projectile-like and target-like fragment. From fourteen to eightern $\sim 25 \%$ efficient intrinsic n-type Ge detectors plus either bismuth ger- 


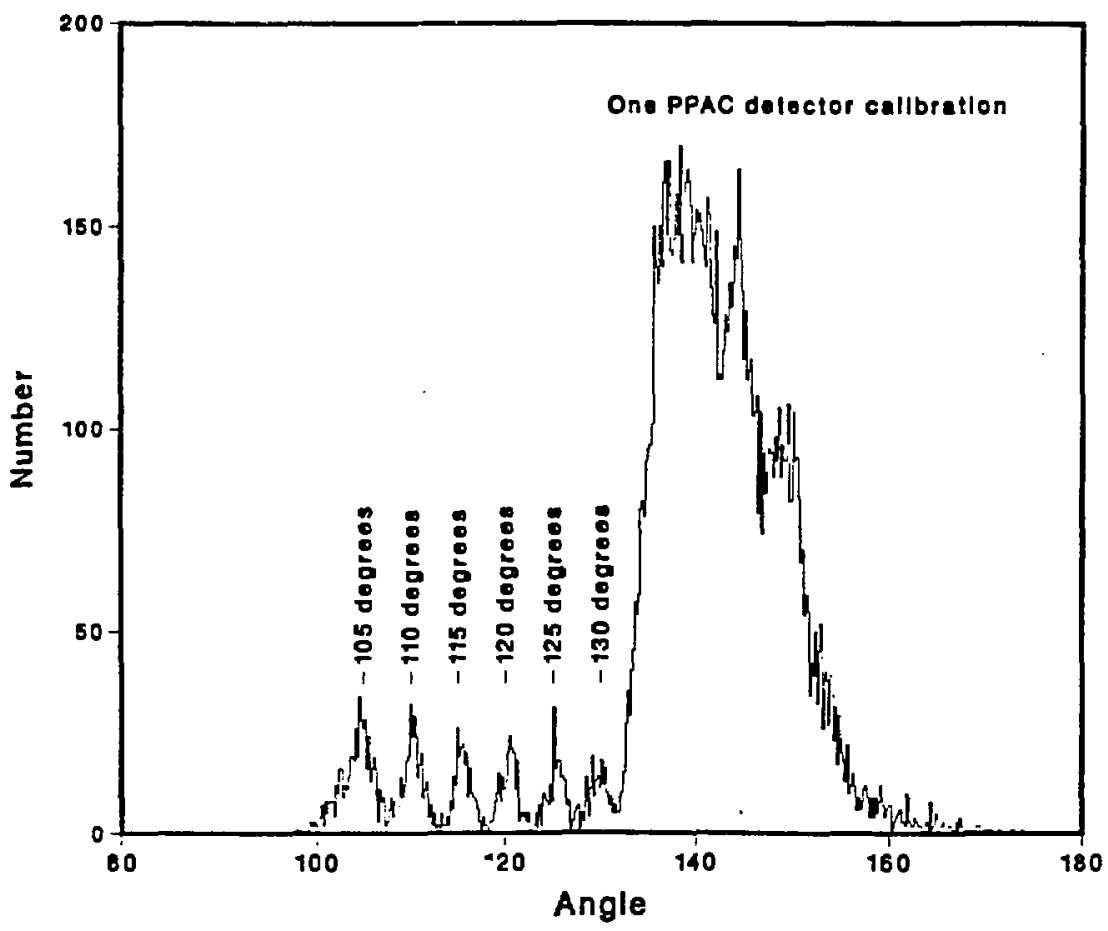

Figure 2.2: Calibration of particle scattering angles in a PPAC. 
manate (BGO) or $\mathrm{NaI}(\mathrm{Tl})$ anti-coincidence Compton shields were typically used. Any event depositing some energy in the Compton shield was rejected. This substantially reduces the background. These detectors were placed $\sim 20$ $\mathrm{cm}$ from the target. Absolute energy and efficiency calibrations were made with standard radioactive $\gamma$ - ray sources of ${ }^{182} \mathrm{Ta},{ }^{152} \mathrm{Eu},{ }^{60} \mathrm{Co}$ and a mixed source of ${ }^{125} \mathrm{Sb},{ }^{125} \mathrm{Te},{ }^{155} \mathrm{Eu}$, and ${ }^{154} \mathrm{Eu}$. The angular locations are given in the detailed descriptions of the experiments. The Ge detectors had a typical energy resolution of $1.7 \mathrm{keV}$ at $E_{\gamma}=1 \mathrm{MeV}$ and an efficiency of $\sim 1 \%$ at $200 \mathrm{keV} . \mathrm{Ta}\left(\sim 0.005^{\prime \prime}\right)$, Gu $\left(\sim 0.00 \mathrm{r}^{\prime \prime}\right)$ and $\mathrm{Sn}\left(\sim 0.010^{\prime \prime}\right)$ absorbers were placed in front of the Ge detectors to absorb the low-energy $\gamma$ - and $x$-rays (mostly background $\mathrm{x}$-ray radiation from the target). Thus, many low-energy rotational transitions like the $2^{+} \rightarrow 0^{+}$and $4^{+} \rightarrow 2^{+}{ }^{238} \mathrm{Pu}$ transitions were impossible to detect in our experimental setup because of the low-energy cutoff, and also because of the high conversion rates of these transitions. There was no attempt to detect conversion electrons, $e^{-}$.

For the experiments at ORNL, up to $5.5 \mathrm{NaI}(\mathrm{Tl})$ detectors in the Spin Spectrometer ball were also used in addition to Ge $\gamma$-ray detectors. These detectors were placed $\sim 18 \mathrm{~cm}$ from the target, and were used to detect $\gamma$-rays with high efficiency, and neutrons with lower efficiency. The slower neutrons 
were detected by $(\mathrm{a}, \gamma)$ reactions in the $\mathrm{NaI}(\mathrm{Tl})$, and can be separated from prompt $\gamma$-rays by TOF. Because of the high segmentation of the array, the ball yields information about the total energy of the reaction $\left(H=\sum_{N a I} E_{\uparrow}\right)$, total $\gamma$-ray multiplicity ( $K=$ number of $\mathrm{NaI}(\mathrm{Tl})$ fired), and total neutron multiplicity $\left(K_{n}\right)$ for an event. The $H, K$ matrix can be transformed to $(E, M)$ (total de-excitation energy, multiplicity $\rightarrow$ spin) through a procedure of "unfolding" with a ball respense function. The ball response function was constructed with standard radioactive $\gamma$-ray sources of ${ }^{60} \mathrm{Co},{ }^{207} \mathrm{Bi},{ }^{88} \mathrm{Y},{ }^{75} \mathrm{Se}$ and ${ }^{232} \mathrm{Th}$, and corrects for $\mathrm{NaI}(\mathrm{Tl})$ efficiency, double hits in the detectors, and $\gamma$-rays "lost" through spaces between the adjacent detectors. Jääskeläinen et. al. [43] characterized the response function for this particular detector setup. Figure 17 in reference [43] shows the multiplicity projections for the system for an experimental test with a given number of $1 \mathrm{MeV} \gamma$-rays. For $K=30$, the distribution is a Gaussian centered about a detected multiplicity of $30 \gamma$-rays; however, for $K=40$, the distribution is centered about a detected fold of $35 \gamma$-rays. As a rule of thumb then, for the Spin Spectrometer, as long as the total event multiplicity is less than or equal to half the number of $\mathrm{NaI}(\mathrm{Tl})$ detectors used in the experiment, the number of double hits in detectors approximately cancels the $\gamma$-rays that go undetected between detectors. However, if the average 
multiplicity is larger than half the number of $\mathrm{NaI}(\mathrm{Tl})$ detectors, corrections for double hits in the detectors become severe $(\sim 20-25 \%)$. Figure 18 in reference [43] shows the detected total energy for a given number of $1 \mathrm{MeV}$ $\gamma$-rays. For $20 \gamma$-says, the $E_{\text {tot }} \approx 20 \mathrm{MeV}$, however, for $30 \gamma$-rays the energy is only $\sim 25 \mathrm{MeV}$. Thus, the total energy detected deviates from the actual total energy deposited in the ball by about $20 \%$ when half the detectors fire in the ball. The response function for the Spin Spectrometer is discussed in reference [43].

\subsection{1 ${ }^{58} \mathrm{Ni}+{ }^{235} \mathrm{U}$ Reaction at ORNL}

This experiment was carried out using $325-\mathrm{MeV}{ }^{58} \mathrm{Ni}$ ions bombarding a $300 \mu \mathrm{g} / \mathrm{cm}^{2}{ }^{235} \mathrm{U}$ target with $110 \mu \mathrm{g} / \mathrm{cm}^{2} \mathrm{Ni}$ backing in the Spin Spectrometer at ORNL, which consisted oi $5.5 \mathrm{NaI}(\mathrm{Tl})$ and $14 \mathrm{Ge}$ detectors. Figure 2.3 shows a schematic diagram of the experimental configuration. The target composition is given in Table 2.1. Surrounding the target were four PPACs, covering lab angles of $8^{\circ}$ to $78^{\circ}$ and $104^{\circ}$ to $162^{\circ}$ in the 0 direction, and $-35^{\circ}$ to $35^{\circ}$ and $145^{\circ}$ to $215^{\circ}$ in the $\phi$ direction; the $+z$-axis is the beam direction, $\theta$ is the angle measured from the z-axis, and $\phi$ is the azimuthal angle measured 


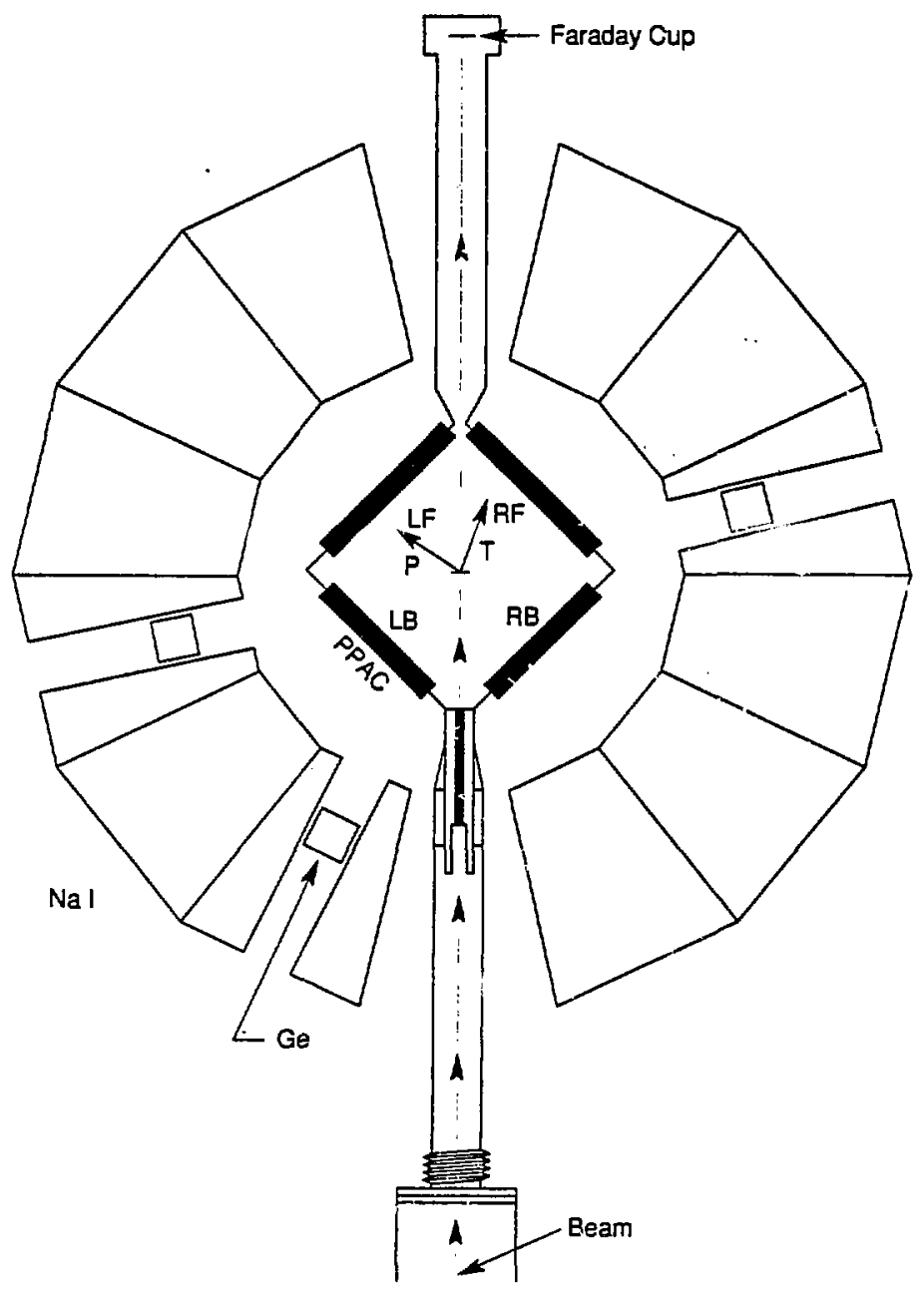

Figure 2.3: Diagram of ${ }^{58} \mathrm{Ni}+{ }^{235} \mathrm{U}$ experimental setup. 
Table 2.1: Isotopic composition of target for ${ }^{58} \mathrm{Ni}+{ }^{235} \mathrm{U}$ experiment

\begin{tabular}{|c|c|}
\hline Isotope & Percentage \\
\hline${ }^{235} \mathrm{U}$ & $99.85 \%$ \\
${ }^{234} \mathrm{U}$ & $0.034 \%$ \\
\hline
\end{tabular}

from the axis perpendicular to the horizontal plane in the lab. The PPACs are arranged as the sides of a cube and form the walls of the target chamber. Both the projectile-like and the target-like particles are detected. The lab angles of the Ge and $\mathrm{NaI}(\mathrm{Tl})$ detectors are given in Tables 2.2 and 2.3, respectively. The principle reaction studied was the one-neutron pickup reaction ${ }^{235} \mathrm{U}\left({ }^{58} \mathrm{Ni},{ }^{59} \mathrm{Ni}\right){ }^{234} \mathrm{U}\left(Q_{\text {react }}=3.73 \mathrm{MeV}, V_{c}=323.4 \mathrm{MeV}\right.$, where $V_{c}$ is the Coulomb barrier height).

Table 2.2: Lab detector positions for ${ }^{58} \mathrm{Ni}+{ }^{235} \mathrm{U}$ experiment

\begin{tabular}{|c|c|c|c|c|c|c|c|c|}
\hline \multicolumn{10}{|c|}{ Ge detector positions (angles in lab) } \\
\hline No. & $\theta$ & $\phi$ & No. & $\theta$ & $\phi$ & No. & $\theta$ & $\phi$ \\
\hline 1 & $155.6^{\circ}$ & $-4.9^{\circ}$ & 2 & $116.6^{\circ}$ & $18.0^{\circ}$ & 3 & $63.4^{\circ}$ & $-18.0^{\circ}$ \\
4 & $63.4^{\circ}$ & $126.0^{\circ}$ & 5 & $116.6^{\circ}$ & $-54.0^{\circ}$ & 6 & $24.4^{\circ}$ & $4.9^{\circ}$ \\
7 & $116.6^{\circ}$ & $-126.0^{\circ}$ & 8 & $116.6^{\circ}$ & $162.0^{\circ}$ & 9 & $138.4^{\circ}$ & $176.0^{\circ}$ \\
10 & $63.4^{\circ}$ & $-162.0^{\circ}$ & 11 & $41.0^{\circ}$ & $-176.0^{\circ}$ & 12 & $130.5^{\circ}$ & $-6.3^{\circ}$ \\
13 & $63.4^{\circ}$ & $54.0^{\circ}$ & 14 & $21.4^{\circ}$ & $148.9^{\circ}$ & & & \\
\hline
\end{tabular}


Table 2.3: Lab detector positions for ${ }^{58} \mathrm{Ni}+{ }^{235} \mathrm{U}$ experiment

\begin{tabular}{|c|c|c|c|c|c|c|c|c|}
\hline \multicolumn{10}{|c|}{ NaI(T) detector positions (angles in lab) } \\
\hline No. & $\theta$ & $\phi$ & No. & $\theta$ & $\phi$ & No. & $\theta$ & $\phi$ \\
\hline 2 & $24.4^{\circ}$ & $76.5^{\circ}$ & 4 & $24.4^{\circ}$ & $-67.1^{\circ}$ & 5 & $24.4^{\circ}$ & $-139.1^{\circ}$ \\
7 & $63.4^{\circ}$ & $-90.0^{\circ}$ & 8 & $87.3^{\circ}$ & $-95.4^{\circ}$ & 9 & $67.9^{\circ}$ & $-116.4^{\circ}$ \\
10 & $41.6^{\circ}$ & $-104.0^{\circ}$ & 11 & $49.5^{\circ}$ & $-65.7^{\circ}$ & 12 & $77.5^{\circ}$ & $-68.7^{\circ}$ \\
14 & $87.3^{\circ}$ & $-23.4^{\circ}$ & 15 & $67.9^{\circ}$ & $-44.4^{\circ}$ & 16 & $41.6^{\circ}$ & $-32.0^{\circ}$ \\
17 & $49.5^{\circ}$ & $6.3^{\circ}$ & 18 & $77.5^{\circ}$ & $3.3^{\circ}$ & 20 & $87.3^{\circ}$ & $48.6^{\circ}$ \\
21 & $67.9^{\circ}$ & $27.6^{\circ}$ & 22 & $41.6^{\circ}$ & $40.0^{\circ}$ & 23 & $49.5^{\circ}$ & $78.3^{\circ}$ \\
24 & $77.5^{\circ}$ & $75.3^{\circ}$ & 26 & $87.3^{\circ}$ & $120.6^{\circ}$ & 27 & $67.9^{\circ}$ & $99.6^{\circ}$ \\
28 & $41.6^{\circ}$ & $112.0^{\circ}$ & 29 & $49.5^{\circ}$ & $150.3^{\circ}$ & 30 & $77.5^{\circ}$ & $147.3^{\circ}$ \\
32 & $87.3^{\circ}$ & $-167.4^{\circ}$ & 33 & $67.9^{\circ}$ & $171.6^{\circ}$ & 35 & $49.5^{\circ}$ & $-137.7^{\circ}$ \\
36 & $77.5^{\circ}$ & $-140.7^{\circ}$ & 38 & $155.6^{\circ}$ & $-76.9^{\circ}$ & 40 & $155.6^{\circ}$ & $67.1^{\circ}$ \\
41 & $155.6^{\circ}$ & $139.1^{\circ}$ & 42 & $155.6^{\circ}$ & $-148.9^{\circ}$ & 43 & $116.6^{\circ}$ & $90.0^{\circ}$ \\
44 & $92.7^{\circ}$ & $95.4^{\circ}$ & 45 & $112.1^{\circ}$ & $116.4^{\circ}$ & 46 & $138.4^{\circ}$ & $104.0^{\circ}$ \\
47 & $130.5^{\circ}$ & $65.7^{\circ}$ & 48 & $102.5^{\circ}$ & $68.7^{\circ}$ & 50 & $92.7^{\circ}$ & $23.4^{\circ}$ \\
51 & $112.1^{\circ}$ & $44.4^{\circ}$ & 52 & $138.4^{\circ}$ & $32.0^{\circ}$ & 53 & $130.5^{\circ}$ & $-6.3^{\circ}$ \\
54 & $102.5^{\circ}$ & $-3.3^{\circ}$ & 56 & $92.7^{\circ}$ & $-48.6^{\circ}$ & 57 & $112.1^{\circ}$ & $-27.6^{\circ}$ \\
58 & $138.4^{\circ}$ & $-40.0^{\circ}$ & 59 & $130.5^{\circ}$ & $-78.3^{\circ}$ & 60 & $102.5^{\circ}$ & $-75.3^{\circ}$ \\
62 & $92.7^{\circ}$ & $-120.6^{\circ}$ & 63 & $112.1^{\circ}$ & $-99.6^{\circ}$ & 64 & $138.4^{\circ}$ & $-112.0^{\circ}$ \\
65 & $130.5^{\circ}$ & $-150.3^{\circ}$ & 66 & $102.5^{\circ}$ & $-147.3^{\circ}$ & 68 & $92.7^{\circ}$ & $167.4^{\circ}$ \\
69 & $112.1^{\circ}$ & $-171.6^{\circ}$ & 71 & $130.5^{\circ}$ & $137.7^{\circ}$ & 72 & $102.5^{\circ}$ & $140.7^{\circ}$ \\
\hline
\end{tabular}




\subsection{2 ${ }^{206} \mathrm{~Pb}+{ }^{233} \mathrm{U}$ Reaction at LBL}

This experiment was carried out using $1566-\mathrm{MeV}{ }^{206} \mathrm{~Pb}$ ions $(1200-\mathrm{MeV}$ at the center of the target) from the SuperHILAC bombarding a $430 \mu \mathrm{g} / \mathrm{cm}^{2}{ }^{233} \mathrm{U}$ target with a $2.1 \mathrm{mg} / \mathrm{cm}^{2}$ Be backing in HERA at LBL, which consisted of fourteen Ge detectors. Figure 2.4 gives a schematic diagram of the experimental configuration. The target composition is given in Table 2.4, and target construction is discussed in detail in Appendix A. Surrounding the target were

Table 2.4: Isotopic composition of target for ${ }^{206} \mathrm{~Pb}+{ }^{233} \mathrm{U}$ experiment

\begin{tabular}{|c|c|}
\hline Isotope & Percentage \\
\hline${ }^{233} \mathrm{U}$ & $99.8 \%$ \\
${ }^{232} \mathrm{U}$ & $<0.0038 \%$ \\
\hline
\end{tabular}

six PPACs covering lab angles of $15^{\circ}$ io $75^{\circ}$ in the $\theta$ direction, and $-25^{\circ}$ to $25^{\circ}, 35^{\circ}$ to $85^{\circ}, 95^{\circ}$ to $145^{\circ}, 155^{\circ}$ to $205^{\circ}, 215^{\circ}$ to $265^{\circ}$ and $275^{\circ}$ to $325^{\circ}$ in the $\phi$ direction. The PPACs are arranged as the sides of a pyramid with its apex on the beam axis, and both projectile-like and target-like particles are detected. The lab angles of Ge detectors are given in Table 2.5. The principle reaction studied was the inelastic excitation reaction ${ }^{233} \mathrm{U}\left({ }^{206} \mathrm{~Pb},{ }^{206} \mathrm{~Pb}^{\prime}\right){ }^{233} \mathrm{U}^{\prime}$ $\left(V_{c}=1220 \mathrm{MeV}\right)$ 


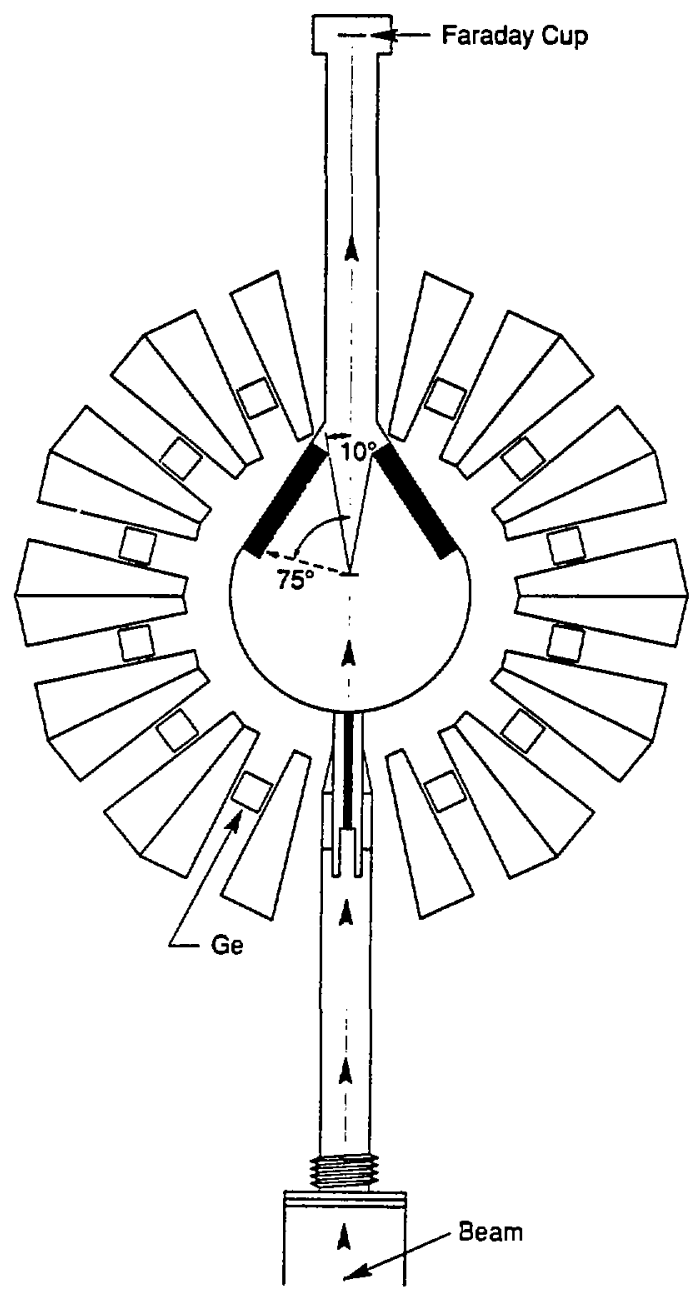

Figure 2.4: Diagram of ${ }^{206} \mathrm{~Pb}+{ }^{233} \mathrm{U}$ experimental setup. 
Table 2.5: Lab detector positions for ${ }^{206} \mathrm{~Pb}+{ }^{233} \mathrm{U}$ experiment

\begin{tabular}{|c|c|c|c|c|c|c|c|c|}
\hline \multicolumn{10}{c|}{ Ge detector positions (angles in lab) } \\
\hline No. & $\theta$ & $\phi$ & No. & $\theta$ & $\phi$ & No. & $\theta$ & $\phi$ \\
\hline 1 & $41.4^{\circ}$ & $0.0^{\circ}$ & 2 & $20.5^{\circ}$ & $-55.4^{\circ}$ & 3 & $21.1^{\circ}$ & $54.6^{\circ}$ \\
4 & $159.6^{\circ}$ & $0.0^{\circ}$ & 5 & $138.6^{\circ}$ & $37.7^{\circ}$ & 6 & $138.6^{\circ}$ & $-37.3^{\circ}$ \\
7 & $180.0^{\circ}$ & $90.0^{\circ}$ & 8 & $159.6^{\circ}$ & $180.0^{\circ}$ & 9 & $138.6^{\circ}$ & $127.3^{\circ}$ \\
10 & $180.0^{\circ}$ & $270.0^{\circ}$ & 11 & $48.3^{\circ}$ & $180.0^{\circ}$ & 12 & $75.0^{\circ}$ & $207.6^{\circ}$ \\
13 & $140.0^{\circ}$ & $218.3^{\circ}$ & 14 & $75.0^{\circ}$ & $117.6^{\circ}$ & & & \\
\hline
\end{tabular}




\subsection{3 ${ }^{58} \mathrm{Ni}+{ }^{233} \mathrm{U}$ Reaction at UOR}

This counting experiment was carried out using $249-\mathrm{MeV}^{58} \mathrm{Ni}$ ions bombarding a $430 \mu \mathrm{g} / \mathrm{cm}^{2}{ }^{233} \mathrm{U}$ target with $135 \mu \mathrm{g} / \mathrm{cm}^{2} \mathrm{Ni}$ and $4.0 \mathrm{mg} / \mathrm{cm}^{2} \mathrm{Cu}$ backing at the University of Rochester (UOR) Nuclear Structure Research Laboratory's Tandem Accelerator. The target was viewed by one BGO Compton-suppressed Ge detector located at $\theta=0^{\circ}$, two Ge detectors located at $\theta= \pm 90^{\circ}$, and a Si particle detector covering $135^{\circ}$ to $170^{\circ}$ in the $\theta$ direction. Backscattered $\mathrm{Ni}$ ions were detected in the $\mathrm{Si}$ detector, and the ${ }^{233} \mathrm{U}$ was allowed to recoil through the target backing, emerging with approximately $30 \mathrm{MeV}$ (a loss of about $120 \mathrm{MeV}$ in the backing). Doppler shifts at $0^{\circ}$ were decreased due to this slowing of recoils in the target backing, and the Doppler broadening of the lines was small in this experiment. The principle reaction studied was the Coulomb excitation reaction ${ }^{233} \mathrm{U}\left({ }^{58} \mathrm{Ni}^{58}{ }^{58} \mathrm{Ni}^{\prime}\right)^{233} \mathrm{U}^{\prime}$.

\subsection{4 ${ }^{90} \mathrm{Zr}+{ }^{239} \mathrm{Pu}$ Reaction at ORNL}

This experiment was carried out using $500-\mathrm{MeV}{ }^{90} \mathrm{Zr}$ ions bombarding a $356.3 \mu \mathrm{g} / \mathrm{cm}^{2}{ }^{239} \mathrm{Pu}$ target with a $170 \mu \mathrm{g} / \mathrm{cm}^{2}$ Ni backing in the Spin Spectrometer at ORNL, which consisted of $52 \mathrm{NaI}(\mathrm{Tl})$ and 18 Ge detectors. Fig- 
ure 2.5 gives a schematic diagram of the experimental configuration. The target composition is given in Table 2.6 , and the target construction is discussed in Appendix A. Surrounding the target were two PPACs covering lab

Table 2.6: Isotopic composition of target for ${ }^{90} \mathrm{Zr}+{ }^{239} \mathrm{Pu}$ experiment

\begin{tabular}{|c|c|}
\hline Isotope & Percentage \\
\hline${ }^{239} \mathrm{Pu}$ & $93.826 \%$ \\
${ }^{238} \mathrm{Pu}$ & $0.012 \%$ \\
${ }^{240} \mathrm{Pu}$ & $5.913 \%$ \\
${ }^{241} \mathrm{Pu}$ & $0.222 \%$ \\
${ }^{242} \mathrm{Pu}$ & $0.027 \%$ \\
${ }^{241} \mathrm{Am}$ & $<1 \%$ \\
${ }^{23} \mathrm{U}$ & $\ll 1 \%$ \\
\hline
\end{tabular}

angles of $104^{\circ}$ to $162^{\circ}$ in the $\theta$ direction, and $-35^{\circ}$ to $35^{\circ}$ and $145^{\circ}$ to $215^{\circ}$ in the $\phi$ direction. The PPACs were arranged as the upstream two sides of a cube and formed the wails of the scattering chamber. Only backscattered $\mathrm{Zr}$ ions were detected in this experiment; the $\mathrm{Pu}$ scattering angle was inferred from the scattering kinematics. The lab angles of the $\mathrm{Ge}$ and $\mathrm{NaI}(\mathrm{Tl})$ detectors are given in Tables 2.7 and 2.8 , respectively. The principle reaction studied was the one-neutron pickup reaction ${ }^{239} \mathrm{Pu}\left({ }^{90} \mathrm{Zr},{ }^{91} \mathrm{Zr}\right){ }^{238} \mathrm{Pu}\left(Q_{\text {react }}=1.58 \mathrm{MeV}\right.$, $\left.V_{c}=493.9 \mathrm{McV}\right)$, but the inelastic excitation reaction ${ }^{239} \mathrm{Pu}\left({ }^{90} \mathrm{Zr},{ }^{90} \mathrm{Zr}^{\prime}\right){ }^{239} \mathrm{Pu}^{\prime}$ was also studied in this setup. 


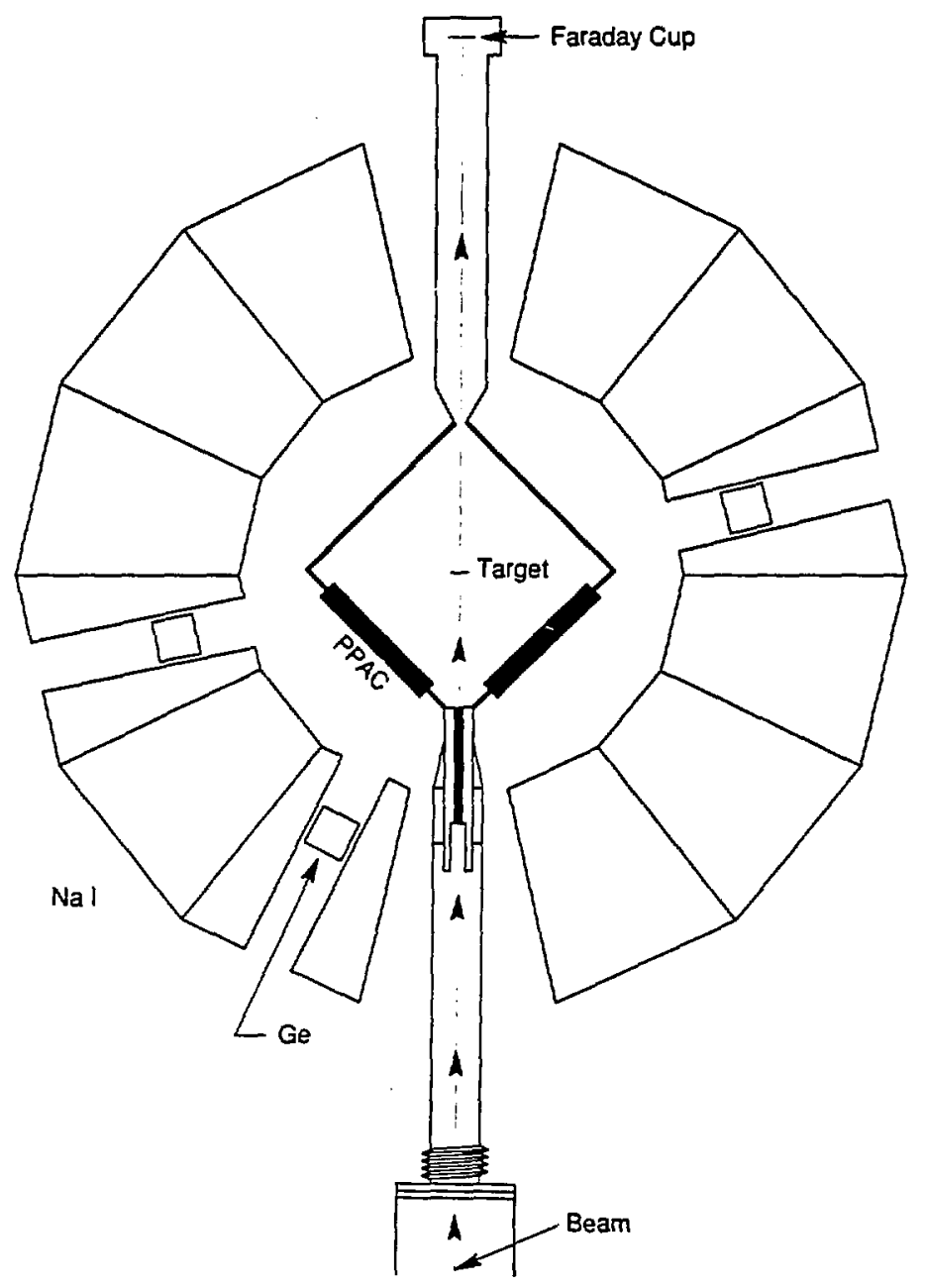

Figure 2.5: Diagram of ${ }^{90} \mathrm{Zr}+{ }^{239} \mathrm{Pu}$ experimental setup. 
Table 2.7: Lab detector positions for ${ }^{90} \mathrm{Zr}+{ }^{239} \mathrm{Pu}$ experiment

\begin{tabular}{|c|c|c|c|c|c|c|c|c|}
\hline \multicolumn{7}{c|}{ Ge detector positions (angles in lab) } \\
\hline No. & $\theta$ & $\phi$ & No. & $\theta$ & $\phi$ & No. & $\theta$ & $\phi$ \\
\hline 1 & $63.4^{\circ}$ & $-162.0^{\circ}$ & 2 & $92.7^{\circ}$ & $23.4^{\circ}$ & 3 & $155.6^{\circ}$ & $-148.9^{\circ}$ \\
4 & $116.6^{\circ}$ & $162.0^{\circ}$ & 5 & $63.4^{\circ}$ & $-18.0^{\circ}$ & 6 & $155.6^{\circ}$ & $139.1^{\circ}$ \\
7 & $116.6^{\circ}$ & $-126.0^{\circ}$ & 8 & $63.4^{\circ}$ & $126.0^{\circ}$ & 9 & $24.4^{\circ}$ & $148.9^{\circ}$ \\
10 & $24.4^{\circ}$ & $-139.1^{\circ}$ & 11 & $63.4^{\circ}$ & $54.0^{\circ}$ & 12 & $116.6^{\circ}$ & $18.0^{\circ}$ \\
13 & $24.4^{\circ}$ & $-67.1^{\circ}$ & 14 & $116.6^{\circ}$ & $-54.0^{\circ}$ & 15 & $155.6^{\circ}$ & $-4.9^{\circ}$ \\
16 & $138.4^{\circ}$ & $176.0^{\circ}$ & 17 & $49.5^{\circ}$ & $6.3^{\circ}$ & 18 & $138.4^{\circ}$ & $32.0^{\circ}$ \\
\hline
\end{tabular}

Table 2.8: Lab detector positions for ${ }^{90} \mathrm{Zr}+{ }^{239} \mathrm{Pu}$ experiment

\begin{tabular}{|c|c|c|c|c|c|c|c|c|}
\hline \multicolumn{10}{c|}{ NaI(Tl) detector positions (angles in lab) } \\
\hline No. & $\theta$ & $\phi$ & No. & $\theta$ & $\phi$ & No. & $\theta$ & $\phi$ \\
\hline 2 & $24.4^{\circ}$ & $76.8^{\circ}$ & 3 & $24.4^{\circ}$ & $4.9^{\circ}$ & 7 & $63.4^{\circ}$ & $-90.0^{\circ}$ \\
8 & $87.3^{\circ}$ & $-95.4^{\circ}$ & 9 & $67.9^{\circ}$ & $-116.4^{\circ}$ & 10 & $41.6^{\circ}$ & $-104.0^{\circ}$ \\
11 & $49.5^{\circ}$ & $-65.7^{\circ}$ & 12 & $77.5^{\circ}$ & $-68.7^{\circ}$ & 14 & $87.3^{\circ}$ & $-23.4^{\circ}$ \\
15 & $67.9^{\circ}$ & $-44.4^{\circ}$ & 16 & $41.6^{\circ}$ & $-32.0^{\circ}$ & 18 & $77.5^{\circ}$ & $3.3^{\circ}$ \\
20 & $87.3^{\circ}$ & $48.6^{\circ}$ & 21 & $67.9^{\circ}$ & $27.6^{\circ}$ & 22 & $41.6^{\circ}$ & $40.0^{\circ}$ \\
23 & $49.5^{\circ}$ & $78.3^{\circ}$ & 24 & $77.5^{\circ}$ & $75.3^{\circ}$ & 26 & $87.3^{\circ}$ & $120.6^{\circ}$ \\
27 & $67.9^{\circ}$ & $99.6^{\circ}$ & 28 & $41.6^{\circ}$ & $112.0^{\circ}$ & 29 & $49.5^{\circ}$ & $150.3^{\circ}$ \\
30 & $77.5^{\circ}$ & $147.3^{\circ}$ & 32 & $87.3^{\circ}$ & $-167.4^{\circ}$ & 33 & $67.9^{\circ}$ & $171.6^{\circ}$ \\
34 & $41.6^{\circ}$ & $-176.0^{\circ}$ & 35 & $49.5^{\circ}$ & $-137.7^{\circ}$ & 36 & $77.5^{\circ}$ & $-140.7^{\circ}$ \\
38 & $15.5 .6^{\circ}$ & $-76.9^{\circ}$ & 40 & $155.6^{\circ}$ & $67.1^{\circ}$ & 43 & $116.6^{\circ}$ & $90.0^{\circ}$ \\
44 & $92.7^{\circ}$ & $95.4^{\circ}$ & 45 & $112.1^{\circ}$ & $116.4^{\circ}$ & 46 & $138.4^{\circ}$ & $104.0^{\circ}$ \\
47 & $130.5^{\circ}$ & $65.7^{\circ}$ & 48 & $102.5^{\circ}$ & $68.7^{\circ}$ & 51 & $112.1^{\circ}$ & $44.4^{\circ}$ \\
53 & $130.5^{\circ}$ & $-6.3^{\circ}$ & 54 & $102.5^{\circ}$ & $-3.3^{\circ}$ & 56 & $92.7^{\circ}$ & $-48.6^{\circ}$ \\
57 & $112.1^{\circ}$ & $-27.6^{\circ}$ & 58 & $138.4^{\circ}$ & $-40.0^{\circ}$ & 59 & $130.5^{\circ}$ & $-78.3^{\circ}$ \\
60 & $102.5^{\circ}$ & $-75.3^{\circ}$ & 62 & $92.7^{\circ}$ & $-120.6^{\circ}$ & 63 & $112.1^{\circ}$ & $-99.6^{\circ}$ \\
64 & $135.4^{\circ}$ & $-112.0^{\circ}$ & 65 & $130.5^{\circ}$ & $-150.3^{\circ}$ & 66 & $102.5^{\circ}$ & $-147.3^{\circ}$ \\
68 & $92.7^{\circ}$ & $167.4^{\circ}$ & 69 & $112.1^{\circ}$ & $-171.6^{\circ}$ & 71 & $130.5^{\circ}$ & $137.7^{\circ}$ \\
72 & $102.5^{\circ}$ & $140.7^{\circ}$ & & & & & & \\
\hline
\end{tabular}




\subsection{Data Analysis Techniques}

An event written on raw data tapes consisted of uncalibrated energies and time signals from the PPACs, $\mathrm{NaI}(\mathrm{Tl})$ and Ge detectors. These tapes were then scanned on an event-by-event basis and rewritten on prescan tapes which recorded the new and calibrated parameters. An event on the prescan tape consisted of scattering angles $\theta, \phi$ of the particles in the lab, timing information between the left and right PPACs, Doppler-corrected and energycalibrated $\mathrm{Ge}$ and $\mathrm{NaI}(\mathrm{Tl}) E_{\gamma}$ spectra; and new parameters calculated from the observables, mass observed in a given PPAC, neutron multiplicity $\left(K_{n}\right)$, number of Ge detectors firing $\left(K_{G e}\right)$, sum of $\mathrm{NaI}(\mathrm{Tl})$ energies $(H)$ and the number of NaI(Tl) detectors firing $(K)$. Doppler corrections to the Ge spectra were made for both the projectile-like and target-like fragments. The Doppler corrected $\gamma$-ray energy for the target-like fragment is given by:

$$
E_{\gamma}^{\prime}=\frac{E_{\gamma}}{1+\beta(D O P P)},
$$

where $E_{\gamma}$ is the raw, uncorrected $\gamma$-ray energy, and $\beta$ and $D O P P$ are defined in the foilowing manner:

$$
\beta=\frac{p}{A_{T}} \frac{1}{931.5016},
$$


where $A_{T}$ is the mass number of the target-like fragment, and the momentum is defined in the usual way

$$
p=\frac{p_{\text {tot }} \sin \theta_{T}}{v_{\text {trans }}}
$$

$p_{\text {tot }}$ is the total initial momentum of the projectile, or

$$
p_{\text {tot }}=\sqrt{2 E_{\text {beam }} A_{P}}
$$

In the ${ }^{90} \mathrm{Zr}+{ }^{239} \mathrm{Pu}$ case, $p_{\text {tot }}=9156.1 \mathrm{MeV} / \mathrm{c}$. The lab scattering angle of the target-like fragment is $\theta_{T}$, and the transverse velocity, $v_{t r a n s}$, is given by:

$$
v_{\text {trans }}=\sin \theta_{P}+\theta_{T},
$$

where $\theta_{P}$ is the lab scattering angle of the projectile-like fragment. The Doppler shift is given by:

$$
D O P P=\hat{x}_{G e} \cdot \hat{p}_{x}+\hat{y}_{G e} \cdot \hat{p}_{y}+\hat{z}_{G e} \cdot \hat{p}_{z},
$$

where $\hat{x}_{G e}, \hat{y}_{G e}$, and $\hat{z}_{G e}$ are unit vectors denoting the $\mathrm{x}, \mathrm{y}$ and $\mathrm{z}$ direction in the lab of the Ge detectors (the target being defined as the origin with the beam direction the $+z$-axis), and

$$
\begin{aligned}
& \dot{p}_{x}=\sin \theta_{T} \cos \phi_{T} \\
& \dot{p}_{y}=\sin \theta_{T} \sin \phi_{T} \\
& \dot{p}_{z}=\cos \theta_{T} .
\end{aligned}
$$


Similar equations exist for the Doppler correction of the projectile-like fragment. The Doppler correction is made on an event-by-event basis for each Ge detector individually. Doppler corrections for the NaI(Tl) detectors were made in some cases, however, in general the Doppler corrections for a $\mathrm{NaI}(\mathrm{Tl})$ detector are negligible compared to the resolution of the scintillator (Doppler shifts are on the order of $2-5 \mathrm{keV}, \mathrm{NaI}(\mathrm{Tl})$ resolution $\sim 50 \mathrm{keV}$ ).

For the reactions studied in which both the target-like recoil fragment and the projectile-like scattered fragment were detected, the beam energy, entrance channel masses, TOFs, angles of the trajectories, and timing difference between the particles are enough to calculate the mass of a particle observed in a PPAC, assuming two-body kinematics. A mass spectrum for the ${ }^{206} \mathrm{~Pb}+$ ${ }^{233} \mathrm{U}$ reaction is shown in Figure 2.6. As Figure 2.6 shows, the mass resolution of the PPACs is insufficient to resolve particles $A$ and $A+1$, but a Pb-like and U-like separation can clearly be made. As mentioned previously, $\theta$ calibrations were determined by placing an $\mathrm{Al}$ mask with known slits over the PPACs and running the reaction for a short time. Figure 2.2 shows a sample calibration spectrum for the ${ }^{90} \mathrm{Zr}+{ }^{239} \mathrm{Pu}$ reaction. A linear least-squares fit for the angular calibration is used.

Figure 2.7 shows a ${ }^{152}$ Eu calibration spectrum for one Ge detector. Peak 




Figure 2.6: Mass spectrum from one $\mathrm{PPAC}$ for the ${ }^{206} \mathrm{~Pb}+{ }^{233} \mathrm{U}$ reaction. 




Figure 2.7: ${ }^{152} \mathrm{Eu}$ calibration spectrum for a Ge detector. The dei etor resolution and background suppression should be noted. 
positions and intensities were obtained using the Gaussian least- squares fitting program FITEK. The FITEK program was developed by Dr. Wolfgang Stöft at Lawrence Livermore Nutional Laboratory (LLNL) and is discussed further in Appendix B. Using $\sim 20$ standard reference $\gamma$-rays from radioactive sources, linear least-squares energy calibrations are obtained for all Ge detectors. In addition, all Ge timing spectra are corrected. Figure 2.8 shows a typical uncorrected Ge $E_{\gamma}$ spectrum, a Doppler corrected $E_{\gamma}$ spectrum, and a Ge timing spectrum for the ${ }^{90} \mathrm{Zr}+{ }^{239} \mathrm{Pu}$ reaction. Timing resolution is $8 \mathrm{~ns}$ full-width-half-maximum (FWHM).

Doppler corrections and energy calibrations were performed again for experiments with $\mathrm{Nal}(\mathrm{Tl})$ detectors, ie., at the ORNL Spin Spectrometer. In addition, for detectors with sufficient timing, neutrons and $\boldsymbol{\gamma}$-rays are resolved from each other via the TOF of the neutrons. Figure 2.9 shows an example of a timing spectrum for a $\mathrm{Nal}(\mathrm{Tl})$ detector in the ${ }^{90} \mathrm{Zr}+{ }^{239} \mathrm{Pu}$ reaction for events depositing $2-16 \mathrm{MeV}$ in the detector. The $\mathrm{NaI}(\mathrm{Tl})$ timing is energy dependent, with a neutron-gamma separation impossible to make below 200 $\mathrm{keV}$. The time separation between the $\gamma$-rays and the neutrons is $5 \mathrm{~ns}$, which is about the same FWHM NaI(T) timing resolution $(4-5 \mathrm{~ns})$. Only detected $\gamma$-ray energies are added to the total energy spectrum. In addition, for most 

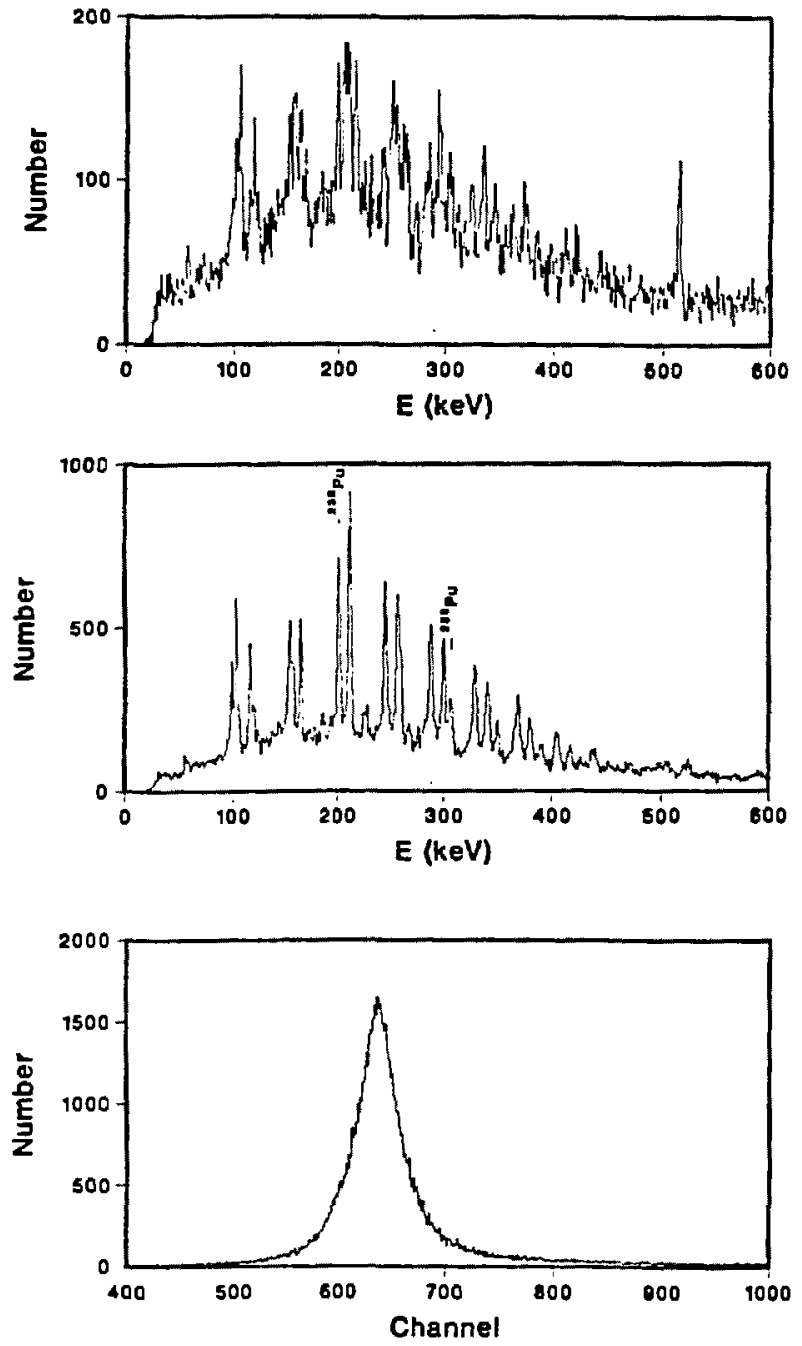

Figure 2.8: The top figure is an uncorrected Ge $E_{\gamma}$ spectrum. The middle figure is the same Ge $E_{\gamma}$ spectrum with Doppler corrections, and the bottom figure is the Ge timing spectrum. 


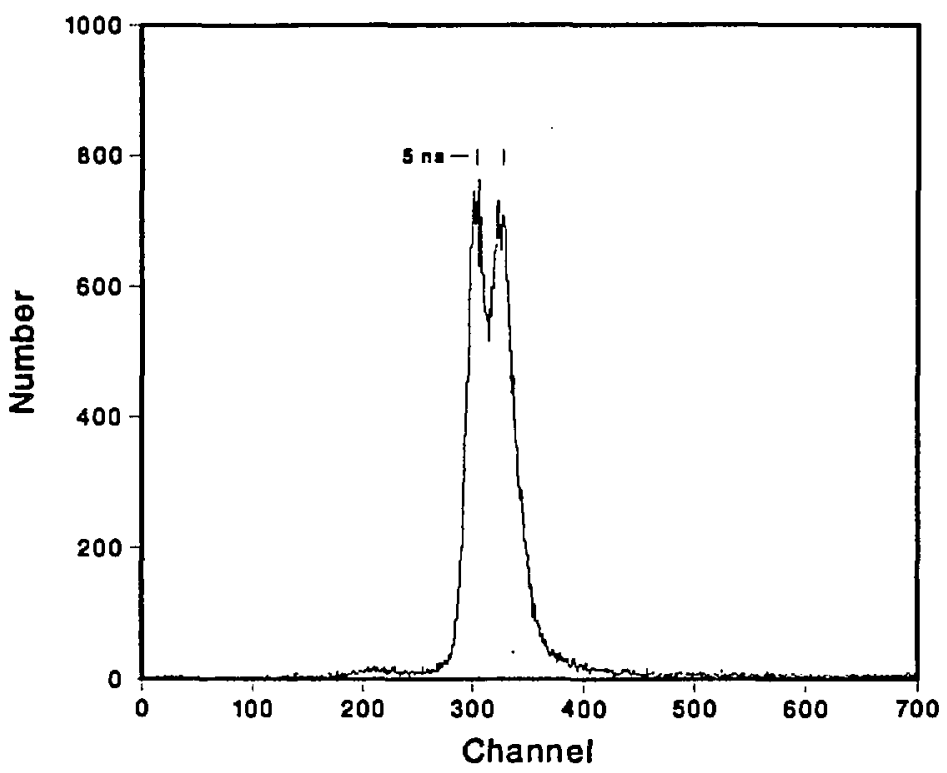

Figure 2.9: $\mathrm{NaI}(\mathrm{Tl})$ timing spectrum showing the neutron- $\gamma-$ ray separation for the ${ }^{96} \mathrm{Zr}+{ }^{239} \mathrm{Pu}$ reaction for events depositing $2-16 \mathrm{MeV}$ in the detector. 
cases in the remainder of this thesis, except where otherwise noted, a $K_{n}=0$ gate is used when analyzing events any further. This insures that the reaction being studied is not contaminated with neutron evaporation or fission events. As expected, $\mathrm{NaI}(\mathrm{Tl})$ detectors with high rates of neutron events were the ones located at forward angles in the lab, as both fission and neutron evaporation would occur from sources moving downstream.

Any parameter on the prescan tapes can then be histogrammed for further analysis. In addition, a variety of gates (different reaction conditions) or cuts could be applied to the data. Parameters could be histogrammed in a two-dimensional matrix versus other parameters to study correlations between different quantities. The results of these analyses are presented in Chapter 3. 


\section{Chapter 3}

\section{Results and Discussion}

\section{$3.1{ }^{58} \mathrm{Ni}+{ }^{235} \mathrm{U}$ Reaction}

The LBL/UOR/UTK/ORNL collaboration performed this experiment in the actinides as an exploratory effort to test the effectiveness of the heavy-ion "cold" one-neutron transfer technique, as a high-spin spectroscopic tool. The "cold" transfer mechanism is characterized by population of states in the product nucleus on or near the Y'rast line. The actinide region, as mentioned earlier, is a difficult region in which to obtain data, as stable targets do not exist. In addition to the natural radioactivity of the targets, fission is expected to be a dominant exit channel for any reaction above the fission barrier $(\sim 5 \mathrm{MeV}$ in the light actinides). Consequently, our first effort was more along the lines of proving the uscfulness of the method, and a longer-lived ${ }^{235} \mathrm{U}$ target was used. 
Figure 3.1 shows the Ge spectra; Figure 3.1a is the total $\gamma$-ray spectrum summed over all $14 \mathrm{Ge}$ detectors with appropriate Doppler corrections, Figure $3.1 \mathrm{~b}$ is a $\gamma-\gamma$ coincidence turo-fold $\gamma$-ray spectrum gated on the previously known $8^{+} \rightarrow 6^{+}, 10^{+} \rightarrow 8^{+}$and $12^{+} \rightarrow 10^{+}$transitions in ${ }^{234} \mathrm{U}$, and Figure 3.1c is the $\gamma$-ray spectrum gated on the $(H, K)$ spectrum from the $\mathrm{NaI}(\mathrm{Tl})$ detectors $(H>2.9 \mathrm{MeV}, K>4)$. Figure 3.1 a shows a $\gamma$-ray spectrum dominated by ${ }^{235} \mathrm{U}$ inelastic transitions and ${ }^{234} \mathrm{U}$ neutron transfer transitions. No appreciable fission competition was observed. The differential cross-section for the oneneutron pickup reaction was about $20 \mathrm{mb} / \mathrm{sr}$ at the grazing angle $\left(\sim 130^{\circ}\right.$ in the lab).

Figure $3.1 \mathrm{~b}$ clearly separates the ground rotational band of ${ }^{234} \mathrm{U}$ from inelastic contamination, although the spectrum suffers from the traditional lack of statistics associated with the $\gamma-\gamma$ coincidence methods. Nevertheless, the $22^{+} \rightarrow 20^{+}{ }^{234} \mathrm{U}$ transition can clearly be identified. Figure 3.1c, gated on $\left(H, K^{\prime}\right)$ in a region where one-neutron transfer should dominate, clearly shows the $24^{+} \rightarrow 22^{+}{ }^{234} \mathrm{U}$ transition. With some imagination, there is a candidate for the $26^{+} \rightarrow 24^{+}{ }^{23+} \mathrm{U}$ transition. It should be noted, that this is $6-8$ units of angular momentum higher than the highest spins populated by Coulomb excitation with ${ }^{58} \mathrm{Ni}$ ions in this region [36]. Also, the statistics are much 


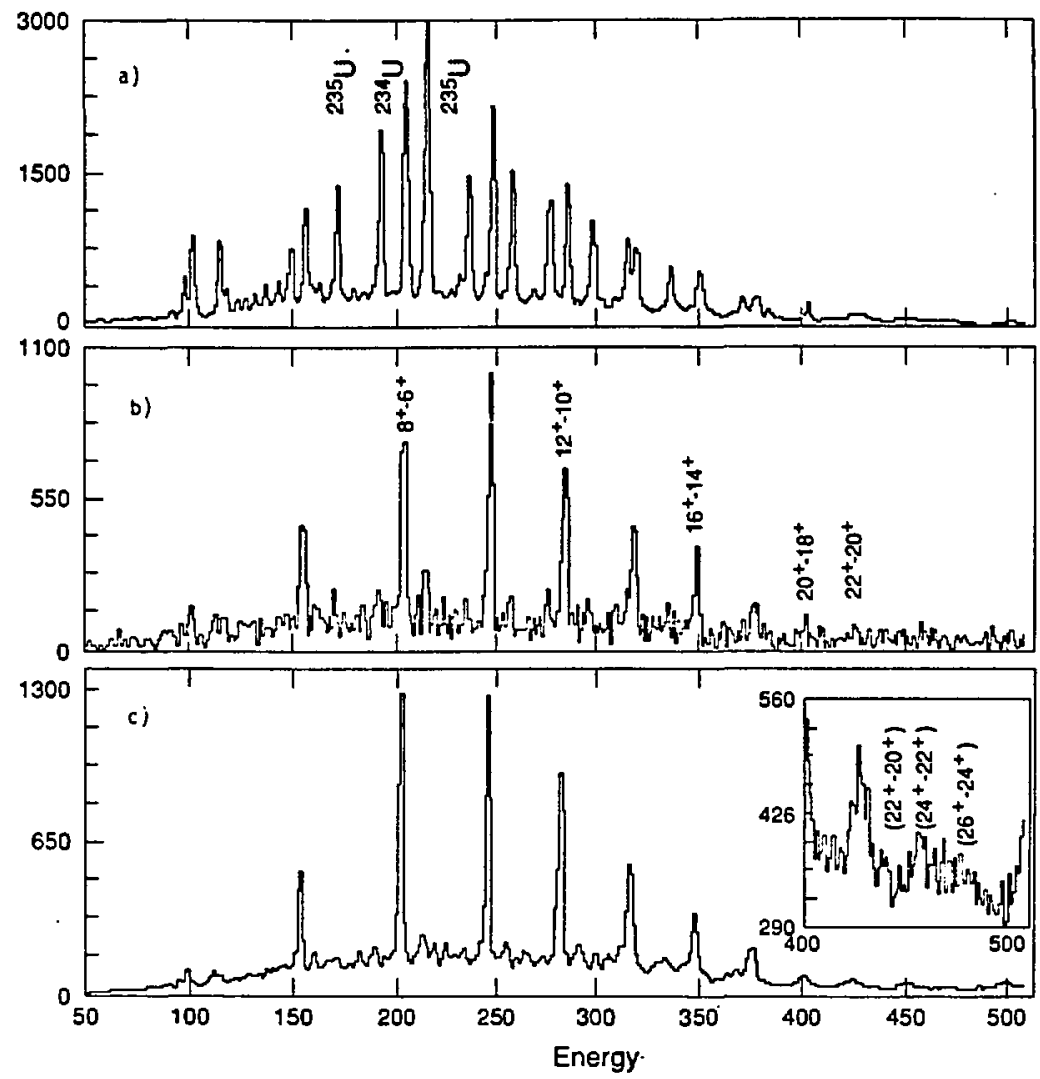

Figure 3.1: Ge $\gamma$-ray spectrum for the ${ }^{58} \mathrm{Ni}+{ }^{235} \mathrm{U}$ reaction; a) is the total $\gamma$ ray spectrum of all 14 Ge detectors summed together; $b$ ) is a $\gamma-\gamma$ coincidence spectrum gated on three previously known ${ }^{234} \mathrm{U}$ transitions; and $\left.c\right)$ is the $\gamma$-ray spectrum gated on the $\left(H, K^{*}\right)$ spectrum. Transitions from the $24^{+}\left(26^{+}\right)$level can clearly be identified in the inset to $c$ ). 
better in this method of using the $\mathrm{NaI}(\mathrm{Tl})$ ball to separate the transfer exit channel from the inelastic exit channel.

Figure 3.2 shows the total $E$, multiplicity, $M$, spectrum for the one-neutron transfer. It was produced by summing again on gates of the $8^{+} \rightarrow 6^{+}$, $10^{+} \rightarrow 8^{+}$, and $12^{+} \rightarrow 10^{+}$transitions in ${ }^{234} \mathrm{U}$ in the Ge detectors. The dashed line indicates the approximate Yrast line for ${ }^{234} \mathrm{U}$ assuming no ${ }^{58} \mathrm{Ni}$ excitation, and a conversion scale to spin, $I / \hbar$, is provided. Two maxima in the contour plot are observed, the lower one lying approximately on the Yrast line at $E=0.5 \mathrm{MeV}, M=1.5$. Inelastic events from target impurities are less than $10 \%$ of this peak. The upper maximum is centered at $E=3 \mathrm{MeV}, M=3.5$ and is $80 \%$ of the strength of the lower maximum. The upper maximum is $\sim 2 \mathrm{MeV}$ above the Y'rast line, still relatively low in excitation energy.

Figure 3.2 clcarly indicates that the reaction is "cold" and populates states on or within $2 \mathrm{MeV}$ of the Y'rast line. They can be understood through a mechanism in which kinematic and binding energy favors population near the Yrast line, and structure effects favor population of the rotationally aligned states. The lower bump is interpreted as population of states following the pickup of the odd neutron (the unpaired $j_{\frac{15}{2}}$ neutron), ie., the direct population of the ground-state band. The upper bump represents the population of states 


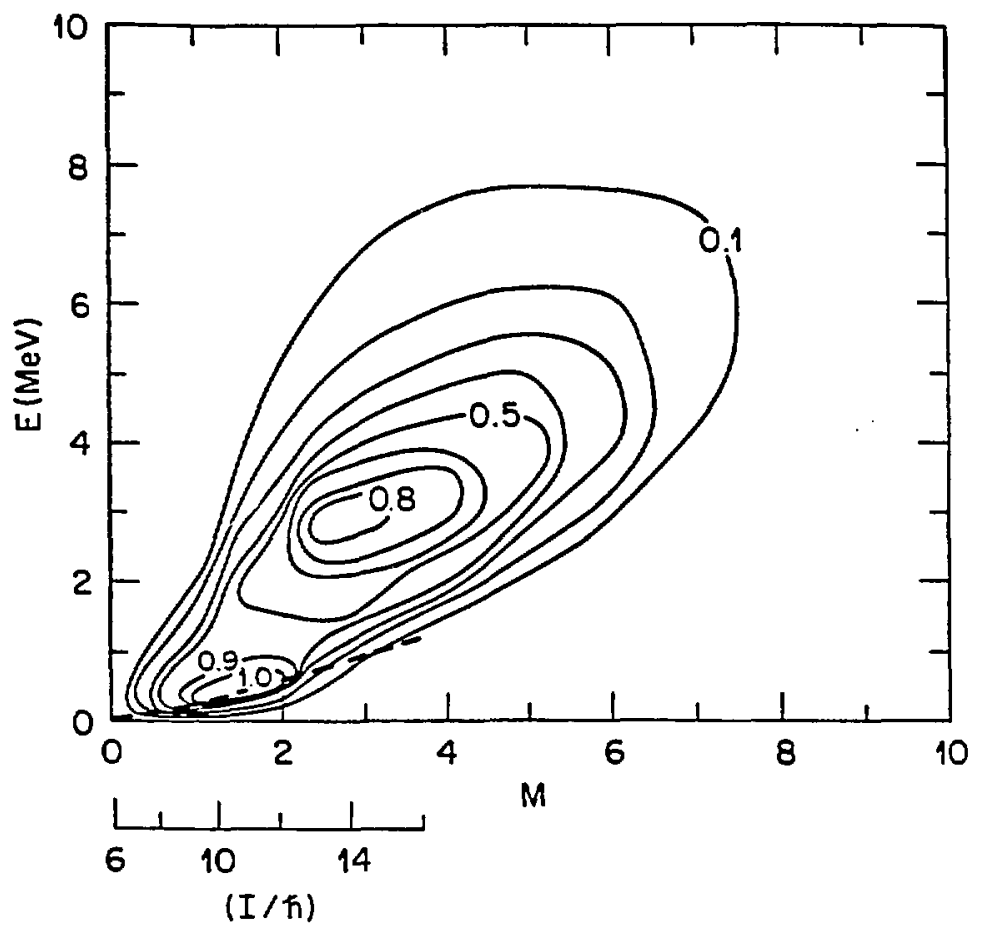

Figure 3.2: Total $E, .11$ plot for the ${ }^{58} \mathrm{Ni}+{ }^{235} \mathrm{U}$ reaction where the dashed line indicates the approximate Yrast line for ${ }^{234} \mathrm{U}$ assuming no excitation of the ${ }^{58} \mathrm{Ni}$. 
following the pickup of a paired neutron after the pair is broken leading to a two-quasiparticle state of higher excitation energy (hence the $\sim 2 \mathrm{MeV}$ above the Yrast line). The average alignment for each state populated in the twoquasiparticle region is about 6 , accounting for the multiplicity difference of about 3 between the upper and lower bumps in the $(H, K)$ spectrum if the de-excitation is by $E 2$ transitions. It should be pointed out that population of states with spin $\geq 26$ is most certainly occurring, but that a lack of statistics prevents identification of the discrete $\gamma$-rays.

The data presented here clearly establish this method as excellent for studying high-spin states in the actinides. The "cold" reaction mechanism minimizes fission competition. One-neutron transfer reactions populate higher spin states than does Coulomb excitation. Spins up to $24^{+}\left(26^{+}\right)$in ${ }^{234} \mathrm{U}$ are seen for the first time in this experiment. In Coulomb excitation experiments with $\mathrm{Pb}$ projectiles, states with spins $\sim 30 \hbar$ have been reached in the actinides $[40],[44],\{45],[46]$. This suggests that discrete states with spins higher than $30 \hbar$ could be populated in one-neutron transfer reactions using $\mathrm{Pb}$ projectiles. This directly led to the experiments described in the next section. 


\section{2 ${ }^{206} \mathrm{~Pb}+{ }^{233} \mathrm{U}$ and ${ }^{58} \mathrm{Ni}+{ }^{233} \mathrm{U}$ Reactions}

A total inelask.. excitation $\gamma$-ray spectrum (sum of 14 Ge detectors all appropriately Doppler corrected) from the SuperHILAC experiment is shown in Figure 3.3. Rotational transitions up to $\frac{49}{2}^{+} \rightarrow \frac{45}{2}^{+}$in ${ }^{233} \mathrm{U}$ are observed. In addition, the $8^{+} \rightarrow 6^{+}$transition in ${ }^{232} \mathrm{U}$ from the one-neutron transfer reaction ${ }^{233} \mathrm{U}\left({ }^{206} \mathrm{~Pb},{ }^{207} \mathrm{~Pb}\right){ }^{232} \mathrm{U}$ is observed. Insufficient statistics and contamination from ${ }^{233} \mathrm{U}$ lines precluded $\gamma-\gamma$ gating studies of other ${ }^{232} \mathrm{U}$ rotational transitions. Table 3.1 and Figure 3.4 contain a more complete presentation of both branches or signatures of the ground-state band transitions observed in ${ }^{233} \mathrm{U}$ from both experiments. Figure 3.4 shows the two signatures separately;

Table 3.1: Observed transitions in ${ }^{233} \mathrm{U}$

\begin{tabular}{|c|c|c|c|}
\hline Transition & Energy (keV) & Transition & Energy (keV) \\
\hline$\frac{11}{2}^{+} \rightarrow \frac{7}{2}^{+}$ & $113.0(2)$ & $\frac{13}{2}^{+} \rightarrow \frac{9}{2}^{+}$ & $137.6(2)$ \\
$\frac{15}{2}^{+} \rightarrow \frac{11}{2}^{+}$ & $160.1(2)$ & ${\frac{1 \frac{1}{7}^{+}}{2} \rightarrow \frac{13}{2}^{+}}^{+}$ & $181.6(2)$ \\
$\frac{19}{2}^{+} \rightarrow \frac{15}{2}^{+}$ & $203.1(2)$ & $\frac{21}{2}^{+} \rightarrow \frac{17}{2}^{+}$ & $223.6(2)$ \\
$\frac{23}{2}^{+} \rightarrow \frac{19}{2}^{+}$ & $243.0(2)$ & $\frac{25}{2}^{+} \rightarrow \frac{21}{2}^{+}$ & $262.5(2)$ \\
$\frac{27}{2}^{+} \rightarrow \frac{23}{2}^{+}$ & $281.9(2)$ & $\frac{29}{2}^{+} \rightarrow \frac{25}{2}^{+}$ & $299.3(2)$ \\
$\frac{31}{2}^{+} \rightarrow \frac{27}{2}^{+}$ & $316.7(2)$ & $\frac{33}{2}^{+} \rightarrow \frac{29}{2}^{+}$ & $334.1(4)$ \\
$\frac{35}{2}^{+} \rightarrow \frac{31}{2}^{+}$ & $349.5(4)$ & $\frac{37}{2}^{+} \rightarrow \frac{33}{2}^{+}$ & $366.9(4)$ \\
$\frac{39}{2}^{+} \rightarrow \frac{35}{2}^{+}$ & $381.2(4)$ & $\frac{41}{2}^{+} \rightarrow \frac{37}{2}^{+}$ & $397.6(4)$ \\
$\frac{43}{2}^{+} \rightarrow \frac{39}{2}^{+}$ & $409.8(4)$ & $\frac{45}{2}^{+} \rightarrow \frac{41}{2}^{+}$ & $423.2(4)$ \\
$\frac{17}{2}^{+} \rightarrow \frac{43}{2}^{+}$ & $436.5(4)$ & $\frac{49}{2}^{+} \rightarrow \frac{45}{2}^{+}$ & $418.7(4)$ \\
\hline
\end{tabular}




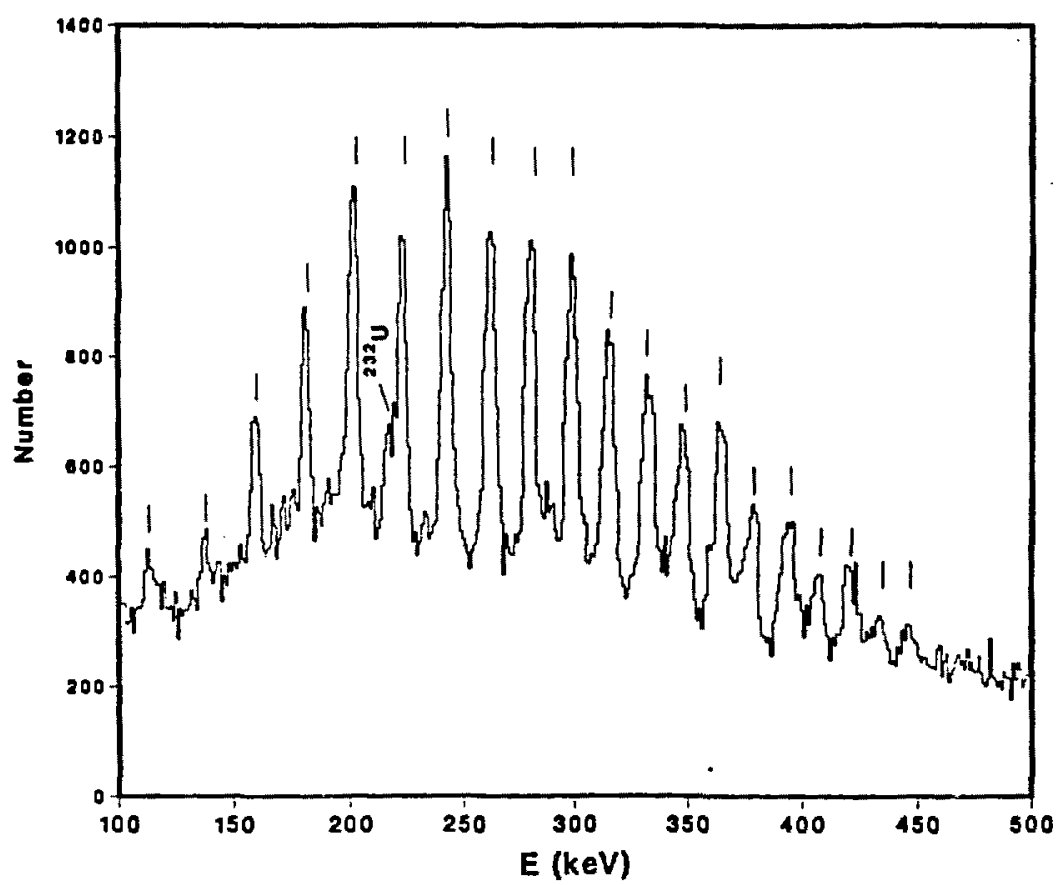

Figure 3.3: Total $\gamma$-ray spectrum of ${ }^{206} \mathrm{~Pb}\left({ }^{233} \mathrm{U}^{233} \mathrm{U}^{\prime}\right)^{206} \mathrm{~Pb}^{\prime}$ inelastic excitation reaction with beam energy of $1566 \mathrm{MeV}$. The spectrum is a sum of 14 Ge detectors, all appropriately Doppler corrected, and is gated on scattering angles of $45-70^{\circ}$ in the lab. The ${ }^{233} \mathrm{U}$ transitions are marked with dashes. Particle $\phi$ information was lost in some PPAC's resulting in broadening of lines. Note the transition at $217.4 \mathrm{keV}$ that is identified as the $8^{+} \rightarrow 6^{+}$transition in ${ }^{232} \mathrm{U}$, which is from the one-neutron transfer reaction. 




Figure 3.4: Level diagram for both signatures of the ground-state band in ${ }^{233} \mathrm{U}$. Arrows indicate the transitions observed in these experiments. The $\frac{9}{2}^{+} \rightarrow \frac{5}{2}^{+}$ and $\frac{17}{2}+\frac{15}{2}^{+}$transitions were masked by uranium $X$-rays. Errors in the last digit of the level energies are shown in parentheses (eg., 92.16(20) $=92.16 \pm$ $0.2 \mathrm{keV})$. ? he levels up to the $\frac{11}{2}^{+}$level are based on the Nuclear Data Tables [47], and ti:e remaining levels are obtained by adding transition energies. 
Recently, such methods have also been used by Dasso et al. [81] and [82] to calculate Diabolical pair transfer (SQUID effect). They do not include nuclear potential effects nor off-diagonal transfer matrix elements; however, they do include the S-band. Whether population favors the S-band or the ground band after the band crossing is solely dependent on the strength of the mixing matrix elements at the crossing point, something not well calculable by anyone. If the mixing is strong, the Coulomb excited population would tend to remain in the Yrast band, since $B(E 2)$ matrix elements between Yrast states exceed those connecting to the band above Yrast. However, if it is weak, population could go to both bands above the virtual crossing. Mixing matrix elements are difficult to calculate theoretically, and, while we have chosen for simplicity initially not to include the S-band in the calculations, this band is certainly straightforward to include. In addition, the mixing matrix elements could be taken from experiment. 
The transition energy is related to the cranking frequency by $\frac{E_{i}-E_{f}}{\Delta l}=\hbar \omega$. The moment-of-inertia has been calculated several ways; using the transition energy, $\Delta E=E_{i}-E_{f}$

$$
\frac{2 \Im^{(1)}}{\hbar^{2}}=\frac{I_{i}\left(I_{i}+1\right)-I_{f}\left(I_{f}+1\right)}{\Delta E}
$$

where $I_{i}$ and $I_{f}$ are the initial and final spin states, respectively; and using the first differen se of transition energies,

$$
\frac{2 \Im^{(2)}}{\hbar^{2}}=\frac{2 \Delta I}{\Delta(\hbar \omega)}
$$

where $\Delta(\hbar \omega)=\Delta\left(\frac{\Delta E}{2}\right)$. The moments-of-inertia, $\Im^{(1)}$ and $\Im^{(2)}$, are generally referred to as the "kinematic" and "dynamic" moments-of-inertia, respectively.

A brie? examination of the moment-of-inertia plots (Figure 3.5 and Figure 3.6) shows a steadily increasing moment-of-inertia, with nothing dramatic in the way of upbending or backbending. This change in the moment-of-inertia is probably a combination of alignment and a decrease in the pairing gap, $\Delta$, rather than a change in deformation. Recently, Helgesson and Hamamoto have explored the effects of triaxiality on the moments-of-inertia [49] in the rare earth region, and have shown smooth yet significant effects as a function of $\gamma_{d}$ in the region $-60^{\circ} \leq \gamma \leq 0^{\circ}$. For $N=90, A=152$ the moment-of-inertia $\Im_{x}$ 


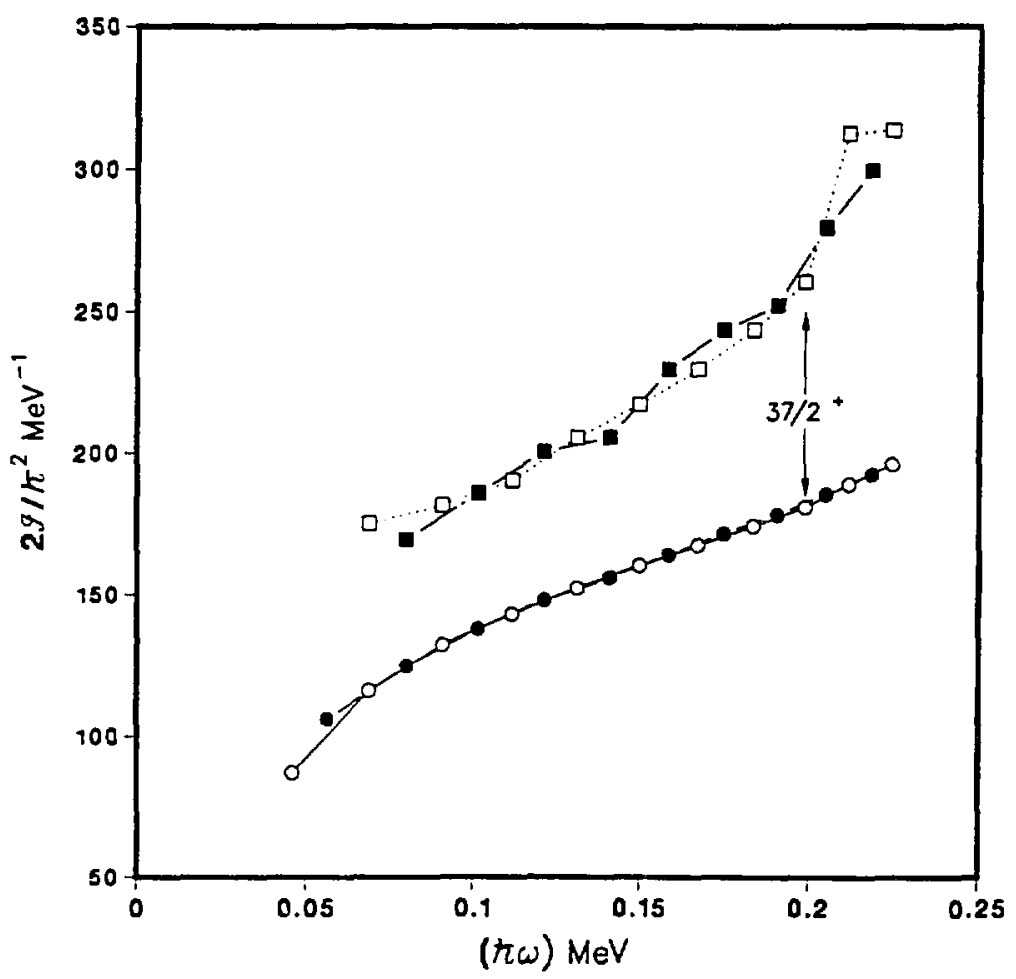

Figure 3.5: Plot of 3 vs. $\omega$ for ground-state band in ${ }^{233} \mathrm{U}$. The moment-ofinertia, $\Im$, was calculated using equations (3.1) $[0$ and $\bullet$, and (3.2) [open and sulid squares] in the text. The open symbols are for the $\frac{5}{2}^{+}$signature and the solid symbols are for the $\frac{7}{2}^{+}$signature. The errors in the moment-of-inertia are smaller than the plotted points. 


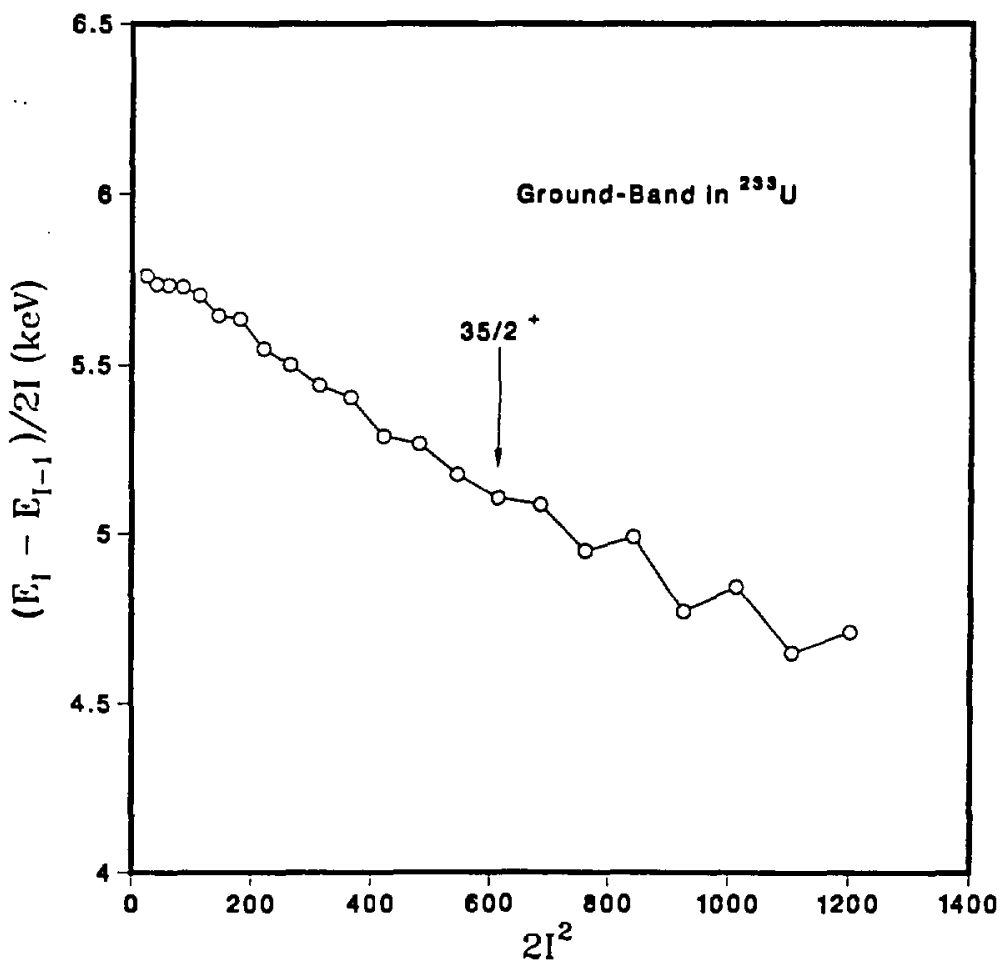

Figure 3.6: Plot of $\frac{E_{l}-E_{l-1}}{2 I}$, the inverse moment-of-inertia $\left(\frac{\hbar^{2}}{2 g^{(1)}}\right)$ for $\Delta I=1$ transitions, vs. $2 I^{2}$ showing the magnitude of the staggering term. 
decreases by a factor of two from $\gamma=0^{\circ}$ to $\gamma=-60^{\circ}$ (see their Figure 1). Thus, a small amount of triaxiality may be important in interpreting the change in the moment-of-inertia. In addition, the rotational level spacings appear to be very regular (see Figure 3.4), and the plot of the inverse moment-of-inertia $\left(h^{2} /\left(2 \Im^{(1)}\right)\right)$ for $\Delta I=1$ transitions vs. $2 J^{2}$, as shown in Figure 3.6, indicates the staggering term is small. However, on closer examination of Figure 3.6, it appears that the stagger reverses in sense part way up the rotational groundstate band (at about spin $\frac{35}{2}^{+}$). Additionally, in Figure 3.5 there is a change in slope of the $\Im^{(2)}$ plot in the same region $\left(\sim \frac{37}{2}^{+}\right)$. This spin is only about $\frac{2}{3}$ that of the predicted position of band crossings for neighboring even-even nuclej. These subtler features are perhaps indicating a soft band crossing or strong band mixing.

A single-particle level Nilsson calculation using P. Möller's code PEMOM [50] shows the ground-state configuration of the odd neutron to be $\frac{5}{2}$ [633] (standing for the usual asymptotic quantum numbers $K\left[N n_{x} \Lambda\right]$ ) derived from the $\nu 2 g \frac{9}{2}$ orbital mainly. A series of Cranking calculations using RSM [51] were then performed to study the neutron configuration as a function of spin. The Cranking model is well known and was not developed or modifie $I$ in this thesis. A more complete discussion of Cranking can be found elsewhere 
[52]. In this thesis the basis used is indicated in Table 3.2 with a core of 32 protons and 50 neutrons. The quadrupole and hexadecapole deformations, $\beta_{2}$

Table 3.2: Orbitals used as basis for Cranking model calculations

\begin{tabular}{|l|c|}
\hline$\pi=+$ & neutrons \\
\hline$\pi=-$ & $1 j \frac{11}{2}, 1 i \frac{13}{2}, 2 g \frac{7}{2}, 2 g \frac{9}{2}, 3 d \frac{3}{2}, 3 d \frac{5}{2}, 2 h \frac{11}{2}, 2 f \frac{5}{2}, 3 p \frac{1}{2}, 3 p \frac{3}{2}$ \\
\hline$\pi=+$ & $1 i \frac{11}{2}, 1 i \frac{13}{2}, 2 d \frac{3}{2}, 3 s \frac{1}{2}$ \\
\hline$\pi=-$ & $1 h \frac{9}{2}, 1 h \frac{11}{2}, 2 f \frac{5}{2}, 2 f \frac{7}{2}, 3 p \frac{1}{2}, 3 p \frac{3}{2}$ \\
\hline
\end{tabular}

and $\beta_{4}$, the pairing gap parameters, $\Delta_{n}$ and $\Delta_{p}$, and the chemical potentials, $\lambda_{n}$ and $\lambda_{p}$, were fixed at angular momentum zero, $\omega=0$, at the values of Kumar and Baranger [53]. The deformations and pairing parameters were kept fixed for all spins, but the chemical potential was allowed to vary. No number projection was done, because the number of valence particles is rather large compared to the number of core particles, $\lambda$ does not vary much, and the need to pull particles from the core is minimal. Thus the total number of valence particles was kept correct on average. For each angular momentum, 
the cranking frequency $\omega$ was determined by the usual cranking condition

$$
\langle J\rangle+\omega \Im_{c}=[I(I+1)]^{-\frac{1}{2}}
$$

where $\langle J\rangle$ is the angular momentum of the valence particles along the rotational axis and $\Im_{c} / \hbar^{2}=10 \mathrm{MeV}^{-1}$ is the moment-of-inertia of the core. Many calculations were performed to compare results when $\beta, \Delta, \lambda$, and $\Im_{c}$ values were varied. Figure 3.7 presents the results for the standard Kumar and Baranger parameters $\left(\beta_{2}=0.240, \beta_{4}=0.142, \Delta_{p}=0.900, \Delta_{n}=0.680\right)$ and for a case where $\beta_{2}=0.300$, as they reasonably well reproduced the first few energy level spacings of the ground rotational band. The fit at higher spins is not as satisfactory, perhaps because the deformation parameters were not allowed to vary as one goes up the band. The calculation with the increased $\beta_{2}$ tends to fit the lower spin region better, but again has the problem of too much alignment at higher spins. Other calculations were performed to try to fit the high-spin energy levels by increasing the deformation and pairing parameters and neglecting what happened at lower spins, but no satisfactory fit was obtained. The ground state configuration is mainly a mixture of $\frac{5}{2}[633](66 \%)$ and $\frac{5}{2}[622](20 \%)$ in agreement with the Nilsson calculation. However, at $I=\frac{49}{2}^{+}$, the configuration is mainly $\frac{1}{2}[651](41 \%)$, indicating alignment. Ta- 


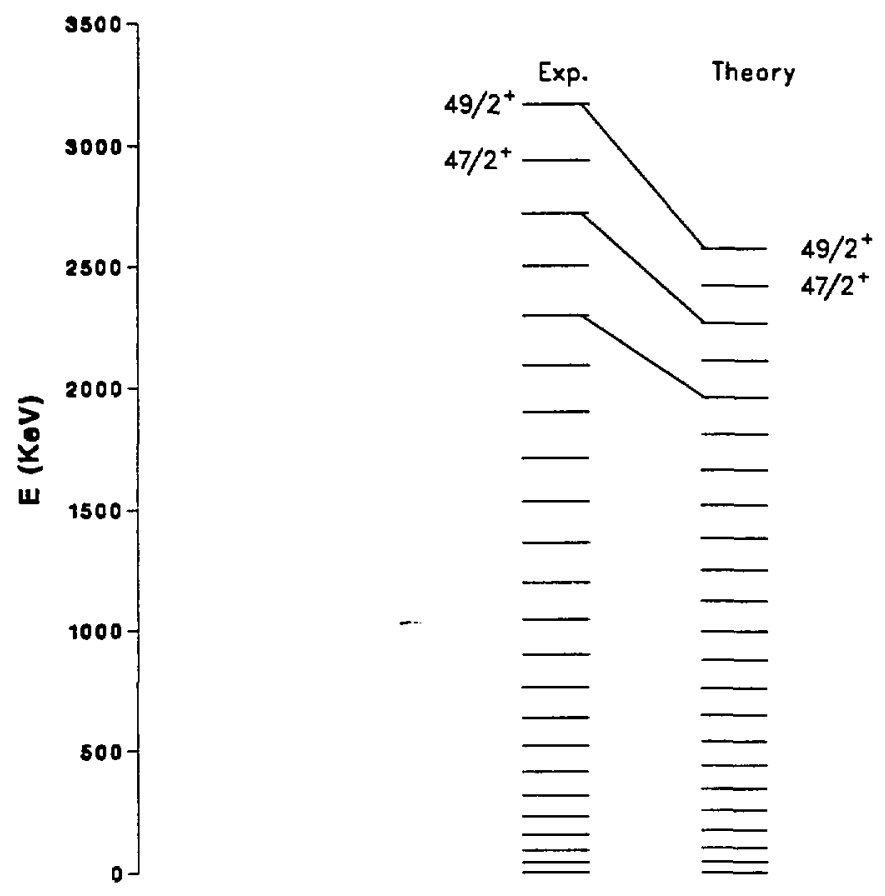

Figure 3.7: A comparison between experimental energy levels and theoretical energy levels for the ground rotational band in ${ }^{233} \mathrm{U}$. The theoretical energy levels are calculated using the cranking model described in the text and the standard Kumar and Baranger parameters. 
ble 3.3 shows the major portions of the wavefunctions (amplitude squared $>$ $16 \%$ ) as a function of spin. Alignment sets in for the theoretical calculations

Table 3.3: Main parts of the ground-state band wavefunction for ${ }^{233} \mathrm{U}$ in Cranking model

\begin{tabular}{|l|c|c|l|l|l|}
\hline$I^{\pi}$ & $K\left[N n_{2} \Lambda\right]$ & Prob. & $I^{\pi}$ & $K\left[N n_{2} \Lambda\right]$ & Prob. \\
\hline$\frac{5}{2}^{+}$ & $\frac{5}{2}[633]$ & 0.197 & $\frac{7}{2}^{+}$ & $\frac{5}{2}[633]$ & 0.185 \\
& $\frac{5}{2}[622]$ & 0.663 & & $\frac{5}{2}[622]$ & 0.613 \\
\hline$\frac{9}{2}^{+}$ & $\frac{5}{2}[633]$ & 0.166 & $\frac{11}{2}^{+}$ & $\frac{5}{2}[622]$ & 0.466 \\
& $\frac{5}{2}[622]$ & 0.543 & & & \\
\hline$\frac{13}{2}^{+}$ & $\frac{5}{2}[622]$ & 0.398 & $\frac{15}{2}^{+}$ & $\frac{5}{2}[622]$ & 0.345 \\
& & & & $\frac{1}{2}[651]$ & 0.195 \\
\hline$\frac{17}{2}^{+}$ & $\frac{5}{2}[622]$ & 0.301 & $\frac{19}{2}^{+}$ & $\frac{5}{2}[622]$ & 0.269 \\
& $\frac{1}{2}[651]$ & 0.230 & & $\frac{1}{2}[651]$ & 0.260 \\
\hline$\frac{21}{2}^{+}$ & $\frac{5}{2}[622]$ & 0.242 & $\frac{23}{2}^{+}$ & $\frac{5}{2}[622]$ & 0.221 \\
& $\frac{1}{2}[651]$ & 0.285 & & $\frac{1}{2}[651]$ & 0.307 \\
\hline$\frac{25}{2}^{+}$ & $\frac{5}{2}[622]$ & 0.203 & $\frac{27}{2}^{+}$ & $\frac{5}{2}[622]$ & 0.187 \\
& $\frac{1}{2}[651]$ & 0.326 & & $\frac{1}{2}[651]$ & 0.342 \\
\hline$\frac{29}{2}^{+}$ & $\frac{5}{2}[622]$ & 0.175 & $\frac{31}{2}^{+}$ & $\frac{5}{2}[622]$ & 0.162 \\
& $\frac{1}{2}[651]$ & 0.355 & & $\frac{1}{2}[651]$ & 0.367 \\
\hline$\frac{33}{2}^{+}$ & $\frac{1}{2}[651]$ & 0.378 & $\frac{35}{2}^{+}$ & $\frac{1}{2}[651]$ & 0.387 \\
\hline$\frac{37^{+}}{2}$ & $\frac{1}{2}[651]$ & 0.393 & $\frac{39}{2}^{+}$ & $\frac{1}{2}[651]$ & 0.399 \\
\hline$\frac{41}{2}^{+}$ & $\frac{1}{2}[651]$ & 0.404 & $\frac{43}{3}^{+}$ & $\frac{1}{2}[651]$ & 0.407 \\
\hline$\frac{45}{2}^{+}$ & $\frac{1}{2}[651]$ & 0.411 & $\frac{47}{2}^{+}$ & $\frac{1}{2}[651]$ & 0.412 \\
\hline$\frac{49}{2}+$ & $\frac{1}{2}[651]$ & 0.413 & & & \\
\hline
\end{tabular}

around $I=\frac{21}{2}^{+}$(here the $\frac{1}{2}[651]$ orbital becomes dominant).

As can be seen in Figure 3.8, the aligned angular momentum extracted from the calculation is much greater than experimentally observed, a problem with 
some calculations with the cranking model. As the experimental data indicate rather small alignment varying smoothly at higher spins, a calculation was performed to suppress band crossings (or similarly a change in the character of the band). Increasing $\Delta$ enlarges the gap between orbitals. Letting $\Im_{c} / \hbar^{2}=$ $50 \mathrm{MeV}^{-1}$ reduces the amount of angular momentum carried by the valence particles, thus weakening the interactions between the orbitals. Both effects should conspire to suppress band crcisings. Increasing $\Delta$ and $\Im_{c}$ changes the $I=\frac{s}{2}^{+}$configuration to $17 \% \frac{1}{2}[640], 35 \% \frac{1}{2}[651]$, and $23 \% \frac{1}{2}[600]$, a configuration with a small amount of angular momenturn car ${ }^{*} .{ }^{\prime}$ oddneutron. It should be noted that the $K=\frac{1}{2}$ character of these lower spin states in this particular calculation is exactly as observed in the rotationa! band fits below, and it is the source of the staggering terms found essential in the fitting schemes. At $I=\frac{49}{2}{ }^{+}$, the configuration is $17 \% \frac{2}{2}[651], 17 \% \frac{3}{2}[651]$, and $23 \% \frac{3}{2}[642]$ indicating a band crossing, a feature that is persistent in all calculations. Since the cranking model does not fit the experimental energy levels very well, other rotational band fitting methods are presented below.

It has long been known that in the perturbation limit the effect of Coriolis band mixing $\left(K \neq \frac{1}{2}\right)$ in odd-A deformed nuclei is to renormalize the momentof-inertia, while leaving the $l(I+1)$ level spacing form unchanged. In the 


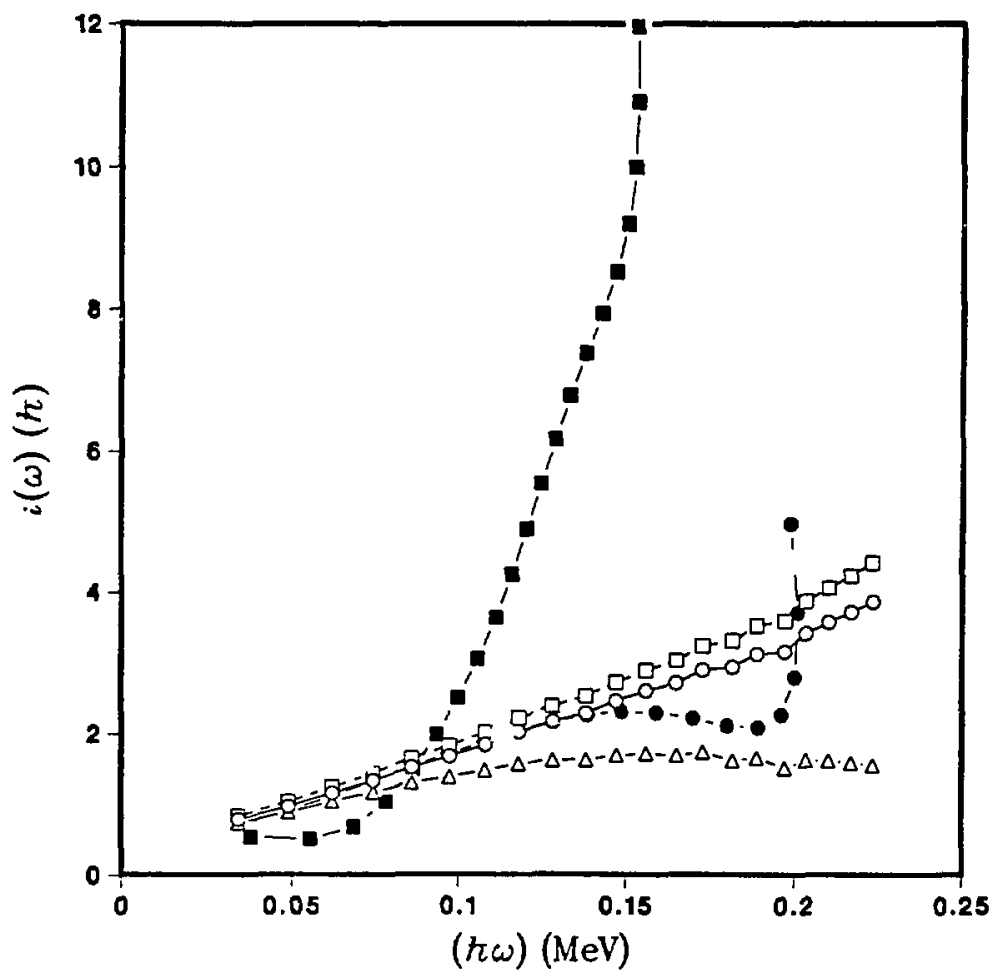

Figure 3.8: A comparison of extracted aligned anguar momentum for the ground rotational band in ${ }^{233} \mathrm{U}$ between experiment and cranking model calculations. The open symbols are the experimental values derived from the three reference states discussed in the text ( 0 is average of ${ }^{232} \mathrm{U}$ and ${ }^{236} \mathrm{U}$, open squares is ${ }^{236} \mathrm{U}$, and open triangles is ${ }^{231} \mathrm{U}$ ). The closed symbols are cranking model calculations using the standard parameters given in the text (closed squares) and increased $\beta_{2}$ parameter (•). 
other limit of two-band Coriolis mixing matrix elements large compared to zero order band separation, we get a constant aligned spin, $i_{\alpha}$,

$$
E(I)=\frac{\hbar^{2}}{2 \Im}\left(I-i_{\alpha}\right)^{2},
$$

with $i_{\alpha}=$ intrinsic mixing matrix element $\left\langle K+1\left|j_{x}\right| K\right\rangle$. The aligned spin formulation is equivalent to the introduction of a linear $I$ term into the power series expansion for energy levels. We extract $i_{\alpha}$ using the method of Bengtsson and Frauendorf [54]. The projection of the total angular momentum on the axis of rotation is given by

$$
I^{x}(I)=\sqrt{\left(I+\frac{1}{2}\right)^{2}-K^{2}}
$$

where $K$ is the projection of $I$ on the symmetry axis. The cranking frequency is detcrmined by

$$
\omega(I)=\frac{E(I+1)-E(I-1)}{I^{x}(I+1)-I^{x}(I-1)} .
$$

The aligned angular momentum is then

$$
i_{a}(\omega)=I^{x}(\omega)-I_{r e f}^{x}(\omega),
$$

where the reference

$$
I_{\mathrm{re} f}^{x}(\omega)=\omega \Im_{0}+\omega^{3} \Im_{1},
$$


and $\Im_{0}$ and $\Im_{1}$ are determined from nearest even-even neighbors. For the results presented in Figure 3.8, three reference states are chosen. The first reference state has $\Im_{0}$ and $\Im_{1}$ taken from the weighted average of ${ }^{232} \mathrm{U}$ and ${ }^{236} \mathrm{U}\left({ }^{234} \mathrm{U}\right.$ was excluded as $\Im_{1}$ is at a local maximum in that nucleus), and are $67.2 \hbar^{2} / \mathrm{MeV}$ and $44 \mathrm{~S}^{4} / \mathrm{MeV}^{3}$, respectively. The other two reference states have moments-of-inertia taken from ${ }^{234} \mathrm{U}\left(\Im_{0}=68.4 \hbar^{2} / \mathrm{MeV}, \Im_{1}=630 \hbar^{4} / \mathrm{MeV}^{3}\right)$ and ${ }^{236} \mathrm{U}\left(\Im_{0}=65.9 \hbar^{2} / \mathrm{MeV}, \Im_{1}=425 \hbar^{4} / \mathrm{MeV}^{3}\right)$ only. Figure 3.8 shows that the aligned angular momentum is a rather smoothly increasing as a function of spin. The rather sudden alignment observed in ${ }^{238} \mathrm{U}$ and ${ }^{235} \mathrm{U}$ in Ref. [53] is not observed here. As can be seen, the average of ${ }^{232} \mathrm{U}$ and ${ }^{236} \mathrm{U}$ is very similar to ${ }^{236} \mathrm{U}$ only as refe nce. The ${ }^{234} \mathrm{U}$ has a significant difference at high spins, where $i_{\alpha}$ saturates, due to the fact that ${ }^{234} \mathrm{U}$ is known to have a lot of neutron S-band character. This suggests that perhaps at high spins in ${ }^{233} \mathrm{U}$, there is a lot of neutron S-band character that isn't present at lower spins. The cranking calculation tends to overestimate the amount of aligned angular momentum.

Clearly the aligned angular momentum is not constant; thus, the simple limiting case of equation (3.4) is not valid. Our rotational band fitting scheme was then guided by attempts to fit to the simple limiting case (see equation (3.9) below) and then to fit the more complex intermediate cases, using semi- 
empirically derived fitting functions.

Rotational band fitting was more difficult than initially expected. Initial attempts to fit the band with:

$$
E=a_{0}+\sum_{i=1}^{\infty} a_{i}\left[I(I+1)-K^{2}\right]^{i / 2}
$$

and doing separate fits to the two signatures to avoid explicit introduction of a staggering term related to Coriolis interaction with "anomalous" $K=\frac{1}{2}$ bands, proved to be unsatisfactory in dealing with this reversing of the stagger previously noted. Fits were then attempted using explicitly introduced staggering terms and functions of the form:

$$
E(I)=D+\sum_{i=\text { odd }} A_{i} I^{i}+\sum_{j} B_{j}[I(I+1)]^{j}+(-1)^{I+\frac{5}{2}} C_{\frac{3}{2}} \frac{\left(I+\frac{5}{2}\right) !}{\left(I-\frac{5}{2}\right) !}
$$

where $A_{i}, B_{j}, C_{\frac{3}{2}}$, and $D$ are the fitted parameters, and the $C_{\frac{3}{2}}$ term is a staggering term between the two signatures ( $K=\frac{5}{2}$ band). The residuals of fits of this form were fairly good at the high end of the band, but sy'stematically in error at the low end. Also, the fits did indicate that the staggering term was quite small, as previously noted from examination of Figure 3.5. Nevertheless, even though the overall fit quality was acceptable, satisfactory explanation of the reversing of the stagger was missing, and a more complex fitting function 
was postulated:

$$
\begin{aligned}
E(I)= & D+B_{1}[I(I+1)]+A_{1} I\left[1-\exp \left\{\frac{-(I+1)}{I_{d}}\right\}\right] \\
& +A_{3} I^{2}(I+1)\left[1-\exp \left\{\frac{-(I+1)}{I_{d}}\right\}\right]+B_{S 1}(-1)^{I-\frac{1}{2}} \frac{\left(I+\frac{5}{2}\right) !}{\left(I-\frac{5}{2}\right) !} \\
& +B_{S_{2}}(-1)^{I-\frac{1}{2}}\left[1-\exp \left\{\frac{-\left(I+\frac{5}{2}\right)\left(I-\frac{3}{2}\right)}{I_{d}^{2}}\right\}\right] \frac{\left(I+\frac{3}{2}\right) !}{\left(I-\frac{3}{2}\right) !}
\end{aligned}
$$

where $B_{1}, A_{1}, A_{3}, B_{S 1}, B_{S 2}$, and $D$ are the fitted parameters, and like terms in equation (3.10) have the same coefficients in equation (3.11). $I_{d}$ is some critical spin where the Coriolis mixing tends to become more important (at low spins, the $A_{1}$ and $A_{3}$ terms become smaller as the Coriolis mixing is less). As can be seen in equation (3.11), consistent with our formulation of Coriolis band mixing in the perturbation limit, there is an $I(I+1)$ term. The $A_{1}$ term is analogous to the introduction of the linear $I$ term in the power series from inclusion of $i_{\alpha}$, and $A_{3}$ is the $I^{3}$ term. Both $A_{1}$ and $A_{3}$ have exponential factors modifying them so that they have the proper low-spin limiting form of $[I(I+1)]^{\text {n. }}$. $B_{S 1}$ and $B_{S 2}$ are staggering terms introduced from higher order coupling into two $K=\frac{1}{2}$ bands with opposite-sign decoupling constants. It is the competition between these two terms that could fit the reversing of the stagger noted in Figure 3.5.

Equations (3.9), (3.10), and (3.11) were all used to attempt to fit the 
rotational band - totaling about 25 fits. The results of the fits from equation (3.10) and equation (3.11) are presented in Table 3.4. As can be seen, the Table 3.4: ${ }^{233} \mathrm{U}$ rotational band fit parameters $(\mathrm{keV})$

\begin{tabular}{|c|c|c|c|c|c|c|c|}
\hline $\begin{array}{c}\text { Eq. } \\
(3.19)\end{array}$ & $D$ & $A_{1}$ & $A_{3}$ & $B_{1}$ & $\begin{array}{c}B_{2} \\
\left(\times 10^{5}\right)\end{array}$ & $\begin{array}{c}C_{\frac{3}{2}} \\
\left(\times 10^{7}\right)\end{array}$ & $\chi_{\nu}^{2}{ }^{\dagger}$ \\
\hline 1 & -46.29 & $-3.5 i$ & -0.05 & 6.39 & -4.8 & -2.3 & 0.114 \\
\hline 2 & -45.15 & -4.01 & -0.05 & 6.45 & - & -2.2 & 0.109 \\
\hline 3 & -46.81 & -3.46 & -0.05 & 6.40 & - & - & 0.724 \\
\hline $\begin{array}{c}\text { Eq. } \\
(3.11)\end{array}$ & $D$ & $A_{1}$ & $A_{3}$ & $B_{1}$ & $\begin{array}{c}B_{S 1} \\
\left(\times 10^{7}\right)\end{array}$ & $\begin{array}{c}B_{S 2} \\
\left(\times 10^{5}\right)\end{array}$ & $\chi_{\nu}^{2}$ \\
\hline 4 & -49.99 & -5.55 & -0.05 & 6.61 & -1.84 & 2.29 & 0.110 \\
\hline 5 & -50.09 & -5.47 & -0.05 & 6.60 & - & 11.7 & 0.119 \\
\hline 6 & -49.97 & -5.57 & -0.05 & 6.61 & -2.27 & - & 0.105 \\
\hline 7 & -50.62 & -5.05 & -0.05 & 6.57 & - & - & 0.707 \\
\hline
\end{tabular}
Table 3.1; that the $\chi_{v}^{2}$ values are much less than unity indicates that the standard deviations on the energy levels are too conservative for the energy level differences governing the fitting

fits using equation (3.11) were marginally better ( $\lambda_{\nu}^{2}$ is slightly lower). The significance of $B_{S_{1}}$ and $B_{S 2}$ were tested. We did not include $I_{d}$ explicitly for variation, as that makes the least squares non-linear. Fittings were tried for values of $I_{d}$ of 3.5 and 4.5, chosen by inspection of Figure 3.6. The latter value gave lower $\chi_{v}^{2}$.

The fit results indicate that $B_{S 1}$ and $B_{S 2}$ do indced have opposite signs. 


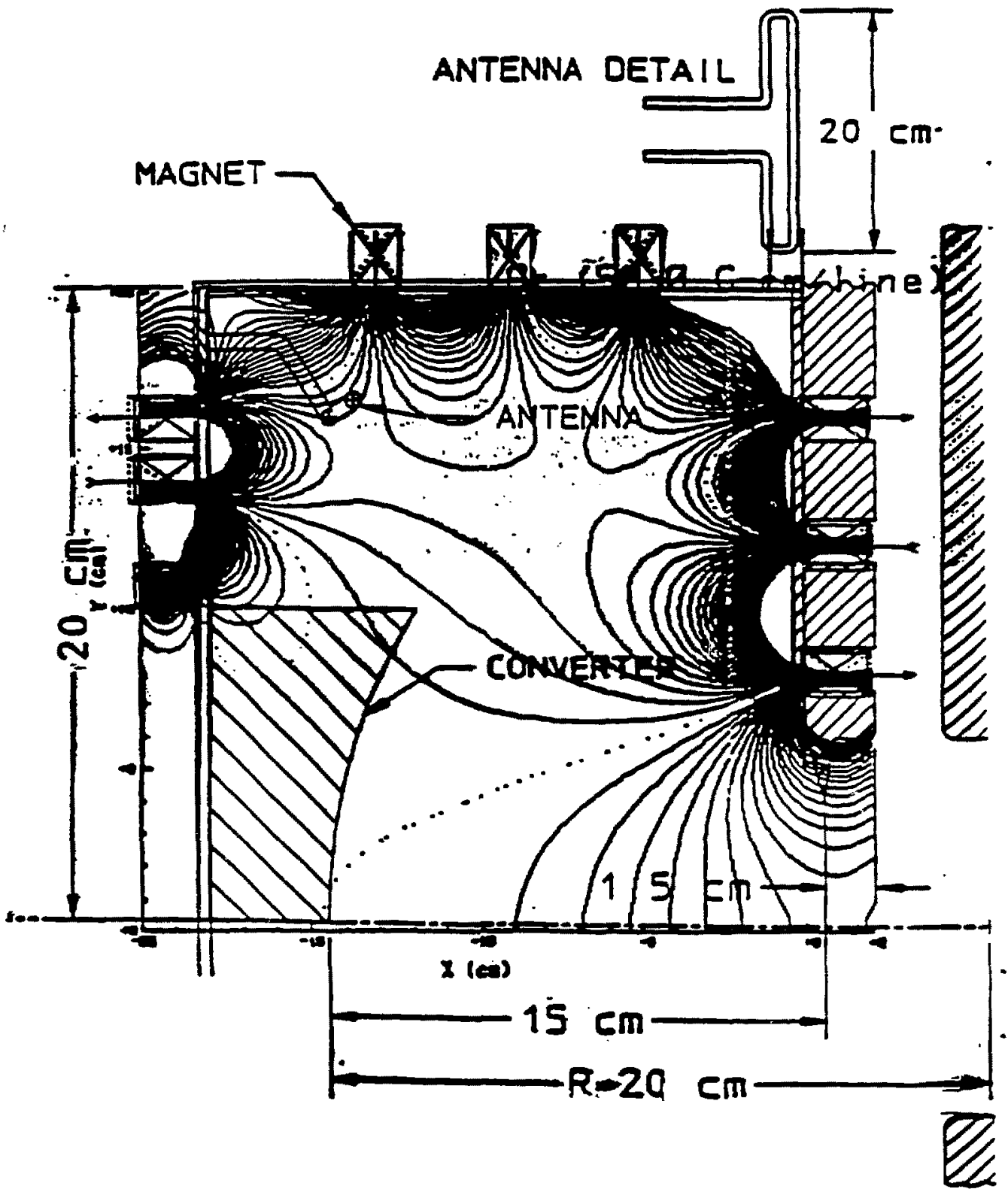

FIG. 11 SURFACE CONVERSION SOURCE MAGNETIC CALCULATIONS 
angular momentum,

$$
i_{\alpha}=\sqrt{[j(j+1)-\Omega(\Omega+1)]} .
$$

The aligned limiting expression has a linear term in spin $I$, as well as the quadratic term. This suggests that band energy fitting expressions need odd powers of $I$ at higher spins but should have only even powers of $I$ [or powers of $I(I+1)]$ at lowest spin. This problem became apparent to us in examining the residual errors from band fitting with expressions including a linear $I$ term.

The two-band mixing energies are analytical solutions of the quadratic equation from diagonalization of the $2 \times 2$ matrices. Fitting with such expressions has been done using non-linear least squares [56].

For analyzing the ${ }^{233} \mathrm{U}$ band power series we choose a slightly different approach, guided by the above experience. By inserting a factor $\left[1-\exp \left(I / I_{d}\right)\right]$ in front of the odd-powered spin terms, we get the desired behavior of only even powers in the expansion at the low end of the band, and the odd powers that go with aligned spin at the higher angular momenta. The problem of band fitting then becomes non-lincar in the term $I_{d}$, but it is still easy to use, with terms that have physical significance. We may identify $I_{d}$ with that spin at which the Coriolis matrix element of the leading band coupling becomes 
equal to the band separation. A more complete discussion of odd-A band fitting can be found in reference [57].

How does this apply to the staggering terms? It suggests that in addition to the Bohr-Mottelson perturbation term there might be terms with the above exponential factors to give the lower-power dependence at higher spins, where some Coriolis band coupling saturates. For a unique-parity orbital within a shell there will be only one $K=\frac{1}{2}$ band within the shell to which it can Corioliscouple. Thus, we would expect in such cases that the sense of staggering would be of a single sign. In the ${ }^{233} \mathrm{U}$ case, the ground-state band of $K=\frac{5}{2}^{+}$is a normal-parity orbital, and there are several $K=\frac{1}{2}$ bands within the shell available for Coriolis coupling, bands which have decoupling parameters of either sign. Consider a near-lying $K=\frac{1}{2}$ band with weak Coriolis coupling dow:: to ground and a higher-lying $K=\frac{1}{2}$ band with strong Coriolis coupling and opposite sign decoupling parameters. The lower band would dominate the staggering term at lower spin because of the smaller energy denominators in the perturbation expression, but at higher spin it would saturate, and the higher-lying band coupling would dominate. ${ }^{164} \mathrm{Er}$ is a nucleus in which both positive and negative parity bands exhibit a reversal of staggering [58].

Quasiparticle (single particle) energy levels (Routhians) were extracted 
from the ${ }^{233} \mathrm{U}$ ground-state band energy levels using the standard methods of Bengtsson and Frauendorf [59]. The Routhian is defined as:

$$
e^{\prime}=E^{\prime}(A, \omega)+\Delta-\frac{1}{2}\left[E_{\text {res }}^{\prime}(A+1, \omega)+E_{\text {res }}^{\prime}(A-1, \omega)\right],
$$

where $E^{\prime}(A, \omega)$ is given by:

$$
E^{\prime}(A, \omega)=E^{\prime}(I)=\frac{1}{2}(E(I+1)+E(I-1))-\omega(I) I_{x}(I)
$$

$I_{x}(I)$ is expressed by:

$$
I_{x}(I)=\sqrt{\left(I+\frac{1}{2}\right)^{2}-K^{2}}
$$

and $\omega(I)$ is:

$$
\omega(I)=\frac{E(I+1)-E(I-1)}{I_{x}(I+1)-I_{x}(I-1)} .
$$

The pairing gap parameter, $\Delta$, is chosen in the standard way where:

$$
\Delta=0.12 \hbar \omega_{0}
$$

and $\hbar \omega_{c}=\frac{41}{A^{\jmath}}$. The reference state is chosen as an average of neighboring even-even nuclei.

$$
E_{\text {res }}^{\prime}=-\frac{1}{2} \omega^{2} \Im_{0}-\frac{1}{4} \omega^{4} \Im_{1}+\frac{1}{8} \hbar^{2} / \Im_{0}
$$

Figure 3.9 shows the experimental Routhians, $e^{\prime}$, as a function of $\hbar \omega$ for three sets of reference states, (1) ${ }^{232} \mathrm{U}$ and ${ }^{234} \mathrm{U}$ averaged, (2) ${ }^{232} \mathrm{U}$ only, and 


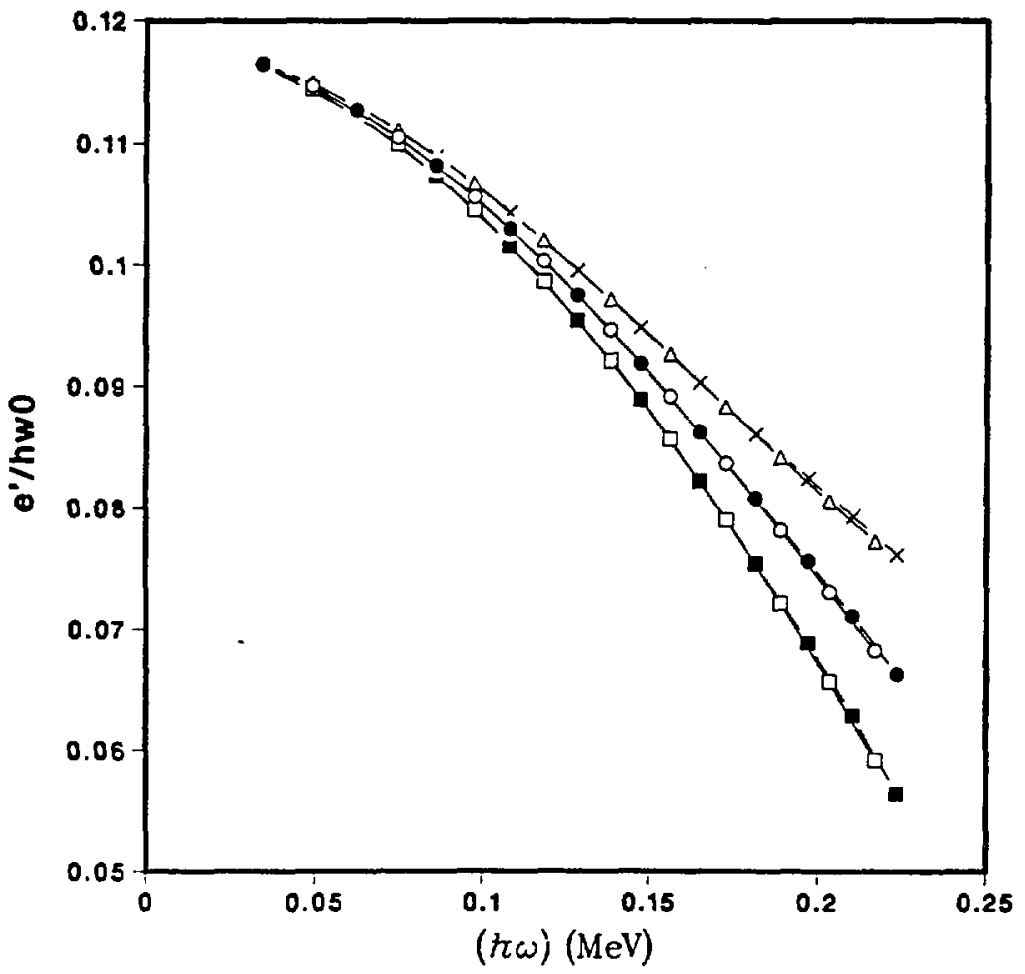

Figure 3.9: Experimental Routhians for the ground-state band in ${ }^{233} \mathrm{U}$. The open symbols are for the $\frac{5}{2}^{+}$signature and the closed symbols and X's are for the $\frac{7^{+}}{2}$ signature. Three reference states are shown: (1) ${ }^{232} \mathrm{U}$ and ${ }^{234} \mathrm{U}$ (open circles and $\bullet$ ), (2) ${ }^{232} \mathrm{U}$ only (open and closed squares), and (3) ${ }^{234} \mathrm{U}$ only (open triangles and X's). The choice of reference states does not affect the results much. 
(3) ${ }^{234} \mathrm{U}$ only. The Routhians, for all cases, decrease smoothly with increasing $\omega$, and both the favored and unfavored signature of the band tend to lie on one curve. This is consistent with the minimal signature splitting noted previously. Another cranking calculation was performed to examine the single particle levels. Figure 3.10 shows $e^{\prime}$ as a function of $\hbar \omega$ for a calculation with $N=$ $5,6,7$ neutron oscillator shells as a basis. At $\hbar \omega=0$, the level at $e^{\prime} / \hbar \omega_{0}=$ 0.1 is the $\frac{5}{2}^{+}[633]$ ground state. The first excited level is the $\frac{1}{2}^{+}[631]$ and the second excited level is the intruder state $\frac{7}{2}^{-}$. Several interesting things should be noted. Firstly, the ground-state band $\frac{5}{2}^{+}$level exhibits very little signature splitting, even at moderately high spins $\left(\hbar \omega / \hbar \omega_{0}=0.03\right)$. Secondly, the excited $\frac{1}{2}^{+}$band does exhibit much stronger signature splitting at high spins as is expected. Additionally, it appears as if one signature of the $\frac{1}{2}^{+}$ band crosses both signatures of the $\frac{5}{2}^{+}$band. This band crossing occurs at approximately the spin where the anomaly was noted in the moment-of-inertia figures, and would validate the explanation given above regarding the influence of the $K=\frac{1}{2}$ bands on the signature splitting. Please note, that at higher $\omega$. one signature of the $K=\frac{1}{2}^{+}$band is above and one below the $K=\frac{5}{2}^{+}$band (both signatures). Finally, however, note that this still does not completely explain the data. No reversal of staggering, as indicated by a crossing of 


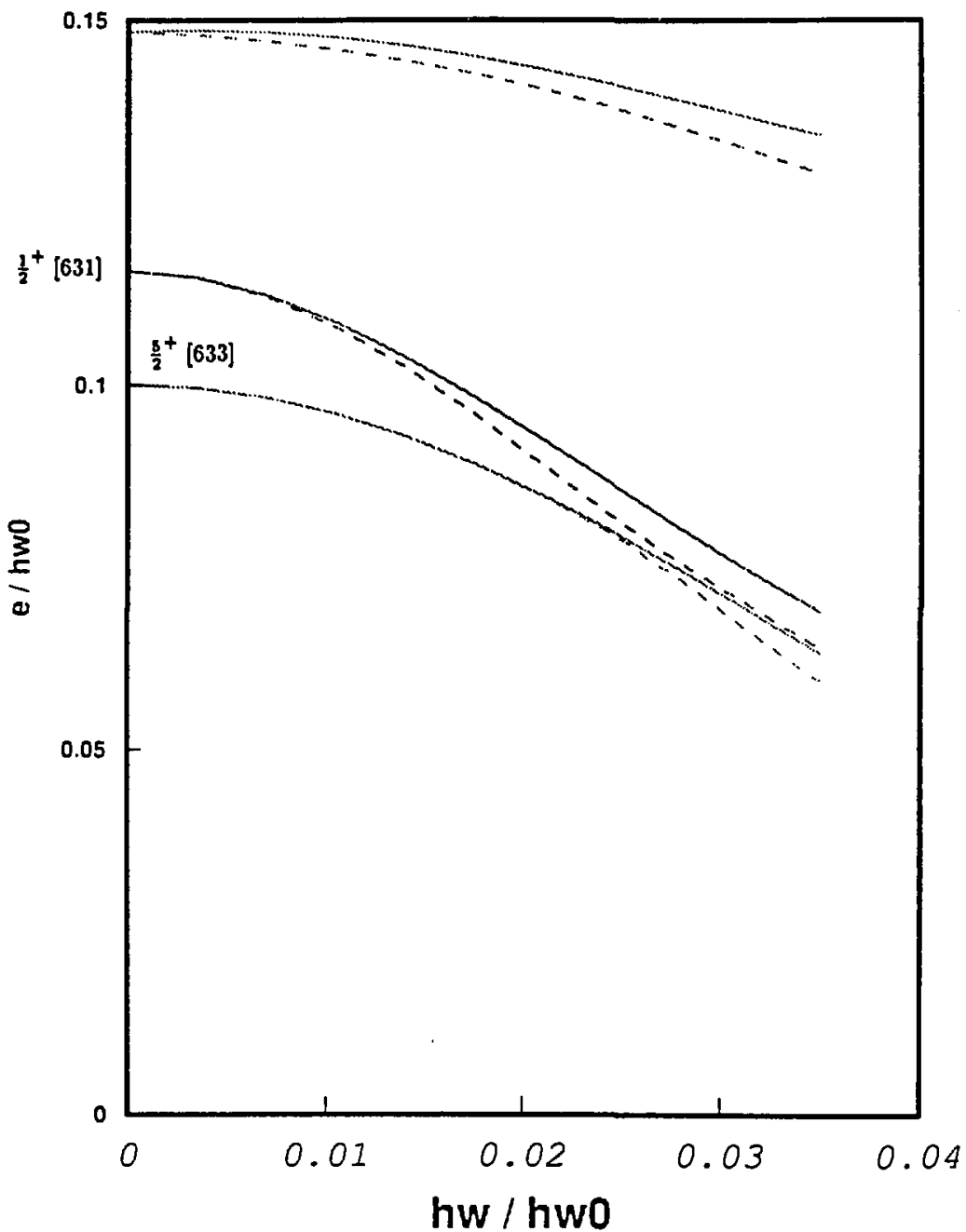

Figure 3.10: Routhians from cranking calculation for the ground-state band in ${ }^{233} \mathrm{U}$. The lowest level at $\hbar \omega=0$ is the $\frac{5}{2}^{+}$. ground state, the next excited level is the $\frac{1}{2}^{+}$band. The $\frac{7}{2}^{-}$intruder level has been removed for clarity. The favored signatures are the solid curves and the unfavored signatures are the dashed lines. 
signatures in a band, is observed.

Recently, however, signature splitting reversals have been explained by invoking $\gamma$-deformation or triaxial nuclear shapes [60]. Hamamoto shows for the $j=\frac{13}{2}$ shell in the rare-earths that for $\gamma=0^{\circ}$, the single particle levels just exhibit the normal splitting of favored and unfavored signatures (her Figure 1a), but for $\gamma=+20^{\circ}$ (her Figure 2a), the signatures cross $K-\frac{1}{2}$ times as a function of increasing $\omega$. Thus, a cranking calculation with a slight $\gamma$ deformation was performed. The results are shown in Figure 3.11 for a case where $\gamma=3^{\circ}$ (small $\gamma$-deformation) and $N=5,6$ neutrons are included. Again, at $\hbar \omega=0$ the ground-state level is the $\frac{5}{2}^{+}[633]$, and the first excited state is the $\frac{1}{2}^{+}$level. A larger signature splitting is observed for the $\frac{1}{2}^{+}$band at higher spins, as would be expected. However, several interesting differences occur. Firstly, the size of the splitting of signatures in the $\frac{5}{2}^{+}$band, while still small, is larger than that for the case of no $\gamma$-deformation. Secondly, the negative signature of the $\frac{1}{2}^{+}$band interacts strongly (and repels) the negative signature of the $\frac{5}{2}^{+}$ground-state band in the spin region where the anomalies in the moment-of-inertia were noted. In addition, there is no band crossing, but certainly a band interaction which must result in the change of character 


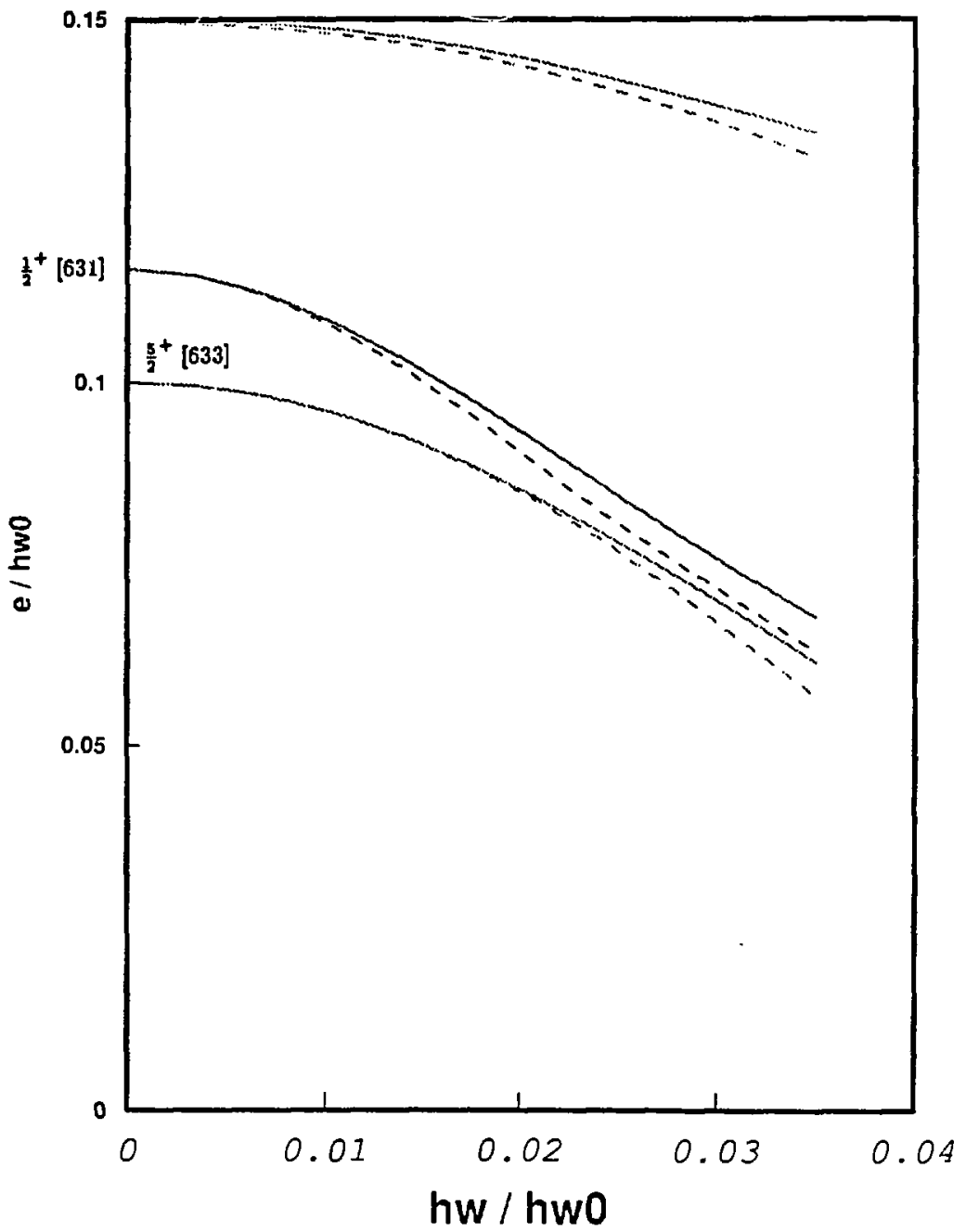

Figure 3.11: Routhians from cranking calculation for the ground-state band in ${ }^{233} \mathrm{U}$ including a slight $\gamma$-deformation. The levels and symbols are the same as in Figure 3.10. 
of the $\frac{5}{2}^{+}$band. Finally, at slightly higher spins, the favored and unfavored signatures of the $K=\frac{5}{2}^{+}$band cross, indicating a reversal of the staggering. This is in qualitative agreement with the data.

In conclusion, it seems that a slight triaxial shape deformation explains the anomalies in the moment-of-inertia and the reversal of staggering in the ground-state band in ${ }^{233} \mathrm{U}$. It might also explain the $\mathrm{g}$-factor information presented below. While none of the experimental evidence is overwhelming, this difficult-to-fit nucleus with these subtle effects seem to be best explained by a slight triaxiality induced at higher spins.

E0 transitions between $\mathrm{K}^{\pi}=0^{+}$bands in ${ }^{232} \mathrm{U},{ }^{234} \mathrm{U}$, and ${ }^{236} \mathrm{U}$ have been measured by others [61]. The E0 transition energies between an excited $K=0$ rotational band and the ground-state band in ${ }^{232} \mathrm{U}$ show the bands to be converging slightly with increasing spin (about $63 \mathrm{keV}$ closer at spin $10^{+}$than at spin $0^{+}$). However, in ${ }^{234} U$ the excited-band and the ground-state band are more parallel (only $14 \mathrm{keV}$ closer) indicating no real evidence for bandcrossing at these lower spins. Nevertheless, if the bands interact strongly, band crossings arc difficult to detect (there is no sharp change in the level spacings). Recent experiments [62] studying the heavy-ion, one-neutron transfer reaction 
product ${ }^{234} \mathrm{U}$ do indicate a possible band crossing in ${ }^{234} \mathrm{U}$ at about spin $20^{+}$, that is, near the same spin region as the $\Im^{(2)}$ irregularity noted above in ${ }^{233} \mathrm{U}$. These data further support the theoretical interpretation extracted from the Routhians.

Additional information can be obtained from the lower energy Coulomb excitation experiment with ${ }^{58} \mathrm{Ni}$ projectiles. Several lower energy cascade transitions were observed, besides the dominant E2 crossovers. The transition rates for gamma decay can be expressed in terms of the rec uced transition probabilities [63]):

$$
\begin{aligned}
& W(M 1)=1.71 \times 10^{13}\left(E_{\gamma}\right)^{3} B(M 1) \mathrm{sec}^{-1} \\
& W(E 2)=1.23 \times 10^{13}\left(E_{\gamma}\right)^{5} B(E 2) \mathrm{se}^{-1},
\end{aligned}
$$

where $E_{\gamma}$ is in $\mathrm{MeV}$. The cascade-to-crossover photon intensity ratio is given by:

$$
\frac{I_{c}}{I_{x}}=\frac{W(M 1)+W(E 2 ; I-I-1)}{W(E 2 ; I \rightarrow I-2)}
$$

where

$$
\begin{aligned}
& I_{\mathrm{c}}=\text { intensity of cascade } \gamma \text {-ray } \\
& I_{x}=\text { intensity of crossover } \gamma \text {-ray. }
\end{aligned}
$$


The reduced transition probability $\mathrm{B}(\mathrm{M} 1)$ is given by [64]:

$$
B(M 1)=\frac{I_{0}^{2}}{4.19}\left\langle I 1 K 0 \mid I 1 I-1 K^{2}\right\rangle^{2}\left(g_{\Omega}-g_{R}\right)^{2}
$$

where

$$
\begin{aligned}
& g_{\Omega}=\mathrm{g} \text {-factor of odd neutron } \\
& g_{R}=\mathrm{g} \text {-factor of core } \\
& I_{0}=\text { ground-state spin }\left(\frac{5}{2}^{+} \text {for }{ }^{233} \mathrm{U}\right) \\
& I=\text { spin of nucleus } \\
& K=\text { projection of spin on symmetry axis } \\
& \langle\mid\rangle^{2}=\text { square of the Clebsch-Gordan coefficient }
\end{aligned}
$$

and the reduced transition probabilities $B(E 2 ; I \rightarrow I-1)$ and $B(E 2 ; I \rightarrow I-2)$ are given by $[65]$ :

$$
\begin{aligned}
& B(E 2 ; I \rightarrow I-1)=\frac{5}{16 \pi} e^{2} Q_{0}^{2}\left\langle I 2 K^{\prime} 0 \mid I 2 I-1 K^{2}\right\rangle^{2} \\
& B(E 2 ; I \rightarrow I-2)=\frac{5}{16 \pi} e^{2} Q_{0}^{2}\left\langle I 2 K 0 \mid I 2 I-2 K^{\prime}\right\rangle^{2}
\end{aligned}
$$

where

$$
Q_{0}=\text { intrinsic quadrupole moment. }
$$

Combining equations (3.19)-(3.24), a relationship relating gyromagnetic factors and the quadrupole moment to experimentally measured quantities can be obtained: 


$$
\begin{aligned}
\left(\frac{g_{\Omega}-g_{R}}{Q_{0}}\right)^{2}= & \frac{0.4317}{I_{0}^{2}} \frac{\langle I 2 K 0 \mid I 2 I-2 K\rangle^{2}}{\langle I 1 K 0 \mid I 1 I-1 K\rangle^{2}} \\
& \left\{\frac{I_{c}}{I_{x}} \frac{E_{x}^{5}}{E_{c}^{3}}-E_{c}^{2} \frac{\langle I 2 K 0 \mid I 2 I-1 K\rangle^{2}}{\langle I 2 K 0 \mid I 2 I-2 K\rangle^{2}}\right\} \text { barns }^{-2}(3.2
\end{aligned}
$$

where

$$
\begin{aligned}
& E_{x}=\text { Energy of crossover } \gamma \text {-ray } \\
& E_{\mathrm{c}}=\text { Energy of cascade } \gamma \text {-ray. }
\end{aligned}
$$

The magnetic moment $\mu$ can be related to the $\mathrm{g}$-factors as follows [57]:

$$
\mu=\frac{I_{0}^{2}}{I_{0}+1} g_{\Omega}+\frac{I_{0}}{I_{0}+1} g_{\Omega}
$$

Cascade-to-crossover ratios for three low $\frac{M 1}{E_{2}}$ mixed transitions were determined, and if one assumes that $Q_{0}$ is relatively constant over this range of spins, then $g_{\Omega}-g_{R}$ can be extracted as one goes to higher spins. For ${ }^{233} \mathrm{U}$, $Q_{0}=11.55$ barns [66], $\mu= \pm 0.54$, and $I_{0}=\frac{5}{2}^{+}$(ref. [67]). Table 3.5 gives complete results for indicated cascade-to-crossover ratios extracted from the data. The $\frac{17}{2}^{+} \rightarrow \frac{15}{2}^{+}$transition is masked by uranium $X$-rays; thus, its $\frac{I_{c}}{I_{x}}$ ratio is determined by a linear interpolation. The projected values of $\frac{l_{\varepsilon}}{I_{x}}$ shown in Table 3.5 are derived from Clebsch-Gordan coefficients and the lowest mixing ratio as reference (the $40.35-\mathrm{eVV}^{\frac{7}{2}}+\frac{5}{2}^{+}$transition which has $\frac{M !}{E 2}$ ratio of 3.226 ) [ref. [68]]. The $\left(\frac{2 R-g_{8}}{Q_{0}}\right)^{2}$ value derived from projected $\frac{I_{c}}{T_{x}}$ ratios is 
Table 3.5: Apparent $\mathrm{g}$-factors deduced from cascade-to-crossover transition ratios for ${ }^{233} \mathrm{U}$

\begin{tabular}{|c|c|c|c|c|}
\hline $\begin{array}{l}\frac{M 1 E 2}{E 2} \\
\text { Ratio }\end{array}$ & $\frac{I_{c}}{l_{s}}($ proj. $)$ & $\frac{I_{f}}{T_{z}}(\exp )$ & $\begin{array}{c}\left(\frac{g n-g R}{Q_{0}}\right)^{2} \\
\left(\times 10^{4} \mathrm{barns}^{-2}\right)\end{array}$ & $\left|g_{\Omega}-g_{\boldsymbol{R}}\right|$ \\
\hline 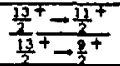 & 0.18 & $0.28 \pm 0.010$ & $10.7 \pm 0.46$ & $0.38 \pm 0.05$ \\
\hline$\frac{\frac{13}{2}+-\frac{13}{3}+}{\frac{13}{2}+\frac{31}{2}+}$ & 0.11 & $0.13 \pm 0.013$ & $7.05 \pm 0.90$ & $0.31 \pm 0.05$ \\
\hline$\frac{\frac{177^{+} \rightarrow \frac{15}{2}}{2}}{\frac{17^{+}}{2}-\frac{13^{7}}{2}}$ & 0.09 & $0.08 \pm 0.010^{\dagger}$ & $5.60 \div 0.50^{\dagger}$ & $0.27 \pm 0.04$ \\
\hline $\begin{array}{l}\frac{19}{2}+\rightarrow \frac{17}{2}+ \\
\frac{19}{2}+\frac{13}{2}^{+}\end{array}$ & 0.06 & $0.04 \pm 0.005$ & $3.10 \pm 0.65$ & $0.20 \pm 0.03$ \\
\hline
\end{tabular}

$f$ Cascade masked by uranium X-ray, $\frac{I_{c}}{I_{x}}$ obtained by interpolation

$6.04 \times 10^{-4}$ barns $^{-2}$. If one makes the assumption that the magnetic moment docs not change as spin increases, one can extract individual $g_{\Omega}$ and $g_{R}$ factors. However, the experimentally determined $\left(\frac{g_{n}-g_{R}}{Q_{0}}\right)^{2}$ ratios decrease rather strongly with increasing spin, implying that the effective values of $g_{\Omega}-g_{R}$, and thus the magnetic moment $\mu$, vary with increasing spin. The simplest rotational theory estimates the rotational g-factor of the core to be just the ratio of protons to all nucleons, $g_{R} \simeq \frac{Z}{A}=0.395$ for ${ }^{233} \mathrm{U}$. The g-factors derived from the projected $\frac{l_{c}}{l_{x}}$ values are $g_{\Omega}=0.13$ and $g_{R}=0.42$. The changing experimental $\mathrm{g}$-factors are indicative of Coriolis band mixing effects driving the increase in moment-of-inertia, as discussed above regarding the rotational band fits. The average $g_{R}$ value is 0.42 , slightly higher than simple theory 
predicts, and the average $g_{\Omega}$ value is 0.13 . Both $\mathrm{g}$-factors are consistent with those derived and tabulated by Chasman et al. [69], $g_{\Omega}=0.08$ and $g_{R}=0.39$, and from projections using Clebsch-Gordan coefficients. The average value of $\left(\frac{2 n-g_{R}}{Q_{0}}\right)^{2}=6.61 \times 10^{-4}$ barns $^{-2}$, gives $\frac{28-g_{R}}{Q_{0}}=0.026$ barns $^{-1}$, which is three times larger than the value reported by Newton [70] of 0.008 for the lowest cascade-to-crossover ratio. As discussed above, the moments-of-inertia vary rather smoothly in this lower spin region, and it appears that the magnetic moment varies also.

\section{3 ${ }^{90} \mathrm{Zr}+{ }^{239} \mathrm{Pu}$ Reaction}

The wealth of data on ${ }^{239} \mathrm{Pu}$ and ${ }^{238} \mathrm{Pu}$ will be discussed in this section. Figure 3.12 shows a total $\gamma$-ray spectrum for all 18 Ge detectors appropriately Doppler corrected. The spectrum is dominated by inelastic ${ }^{239} \mathrm{Pu}$ transitions and oneneutron transfer ${ }^{238} \mathrm{Pu}$ transitions. Very few fission events were observed. The neutron multiplicity for this reaction is shown in Figure 3.13. Events with $K_{n}=0$ occurred $40 \%$ of the time. Events with $K_{n}=1$ happened $\sim 25 \%$ of the time, and as clearly shown in Figure 3.13, events with higher neutron multiplicities were recorded ( $K_{n}=8$ occurred $\sim 1 \%$ of the time). These events 




Figure 3.12: Total Ge $\gamma$-ray spectrum for the ${ }^{90} \mathrm{Zr}+{ }^{239} \mathrm{Pu}$ reaction. Several ${ }^{239} \mathrm{Pu}$ peaks and one ${ }^{238} \mathrm{Pu}$ peak are marked. The peaks around $100 \mathrm{keV}$ are due to Pu $x$-rays. Note the absence of fission background. The arrows mark the peak positions of the mystery band. 


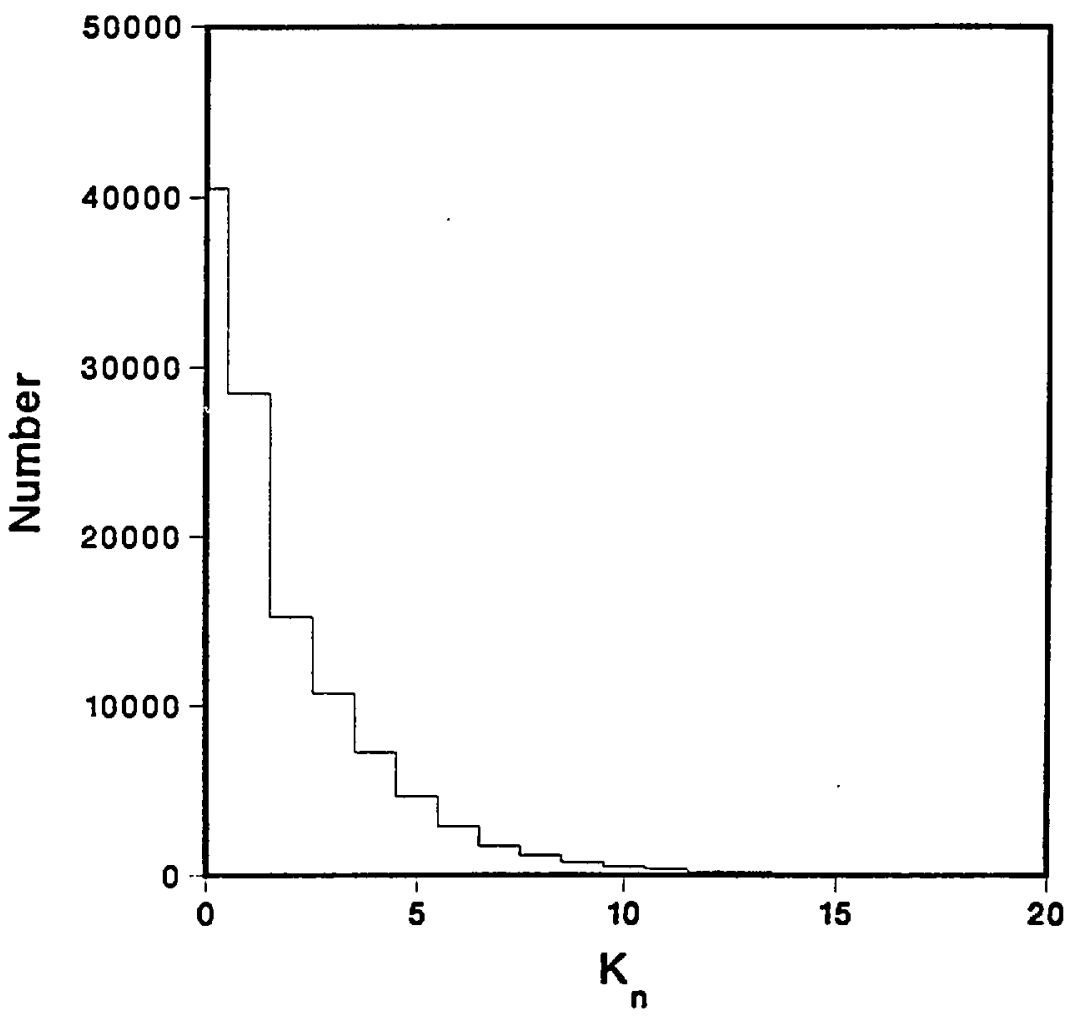

Figure 3.13: Neutron multiplicity for the ${ }^{90} \mathrm{Zr}+{ }^{239} \mathrm{Pu}$ reaction. 
are due to fission and neutron evaporation reactions, but a gate on $K_{n}=0$ suppresses both neutron evaporation and fission. A series of mystery transitions is also observed in this spectrum (marked with arrows). These mystery transitions appear in a regularly spaced manner consistent with a rotational band with a similar dynamic moment-of-inertia as the ground rotational band in ${ }^{238} \mathrm{Pu}$. They will be discussed further below.

Figure 3.14 shows a two-dimensional plot of the total $\gamma$ - $\gamma$ coincidence spectrum, $E_{\gamma 1}$ vs. $E_{\gamma 2}$. Dark spots indicate peak positions. The essential features of Figure 3.14 are as follows: (1) Peaks around $100 \mathrm{keV}$ correspond to $\mathrm{Pu} \mathrm{x}$-rays, and are in coircidence with both ${ }^{239} \mathrm{Pu}$ and ${ }^{238} \mathrm{Pu}$ transitions, as well as themselves (multjple $\mathrm{x}$-rays can be emitted per event); (2) The characteristic doublet of ${ }^{239} \mathrm{Pu}$ ground-state band transitions from both signatures is clearly observed (transitions appear like a doublet because staggering is so large); (3) ${ }^{238} \mathrm{Pu}$ transitions are clearly identifiable starting at the $307-\mathrm{keV} 12^{+} \rightarrow 10^{+}$ transition (lower ones are masked by ${ }^{239} \mathrm{Pu}$ peaks); (4) The $45^{\circ}$ axis is devoid of peaks, as expected, because no particular $\gamma$-ray is in coincidence with itself; (5) The streaks between clear peaks are due te unsuppressed Compton scattering events; thus, the peaks sit on top of background ridges; (6) The mystery band mentioned previously can be clearly identified (see arrows); (7) The 


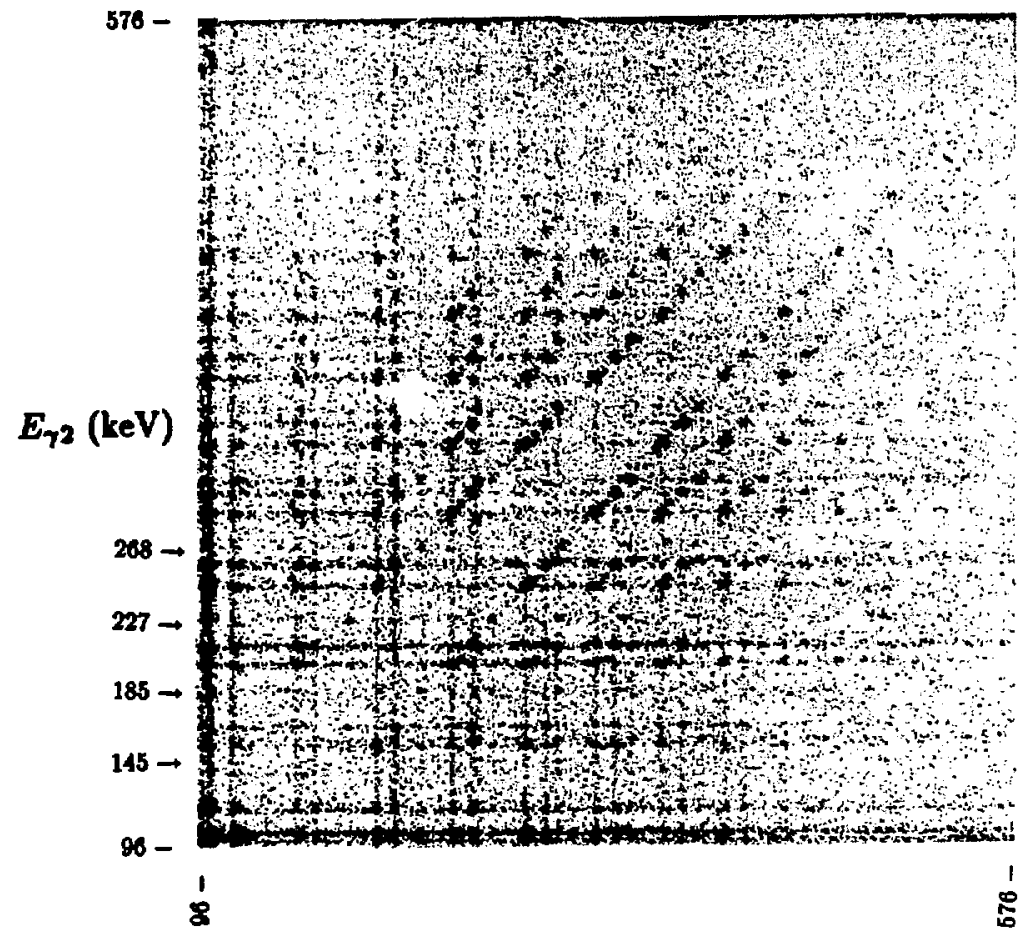

\section{$E_{\gamma 1}(\mathrm{keV})$}

Figure 3.14: Total two dimensional Ge $\gamma$ - $\gamma$ coincidence spectrum for the ${ }^{90} \mathrm{Zr}+$ ${ }^{239} \mathrm{Pu}$ reaction showing relationships between $\gamma$-rays in this experiment. Both inelastic ${ }^{239} \mathrm{Pu} \gamma$-rays and one-neutron transfer ${ }^{238} \mathrm{Pu} \gamma$-rays are present. 
mystery transition at $227 \mathrm{keV}$ is in coincidence with the $307-\mathrm{keV} 12^{+} \rightarrow 10^{+}$ ${ }^{238} \mathrm{Pu}$ transition, indicating that the mystery transitions are in ${ }^{238} \mathrm{Pu}$; and (8) Slices of this two-dimensional spectrum will show the rotational bands to be investigated.

Figure 3.15 shows a plot of the total $(H, K)$ spectrum for this reaction. Projections of the $H$ and $K$ for the reaction are shown in Figure 3.16. Figures 3.15 and 3.16 clearly show the average multiplicity to be $3-4$ and the average total energy to be $\sim 1 \mathrm{MeV}$. Furthermore, there is only one maximum located at lower $\left(H, K^{\prime}\right)$, indicating that perhaps the two quasi-particle transfer reaction cross-section is suppressed. The $\left(H, K^{\circ}\right)$ spectrum gated on inelastic transitions will be discussed in Section 3.3.I, and $(H, K)$ gated on transfer will be discussed in Section 3.3.2.

\subsection{1 ${ }^{239} \mathrm{Pu}$}

Figure 3.17 shows a -ray spectrum gated on four ${ }^{239} \mathrm{Pu}$ transitions; the 201.1$\mathrm{keV}{\frac{17^{+}}{2}}^{+} \rightarrow \frac{13}{2}^{+}, 212.1-\mathrm{keV} \frac{19}{2}^{+} \rightarrow \frac{15}{2}^{+}, 288.3-\mathrm{keV} \frac{25}{2}^{+} \rightarrow \frac{21}{2}^{+}$and $299.7-\mathrm{keV}$ $\frac{27}{2}^{+} \rightarrow \frac{23}{2}^{+}$transitions. The ground rotational band in ${ }^{239} 1 \mathrm{u}$ is observed up to the $500-\mathrm{keV} \frac{49}{2}^{+} \rightarrow \frac{45}{2}^{+}$transition, at least $9 h$ higher thai: previously known 


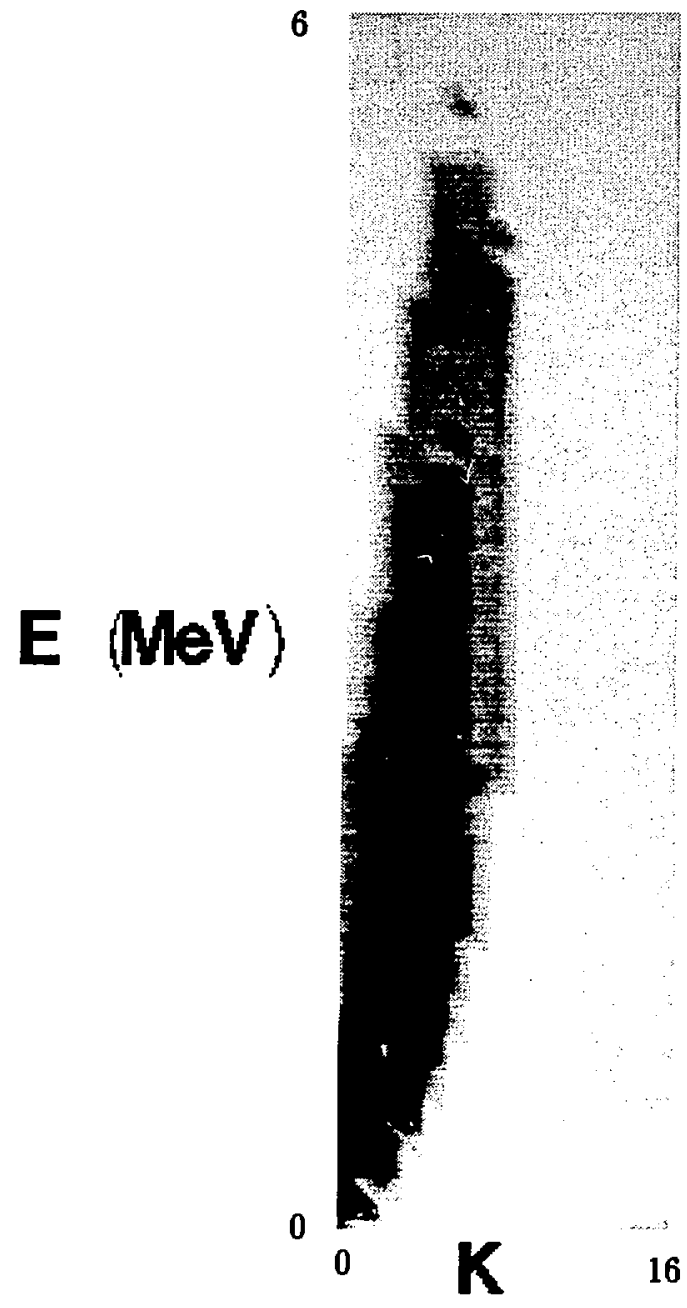

Figure 3.15: Total $(H, K)$ spectrum for the ${ }^{90} \mathrm{Zr}+{ }^{239} \mathrm{Pu}$ reaction. 

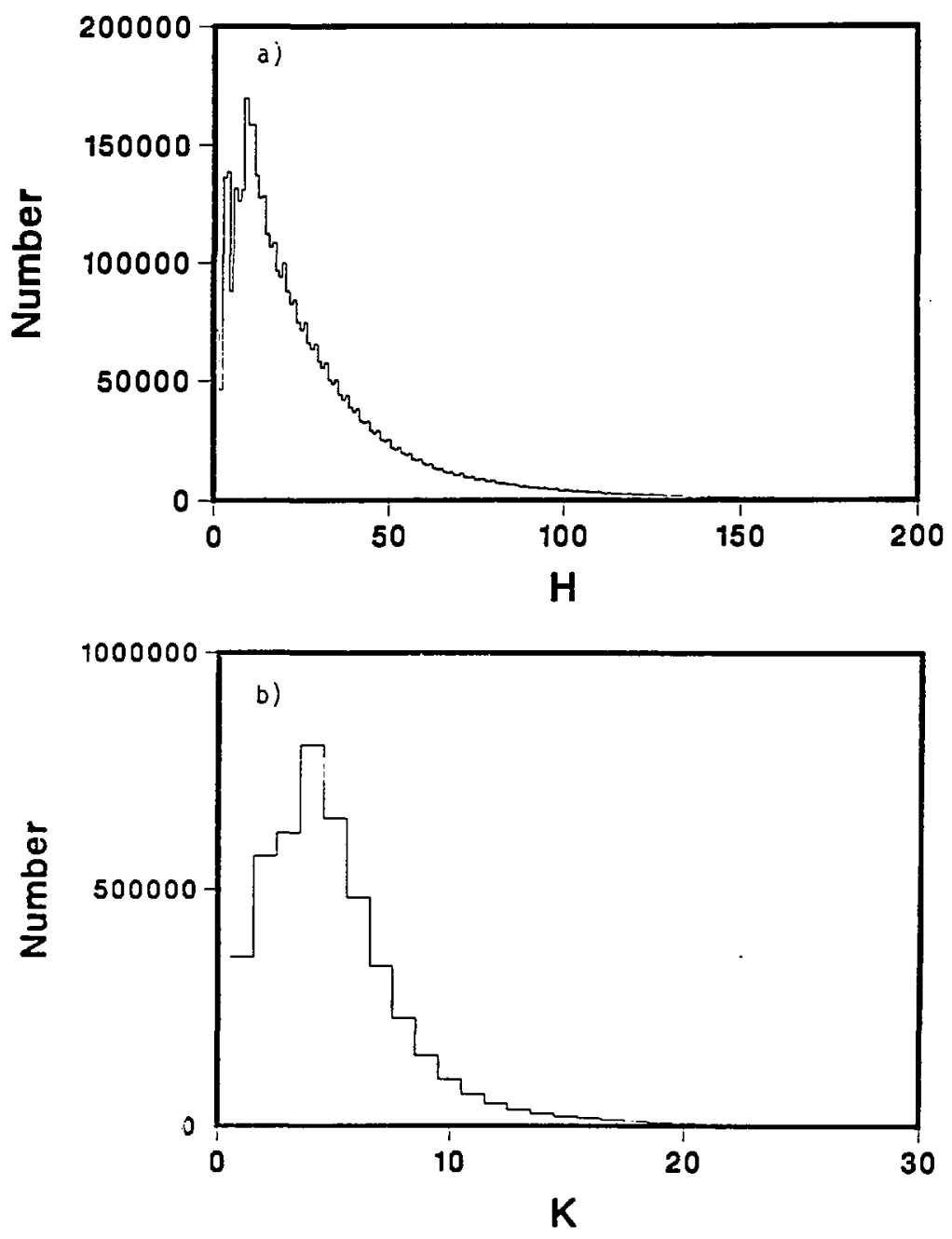

Figure 3.16: Projection of a) $H$ and b) $K$ for the ${ }^{90} \mathrm{Zr}+{ }^{239} \mathrm{Pu}$ reaction. 


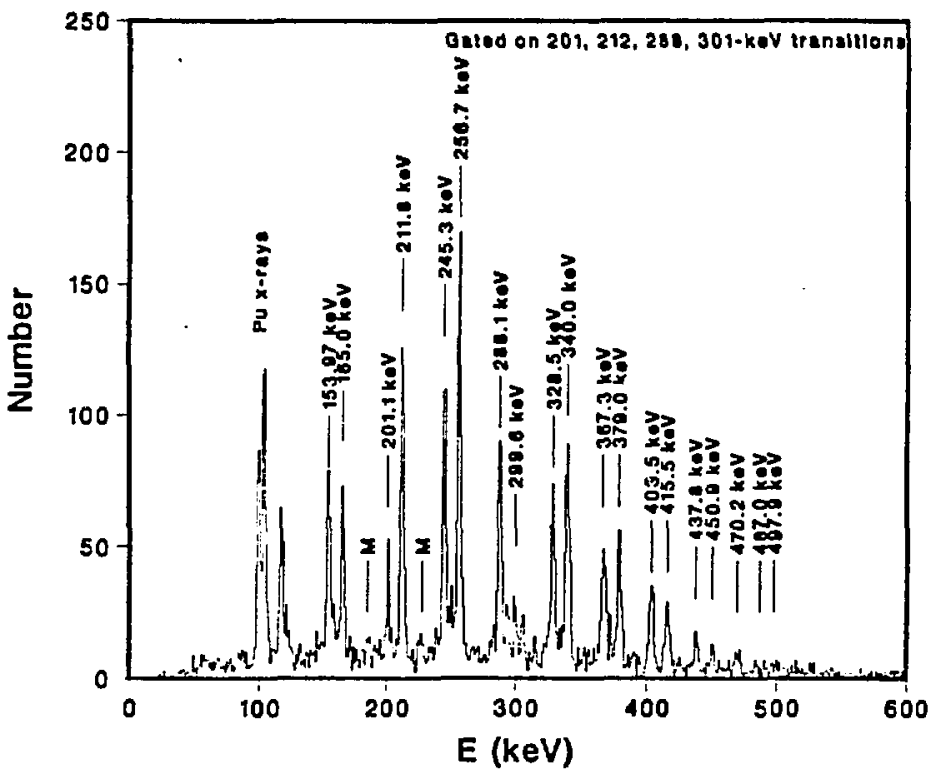

Figure 3.17: Ge $\gamma-\gamma$ spectrum for the ${ }^{90} \mathrm{Z} \tau+{ }^{239} \mathrm{Pu}$ reaction gated on the 201.1$\mathrm{keV} \frac{17}{2}^{+} \rightarrow \frac{13}{2}^{+}, 212.1-\mathrm{keV} \frac{19}{2}^{+} \rightarrow \frac{15}{2}^{+}, 288.3-\mathrm{keV} \frac{25}{2}^{+} \rightarrow \frac{21}{2}^{+}$and $299.7-\mathrm{keV}$ $\frac{27}{2}^{+} \rightarrow \frac{23}{2}^{+}$transitions. ${ }^{239} \mathrm{Pu}$ transitions are marked with their corresponding energies. Two "mystery" transitions discussed in the text are marked with II's. 
[41]. The band appears to be fairly regular, as shown by the transition energies given in Table 3.6. The moments-of-inertia are presented in Figure 3.18 for

Table 3.6: Observed transitions in ${ }^{239} \mathrm{Pu}$

\begin{tabular}{|c|c|c|c|}
\hline Transition & Energy (keV) & Transition & Energy (keV) \\
\hline & $117.1(2)$ & & $153.97(20)$ \\
\hline & $165.0(2)$ & & $201.1(2)$ \\
\hline & $211.8(2)$ & & $245.3(2)$ \\
\hline & $256 . i(2)$ & & $288.1(2)$ \\
\hline & $299.6(2)$ & & $328.5(2)$ \\
\hline & $340.0(2)$ & & $367.3(4)$ \\
\hline$\overline{2}$ & $379.0(4)$ & & $403.5(4)$ \\
\hline & $415.5(4)$ & & $437.8(4)$ \\
\hline & $450.9(4)$ & $\frac{45}{2}$ & $470.2(4)$ \\
\hline$\frac{47}{2}^{+} \rightarrow \frac{43}{2}^{+}$ & $48 \pi .0(4)$ & $\frac{49}{2}+$ & $497.9(4)$ \\
\hline
\end{tabular}

both signatures of the ground-state band. The kinematic moment-of-inertia $\Im^{(1)}$ has an anomaly at low spin $(\hbar w \approx 0.05 \mathrm{MeV})$, but then smooths out and gently increases with increasing $\hbar \omega$. The kinematic moment-of-inertia, $\frac{2 \mathrm{~J}^{(1)}}{\hbar^{2}}$, is around $8.5 \mathrm{MeV}^{-1}$ in this rotational velocity range. The dynamic moment-uf-inertia, however, shows a rather large bre: $k$ at $\hbar \omega \approx 0.22 \mathrm{MeV}$, and has a much larger increase than $\Im^{(1)}\left(\frac{2 \mathcal{S}^{(2)}}{h^{2}}\right.$ increases from $\sim 74 \mathrm{MeV}^{-1}$ at grciand state to $\sim 140 \mathrm{MeV}^{-1}$ at the top of the band - about a factor of two increase). Figure 3.19, the jriverse moment-of-inertia $\mathcal{S}^{(1)}$ as a function of 




Figure 3.18: Moments-of-inertia for the ground-state band in ${ }^{239} \mathrm{Pu}$. The open symbols are for the $\frac{1}{2}^{+}$favored signature and the closed symbols are for the $\frac{3}{2}^{+}$ unfavored signature. The kinematic moment-of-inertia, $\Im^{(1)}$, (open circles and -) and the dynamic moment-of-inertia, $\Im^{(2)}$, (орел squares and solid squares) are shown as a function of cranking frequency. An anomaly in the dynamic moment-of-inertia is observed at $\omega \approx 0.22$. The kinematic moment-of-inertia has a strange behavior at lower spins whirh may be alignment. 
$I^{2}$, clearly shows a rather large staggering between the favored and unfavored signatures of the band, which is characteristic of a $K=\frac{1}{2}^{+}$band. Note that the difference between the favored signature $(\sim 6 \mathrm{keV})$ and the unfavored signature $(\sim 17 \mathrm{keV})$ is a factor of three, much larger than the ${ }^{233} \mathrm{U}$ case discussed in Section 3.2. No clear backbending or upbending is observed, but there is a discrepancy at higher spins. However, in this region a proton backbending is expected prior (lower $\hbar \omega$ ) to a neutron backbend. Because rotation is a collective effect, though, such a backbend might also be observed even though neutron quasi-particle states are excited.

The aligned angular momentum $\left(i_{c}\right)$ is extracted as before (see Section 3.2 ) and is presented in Figure 3.20 using the ${ }^{238} \mathrm{Pu} \Im_{0}=67.9 \hbar^{2} / \mathrm{MeV}$ and $\Im_{1}=370 \hbar^{4} / \mathrm{MeV}^{3}$ for the reference state. The alignment is smooth and constant for each signature, the staggering is large as noted before, and an anomalously low $i_{\alpha}$ is observed for the $\frac{47^{+}}{2}$ state at $\hbar \omega \approx 0.24 \mathrm{MeV}$. The moment-of-inertia data and the aligned angular momentum data indicate a possible band crossing point at $\hbar \omega \approx 0.22-0.24 \mathrm{MeV}$.

Single particle energy level Routhians for the ground-state band in ${ }^{239} \mathrm{Pu}$ were also extracted from the data as in Section 3.2 and are presented in Figure 3.21 for a ${ }^{238} \mathrm{Pu}$ reference state $\left(\Im_{0}=67.9 h^{2} / \mathrm{MeV}\right.$ and $\left.\Im_{1}=320 \hbar^{4} / \mathrm{Mev}^{3}\right)$. 


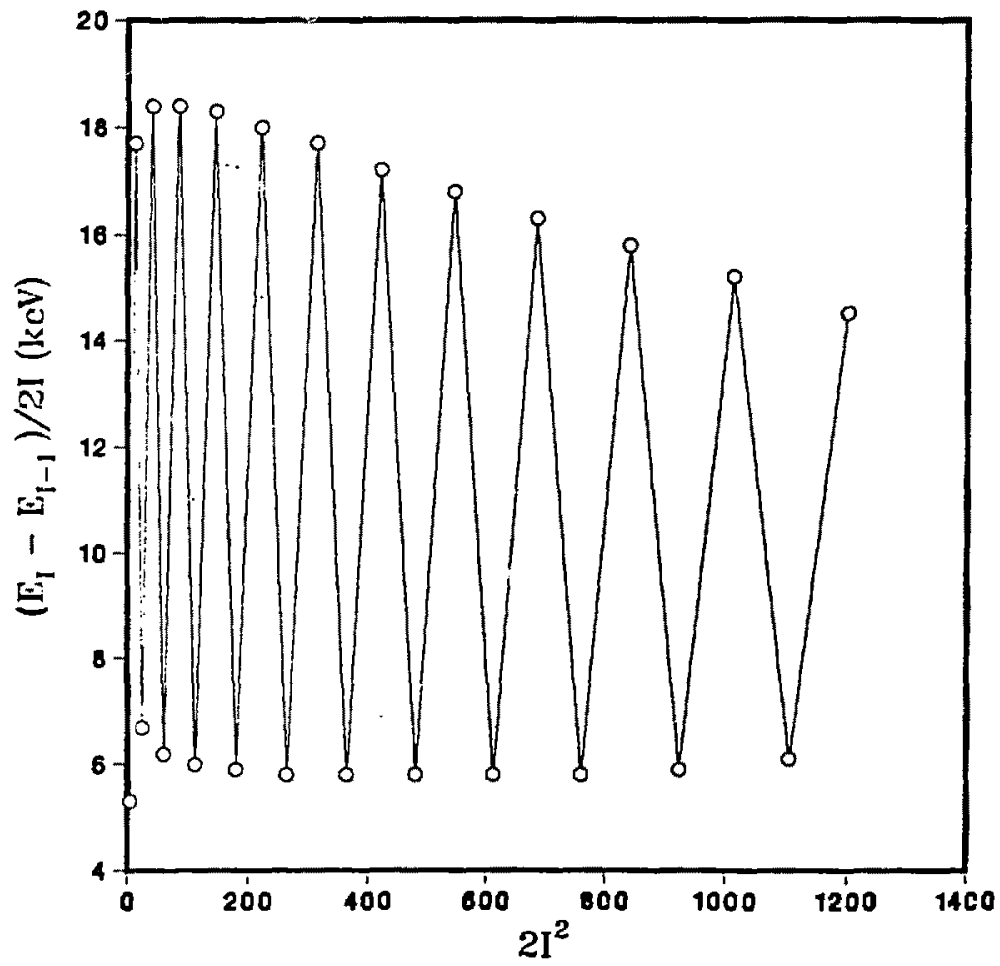

Figure 3.19: Inverse moment-of-inertia, $\Im^{(1)}$, for the ground-state band in 2"Pu showing the magnitude of the staggering between the favoreci and unfavored signatures. 


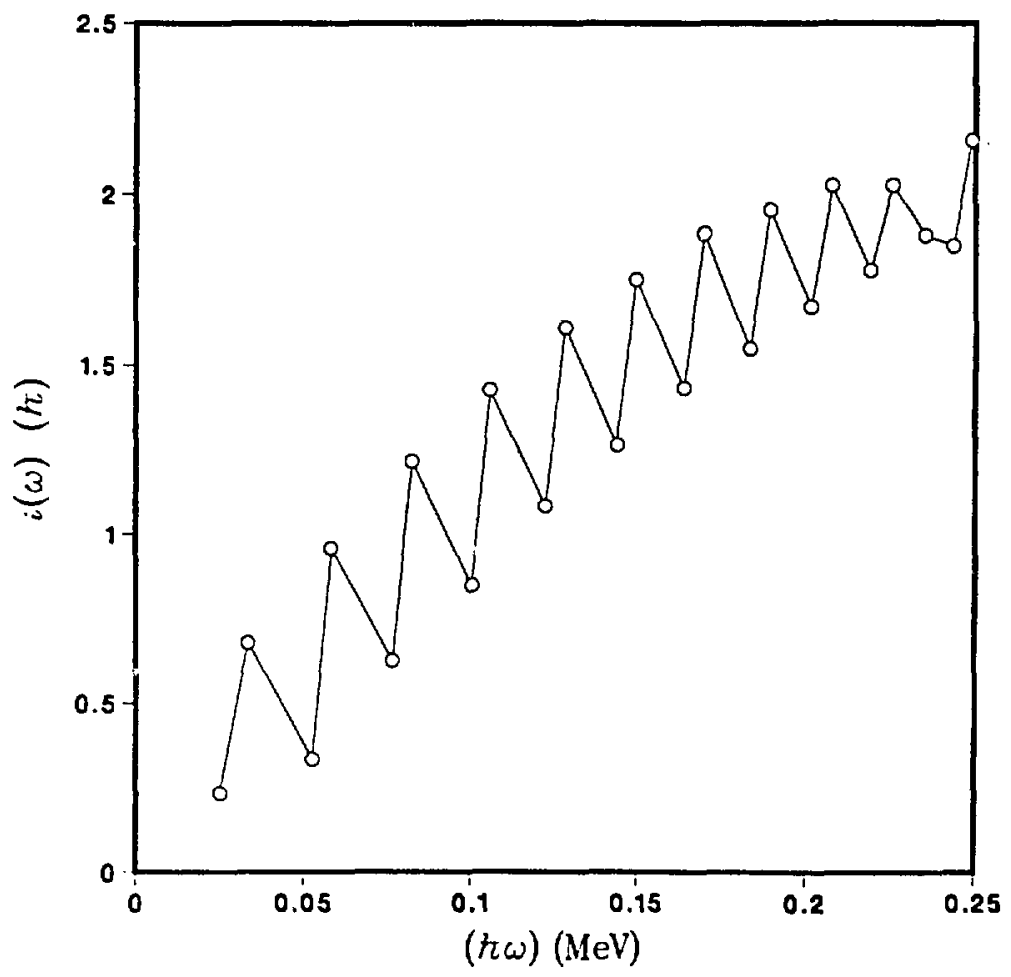

Figure 3.20: Aligned angular momentum $\left(i_{a}\right)$ for the ground-state band in ${ }^{239} \mathrm{Pu}$. Note the anomaly at $\omega \approx 0.24 .{ }^{238} \mathrm{Pu}$ was used as the reference state, with $\Im_{0}=6 \pi \cdot 9 h^{2} / \mathrm{MeV}$ and $\Im_{1}=320 h^{4} / \mathrm{MeV}^{3}$. 
The unfavored signature $(\bullet)$ is above the favored signature (open circles), and the splitting is very large as expected for a $K=\frac{1}{2}^{+}$band. The Routhians decrease smoothly with increasing $\omega$. The decrease is very similar to that for ${ }^{233} \mathrm{U}$; however, from the inverse moment-of-inertia plot, there is no : dication of a reversal of staggering in this nucleus.

A cranking calculation for ${ }^{239} \mathrm{Pu}$ was performed, and the $\frac{1}{2}^{+}[631] \mathrm{g}$. oundstate band shows the expected behavior even with no $\gamma$-deformation (see Figure 3.22). The lowest level is the $\frac{1}{2}^{+}$[631] ground state, with the first exc: :ed level the $\frac{5}{2}^{+}[622]$ and the second excited level the $\frac{7}{2}^{+}$[624]. In addition, it higher spins, there seems to be a band interaction between the $\frac{1}{2}^{+}[631]$ and $\frac{5}{2}^{+}[622]$ bands which could explain the observed anomalies in the momentsof-inertia. Also, the $\frac{5}{2}^{+}$and the $\frac{7}{2}^{+}$bands show a strong interaction arousd $\hbar \omega / \hbar \omega_{0} \approx 0.018$. These bands have a first order Coriolis coupling so this is not surprising. The band mixing in this region is large. The results depend very sensitively on the location of the Fermi surface (or identically the choice of the chemical potential, $\lambda$ ). The conclusion, then, is that ${ }^{239} \mathrm{Pu}$ at high spins may not have a triaxial nuclear shape, or even a very small amount of triaxiality, certainly much less than observed for ${ }^{233} \mathrm{U}$ in Section 3.2 .

Figure 3.23 presents the inelastic $(H, K)$ spectrum gated on the 201.1- 


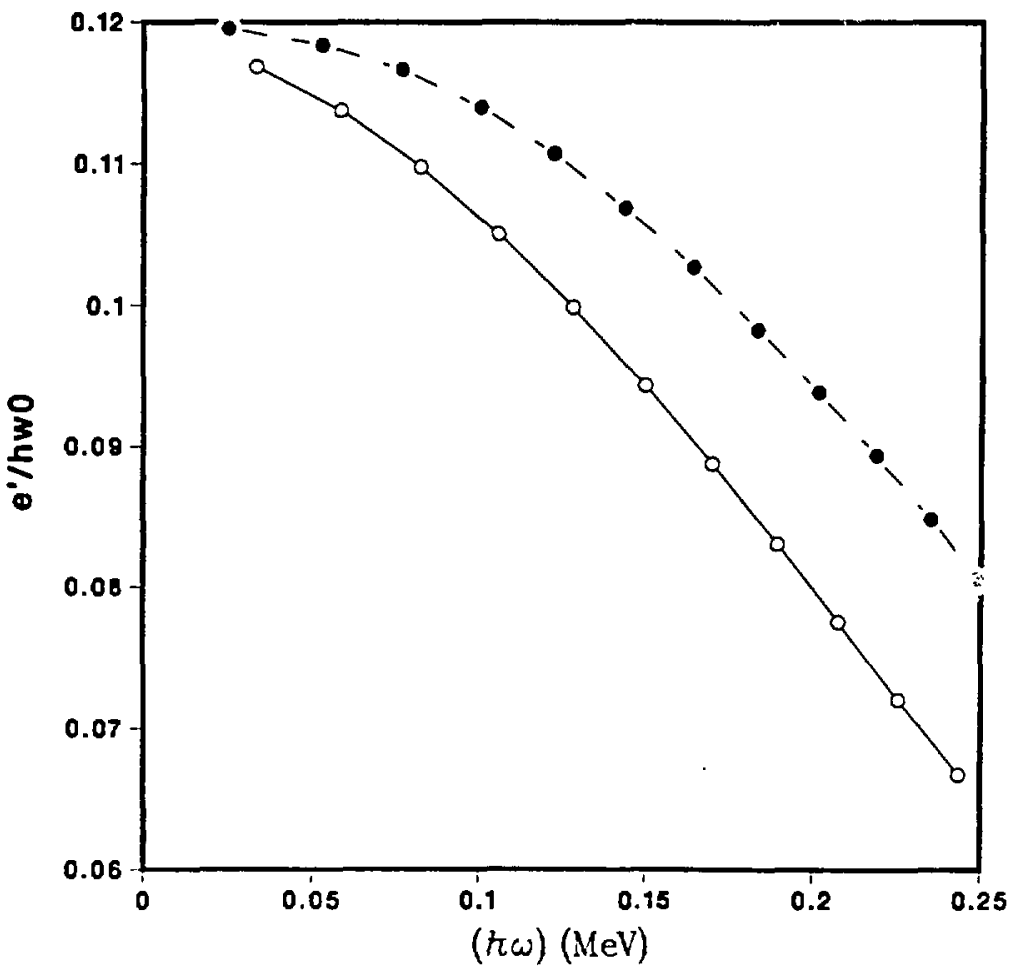

Figure 3.21: Experimental Routhians for the ground-state band in ${ }^{239} \mathrm{Pu}$. The favored (open circles) and the unfavored signature $(\bullet)$ are plotted separately. Note that the splitting between sigratures is large as would be expected for a $K=\frac{1}{2}$ band. 


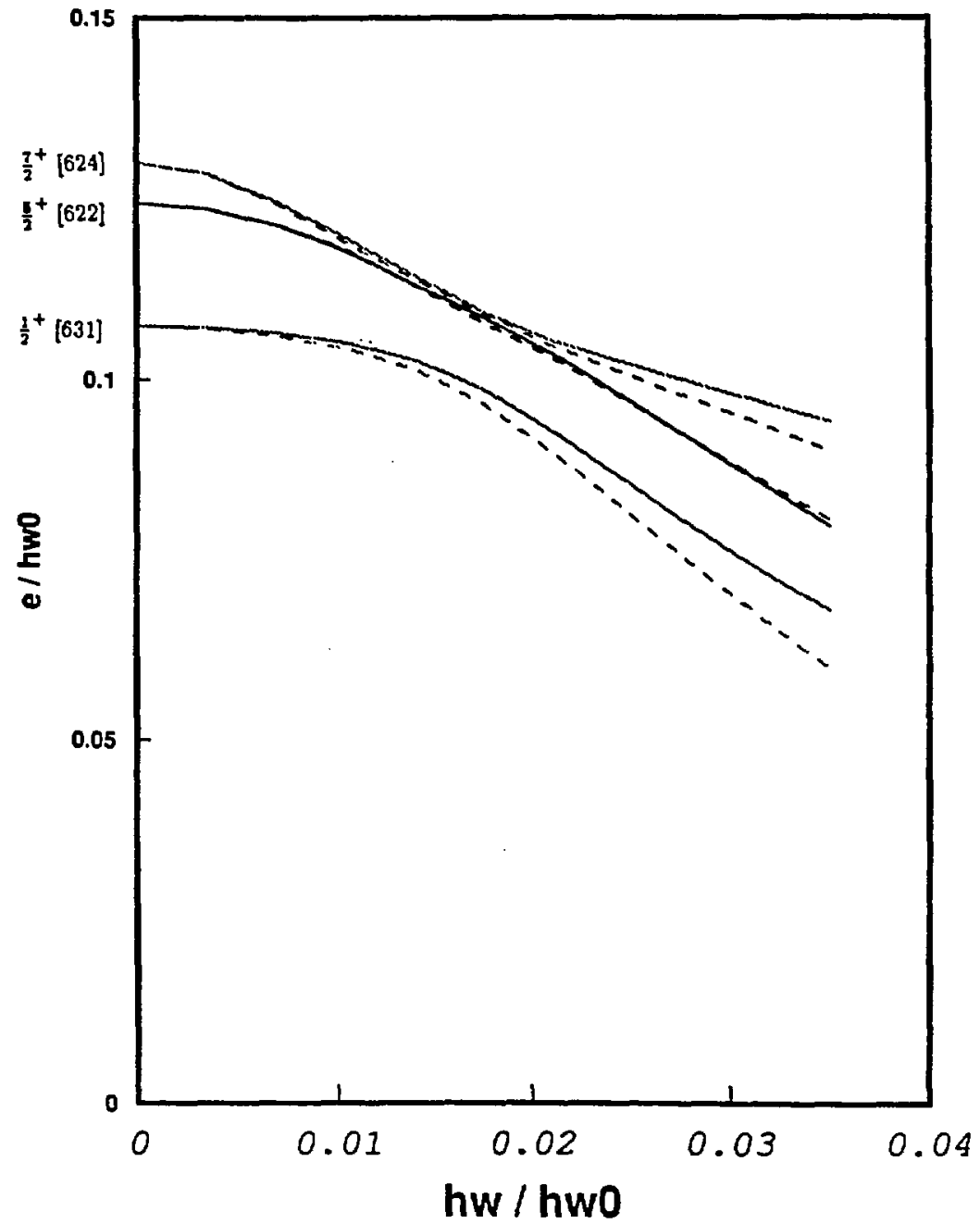

Figure 3.22: Theoretical Routhians for ${ }^{239} \mathrm{Pu}$ as a function of $\omega$. The first level is the $\frac{1}{2}^{+}[631]$ ground state. The first excited level is the $\frac{5}{2}^{+}[622]$ and the second excited state is the $\frac{7}{2}^{+}[624]$. The solid lines are the unfavored signatures and the dashed lines are the favored signatures. 
$\mathrm{keV} \frac{17}{2}^{+} \rightarrow \frac{13}{2}^{+}, 212.1-\mathrm{keV} \frac{19}{2}^{+} \rightarrow \frac{15}{2}^{+}, 288.3-\mathrm{keV} \frac{25}{2}^{+} \rightarrow \frac{21}{2}^{+}$and $299.7-\mathrm{keV}$ $\frac{27}{2}^{+} \rightarrow \frac{23}{2}^{+}$transitions in the Ge detectors, and Figure 3.24 shows the $H$ and $K$ projections. The average multiplicity, $K$, is 3 and the energy, $H$, is $\sim 600 \mathrm{keV}$, centered at a typically low $(H, K)$ for an inelastic reaction. However, as can be seen in comparison to the analogous transfer $(H, K)$ spectrum shown in Figures 3.28 and 3.29 , there is very little difference between the inelastic and transfer multiplicities and total reaction energies. Generally, transfer multiplicities and energies tend to have higher $\left(H, K^{\prime}\right)$ peaks associated with the formation of two quasi-particle states when a pair of neutrons below the Fermi surface is broken. This will be discussed more completely in Section 3.3.2. Rotational population patterns for the ground-state band in ${ }^{239} \mathrm{Pu}$ are presented and discussed in Chapter 6.

\subsection{2 ${ }^{238} \mathrm{Pu}$}

Figure 3.25 shows the Ge $\gamma$-ray spectrum gated on three known ${ }^{238} \mathrm{Pu}$ transitions, the $307-\mathrm{keV} 12^{+} \rightarrow 10^{+}, 349-\mathrm{keV} 14^{+} \rightarrow 12^{+}$and $389-\mathrm{keV} 16^{+} \rightarrow 14^{+}$ transitions. Lower transitions are masked by the inelastic ${ }^{239} \mathrm{Pu} \gamma$-rays. The $6^{+} \rightarrow 4^{+} 158-\mathrm{keV}^{238} \mathrm{Pu}$ ๆ-ray appears as a shoulder on the $15 \cdot 4 \mathrm{keV} \frac{13}{2}^{+} \rightarrow \frac{9}{2}^{+}$ 




Figure 3.23: Inclastic $(I, K)$ spectrum for the ${ }^{90} \mathrm{Zr}+{ }^{239} \mathrm{Pu}$ reaction. 



Figure 3.24: Projections of a) $H$ and b) $K$ for the ${ }^{90} \mathrm{Zr}+{ }^{239} \mathrm{Pu}$ reaction. 


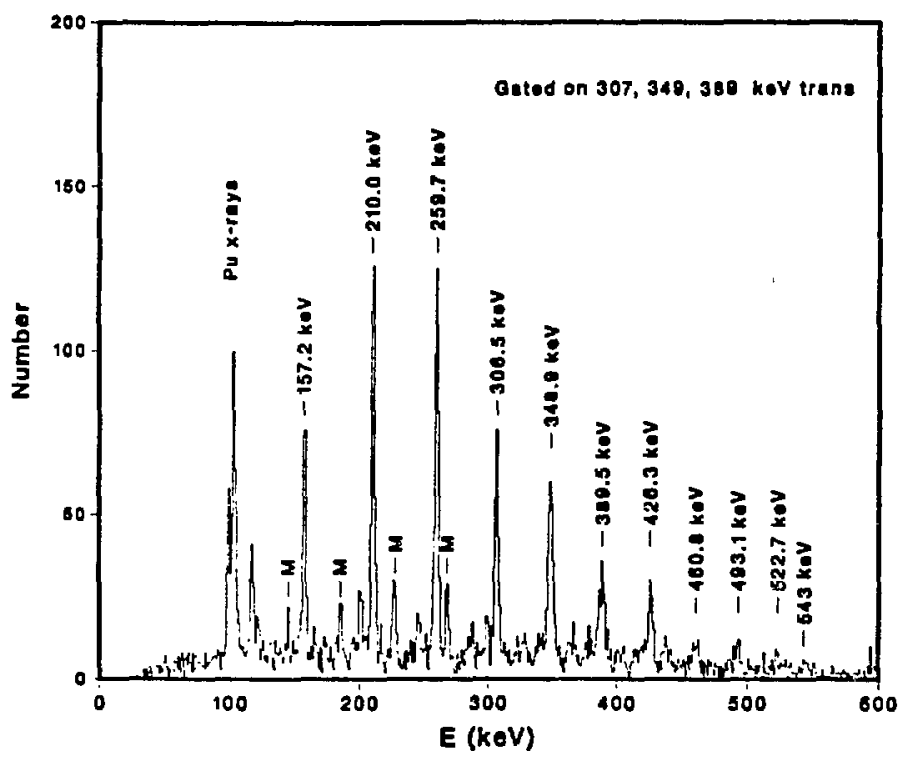

Figure 3.25: The $\gamma$-ray spectrum gated on three known ${ }^{238} \mathrm{Pu}$ transitions, the $307-\mathrm{keV} 12^{+} \rightarrow 10^{+}, 349-\mathrm{keV} 14^{+} \rightarrow 12^{+}$and $389-\mathrm{keV} 16^{+} \rightarrow 14^{+}$transitions for the one-neutron transfer reaction. The ${ }^{238} \mathrm{Fu}$ transitions are marked with energies. The "mystery" band discussed in the text is labelled with M's. 
${ }^{239} \mathrm{Pu}$ inelastic transition, too close for $\gamma-\gamma$ gating techniques, but sufficiently separated for obtaining intensity information for the transfer reaction. The ground-state rotational band in ${ }^{238} \mathrm{Pu}$ is observed up to the $543-\mathrm{keV} 26^{+} \rightarrow 24^{+}$ transition (there is a candidate for the $28^{+} \rightarrow 26^{+}$transition if one has a little imagination). This is $10 \mathrm{~h}$ higher than observed in the $(\alpha, 4 n)$ work of Hardt et al. [41]. The transition energies shown in Table 3.7 are again very regular. The expected backbending [3S] at about spin $30 \hbar$ was not observed. The data

Table 3. 7 : Observed transitions in ${ }^{238} \mathrm{Pu}$

\begin{tabular}{|c|c|}
\hline Transition & Energy (keV) \\
\hline $6^{+} \rightarrow 4^{+}$ & $157.2(2)$ \\
$8^{+} \rightarrow 6^{+}$ & $210.0(2)$ \\
$10^{+} \rightarrow 8^{+}$ & $259.7(2)$ \\
$12^{+} \rightarrow 10^{+}$ & $306.5(2)$ \\
$14^{+} \rightarrow 12^{+}$ & $348.9(2)$ \\
$16^{+} \rightarrow 14^{+}$ & $389.5(2)$ \\
$18^{+} \rightarrow 16^{+}$ & $426.3(2)$ \\
$20^{+} \rightarrow 18^{+}$ & $460.8(2)$ \\
$22^{+} \rightarrow 20^{+}$ & $493.1(2)$ \\
$24^{+} \rightarrow 22^{+}$ & $522.7(2)$ \\
$26^{+} \rightarrow 24^{+}$ & $544.4(2)$ \\
\hline
\end{tabular}

do not rule out a backbend at higher spins.

The moments-of-inertia for the ground-state band in ${ }^{238} \mathrm{Pu}$ are presented in Figure 3.26. The kinematic moment-of-inertia is extremely regular, and 


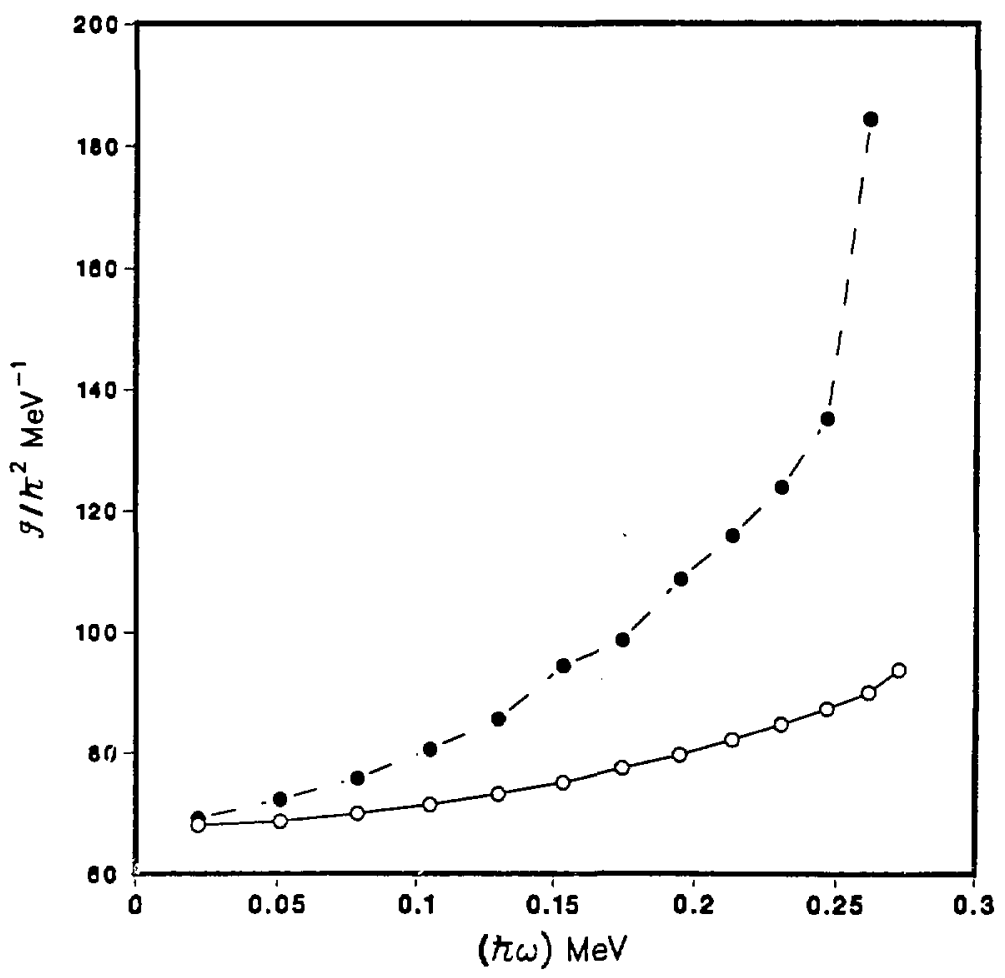

Figure 3.26: Kinematic (open circles) and dynamic ( $\bullet$ moments-of-inertia for the ground-state band in ${ }^{238} \mathrm{Pu}$. 
only slightly increases with increasing spin, indicating that the deformation is constant with increasing spin. However, the dynamic moment-of-inertia, a much more sensitive probe, exhibits a large increase at higher spins. In addition, the $26^{+}$level has an anomalously high $\Im^{(2)}$ (and $\Im^{(1)}$ is slightly higher than normal), perhaps the first indication of a backbend in this nucleus.

Single particle energy level Routhians, $e^{\prime}$, are again extracted, as in Section 3.2, and are shown in Figure 3.27. The reference state was chosen to be ${ }^{238} \mathrm{Pu}$ using $\Im_{0}$ and $\Im_{1}$ fit using the lowest five transitions. Thus, the extracted Routhians are expected to yield a horizontal line with slope $=0$ (what all Routhians would be if the correct reference state were chosen). Indeed, the extracted Routhians only vary by $0.002 / \hbar \omega_{0}(\sim 20 \%)$ over the range of spins observed, whereas the Routhians for ${ }^{239} \mathrm{Pu}$ varied by a factor of two. The Routhians decrease with increasing spin, and the highest spin is anomalously low. Indced, in tine region just above the levels used to obtain $\Im_{0}$ and $\Im_{1}$, the Routhians are constant to \pm 0.00001 . It is only the $26^{+}$level which deviates by a factor of 70 . Of course, single particle energy levels have relatively little meaning in a nucleus consisting entirely of pairs of particles; however, this exercise does provide an additional clue that band mixing at the highest spins observed in ${ }^{238} \mathrm{Pu}$ may be occurring. No cranking calculation was performed 


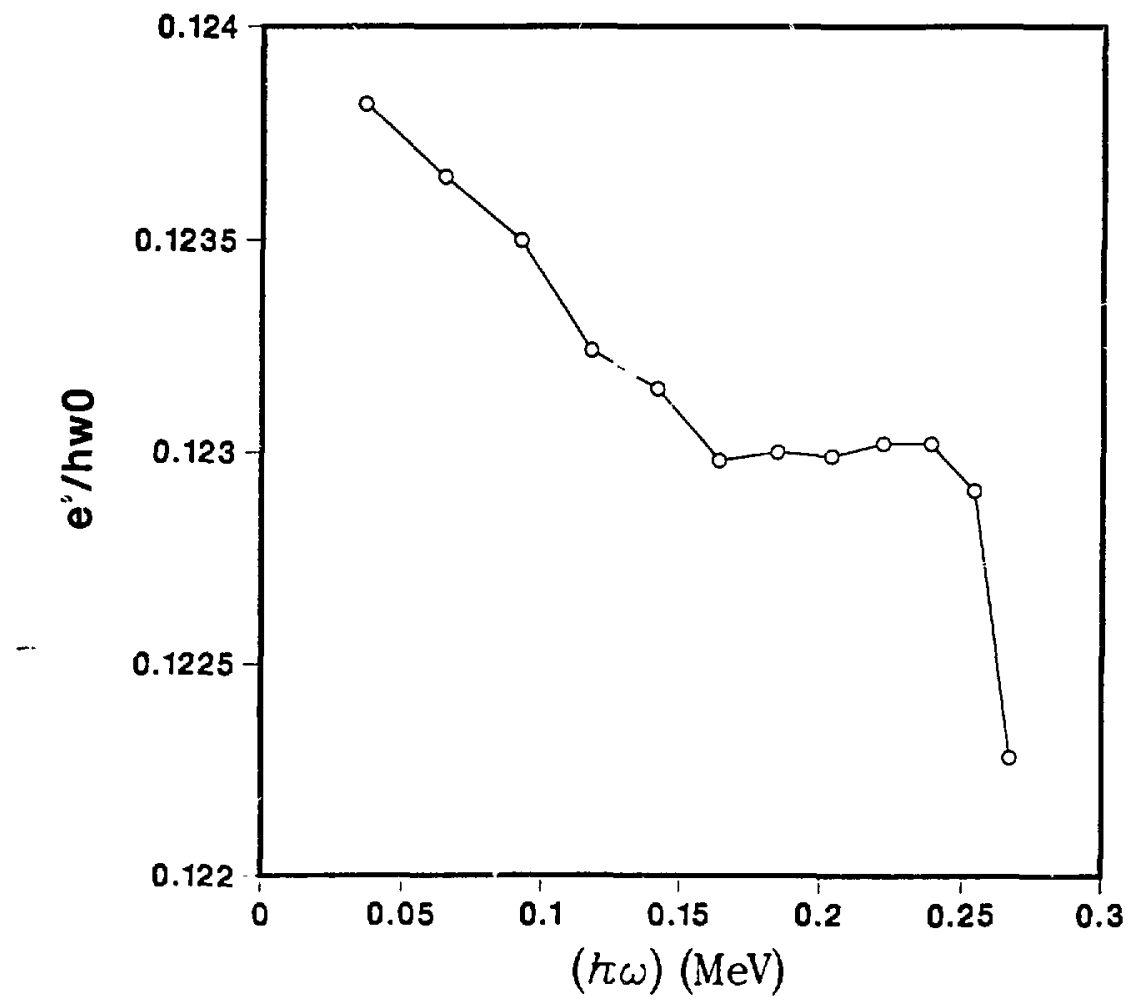

Figure 3.27: Experimental Routhians for ${ }^{238} \mathrm{Pu}$. 
as results analogous to ${ }^{239} \mathrm{Pu}$ are expected.

Figures 3.28 and 3.29 show the transfer $(H, K)$ spectrum, and the $H$ and $K^{*}$ projections gated on. the $30 \mathrm{~T} \cdot \mathrm{keV} 12^{+} \rightarrow 10^{+}, 349-\mathrm{keV} 14^{+} \rightarrow 12^{+}$and 389-keV $16^{+} \rightarrow 14^{+}{ }^{238} \mathrm{Pu}$ transitions detected in the Ge detectors. The average multiplicity for the transfer reaction is $3-4$ and the average total reaction energy is $800 \mathrm{keV}$, both slightly higher than the inelastic reaction. No second maximum for two quasi-particle transfer is observed. The cross-section for the two quasi-particle one-neutron transfer reaction is either suppressed, or transfer of the $K=\frac{1}{2}^{+}$neutron is enhanced in this reaction. It is possible that the $K=\frac{1}{2}$ neutron satisfies any Brinks selection rules much more easily than the neu :rons below the Fermi surface in the $N=7, \frac{7}{2}^{-}[743]$ or $N=5, \frac{1}{2}^{-}[501]$ intruder levels. Additionally, the beam energy may have been coincidentally selected so as to enhance the ground-ground transfer and not the two quasiparticle transfer, as the cost to break a pair of neutrons (pairing energy) might be tow high under these particular reaction conditions. This result is rather surprising, as it is the first observation of such a phenomenon; however, it is also the first $K=\frac{1}{2}$ neutron transfer studied by our collaboration. 


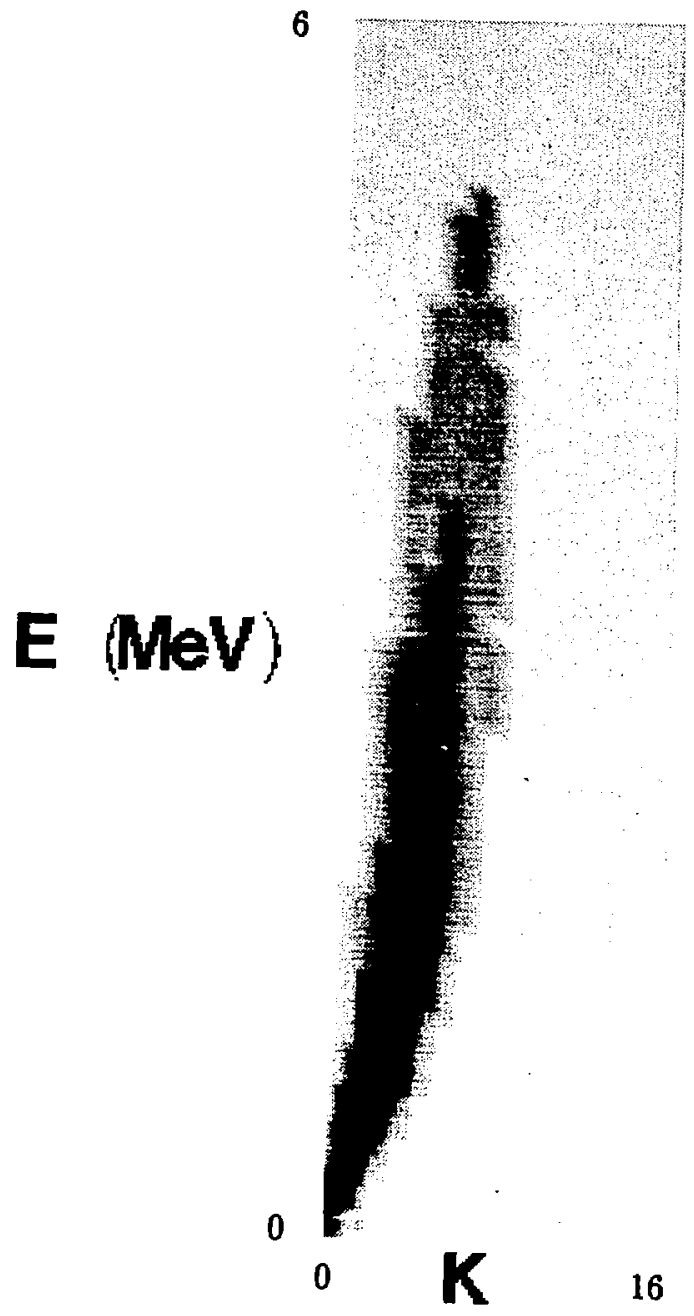

Figure 3.28: Transfer $(H, K)$ spectrum for ${ }^{238} \mathrm{Pu}$. 

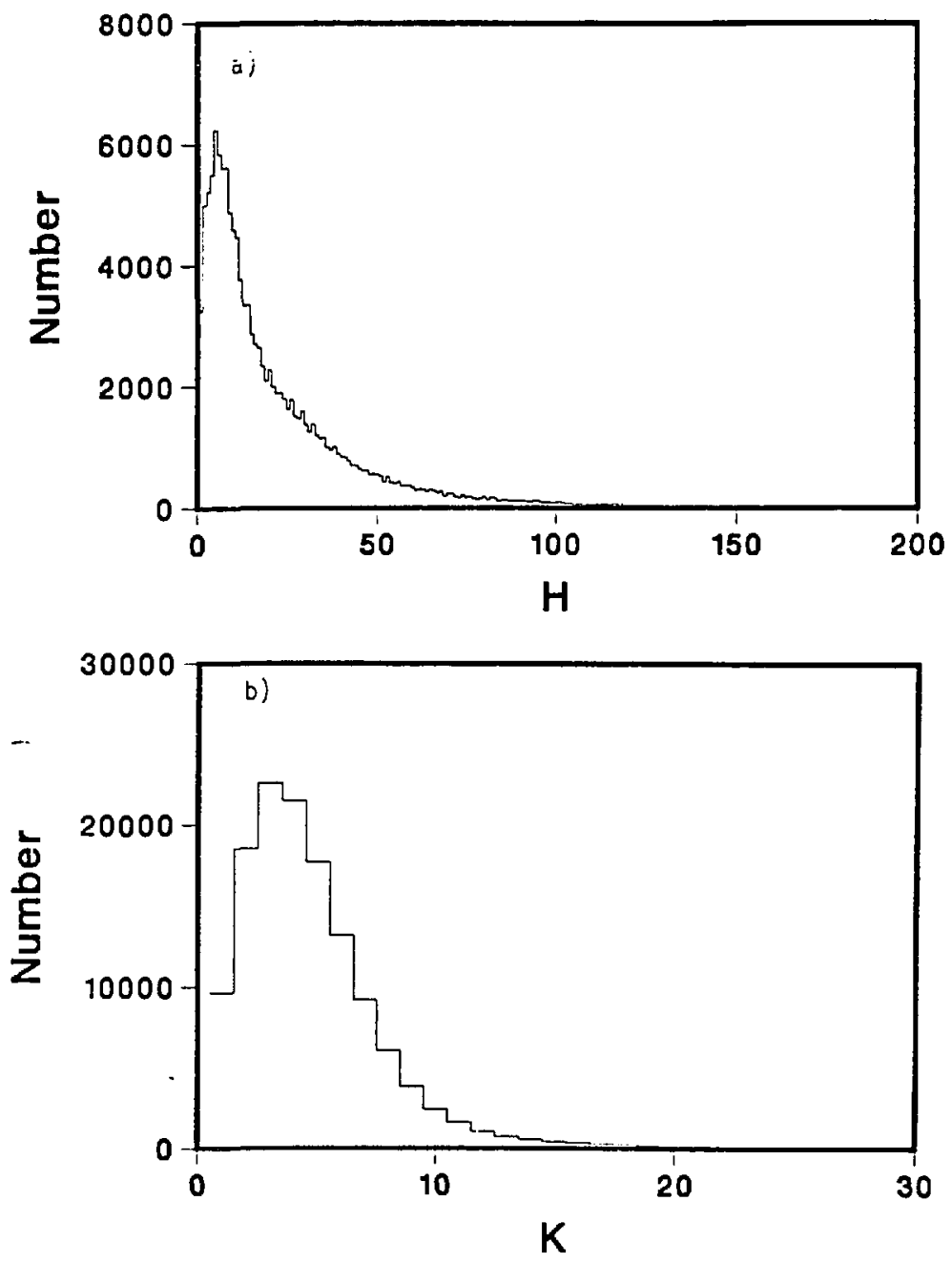

Figure 3.29: Projections of aj $H$ and b) $K^{\prime}$ for the transfer reaction. 
The differential cross-section is given by:

$$
\frac{d \sigma(\theta)}{d \Omega}=\left(\frac{d \sigma(\theta)}{d \Omega}\right)_{\text {elentic }} P_{I}
$$

and

$$
\left(\frac{d \sigma(\theta)}{d \Omega}\right)_{\text {elastic }}=\frac{\left(A_{t} Z_{p} Z_{t} e^{2}\right)^{2} 931.5 \csc ^{4}\left(\frac{\theta_{c M}}{2}\right)}{8(197.3)^{2} A_{p} E_{\text {beam }}} .
$$

The $6^{+} \rightarrow 4^{+}{ }^{238} \mathrm{Pu}$ transition in the Ge detectors was fit as a function of scattering angle (see Appendix B for a discussion of the spectrum fitting). The $\gamma$-ray yield is then given by:

$$
Y=\frac{I_{\gamma}(1+\alpha)}{\epsilon N_{\text {sing }}}
$$

where $\alpha$ is the internal conversion coefficient (summed over all energetically allowed shells) for the particular $\gamma$-ray, $\epsilon$ is the total Ge detector efficiency and $N_{\text {sing }}$ is the number of singles. The probability is:

$$
P_{I}=\left(Y_{I}-Y_{S+1}\right) \sin \left(\frac{\theta_{C M}}{2}\right)
$$

Probabilities as a function of spin for the ${ }^{238} \mathrm{Pu}$ ground-state band are presented in Chapter 6.

If one assumes that one-neutron transfer can be approximated by classical Rutherford scattering, the distance of closest approach as a function of 
scattering angle is:

$$
D=\frac{Z_{p} Z_{t} e^{2}\left(1+\left(\sin \theta_{C M}\right)^{-1}\right)}{2 E_{C M}}
$$

and the reduced distance of closest approach, $d_{0}$, is given by:

$$
d_{0}=\frac{D}{A_{t}^{\frac{1}{3}}+A_{p}^{\frac{1}{3}}} .
$$

The impact parameter, $b$, is given by the usual equation:

$$
b=\frac{Z_{p} Z_{i} e^{2}}{\mu v_{r e l}^{2}} \tan \theta_{C M}
$$

The differential cross-section for one-neutron transfer at $\theta_{\text {graze }}=140^{\circ}$ in the lab is $\sim 80 \mathrm{mb} / \mathrm{sr}$, assuming all de-excitation flows through the $6^{+}$level (based on the $6^{+} \rightarrow 4^{+}$intensity fits). The differential cross-sections as a function of distance of closest approach, $D$, and reduced distance of closest approach, $d_{0}$, for lab and center-of-mass reference frames are presented in Figures 3.30 and 3.31. The reduced differential cross-sections are shown in Figure 3.32. No abnormalities are observed. However, it should be noted that the cross-section at the grazing angle is a factor of four larger than that for the ${ }^{58} \mathrm{Ni}\left({ }^{235} \mathrm{U},{ }^{234} \mathrm{U}\right)^{59} \mathrm{Ni}$ reaction. Although these one-neutron transfer reactions were performed with different projectiles and different beam energies, a comparison at the grazing angle may provide some insight into the reaction mechanism. The distance of 


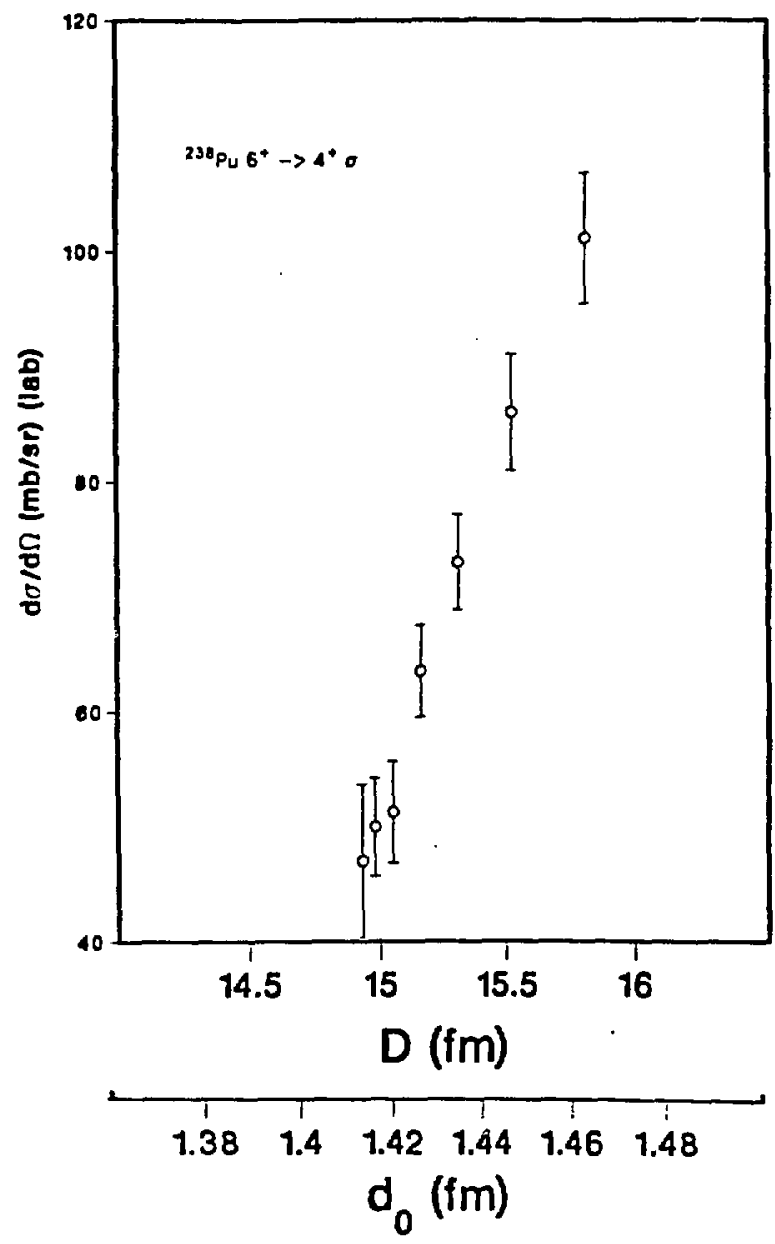

Figure 3.30: Differential cross-sections for one-neutron transfer as a function of distance of closest approach in the lab. 


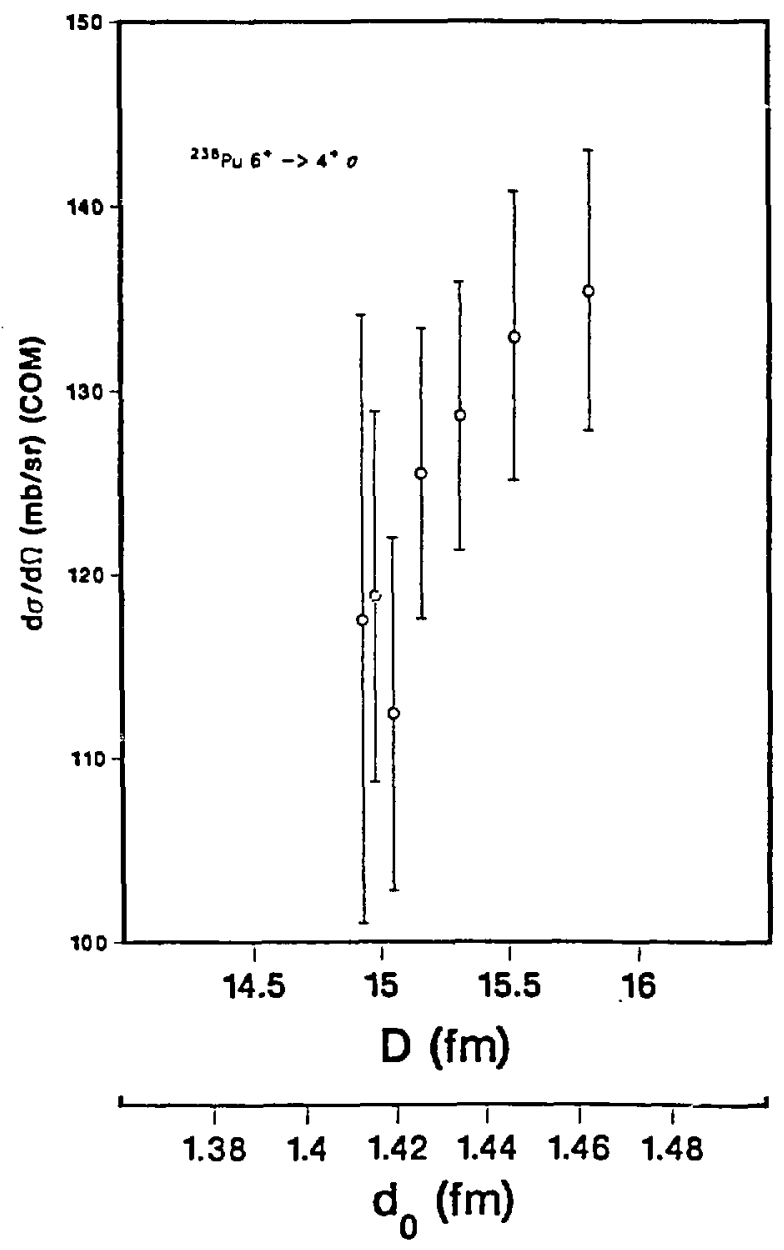

Figure 3.31: Differential cross-sections for one-neutron transfer as a function of distance of closest approach in the center-of-mass frame. 


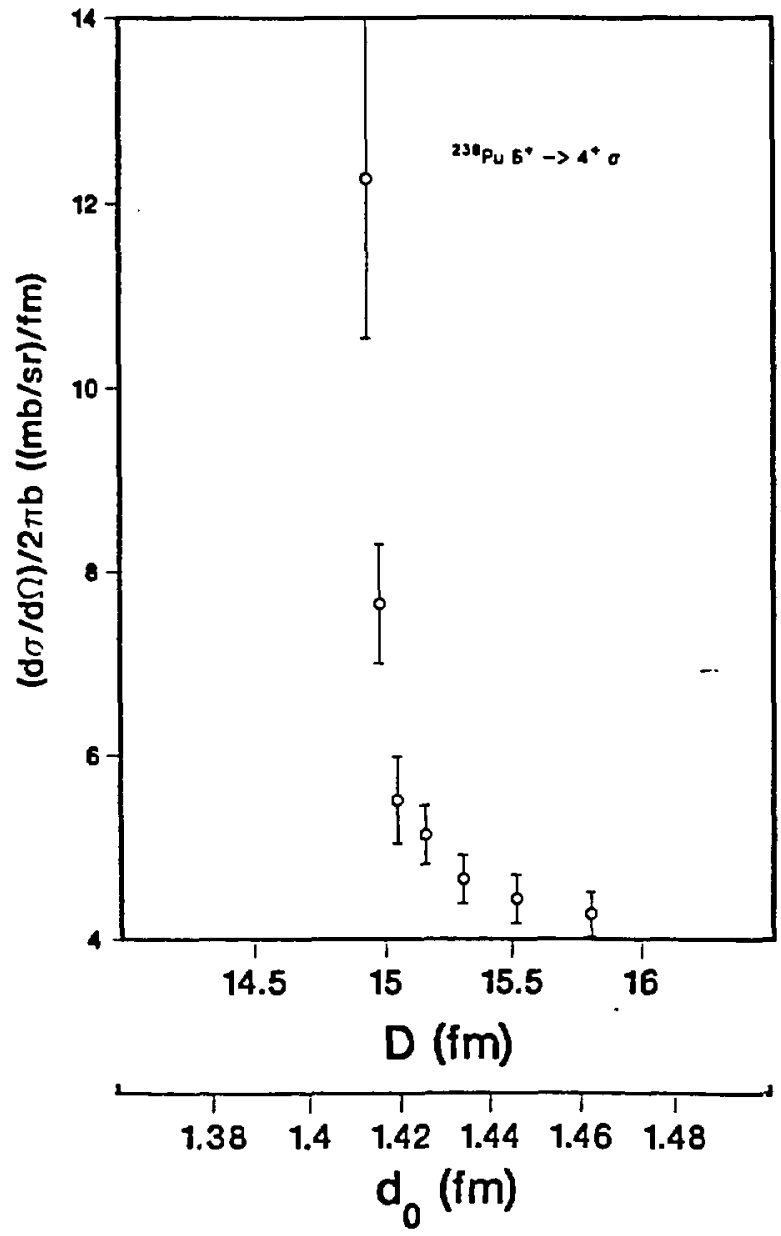

Figure 3.32: Reduced differential cross-sections for one-neutron transfer as a function of distance of closest approach in the lab. 
closest approach is the same for both systems at the grazing angle $(2.25 \mathrm{fm}$ for ${ }^{58} \mathrm{Ni}+{ }^{235} \mathrm{U}$ and $2.27 \mathrm{fm}$ for ${ }^{90} \mathrm{Zr}+{ }^{239} \mathrm{Pu}$ ). However, the Nilsson orbitals involved in the transfer are different (the intruder $\frac{7}{2}^{-}[743]$ for ${ }^{235} \mathrm{U}$ ground-state and the $\frac{1}{2}^{+}[631]$ for ${ }^{239} \mathrm{Pu}$ ground-state), implying that nuclear structure or surface form factors may be playing an important role in these reactions. A discussion of surface form factors is included in Chapters 5 and 6 .

The probability for populating the $6^{+}$state, as a function of $D$ and $d_{0}$ is shown in Figure 3.33. The solid line represents a simple barrier penetration theory calculation where

$$
P \propto \exp \left\{-\frac{\sqrt{2(931.5) E_{\text {bind }}}}{\hbar} R\right\}
$$

and $E_{b i n d}=3.412 \mathrm{MeV}$ for the $K=\frac{1}{2}$ neutron in ${ }^{239} \mathrm{Pu}$ and $R=D-R_{p}-R_{t}$, where $R_{p}$ and $R_{t}$ are the normal radii of the projectile and target, respectively, calculated according to the standard equation $R=r_{0} A^{\frac{1}{3}}$, with $r_{0}=1.25 \mathrm{fm}$. The curve is normalized to the probability at the smallest $D$. Figure 3.33 shows excellent agreement between the data and the simple theory, indicating this one-neutron transfer reaction is arcurately described by simple Breit-Ebe] theory [71]. It would be interesting to extract $P_{2 n}$ for the ${ }^{240} \mathrm{Pu}\left({ }^{90} \mathrm{Zr},{ }^{92} \mathrm{Zr}\right)$ ${ }^{238} \mathrm{P} u$ rcaction to see if $P_{2 n}(D)=\left(P_{1 n}(D)\right)^{2}$, as simple theory predicts, or if 


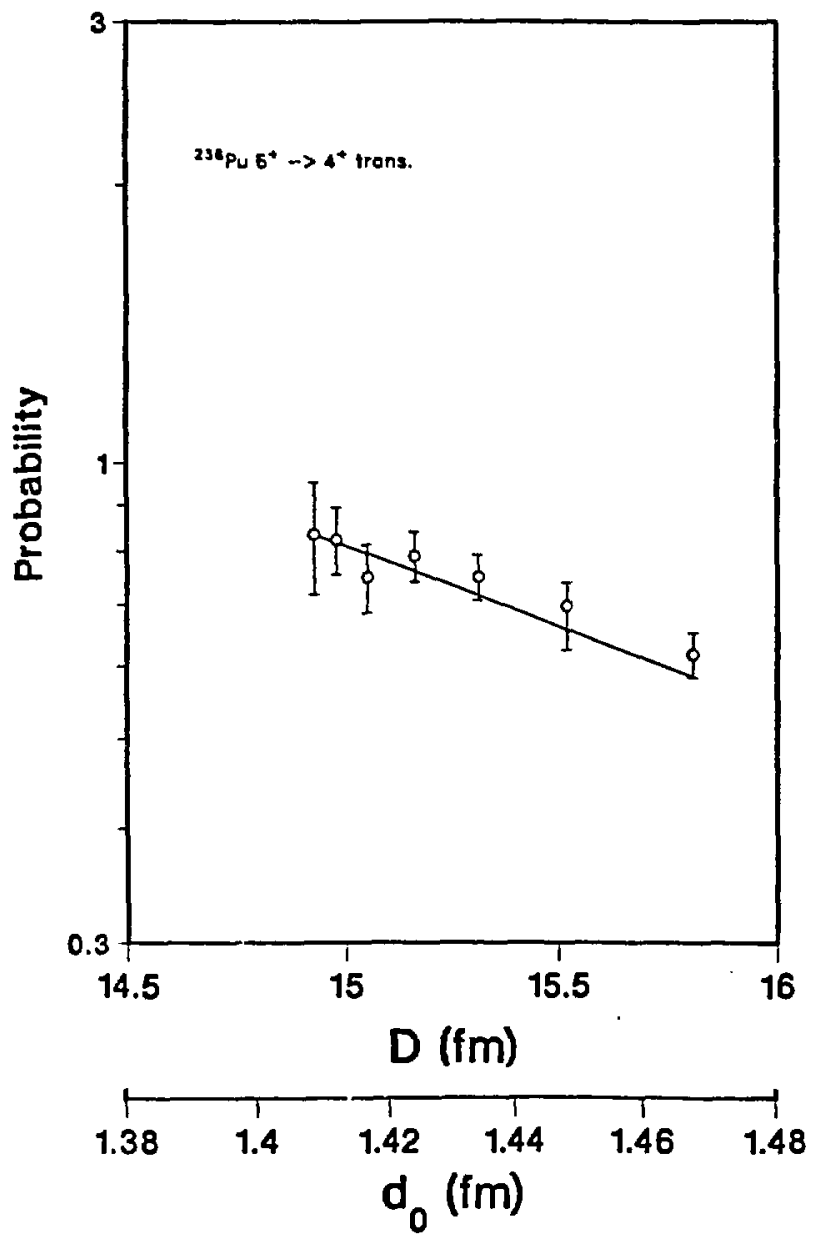

Figure 3.33: One-neutron transfer probability as a function of distance of closest approach. The solid line represents a simple theory based on equation (3.34). 
an enhancement of two-neutron transfer exists, as in reference [72].

In addition to observing ground-state band transitions in ${ }^{238} \mathrm{Pu}$ and ${ }^{239} \mathrm{Pu}$, we observed four "mystery" transitions located at $145 \mathrm{keV}, 185 \mathrm{keV}, 227 \mathrm{keV}$ and $268 \mathrm{keV}$, respectively. These transitions are spaced about $40 \mathrm{keV}$ apart, suspiciously like a rotational band with the same dynamic moment-of-inertia as the ground-state band in ${ }^{238} \mathrm{Pu}$. As can be seen in the Ge $\gamma$-ray spectra gated on ${ }^{238} \mathrm{Pu}$ and ${ }^{239} \mathrm{Pu}$ transitions (see Figures 3.25 and 3.17 ), these transitions appear more strongly in the ${ }^{238} \mathrm{Pu}$ spectrum. These transitions correspond to no known $\gamma$-ray transitions in ${ }^{237} \mathrm{Pu},{ }^{238} \mathrm{Pu},{ }^{239} \mathrm{Pu},{ }^{210} \mathrm{Pu},{ }^{241} \mathrm{Pu}$ and several one-proton transfer products ${ }^{238} \mathrm{~Np}$ and ${ }^{240} \mathrm{Am}$. Also, because the $\gamma$-ray lines appear as sharp, well-defined peaks in the Pu-like Doppler shifted spectrum, this excludes any $\mathrm{Zr}$-like sources (which would have a different Doppler shift), and known sources of background (which wonld be stationary sources in the lab). Thus, individual gates were set on these four transitions to investigate the source of these $\gamma$-rays. Figures $3.34,3.35,3.36$ and 3.37 present the $\gamma-\gamma$ coincidence spectra gated on individual "mystery" transitions including background subtraction.

Figure 3.34 is the $\gamma$-ray spectrum gated on the $145-\mathrm{keV}$ transition. Possible peaks are marked with energies. A clear peak in the spectrum is the $268-\mathrm{keV}$ 


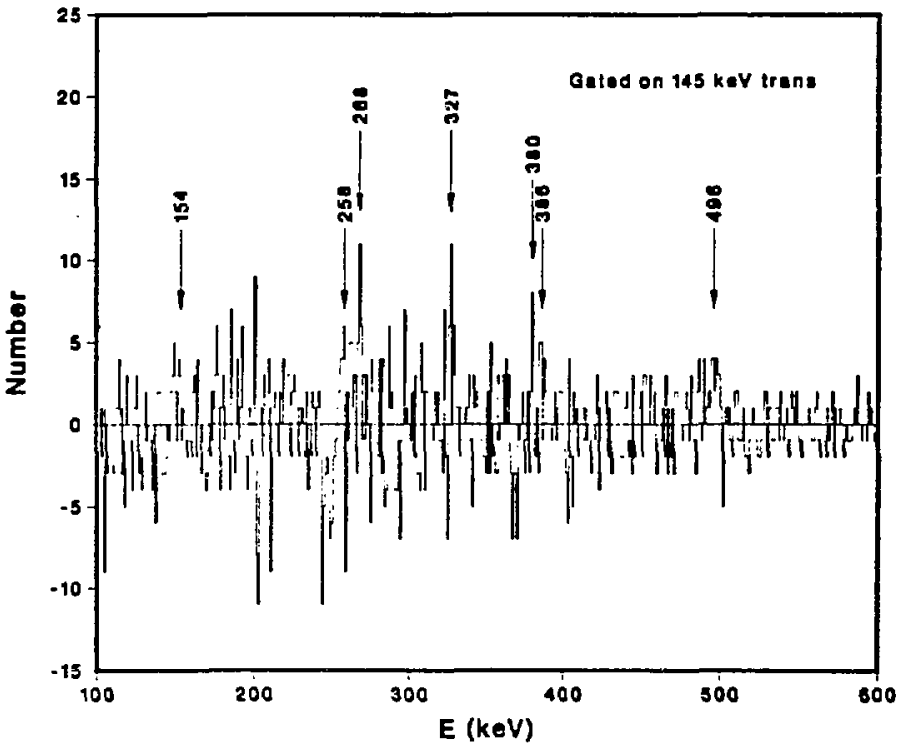

Figure 3.34: Ge $\gamma$-ray spectrum gated on the "mystery" transition at $145 \mathrm{keV}$ in the ${ }^{90} \mathrm{Zr}+{ }^{239} \mathrm{Pu}$ experiment. 


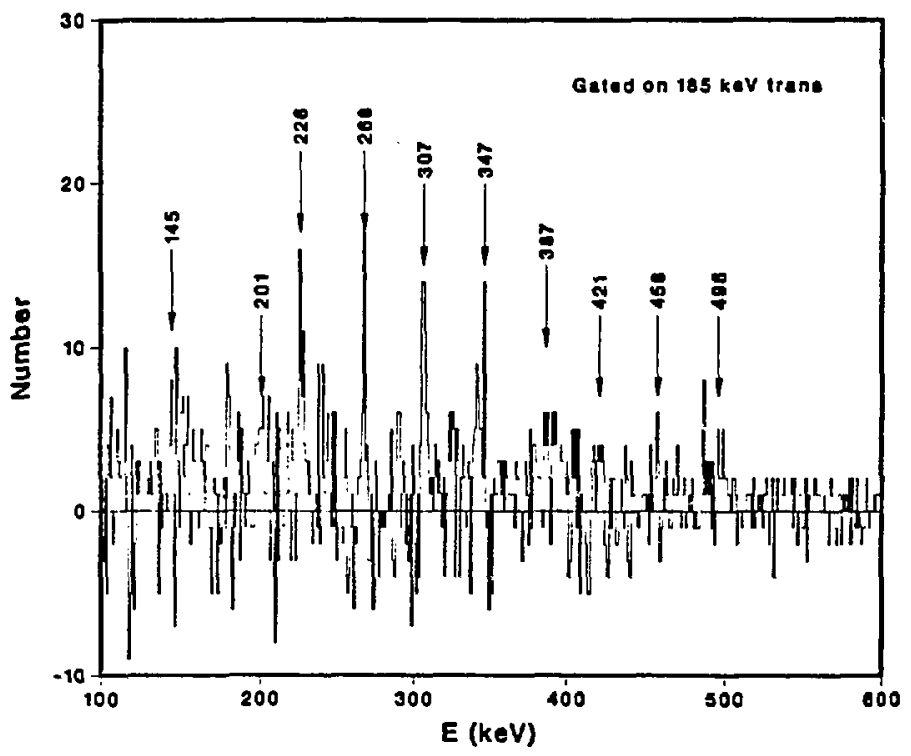

Figure 3.35: Ge $\gamma$-ray spectrum gated on the "mystery" transition at $185 \mathrm{keV}$ in the ${ }^{90} \mathrm{Zr}+{ }^{239} \mathrm{Pu}$ experiment. 


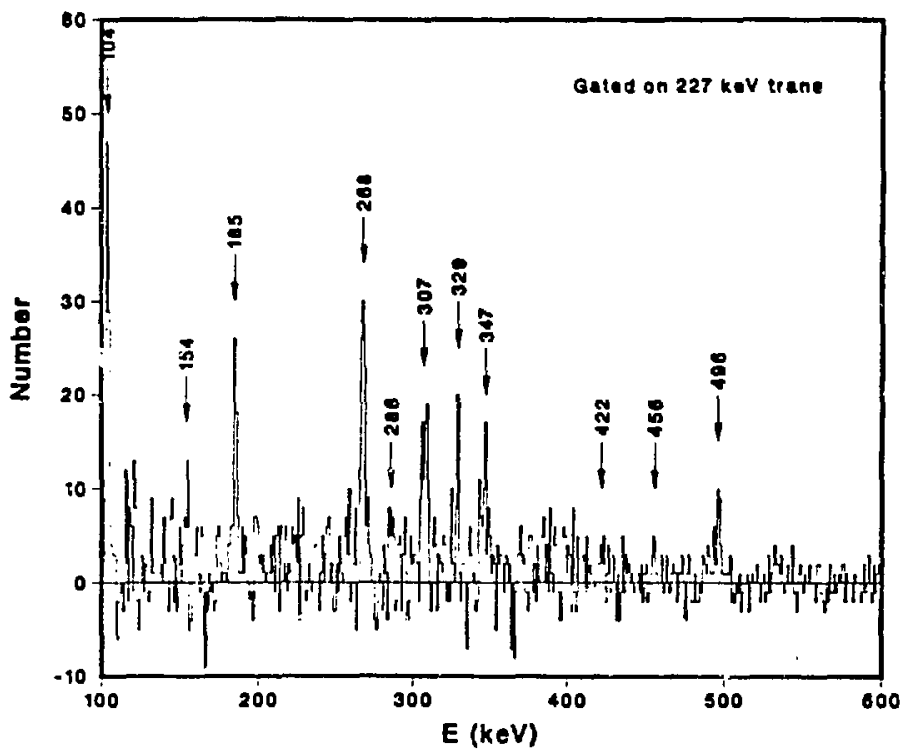

Figure 3.36: Ge $\gamma$-ray spectrum gated on the "mystery" transition at $226 \mathrm{keV}$ in the ${ }^{90} \mathrm{Zr}+{ }^{239} \mathrm{Pu}$ experiment. 


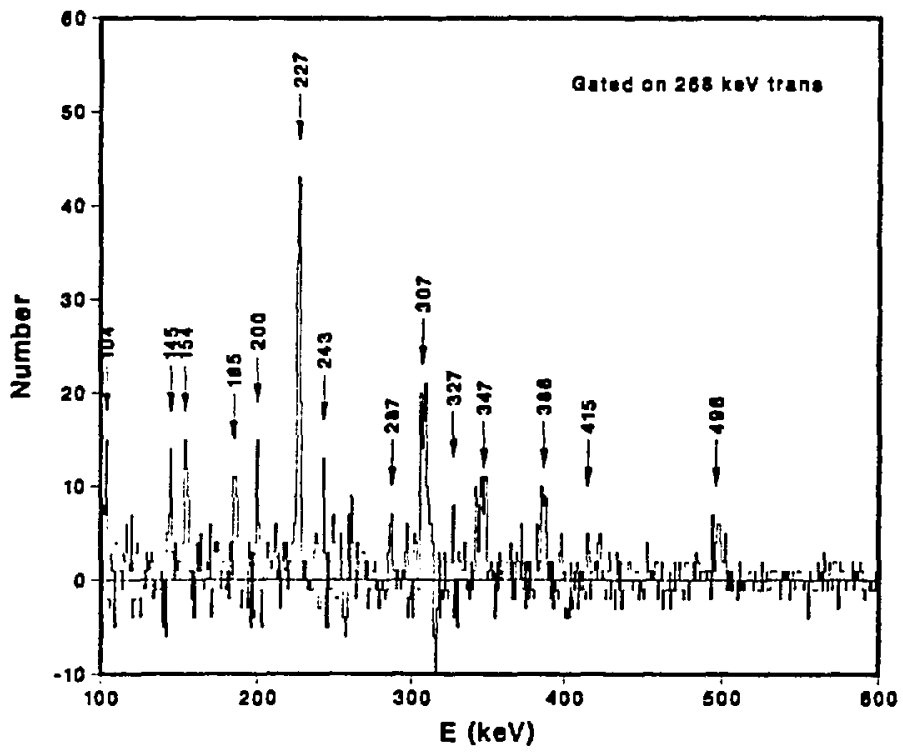

Figure 3.37: Ge $\gamma$-ray spectrum gated on the "mystery" transition at $268 \mathrm{keV}$ in the ${ }^{90} \mathrm{Zr}+{ }^{239} \mathrm{Pu}$ experiment. 
"mystery" transition. Lack of statistics precludes a definitive statement on other $\gamma-\gamma$ coincidences; however, possible peaks at $258-\mathrm{keV}\left(10^{+} \rightarrow 8^{+}{ }^{238} \mathrm{Pu}\right)$, $\left.327-\mathrm{keV} \quad \frac{29}{2}^{+} \rightarrow \frac{25}{2}^{+}{ }^{239} \mathrm{Pu}\right), \quad 380-\mathrm{keV} \quad\left(\frac{35}{2}^{+} \rightarrow \frac{31}{2}^{+}{ }^{239} \mathrm{Pu}\right)$, $386-\mathrm{keV}\left(16^{+} \rightarrow 14^{+}{ }^{238} \mathrm{Pu}\right)$ and $496 \cdot \mathrm{keV}\left(22^{+} \rightarrow 20^{+}{ }^{238} \mathrm{Pu}\right)$ can be identified.

Figure 3.35 is gated on the $185-\mathrm{keV}$ transition. Four clear peaks are observed; the $226-\mathrm{keV}$ and $268-\mathrm{keV}$ "mystery" transitions (the 145-keV "mystery" transition is marginally identifiable), and the $307-\mathrm{keV}\left(12^{+} \rightarrow 10^{+}\right.$ $\left.{ }^{238} \mathrm{Pu}\right)$ and $347-\mathrm{keV}\left(14^{+} \rightarrow 12^{+}{ }^{238} \mathrm{Pu}\right)$ transitions. Four other ${ }^{238} \mathrm{Pu}$ transitions are partially identifiable $\left(387-\mathrm{keV} 16^{+} \rightarrow 14^{+}, 421-\mathrm{keV} 18^{+} \rightarrow 16^{+}\right.$, $458-\mathrm{keV} 20^{+} \rightarrow 18^{+}$and $497 \cdot \mathrm{keV} 22^{+} \rightarrow 20^{+}$). Also, the $201 \cdot \mathrm{keV} \frac{17}{2}^{+} \rightarrow \frac{13}{2}^{+}$ ${ }^{239} \mathrm{Pu}$ transition is seen (perhaps just background). This spectrum clearly indicates that the four "mystery" transitions are indeed in coincidence, and, furthermore, that the $185-\mathrm{keV}$ transition is in coincidence with ${ }^{238} \mathrm{Pu}$ transitions, implying that the "mystery" band belongs to ${ }^{239} \mathrm{Pu}$.

However, the picture becomes less clear upon examination of Figure 3.36 , gated on the $22 \pi-k e V$ transition. Seven clear peaks are seen. The peak located at $104 \mathrm{keV}$ is identified with the $\mathrm{Pu} x$-rays. In addition, the $185-\mathrm{keV}$ and 268keV "mystery" transitions are observed. Three remaining peaks are assigned 
to ${ }^{238} \mathrm{Pu}\left(308-\mathrm{keV} 12^{+} \rightarrow 10^{+}, 347-\mathrm{keV} 14^{+} \rightarrow 12^{+}\right.$and $\left.496-\mathrm{keV} 22^{+} \rightarrow 20^{+}\right)$ and one to ${ }^{239} \mathrm{Pu}\left(329-\mathrm{keV} \frac{29}{2}^{+} \rightarrow \frac{25}{2}^{+}\right)$. There are also candidate peaks for $422-\mathrm{keV} 18^{+} \rightarrow 16^{+}{ }^{239} \mathrm{Pu}, 456-\mathrm{keV} 20^{+} \rightarrow 18^{+238} \mathrm{Pu}$ and $286-\mathrm{keV} \frac{25}{2}^{+} \rightarrow \frac{21}{2}^{+}$ ${ }^{239} \mathrm{Pu}$ transitions. The evidence seems to indicate that the $227-\mathrm{keV}$ transition belongs to ${ }^{238} \mathrm{Pu}$ (as those transitions appear much stronger in the spectrum than ${ }^{239} \mathrm{Pu}$ transitions do). However, the assignment of the peak at $497-\mathrm{keV}$ to the $22^{+} \rightarrow 20^{+}{ }^{238} \mathrm{Pu}$ transition is questionable, as the transitions between $347-\mathrm{keV}$ and the $497-\mathrm{keV}$ peak are not readily identifiable (ie., the $497-\mathrm{keV}$ peak has more intensity than expected). Nevertheless, a doublet at $497 \mathrm{keV}$ or a background peak leaking through is not excluded.

Figure 3.37 shows the $\gamma$-ray spectrum gated on the $268-\mathrm{keV}$ "mystery" transition, and supplies a bit more ambiguity to the assignment of the "mystery" band to ${ }^{238} \mathrm{Pu}$. Ten clear peaks (and numerous other low statistic candidate peaks) are observed. Table 3.8 shows the assignment of the clear peaks. The strongest peaks belong to ${ }^{238} \mathrm{Pu}$ or the "mystery" band. Other candidate peaks are at $243-\mathrm{keV}\left(\frac{21}{2}^{+} \rightarrow \frac{17}{2}^{+}{ }^{239} \mathrm{Pu}\right), 287-\mathrm{keV}\left(\frac{25}{2}^{+} \rightarrow \frac{21}{2}^{+}{ }^{239} \mathrm{Pu}\right), 327-$ $\operatorname{keV}\left(\frac{29}{2}^{+} \rightarrow \frac{25}{2}^{+}{ }^{239} \mathrm{Pu}\right)$ and $415-\mathrm{keV}\left(\frac{39}{2}^{+} \rightarrow \frac{35}{2}^{+}{ }^{239} \mathrm{Pu}\right)$ mostly belonging to the favored signature of the ground-state band of ${ }^{239} \mathrm{Pu}$. Identification of the "mystery" band as belonging to ${ }^{238} \mathrm{Pu}$ is certainly not unambiguous (due to 
Table 3.8: Peak assignments for the Ge $\gamma$-ray spectrum gated on the 268-keV "mystery" transition observed in the ${ }^{90} \mathrm{Zr}+{ }^{239} \mathrm{Pu}$ experiment

\begin{tabular}{|c|c|}
\hline E (keV) & Source \\
\hline 104 & Pu x-rays \\
145 & "mystery" \\
154 & $\frac{13}{2}^{+} \rightarrow \frac{9}{2}^{+}{ }^{239} \mathrm{Pu}$ \\
185 & "mystery" \\
200 & $\frac{17}{2}^{+} \rightarrow \frac{13}{2}^{+}{ }^{239} \mathrm{Pu}$ \\
227 & "mystery" \\
308 & $12^{+} \rightarrow 10^{+}{ }^{238} \mathrm{Pu}$ \\
347 & $14^{+} \rightarrow 12^{+238} \mathrm{Pu}$ \\
386 & $16^{+} \rightarrow 14^{+238} \mathrm{Pu}$ \\
497 & $22^{+} \rightarrow 20^{+}{ }^{238} \mathrm{Pu}$ \\
\hline
\end{tabular}

the ${ }^{239} \mathrm{Pu}$ transitions seen in some of the "mystery" transition gated spectra); however, as the ${ }^{238} \mathrm{Pu}$ peaks are stronger and consistently observed in Figures $3.34,3.35,3.36$, and 3.37 , these transitions are assigned to ${ }^{238} \mathrm{Pu}$. The possibility of the ${ }^{239} \mathrm{Pu}$ transitions being just background accidentally leaking through the gates is not eliminated.

Therefore, the most consistent picture is that these four "mystery" transitions belong to ${ }^{238} \mathrm{Pu}$ and are part of sume heretofore unobserved side-band in ${ }^{238} \mathrm{Pu}$. The lowest octupole and beta vibrational side.t. nds were extrapolated to higher spins; however, neither extrapolation clearly fits the observed transitions, either in mergy nor spins (based on these transitions having comparable 
spins as ground-state band).

A second possibility, consistent with the $\gamma \cdot \gamma$ coincidence gates, is that the four "mystery" transitions belong to an as yet unobserved super-band or other excited $K=0^{+}$band, and the $496-\mathrm{keV}$ transition connects the superband to the ground-state band. Unfortunately, lack of statistics precludes a more definitive statement on this possibility. 


\section{Theoretical}

\section{Chapter 4}

\section{Introduction}

In order to have a complete understanding of the processes underlying the excitation and population of rotational energy levels, either through Coulomb excitation, inelastic excitation, or neutron transfer reactions, one desires a theory that models the experimental rotational population patterns. Unfortunately, no such simple, elegant theory exists, and the state of nuclear theory in this area of nuclear structure is certainly not good or complete. Most theorists in this area concentrate on semi-classical or classical approaches to the problem, as fully quantum mechanical solutions for such heavy-ion systems require solution of the Schrödinger equation with complicated wavefunctions, and generally require a great many simplifying assumptions. Coulomb excitation, as long as beam energies are safely well below the barrier, is explained fairly well by the semi-classical approach of Alder-Winther-deBoer [73]. This 
method tracks the population amplitudes of rotational levels along a hyperbolic Rutherford trajectory as integration of the time-dependent Schrödinger equations are performed. It seems to handle most dynamical effects well.

However, several complications arise once beam energies are increased, and inelastic excitation or neutron transfer reactions are investigated. Handling of the transfer matrix elements has not been fully derived, and proper inclusion of the nuclear potential has usually been ignored (or assumed to be unimportant).

Many calculations of rotational population patterns in heavy-ion collisions have been performed in the Sudden Approximation limit, where the target nucleus is assumed to remain stationary during the collision. Dynamic effects, and nuclear effects have been ignored, and the fact that the Sudden Approximation tends to overestimate the final maximum spin has been corrected by performing the calculations with a quadrupole moment reduced empirically by $20 \%$ to compensate [74]. In the actinide region, however, this factor was empirically found to be between $10-15 \%$. Such ambiguity of results not only indicates a lack of understanding of tive underlying processes, but is in itself unsatisfactory.

Other methods, such as the Coupled-Channels method [75] and the Classical Limit S-Matrix (CLSM), an alternative semi-classical approach [76],[77],[78], 
do exist for calculating rotational populations but have generally been limited to lighter systems than considered in this thesis. One of the advantages to the CLSM method was thai the nuclear potential could be included easily in the solutions of the equations of motion, and Coulomb-nuclear interference was described more naturally. However, it was practical only for head-on collisions $\left(180^{\circ}\right.$ backscatter $)$, problems with singularities and convergence prevented usage with very heavy systems, and the choice of energy levels and coupling matrix elements was constrained by a classical model. The Coupled-Channels approach also becomes untenable for heavier systems:

This thesis develops some methods for calculating rotational population patterns for systems as heavy as ${ }^{206} \mathrm{~Pb}+{ }^{233} \mathrm{U}$ and ${ }^{90} \mathrm{Zr}+{ }^{239} \mathrm{Pu}$, including nuclear potential effects [79], dynamic effects, and transfer matrix elements, that can be generalized to any scattering angle. Sudden Approximation, MonteCarlo, and semi-classical theories are discussed, with the best calculation being from a modified Alder-Winther-deBoer method. Off-diagonal matrix elements and $\Delta L>0$ transfer (centrifugal effects) are treated. Chapter 5 describes the theories and Chapter 6 compares the results with the experimental data. Also, these methods applied to calculation of the SQUID effects will be briefly discussed [80]. 
Recently, such methods have also been used by Dasso et al. [81] and [82] to calculate Diabolical pair transfer (SQUID effect). They do not include nuclear potential effects nor off-diagonal transfer matrix elements; however, they do include the S-band. Whether population favors the S-band or the ground band after the band crossing is solely dependent on the strength of the mixing matrix elements at the crossing point, something not well calculable by anyone. If the mixing is strong, the Coulomb excited population would tend to remain in the Yrast band, since $B(E 2)$ matrix elements between Yrast states exceed those connecting to the band above Yrast. However, if it is weak, population could go to both bands above the virtual crossing. Mixing matrix elements are difficult to calculate theoretically, and, while we have chosen for simplicity initially not to include the S-band in the calculations, this band is certainly straightforward to include. In addition, the mixing matrix elements could be taken from experiment. 


\section{Chapter 5}

\section{Discussion of Relevant Theories}

This chapter is concerned with discussing several methods used to calculate rotational population patterns for inelastic and neutron transfer reactions. The theories presented in this thesis are all still under development. Figure 5.1 defines the geometry of the physical systems under investigation. The angle $\theta$ is the scattering angle of the projectile, and $\chi$ is the orientation angle of the target axis-of-symmetry with respect to the beam axis. The rotational population pattern calculations using the Sudden Approximation are discussed in Section 5.1; and using modified Alder-Winther-deBoer methods in Section 5.2. 


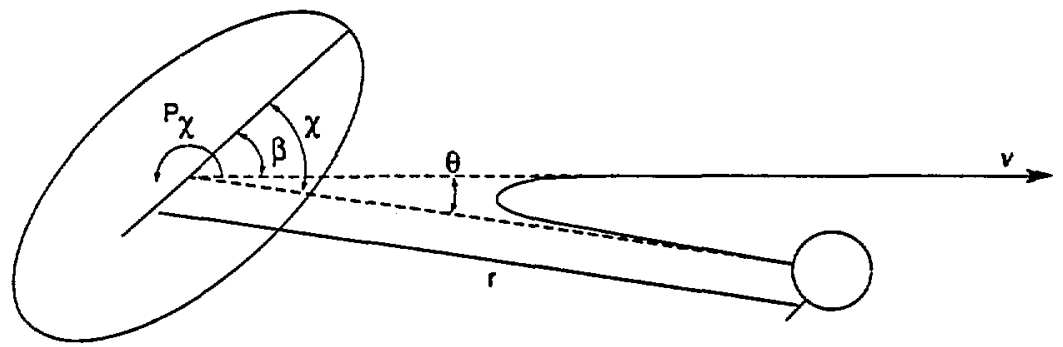

Figure 5.1: Definition of geometry of systems under investigation. 


\subsection{Sudden Approximation}

Perhaps the simplest method that can be devised to calculate the final spin populated in a given reaction is the Sudden Approximation. As the name implies, the target nucleus is assumed not to rotate during the collision, ie., $\chi$ remains constant during the interaction. As will be seen, this approximation is valid for light systems and for obtaining an estimate of the spin at the distance of closest approach. However, other dynamical effects destroy the validity of the method for heavier systems.

The following Monte Carlo method was used to investigate the Sudden Approximation method of calculating final spin populations, and a FORTRAN code called SPIN was developed to utilize this method. Random orientation angles, $\chi_{0}$, were chosen by the computer from a $\sin 2 \chi_{0}$ distribution. In the Sudden Approximation limit, the final spin is given by:

$$
I_{f}=2 q_{2} \sin 2 \chi_{0}
$$

where $x_{0}$ is the initial orientation angle, and $q_{2}$, the quadrupole strength parameter, is given by:

$$
q_{2}=\frac{Z_{p} Q_{0} e^{2}}{4 \hbar v_{0} a^{2}}
$$


where $Q_{0}$ is the quadrupole moment, $e^{2}=1.44 \mathrm{MeV} \cdot \mathrm{fm}, Z_{p}$ is the charge of the projectile, $v_{0}$ is the initial relative velocity at large distance where the interaction is assumed to be aegligible, and $a$ is half the distance of closest approach,

$$
a=\frac{Z_{p} Z_{t} e^{2}}{\mu v_{0}^{2}}
$$

where $Z_{t}$ is the charge ei the target, and $\mu$ is the reduced mass of the system [83]. Hence, the reason for generating random orientation angles from a $\sin 2 \chi$ distribution. It should be noted from equation (5.1) that the maximum final spin possible is associated with a $\chi_{0}=45^{\circ}$, and thus $I_{f \max }=2 q_{2}$.

The change in angular momentum (assume E2's connect rotational energy levels) is given by:

$$
\Delta J=2 \operatorname{NINT}\left(\frac{\Delta J_{\max } \sin 2 \chi_{0}}{2}\right),
$$

where NINT is the operation of taking the nearest integer of the argument in the parenthesis. The possible final spins to be populated are then partitioned according to the Clebsch-Gordan coefficients $\left\langle I \Delta J I 0 \mid I_{\text {int }} I\right\rangle^{2}$, where the Clebsch-Gordan notation used is $\left.\left\langle J_{1} J_{2} m_{1} m_{2}\right| J_{3}-m_{3}\right)^{2} ; I$ is the initial target spin, and $I_{\text {nnt }}$ is the possible half integer spin to be populated. It should be noted that $\sum_{l_{\text {ins }}}\left\langle I J J 0 \mid I_{\text {int }} I\right\rangle^{2}=1$ by definition. The spin then populated 
is:

$$
I_{\text {pop }}=I_{\text {int }}+|\Delta J-I|-1 \text {. }
$$

The results are then histogrammed to generate the Coulomb excitation or inelastic excitation rotational population pattern. For neutron transfer, however, the transferred neutron has some angular momentum, $i$. Transfer of an aligned neutron modifies the change in angular momentum to $\Delta J-i$, as there is a loss of total angular momentum in this case. The anti-aligned neutron results in a change of angular momentum to $\Delta J+i$. The neutron angular momentum, $i$, is calculated using a simple two-level model. The $t$ wo $j_{\frac{15}{2}}$ levels involved in transfer of a neutron in the ${ }^{235} \mathrm{U}$ case are the ${\frac{7^{-}}{2}}^{-}$and the $\frac{5}{2}^{-}$levels (the ground state of ${ }^{235} \mathrm{U}$ is $K^{k}=\frac{7}{2}^{-}$). The cranking Hamiltonian, $H-\frac{\hbar^{2}}{23} \vec{I} \cdot \vec{j}$, can be solved by diagonalizing the $2 \times 2$ matrix:

$$
\left|\begin{array}{cc}
E_{\frac{T}{2}}-w & \frac{\hbar^{2}}{3} \hat{I} \hat{j} \\
\frac{\hbar^{2}}{3} \hat{I} \hat{j} & E_{\frac{5}{2}}-w
\end{array}\right|=0,
$$

where $\dot{I}=\sqrt{I(I+1)-K(K+1)}, \hat{j}=\sqrt{j(j+1)-\Omega(\Omega+1)}, E_{\frac{\frac{T}{2}}{2}}$ is the energy of the $K+1=\Omega+1=\frac{7}{2}^{-}$level, $E_{\frac{5}{2}}$ is the energy of the $K=\Omega=\frac{5}{2}^{-}$ level, $j=\frac{15}{2}$ and $w$ is the eigenvalue to solve for. Note that the off-diagnnal matrix elements are the Coriolis coupling terms. The total angular mome um 
at closest approach is then:

$$
R_{\text {tot }}=R_{\text {core }}+i
$$

The spin populated if an aligned neutron is transferred is:

$$
I_{\text {rans }}=\operatorname{NINT}\left(2 I_{\text {pop }}-i\right),
$$

and that for the transfer of the anti-aligned neutron is:

$$
I_{\text {trans }}=\operatorname{NINT}\left(2 I_{\text {pop }}+i-1\right),
$$

where $i$ is reduced by one unit of angular momentum to attempt to account for the fact that the anti-aligned state is not as strong as the aligned state. Transfer of an aligned ne utron reduces the final angular momentum, and transfer of an anti-aligned neutron increases the final angular momentum of the product. The results are then histogrammed to obtain the neutron transfer population pattern. Typically, 10,000 random orientation angles were chosen. Additionally, to attempt to correct for the overestimation of the final spin in the Sudden Approximation, as the final spin is proportional to the quadrupole moment, $Q_{0}$, calculations were performed with $Q_{0}$ reduced by $15-20 \%$. This reduction of $Q_{0}$ by $20 \%$, however, neglects some very serious dynamical effects, which, as we shall see later, have a large effect on the final population pattern. The 
most serious of these dynamical effects is that the nucleus does rotate during the collision, and thus for some choices of initial orientation angles, can overrotate on the outward path; thus the nucleus experiences a negative torque, and the final spin achieved can be significantly lower than $2 I_{C A}$. Also, the effects of the nuclear potential are ignored. The results from the SPIN code, and a further discussion of the dynamical effects are in Chapter 6 .

The Sudden Approximation was also investigated using the Classical-Limit S-matrix method given by equation 46 of [84]. A FORTRAN code, SMATRIX, was written to atilize the following method. In this method, instead of choosing a random initial orientation angle, $\chi_{0}$, the probability of populating final spin, $I$, can be calculated by numerical integration of:

$$
P_{0 \rightarrow I}=\sqrt{2 J+1} \int_{0}^{1} P_{J}(x) \exp \left(-i \frac{4}{3} q_{2} P_{2}(x)\right) d x
$$

where $P_{J}(x)$ and $P_{2}(x)$ are Legendre polynomials and $x=\cos \chi_{0}$. The probabilities for an even-even nucleus are obtained first. Final probabilities for odd-A nuclei are then given by:

$$
P_{\text {odd-A }}(j)=\sum_{J^{\prime}=I-\frac{1}{2}}^{I_{\max }-\frac{1}{2}}(2 j+1)\left(\begin{array}{ccc}
I_{t} & j^{\prime} & I \\
I_{t} & -I_{t} & 0
\end{array}\right)^{2} P_{e-e}(I),
$$

where $I$ is the even-even spin, $I_{t}$ is the initial target spin and $I_{m a x}$ is the 
maximum even-even spin populated, with the array in parentheses a Wigner $3 j$-symbol. The maximum spin, $I_{\max }$, was usually chosen to be large $(\sim 40 \hbar)$. The probabilitjes for populating even-even levels and odd- $A$ rotational levels can then be obtained for the Coulomb excitation case.

Under the assumption of equal transfer amplitudes over the spheroidal nuclear surface, neutron transfer reactions can be handled by modifying equation $(5.10)$ as in [85]:

$$
P_{T R A N S}(I)=N \sqrt{2 I+1} \int_{0}^{1} P_{l}^{m}(x) a_{t \text { un }}(x) a_{a b s}(x) P_{I}^{0}(x) \exp \left(-i \frac{4}{3} q_{2} P_{2}^{0}(x)\right) d x
$$

where $N$ is a normalization factor given by:

$$
N=(-1)^{m} \frac{\sqrt{2 l+1}(l-m) !}{4 \pi(l+m) !}
$$

and $l$ and $m$ are the quantum numbers of the principal orbit involved in the transfer (for $i_{\frac{13}{2}}$ orbital $l=7, m=3$ ). A tunnelling factor, $a_{t u n}(x)$, is given by:

$$
a_{\text {tun }}(x)=\exp (0.052 r(x))
$$

where $r(x)$ is the transfer distance at an angle $x=\cos \chi_{0}$. The slope factor of 0.052 was obtained by fitting the ${ }^{116} \mathrm{Sn}+{ }^{161} \mathrm{Dy},{ }^{116} \mathrm{Sn}+{ }^{163} \mathrm{Dy}$ and 
${ }^{58} \mathrm{Ni}+{ }^{161} \mathrm{Dy}$ one-neutron transfer $\ln (P)$ vs. $D$ experimental data [86]. Equation (5.12) also includes an absorption factor:

$$
a_{a b s}(x)=\exp \left(\frac{\sqrt{8 \pi \mu a_{w}} A W_{0}}{Z_{p} Z_{t} e^{2} \hbar} \exp \left(\frac{r(x)}{a_{w}}\right)\right),
$$

where the diffuseness, $a_{w}=0.54 \mathrm{fm}$ and the imaginary part of the nuclear potential, $W_{0}=25 \mathrm{MeV}$. Probabilities for one-neutron transfer from odd-A to even-even nuclei can then be calculated by numerical integration of equation (5.12). Additional factors can be included in equation (5.12) to calculate two-neutron transfer of the SQUID effect-in fact, Canto et al. include a spectroscopic form factor, $a_{\text {spec }}$, in reference [85], to take into account microscopic nucleon structure in the nuclear SQUID effect. A discussion of a new SQUID calculation, and results from SMATRIX can be found in Chapter 6 .

\subsection{Alder-Winther-deBoer modified method}

\subsubsection{General Description}

The general formalism of the Alder-Winther-deBoer method can be found in [73], and there exists a FORTRAN code for calculating Coulomb excitation 
probabilities [87]. The projectile is assumed to move on a classical Rutherford trajectory, $r(t)$, and the structure of the projectile and target in different channels is given by the Hamiltonian:

$$
H_{\circ}|n\rangle=\epsilon_{n}|n\rangle,
$$

where $\epsilon_{n}$ are the eigenvalues. $H_{0}$ includes no interaction between the projectile and target and thus $|n\rangle$ is a product of projectile and target wavefunctions:

$$
|n\rangle=\left|\Psi_{1}\right\rangle\left|\Psi_{p}\right\rangle
$$

At any time, $t$, the wavefunction can be expanded in terms of these eigenstates:

$$
|\Psi(t)\rangle=\sum_{n} a_{n}(t) e^{i e_{n} t} \mid n j,
$$

where $a_{n}(t)$ are time-dependent amplitude coefficients.

When the projectile and target collide, this produces an interaction potential $V(t)$ which causes excitation along the band of eigenstates. The timedependent Hamiltonian is:

$$
H(t)=I_{\mathrm{o}}+V(t)
$$

and the coupled time-dependent Schrödinger (TDS) equations can be written:

$$
i \frac{d}{d t} a_{,}(t)=\sum_{1}\langle j|V(t)| i\rangle e^{i\left(\ell_{,}-\epsilon_{0}\right) t} a_{i}(t),
$$


where $a_{j}(t)$ and $a_{i}(t)$ are the rotational state population amplitudes. Planck's constant $\hbar$ is considered unity in this expression. The Alder-Winther-deBoer method solves the TDS equations with the initial condition

$$
a_{n}(t=-\infty)=\delta_{n 0}
$$

with only the lowest rotational amplitude being non-zero before the collision. The final probabilities at $t=\infty$ are:

$$
P_{f}(\infty)=\left|a_{f}(\infty)\right|^{2} .
$$

In all cases here excitation of the projectile is neglected. This is a reasonable first approximation, as in most cases experimental projectiles have been chosen to be closed-shell nuclei (or closed-subshell), and thus they have fairly high first excited states. The interaction potential, $V(t)$, can be expanded in terms of spherical harmonics

$$
V(r(t))=\sum_{\mu \nu} V_{\mu \nu}(r) Y_{\mu \nu}^{*}(\hat{r}),
$$

and equations (5.20) can be rewritten as:

$$
i \frac{d}{d t} a,(t)=\sum_{i} \sum_{\mu \nu}(-1)^{J,-M,}\left(\begin{array}{ccc}
I_{j} & \lambda & I_{i} \\
-M_{j} & \mu & M_{i}
\end{array}\right)\left\langle j\left\|V_{\lambda}(r(t))\right\| i\right\rangle e^{i\left(\epsilon_{2}-\epsilon_{1}\right) t} a_{i}(t),
$$

Where $\left\langle j\left\|V_{\lambda}(r(t))\right\| i\right\rangle$ is the reduced matrix element. 


\subsubsection{Coulomb Excitation}

For Coulomb excitation, the matrix element $\langle j\|V(t)\| i\rangle$ is determined as follows. First, the interaction is calculated in the budy-fixed (rotating) frame of the target nucleus and expanded in terms of spherical harmonics:

$$
V\left(r^{\prime}\right)=\sum_{\lambda \nu^{\prime}} V_{\lambda \nu^{\prime}}(r) Y_{\lambda \nu^{\prime}}^{*}\left(\hat{r}^{\prime}\right),
$$

where the primes denote the rotating frame. Because the solution of the equation of motion is performed in the lab frame, the potential is transformed to the lab system by rotation $R(\Omega)$ in the Euler angles $\Omega$ :

$$
V(r)=\sum_{\lambda \mu \mu^{\prime}} V_{\lambda \mu^{\prime}}(r) Y_{\lambda \mu}^{\ddot{x}^{\prime}}(\hat{r}) D_{\mu \mu^{\prime}}^{\lambda}(\Omega) .
$$

Integration over all Euler angles yields a matrix element:

$$
\begin{aligned}
& \langle f|V(r)| i\rangle=\left\langle A_{f} I_{f} M I_{f}|V(r)| A_{i} I_{i} M_{i}\right\rangle= \\
& \sum_{\lambda \mu}(-1)^{l_{f}-M_{f}}\left(\begin{array}{ccc}
I_{f} & \lambda & I_{i} \\
-M_{f} & \mu & M_{i}
\end{array}\right) Y_{\lambda \mu}^{*}(\hat{r})\left\langle A_{f} I_{f}\left\|V_{\lambda}(r)\right\| A_{i} I_{i}\right\rangle,
\end{aligned}
$$

where the reduced matrix element is defined as:

$$
\begin{aligned}
& \left\langle A_{f} I_{f}\left\|V_{\lambda}(r)\right\| A_{i} I_{i}\right\rangle=\sum_{\kappa_{j} K_{0} \mu^{\prime}} \sqrt{2 I_{f}+1} \sqrt{2 I_{i}+1} \\
& (-1)^{I_{f}-K_{j}}\left(\begin{array}{ccc}
I_{f} & \lambda & I_{i} \\
-K_{j} & \mu & K_{i}
\end{array}\right)\left\langle\Phi_{K_{j}}^{f}\left|V_{\lambda \mu^{\prime}}(r)\right| \Phi_{K_{i}}^{i}\right\rangle,
\end{aligned}
$$


where $\Phi_{K}^{f}$ and $\Phi_{K_{1}}^{i}$ are the intrinsic eigenstates of the z-component of angular momentum of the target. For the simple case of a head-on collision, $\theta=\pi$, the spherical harmonic $Y_{\lambda \mu}(\hat{r})=\delta_{\mu 0} \sqrt{(2 \lambda+1) / 4 \pi}$ and $M_{J}=M_{i}$. Results are shown in Chapter 6.

\subsubsection{Inelastic excitation}

For inelastic excitation, one must include the nuclear part of the potential, $V^{N}(t)$ as well as the Coulomb potential, $V^{C}(t):$

$$
V(t)=V^{C}(t)+V^{N}(t)
$$

The Coulomb potential can be written in a multipole expansion:

$$
V_{\lambda \mu^{\prime}}^{C}(r)=\frac{Z_{p} e^{2}}{r^{\lambda+1}} \frac{4 \pi}{2 \lambda+1} Q_{\lambda \mu^{\prime}}^{i n t}
$$

where $Q_{\lambda \mu^{\prime}}^{\text {int }}$ are the intrinsic multipole moments.

The nuclear potential is taken from the standard deformed optical model potentials:

$$
V^{N}\left(r^{\prime}\right)=\frac{-(U+i W)}{1+\exp \left[\left(r-R_{p}-R_{t}\left(\dot{r}^{\prime}\right)\right) / a\right]},
$$

where $U$ and $W$ are the real and imaginary potential well depths, respectively, 
and $R_{t}$ can be expressed, for an axially symmetric nucleus, as:

$$
R_{t}\left(\chi^{\prime}\right)=R_{t}\left(1+\sqrt{\frac{5}{4 \pi}} \beta P_{2}\left(\cos \chi^{\prime}\right)\right)
$$

where $R_{t}=r_{0} A_{t}^{\frac{2}{3}}$ and $\beta$ is the deformation parameter. The nuclear potential can also be written in terms of spherical harmonics and transformed to the lab frame:

$$
V_{\lambda 0}^{N}=\sqrt{4 \pi(2 \lambda+1)} \frac{1}{2} \int_{-1}^{1} \frac{-(U+i W)}{1+\exp \left[\left(r-R_{p}-R_{t}(\chi)\right) / a\right]} P_{\lambda}(\cos \chi) d(\cos \chi) .
$$

The reduced matrix elements, for fixed $K$, are:

$$
\begin{aligned}
& \left\langle A_{f} I_{f}\left\|V_{\lambda}^{C}(r)+V_{\lambda}^{N}(r)\right\| A_{i} I_{i}\right\rangle= \\
& \quad \sqrt{2 I_{f}+1} \sqrt{2 I_{i}+1}(-1)^{I_{f}-k}\left(\begin{array}{ccc}
I_{f} & \lambda & I_{i} \\
-K^{\prime} & \mu & I^{*}
\end{array}\right) V_{\lambda 0}(r) .
\end{aligned}
$$

However, inclusion of $V^{N}(r)$ causes a distortion of the Rutherford trajectory. This cannot be handled exactly in the Alder-Winther-deBoer formulation. However, since the quadrupole torque from electric and nuclear forces is largest at closest approach, it is important that the Rutherford trajectory be nearly correct at closest approach. We do this by choosing an effective beam energy and target $Z$. Thus, a method of reproducing the turning point and 
proper force at this point by reducing the lab beam energy and effective charge of the spheroidal collision partner is developed. The intrinsic quadrupole moment is fixed so that torque from electromagnetic forces remains unaltered. The turning point, $r_{C A}$, is found by numerically solving the equation:

$$
E_{C M}=\frac{Z_{p} Z_{t} e^{2}}{r_{C A}}+U\left(r_{C A}\right)
$$

where $E_{C M}$ is the beam energy in center-of-mass reference frame, and $U\left(r_{C A}\right)$ is the real part of the nuclear optical potential, $V^{N}(r)$. The radial force, $F_{C A}$, is simply the negative derivative of the full potential:

$$
F_{C A}=\frac{Z_{p} Z_{t} \epsilon^{2}}{r_{C A}^{2}}-\left(\frac{d U}{d r}\right)_{r C A}
$$

The effective charge, $Z_{e f f}$, and the effective energy, $E_{e f f}$, are then given by:

$$
\frac{Z_{p} Z_{e \int \rho} e^{2}}{r_{C A}^{2}}=F_{C A}
$$

and

$$
E_{e f f}=\frac{Z_{\mathrm{p}} Z_{e f f} e^{2}}{r_{C A}}
$$

The Alder-Winther-deBoer method run with these reduced $E_{\text {efs }}$ and $Z_{e f s}$ parameters more closely matches [i9] the full coupled channel results for ${ }^{40} \mathrm{Ar}+{ }^{160} \mathrm{Gd}$ of reference [75]. Results are shown in Chapter 6. 


\subsubsection{Neutron Transfer}

Two methods of applying modified Alder-Winther-deBoer calculations shall be described. The first method involves running the Alder-Winther-deBoer method described in the Coulomb Excitation Section (5.2.2) and multiplying the occupation amplitudes at closest approach by a transfer matrix, $T_{f^{\prime} f}$. It is generally accepted that the neutron transfer process is localized in time and space around the point of closest approach, $t=0$, in the calculation. $T_{d^{\prime} I}$ is obtained by integration of:

$$
T_{I^{\prime} I}=T_{0} \int_{-1}^{1} P_{I^{\prime}}(x) a_{\mathrm{tun}}(x) a_{a b s}(x) F_{I^{\prime} I}(x) P_{I}(x) d x,
$$

where $P_{I^{\prime}}(x)$ and $P_{r}(x)$ are Legendre polynomials, $a_{t u n}(x)$ and $a_{a b s}(x)$ are the tunnelling and absorption terms described in the Sudden Approximation Section $5.1, T_{0}$ is the normalization factor between transfer from target to projectile and the time reversed reaction $\left(T_{0}=1\right.$ for our cases since the ratio is unknown), and $F_{I^{\prime}}(x)$ is the angular transfer form factor at the inner turning point, $r_{C A}$. For example, the two-neutron angular form factor is given by:

$$
F_{I^{\prime} I}(x)=\left\langle A+2, I^{\prime}\left|S^{\dagger}\left\langle r_{C A}\right)\right| A, I\right\rangle .
$$

The intrinsic states $\left|A+2, I^{\prime}\right\rangle$ and $|A, I\rangle$ are approximated by Cranked HartreeFock-Bogoliubov (CHFB) functions and $F_{I r}(x)$ is calculated with the $S^{\dagger}\left(r_{C A}\right)$ 
operator being a pair creation/annihilation operator (or single neutron creation/annihilation operator for one-neutron transfer). The angular form factors represent the structure of the target, specifically, the site dependence of transfer on the deformed target surface. If one neglects pairing and the dependence of the intrinsic wavefunction on the collective angular momentum, and assumes the neutron(s) are transferred to a particular Nilsson orbital (governed by the target wavefunction), $F_{I^{\prime} I}(x)$ is proportional to the $\left|\sum Y_{l m}(\theta \phi)\right|^{2}$, where $\theta$ and $\phi$ are the polar angles. For example, pair transfer to the $i_{\frac{13}{3}}$ intruder shell in the rare-earths yields a form factor, $F_{l^{\prime} I}(x)=\left|Y_{6 m}(\theta \phi)\right|^{2}$, as shown in reference [88]. In this thesis, both the approximation of spherical harmonics and CHFB functions are used.

Thus, the method is to run the integration of amplitudes from $t=-\infty$ to $t=0$, stop the calculation, multiply by $T_{I^{\prime} t}$ as follows:

$$
a_{l}^{\prime}(0)=\sum_{I} T_{I^{\prime} l} a_{l}(0)
$$

and after the multiplication, continue the time evolution with the $a_{I}^{\prime}(0)$ amplitudes. The final probabilities are given as in equation (5.22).

Because $T_{I^{\prime} t}$ cont ains significant off-diagonal matrix elements, representing I ransfer of ncutron(s) with angular momentum $\left(\Delta I=I^{\prime}-I\right)$, there are several 
additional factors to be considered. First, there is a centrifugal barrier which will add to the tunnell:.ıg factor $a_{\text {tun }}(x)$. Second, there is an effect due to the finite size of the projectile. Indeed, since a heavy-ion projectile is not a point-like probe of a varying $F_{l^{\prime} I}$ function, there will be some angular smeating over the surface. These effects are estimated by considering a square tunnelling barrier between th e nuclear surfaces at the classical turning point. Two factors are obtained:

$$
g_{1}(\Delta I)=\exp \left(-\left(\left[4 m_{n}\left(B_{C E N T}+S\right)\right]^{\frac{1}{2}}-\left(4 m_{n} S\right)^{\frac{1}{2}}\right)\left(\frac{r_{C A}-R_{t}-R_{p}}{\hbar}\right)\right),
$$

where $m_{n}$ is the ncutron mass, $S$ is the one- or two-neutron separation energy, $r_{C A}$ is the distanc of closest approach, and

$$
B_{C E N T}=\frac{\hbar^{2} \Delta I(\Delta I+1)}{4 m_{n} R_{i}^{2}}
$$

and

$$
g_{2}(\Delta I)=\exp \left[-\left(\frac{y \Delta I}{R_{t}}\right)^{2}\right],
$$

where $y$, the characteristic width parameter of the tunnelling wave packet is:

$$
y=(2 \hbar)^{\frac{1}{2}}\left(4 m_{n} S\right)^{-\frac{1}{4}}\left(\frac{1}{R_{t}}+\frac{1}{R_{p}}\right)^{-\frac{1}{2}} .
$$

For inclusion of these factors, the $T_{I^{\prime \prime}}$ in equation (5.39) are multiplied by $g_{1}$ and $g_{2}$. Results from these calculations are presented in Chapter 6 . 
The second method involves a more direct evaluation of the $\left\langle f\left|V^{t r}(r)\right| i\right\rangle$ matrix element; and does not limit the transfer to $t=0$. For pair transfer,

$$
\left(f\left|V^{t r}(r)\right| i\right\rangle=T_{0}\left\langle f\left|B^{\dagger}(r)\right| i\right\rangle
$$

where $B^{\dagger}(r)$ is a boson (Cooper pair of neutrons) creation operator. As usual, $B^{\dagger}(r)$ can be decomposed in terms of spherical harmonics, and thus the reduced matrix element can be written:

$$
\begin{gathered}
\left\langle A_{f} I_{f}\left\|V_{\lambda}^{t r}(r)\right\| A_{i} I_{i}\right\rangle=T_{0} \sum_{K_{j} K_{\mu^{\prime}} \mu^{\prime}} \sqrt{2 I_{j}+1} \sqrt{2 I_{i}+1} \\
(-1)^{I_{j}-K_{j}}\left(\begin{array}{ccc}
I_{j} & \lambda & I_{i} \\
-K_{j} & \mu^{\prime} & K_{i}
\end{array}\right)\left\langle\Phi_{K^{\prime}}^{\prime}\left|B_{\lambda \mu^{\prime}}^{\dagger}(r)\right| \Phi_{K_{i}}^{i}\right\rangle
\end{gathered}
$$

where

$$
B_{\lambda \mu^{\prime}}^{\dagger}(r)=\int B^{\dagger}(\hat{r}) Y_{\lambda \mu}(\hat{r}) d^{2} \hat{r}
$$

These matrix elements contain information on the structure of nuclear states in the rotaiional band. Because these matrix elements are needed at very large radii, $r$, where the oscillator functions used for the calculation of the intrinsic many-body wavefunctions are not reliable, and decomposition of the intrinsic wavefuntion with respect to quantum number $K$ requires a projection, two approximations are used. To address the problem with the wavefunctions, a 
penetrability factor is introduced. Since it is difficult to express the actual wavefunction in terms of microscopic wavefunctions at large distance, $r$, it is approximated in this region by a wavefunction $\phi(r, E)$ of a single boson in a deformed Woods-Saxon well $U(r)$ with mass, $m_{B}$, and energy $E$, determined by the binding energy of the boson in the projectile. This wavefunction can then be decompcsed in spherical harmonics:

$$
\phi(\hat{r}, E)=\frac{1}{r} \sum_{l m} \phi_{l m}(r, E) Y_{l m}(\hat{r})
$$

and the coupled-channels equation

$$
\begin{aligned}
& {\left[-\frac{1}{2 m_{b}} \frac{d^{2}}{d r^{2}}+\frac{l(l+1)}{r^{2}}+U_{0}(r)-E\right] \phi_{l m}(r, E)=} \\
& -\sum_{\lambda>0_{\mu}} \sum_{l^{\prime} \mu^{\prime}} U_{\lambda \mu}(r) \frac{\sqrt{2 l+1} \sqrt{2 \lambda+1} \sqrt{2 l^{\prime}+1}}{\sqrt{4 \pi}}(-1)^{\pi} \\
& \left(\begin{array}{lll}
l & \lambda^{\prime} & l^{\prime} \\
0 & 0 & 0
\end{array}\right)\left(\begin{array}{ccc}
l & \lambda & l^{\prime} \\
-m & \mu & m^{\prime}
\end{array}\right) \phi_{l^{\prime} m^{\prime}}(r, E)
\end{aligned}
$$

for these wavefunctions. A matching radius, $R$, is chosen to be large enough to be outside the range of the potential, $U$, but small enough that the representation of the wavefunction in terms of $\phi$ is still reliable. Thus,

$$
o_{l m}(r, E)=P_{l}(r, R, E) \phi_{l m}(R)
$$


where the penetrability, $P_{1}(r, R, E)$ in the WKB approximation is:

$$
P_{i}(r, R, E)=\exp \left[-\int_{R}^{r} \sqrt{\frac{l(l+1)}{r^{\prime 2}}-2 m E} d r^{\prime}\right] .
$$

The reduced transition matrix element is then:

$$
\left\langle f\left\|B_{\lambda}^{\dagger}(r)\right\| i\right\rangle=P_{\lambda}\left(r-R_{p}, R, E\right)\left\langle f\left\|B_{\lambda}^{\dagger}(R)\right\| i\right\rangle,
$$

where the $r$ is replaced by $r-R_{p}$ because the transfer takes place mostly at the surface of the projectile.

The second approximation, the high-spin approximation, for large $I$ and small $K(K \ll I$ and $\lambda \ll I)$, makes use of the semiclassical expression for the Wigner coefficient

$$
\sqrt{2 I_{J}+1}(-1)^{I_{j}-K_{J}}\left(\begin{array}{ccc}
I_{J} & \lambda & I_{i} \\
-K_{J} & \mu & K_{i}
\end{array}\right)=d_{\mu \Delta I}^{\lambda}\left(-\frac{\pi}{2}\right),
$$

where $\Delta I=I_{J}-I_{i}$ is the angular momentum transfer and $d_{\mu \Delta I}^{\lambda}\left(-\frac{\pi}{2}\right)$ describes a rotation of $90^{\circ}$ around the $y$-axis. Thus, the reduced transition matrix elements can be written:

$$
\left\langle A_{f} I_{f}\left\|V_{\Lambda}^{\mathrm{tr}}(R)\right\| A_{i} I_{i}\right\rangle=T_{0} \sqrt{2 I_{i}+1}\left\langle\Phi^{J}\left|\hat{B}_{, \Delta \Delta}^{\dagger}(R)\right| \Phi^{i}\right\rangle,
$$

where

$$
\tilde{B}_{\lambda_{\mu}}^{\dagger}=\sum_{\mu^{\prime}} d_{\mu^{\prime} \mu}^{\lambda}\left(-\frac{\pi}{2}\right) B_{\lambda_{\mu} \mu^{\prime}}^{\dagger}
$$


The matrix elements $\left\langle\Phi^{f}\left|B_{\lambda \mu}^{\dagger}(R)\right| \Phi^{i}\right\rangle$, again can be found through decomposition of the angular form factor given below in terms of spherical harmonics:

$$
F(R) \equiv\left\langle\Phi^{f}\left|B_{\lambda_{\mu}}^{\dagger}(R)\right| \Phi^{i}\right\rangle=\sum_{\lambda \mu} a_{\lambda \mu}(R) Y_{\lambda_{\mu}}^{*}(\hat{R})
$$

where $\hat{R}$ is a vector with the length of the matching radius, $R$. Thus,

$$
a_{\lambda \mu}(R)=\left\langle\Phi^{J}\left|B_{\lambda \mu}^{\dagger}(R)\right| \Phi^{i j}\right\rangle
$$

and has the property

$$
a_{\lambda \mu}(R)=(-1)^{\lambda} a_{\lambda(-\mu)}(R) .
$$

Similarly, an $\tilde{a}_{\lambda_{\mu}}(R)$ quantized along the $\mathrm{x}$-axis can be defined:

$$
\tilde{a}_{\lambda \mu}(R)=\left\langle\Phi^{J}\left|\tilde{B}_{\lambda \mu}^{\dagger}(R)\right| \Phi^{i}\right\rangle
$$

where

$$
\tilde{a}_{\lambda \mu}(R)=(-1)^{\lambda} \tilde{a}_{\lambda(-\mu)}(R)
$$

and $\bar{a}_{\lambda_{\mu}}(R)=0$ for odd values of $\mu$. The coefficients, $a_{\lambda \mu}(R)$, can be found with some Clebsch-Gordan algebra in terms of a transition density matrix, 
$F_{n n^{\prime}}:$

$$
\begin{gathered}
a_{\lambda \mu}(R)=\sum_{n n^{\prime \prime \prime} m^{\prime} m^{\prime}} F_{n l j m, n^{\prime \prime} j^{\prime} m^{\prime}} R_{n l}(R) R_{n^{\prime \prime} l^{\prime}}(R)(-1)^{l+j-\frac{1}{2}+\mu} \\
. \frac{\sqrt{2 l+1} \sqrt{2 l^{\prime}+1} \sqrt{2 j+1} \sqrt{2 j^{\prime}+1} \sqrt{2 \lambda+1}}{\sqrt{8 \pi}} \\
\cdot\left(\begin{array}{lll}
l & \lambda & l^{\prime} \\
0 & 0 & 0
\end{array}\right)\left(\begin{array}{ccc}
j & \lambda & j^{\prime} \\
m & -\mu & m^{\prime}
\end{array}\right)\left\{\begin{array}{ccc}
j & \lambda & j^{\prime} \\
l^{\prime} & \frac{1}{2} & l
\end{array}\right\}
\end{gathered}
$$

where $R_{n l}(R)$ and $R_{n \prime \prime \prime}(R)$ are radial wavefunctions and the array in curly brackets is the Wigner $6 j$ symbol. Finaliy, the reduced transition matrix element can be written as:

$$
\left\langle A_{f} I_{f}\left\|V_{\lambda}^{\mathrm{tr}}(r)\right\| A_{i} I_{i}\right\rangle=T_{0} \sqrt{2 I_{i}+1} P_{\lambda}\left(r-R_{p}, R, E\right) \tilde{a}_{\lambda\left(I_{f}-l_{1}\right)}(R)
$$

Note, the $P_{\lambda}\left(r-R_{p}, R, E\right)$ term includes centrifugal effects more naturally in this formalism.

For one-neutron transfer, from an odd-A nucleus to an even-even nucleus, a similar derivation can be used. Basically, one merely has to include the odd-A odd-particle with spin:

$$
\left|\psi_{i}^{(t)}\right\rangle=\left|m_{s}\right\rangle\left|\psi_{f}^{(t)}\right\rangle
$$


Thus, the matrix elements are:

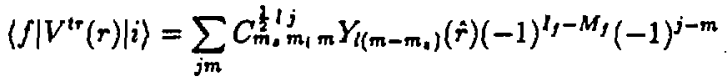

$$
\begin{aligned}
& \left(\begin{array}{ccc}
I_{J} & j & I_{i} \\
-M_{J} & -m & M_{i}
\end{array}\right)\left\langle f\left\|V_{j}^{t r}(r)\right\| i\right\rangle,
\end{aligned}
$$

and the reduced matrix element is:

$$
\begin{aligned}
& \left\langle f\left\|V_{j}^{t \tau}(r)\right\| i\right\rangle=\sqrt{2} \sqrt{2 I_{i}+1} \sqrt{2 I_{j}+1} \\
& \sum_{n} C_{n l_{j}}^{K_{l}} R_{n l}(r)(-1)^{I_{f}+j-K_{i}}\left(\begin{array}{ccc}
I_{j} & j & I_{i} \\
0 & -K_{i} & K_{i}
\end{array}\right),
\end{aligned}
$$

where $C$ 's are Casimir coefficients and

$$
R_{\mathrm{n} l}(r)=P_{l}\left(r-R_{\mathrm{p}}, R, E\right) R_{\mathrm{n} l}(R)
$$

according to the approximations discussed above. 


\section{Chapter 6}

\section{Comparison of Theory with Experimental Data}

This chapter will compare the results of the theoretical methods discussed in Chapter 5 with the experimental data. Historically, it has been difficult to compare experimental data with theoretical calculations because experiments were run at specific beam energies, scattering angles, etc. and calculations have mostly been for head-on collisions and have neglected nuclear potential effects, scattering angle effects, etc. This is reflected in the large number of intra-theory comparisons and small number of theory-experiment comparisons. As a first comparison of data to theory, to just introduce the effectiveness of the theoretical methods in Chapter 5, a brief discussion of the SQUID effect and the results of the calculations will be presented and compared with a ${ }^{156} \mathrm{Gd}+{ }^{206} \mathrm{~Pb}$ experiment that is the work of Helmer et al. [89] (see Section 
6.1). Then, the rotational population patterns will be presented for the ${ }^{58} \mathrm{Ni}$ $+{ }^{233} \mathrm{U}$ experiment (Section 6.2), the ${ }^{208} \mathrm{~Pb}+{ }^{233} \mathrm{U}$ experiment (Section 6.2), and the ${ }^{90} \mathrm{Zr}+{ }^{239} \mathrm{Pu}$ experiment (Section 6.3 ), and compared to theoretical calculations.

\subsection{Brief SQUID Results}

The Superconducting Quantum Interference Device (SQUID) effect is discussed more completely in the literature [90],[91]. Briefly, it can be described as follows. Because the Coulomb force is a long range force, and nucleon transfer occurs with the highest probability at the distance of closest approach in a given heavy-ion nuclear reaction, the nucleon transfer tends to occur from rotationally excited states. If Coulomb excitation can pump in enough angular momentum to excite the target nucleus to the backbending region (or diabolical point) on the inward path of the projectile, two transfer paths exist: (1) The inward-path Coulomb excitation adds angular momentum to just below the diabolical point, nucleon transfer occurs below that point, and then the product nucleus is again Coulomb excited on the outward path of the projectile; or (2) The inward-path Coulomb excitation adds angular momentum to 
just above the diabolical point, nucleon transfer occurs above that point, and then the product nucleus is Coulomb excited on the outward path. These two transfer paths quantum interfere. Nikam and Ring [92] found that the pair transfer matrix elements not only tended to sharply decrease in the region around the diabolical point, but also went negative. The experimental signature of this effect would be a sharp decrease in the population probability, and there have been several attempts to establish the region in which such an effect would manifest itself $([15],[80],[85])$. Originally, because many calculations were performed in the Sudden Approximation limit, it was thought that the sharp decrease would occur at a spin twice that of the diabolical or backbending point, and little hope was held for experimental observation of the SQUID effect. The backbending point in the rare earths is $\sim 12-14 \hbar$, thus one would need to observe at least to spin $24-28 \hbar$, and even higher to be able to distinguish (1) a large decrease in the population probability from (2) a lack of statistics. The situation is even worse in the actinides, as the backbending point tends to be even higher in spin $(\sim 30 \hbar)$. Our most recent calculations [93], however, hold out the hope that perhaps a SQUID effect could be observed at lower spins (as low as $10-14 \hbar$ ).

It is not the purpose of this thesis to completely discuss the SQUID effect 
and the calculations, rather, it is the intention to present the results as an illustration of the theoretical model of Chapter 5 Section 5.2.4 and to compare with a recent experiment as a springboard for comparisons in the actinide region. Figures 6.1 and 6.2 show the calculated results using the method of Section 5.2.4 for the ${ }^{208} \mathrm{~Pb}+{ }^{160} \mathrm{Dy}$ (Figure 6.1) and ${ }^{208} \mathrm{~Pb}+{ }^{158} \mathrm{Dy}$ (Figure 6.2) neutron pair transfer reactions. In both, the beam energy was $1100 \mathrm{MeV}$, the population probabilities at spin 0 are normalized to unity, and the scattering angle is $180^{\circ}$. Figure 6.1 corresponds to a case with a diabolical point, anc Figure 6.2 corresponds to a case without a diabolical point. The dotted tine (square symbols) is the calculation performed for the case where the $t_{i}$. .... $F_{I^{\prime} I}(x)$ (see Section 5.2.4) are set to unisy, and the full line $(\bullet)$ corresponds to a calculation performed with the full transfer matrix $T_{I^{\prime} I}$. A comparison of Figures 6.1 and 6.2 shows the large dip in population probability starting around spin 10 , but enlarging at spins $14-16$, for the case in which a diabolical point exists. From numerous other calculations with different combinations of input parameters not shown here, this dip at spin $I \approx 12 \hbar$ is fairly robust.

Figure 6.3 compares the solid line $(\bullet)$ of Figures 6.1 and 6.2 with experimental two-neutron transfer yields in the sance region in the rare earths from the ${ }^{156} \mathrm{Gd}+{ }^{206} \mathrm{~Pb}$ reaction. Pair transfer yields as a function of spin are pre- 


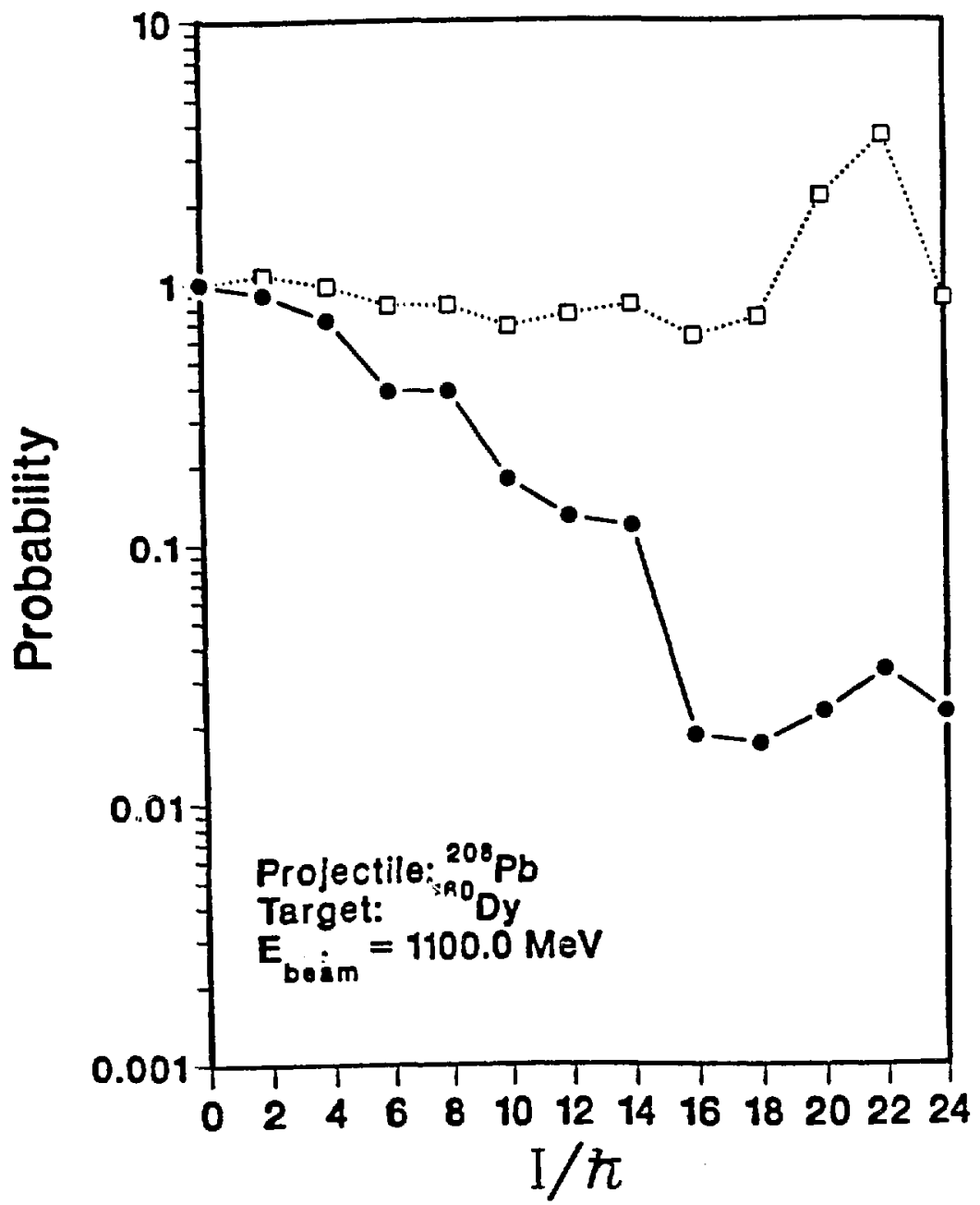

Figure 6.1: Theoretical calculation of the rotational population probability for states in the grourd band in the neutron pair transfer reaction ${ }^{208} \mathrm{~Pb}+{ }^{160} \mathrm{Dy}$ reaction. The dotted line (open squares) is the calculation performed for the case where the functions $F_{l^{\prime}}(x)$ (see Section 5.2.4) are set to unity, and the full line ( $\bullet$ corresponds to a calculation performed with the full transfer matrix $T_{\left.l^{\prime}\right]}$. 


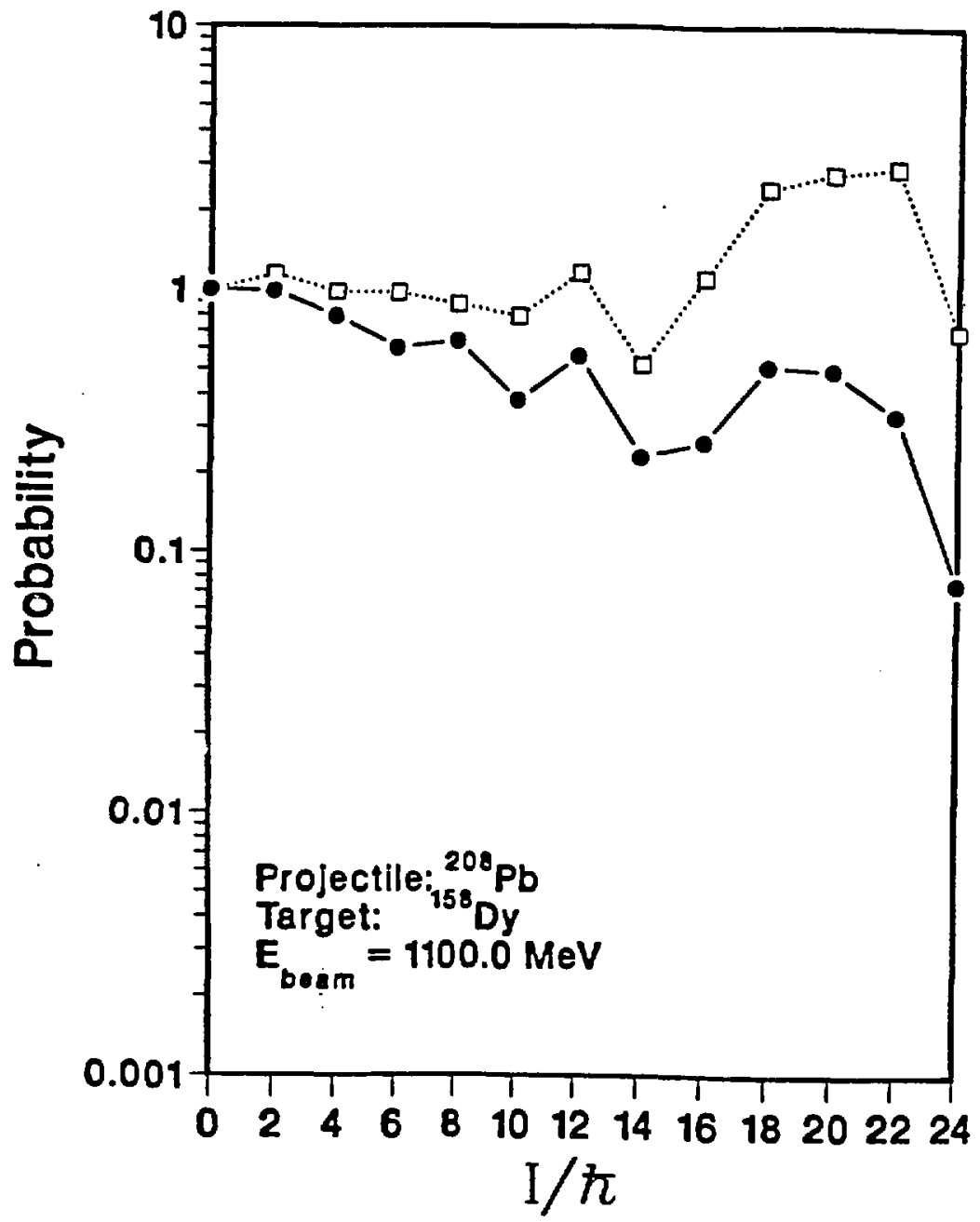

Figure 6.2: Theoretical calculation of the rotational population probability for states in the ground band in the neutron pair transfer reaction ${ }^{208} \mathrm{~Pb}+{ }^{158} \mathrm{Dy}$ reaction. The symbols are the same as for Figure 6.1. 


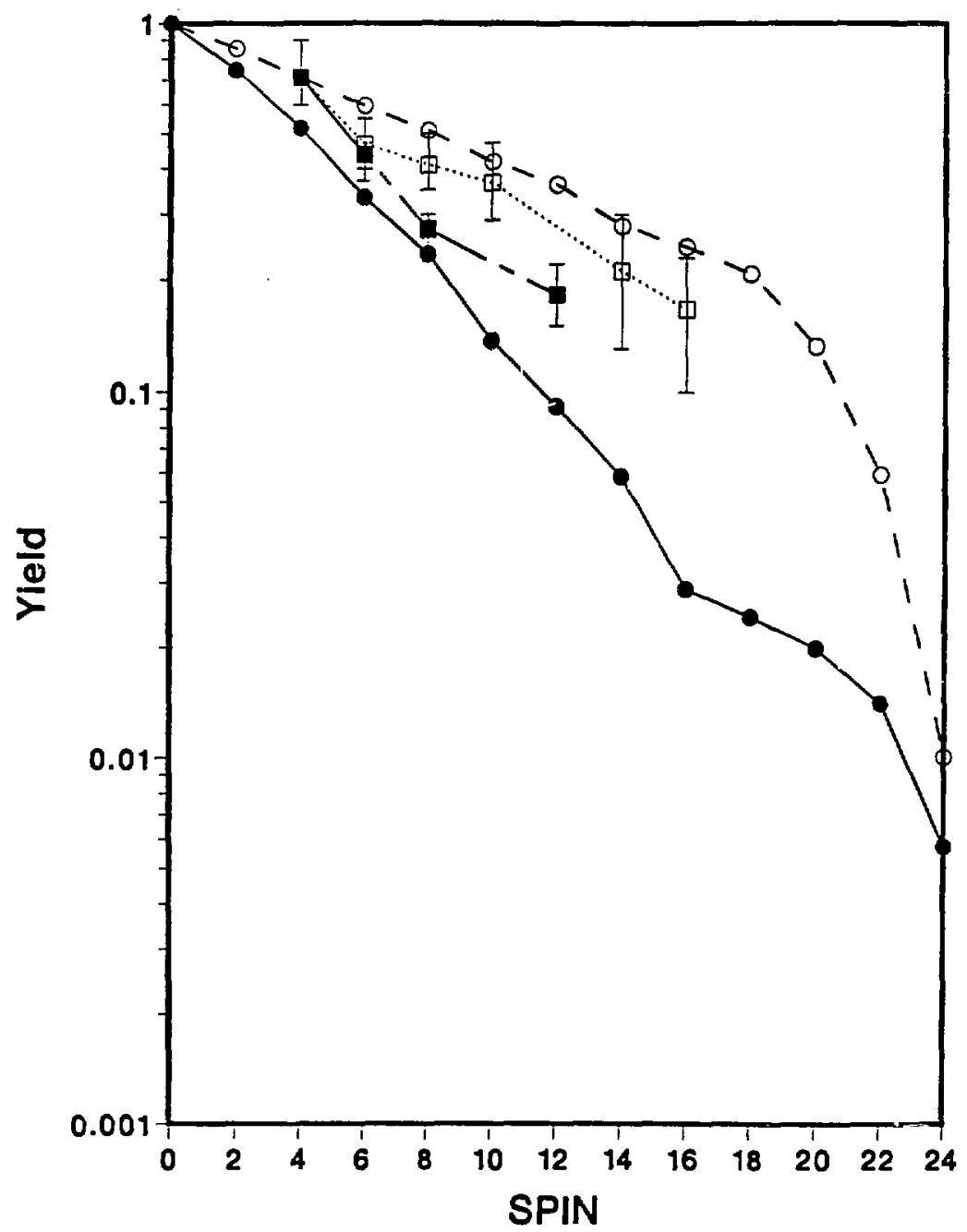

Figure 6.3: A comparison of the experimental rotational population yields extracted from the reaction ${ }^{156} \mathrm{Gd}+{ }^{206} \mathrm{~Pb}$ with the calculations of theory from Section 5.2 .4 for the reactions ${ }^{208} \mathrm{~Pb}+{ }^{160} \mathrm{Dy}$ and ${ }^{208} \mathrm{~Pb}+{ }^{158} \mathrm{Dy}$. The solid line $(\bullet)$ presents the case with the diabolical point, the dashed line (open circles) presents the case without a diabolical point, the dotted line (open squares) is data for ${ }^{154} \mathrm{Gd}$ and the chain-dashed (closed squares) is data for ${ }^{158} \mathrm{Gd}$. 
sented for both ${ }^{158} \mathrm{Gd}$ (chain-dashed line, solid squares) and ${ }^{154} \mathrm{Gd}$ (dotted line, open squares). The transfer yields for both ${ }^{158} \mathrm{Gd}$ and ${ }^{154} \mathrm{Gd}$ are between the two calculated curves, the upper curve demonstrating a non-diabolic case, and the lower curve demonstrating the diabolic case. It should be noted, however, that the experimental data are not corrected for side-feeding, which could bave an effect on the final populations, and that the calculations were not done for the exact system studied experimentally. The precise position of the diabolical point depends in a sensitive way on the parameters of the model, such as deformation and pairing parameters. The present theories are not precise enough to predict the exact location of the diabolical point, and experiments may determine that it is shifted to a different neutron number from what is depicted in this case. Additionally, the statistics of the experiment were marginal (reflected in the error bars). We make no claim that these data show the SQUID effect, as the results lie between the calculatt d signatures, defying distinguishing between the two cases. However, it demonstrates the problems associated with theory-experiment comparisons and illustrates the quality of such comparisons. 


\section{$6.2{ }^{233} \mathrm{U}$ Results}

Figure 6.4 compares the rutational population pattern extracted from the University of Rochester (UOR) ${ }^{58} \mathrm{Ni}+{ }^{233} \mathrm{U}$ Coulomb excitation experiment with several theories. The experimentally observed pattern $(\bullet)$ is corrected for internal conversion and cascade-to-crossover ratios. Angular distribution effects are assumed to average out. Additionally, the maximum spin observed in the experiment is assumed to be the maximum state populated. As the beam energy for this experiment ( $249 \mathrm{MeV}$ ) was well below the Coulomb barrier (325 $\mathrm{MeV}$ ), no correction for side-feeding should be required. The population probability peaks around spin $\frac{19}{2}^{+}$and drops off to $\frac{1}{10}$ of the strength by spin $\frac{29}{2}^{+}$. The results of the Sudden Approximation methods of Section 5.1 are shown for the Monte Carlo method (dashed line, open circles) and the S-matrix method (dotted line, open squares) and generally reproduce the maximum population spin and fall off above the rainbow. The rainbow is a quantum mechanical effect where states higher than the maximum spin and classically forbidden are populated with an exponentially decreasing probability (or an upside-down rainbow). Both methods overestimate the location of the maximum probability, an indication that the Sudden Approximation may 


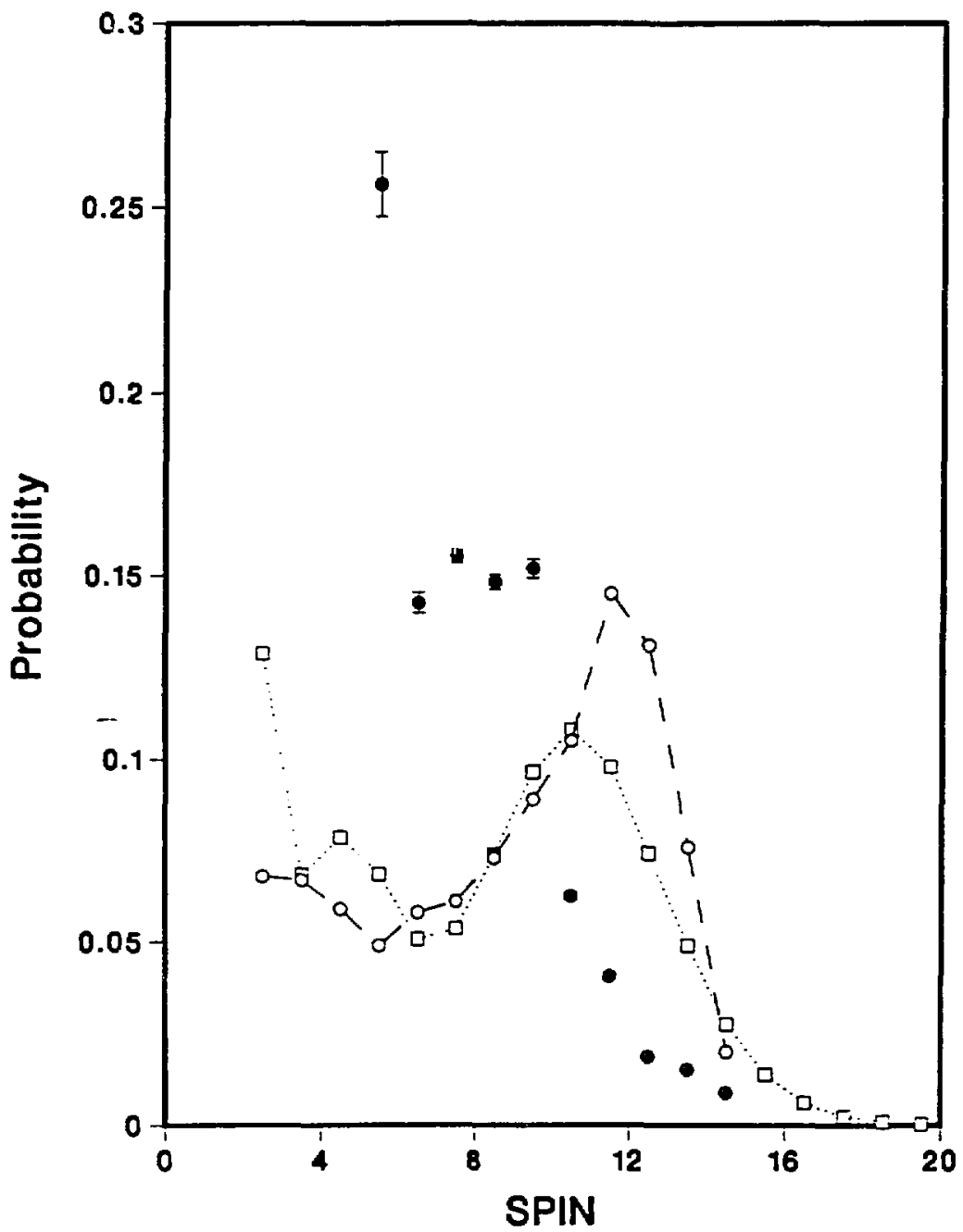

Figure 6.4: Rotational population pattern for the ${ }^{38} \mathrm{Ni}+{ }^{233} \mathrm{U}$ Coulomb eXcitation experiment. The experimental data $(\bullet)$ are compared to the Monte Carlo method (dashed line, open circles) and the S-Matrix method (dotted line, open squares). Note the oscillation around spin $\frac{11}{2}^{+}$in the calculation. 
be having some difficulties with systems as heavy as $\mathrm{Ni}+\mathrm{U}$. The location of the maximum probability can be decreased by 2-4 units of angular momentum with a $10 \%$ reduction in the quadrupole moment, $Q_{0}$. Two initial orientation angles, $\chi$, a large angle and a small angle, populate the same final spin, and thus, can quantum interfere. This interference pattern is known as Coulomb oscillations. The theoretical methods exhibit a Coulomb oscillation around spin $\frac{11}{2}^{+}$, in a region where experimental data doesn't exist. The Coulomb oscillations in the Monte Carlo method are more washed out, something that is observed in the ${ }^{206} \mathrm{~Pb}+{ }^{233} \mathrm{U}$ experiment also. However, such oscillations are not completely washed out in the experimental data. This, of course, generated the effort to look at other methods for calculating rotational population patterns. It should be noted that nuclear potential effects are not included in these calculations, probably not a serious approximation as the beam energy is below the Coulomb barrier. In general, these Sudden Approximation methods, while not predicting the overall magnitude of the probability correctly, seem to reproduce some of the oscillations in the rotational population pattern and roughly reproduce the location of the peak. Please note that the details of the location of the peak depend sensitively on the magnitude of the quadrupole moment and the beam energy. The drupole moment is not reduced by $10 \%$ 
in these calculations.

Figure 6.5 compares the rotational population pattern extracted from the SuperHILAC ${ }^{206} \mathrm{~Pb}+{ }^{233} \mathrm{U}$ inelastic excitation experiment with theory. The experimentally observed pattern $(\bullet)$ is plotted with error bars and is corrected for detector efficiency and internal conversion. The most noticeable feature of the probability pattern is the presence of the large oscillations at spins $\frac{13}{2}^{+}$, $\frac{19}{2}^{+}, \frac{29}{2}^{+}$and $\frac{37}{2}^{+}$, which appear to be due to Coulomb oscillations, or the interference pattern produced because two initial orientation angles $\chi$, a small angle and a large angle, yield the same final spin. It should be noted that the data are corrected for internal conversion and cascade-to-crossover ratios, but are not corrected for side-feeding. The maximum spin observed is assumed to be the highest spin populated in the experiment. Three theoretical calculations are presented, the Sudden Approximation methods (1) Monte Carlo (dashed curve, open circles), (2) S-matrix method (dotted curve, open square symbols) and (3) the Alder-Winther-deBoer method (chain-dashed line, closed squares). The Sudden Approximation methods have the quadrupole moment $Q_{0}$ reduced by $10 \%$ to compensate for the over-estimation of the maximum final spin populated, and generally reproduce the rainbow maximum. The rainbow maximum is not observed very strongly in the experimental data, 


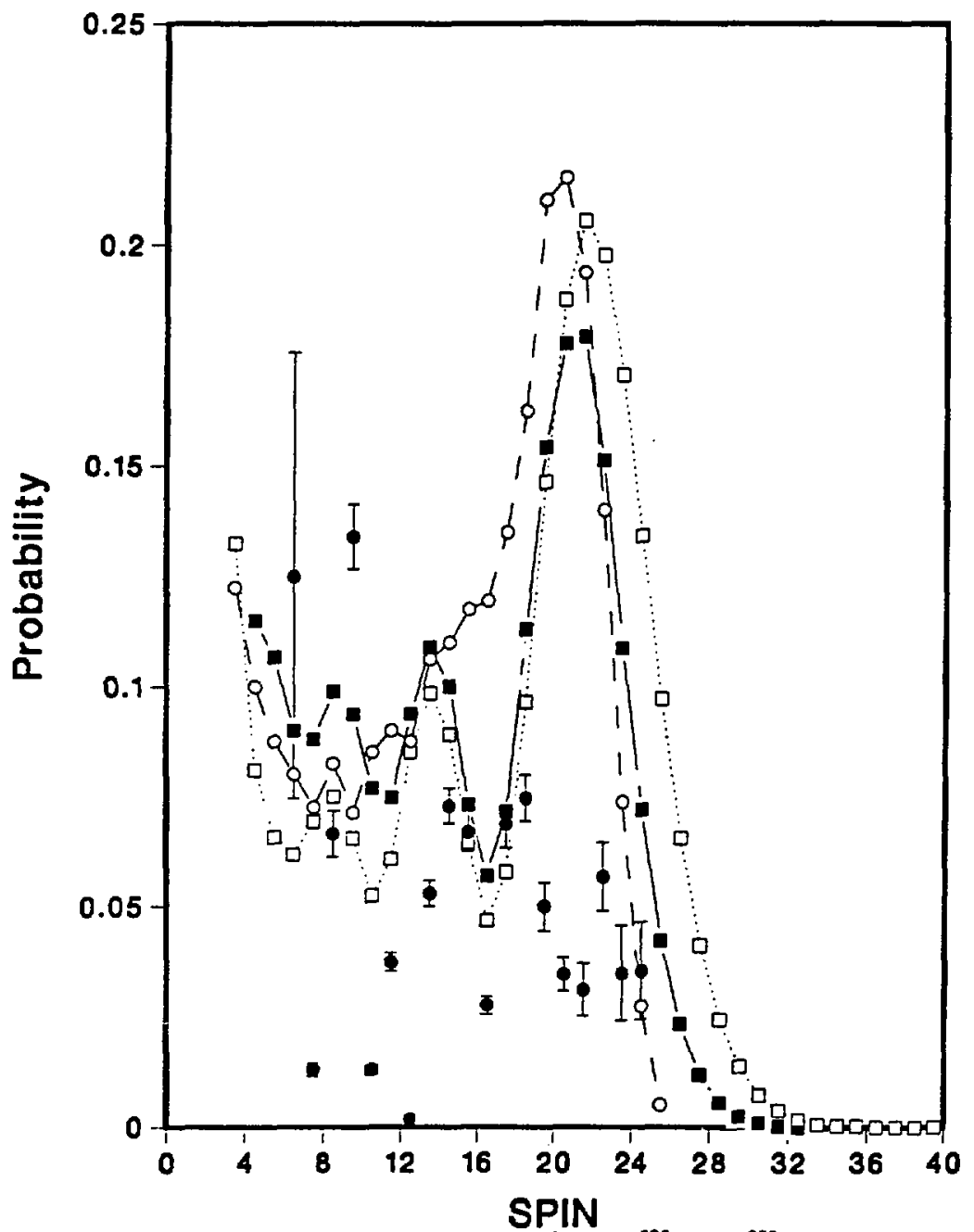

Figure 6.5: Rotational population pattern for the ${ }^{206} \mathrm{Fb}+{ }^{233} \mathrm{U}$ inelastic excitation experiment. Experimental data $(\bullet)$ are compared with the Monte Carlo method (dashed curve, open circles), S-Matrix method (dotted curve, open square symbols) and the Alder-Winther-deBoer method (chain-dashed line, closed squares). A complete discussion of this Figure is found in the text. 
consistent with the fact that the reaction is run with a beam energy near the Coulomb barrier, although the oscillation around spin $\frac{37}{2}^{+}$could be identified as such. The rainbow maximum was taken from the Alder-Winther-deBoer method calculations, and one can see that the Sudden Approximation methods (with $Q_{0}$ reduced by $10 \%$ ) generally agree. The Monte Carlo calculation seems to wash out the Coulomb oscillations at lower spins more than the other methods. The other two calculations agree very well on the location of those oscillations, around spins $\frac{17}{2}^{+}$and $\frac{27}{2}^{+}$. These compare very well, in quality if not in magnitude, to those oscillations observed in the experiment around spins $\frac{19}{2}^{+}$and $\frac{29}{2}^{+}$. However, it appears that the Alder-Winther-deBoer method offers a better comparison. Namely, a $10 \%$ reduction in $Q_{0}$ does not include dynamical effects which may be important in the final rotational population pattern (the data are not good enough to state whether this is the case or not) for such a heavy system. We have already seen this in the $\mathrm{Pb}+\mathrm{Dy}$ calculations. The target is not stationary during the collision, and especially for large initial orientation $\chi$ angles $\left(>60-70^{\circ}\right)$, the nucleus may rotate past $90^{\circ}$ during the collision, changing the sign of the torque which may actually reduce the final spin populated. Overall, the comparison of theory with experimental data is marginal. 
scaled up by the conservation laws of angular momentum and magnetic flux. The field is typically scaled to $10^{12}$ gauss. There is other evidence of such strong fields. The remnant of the crab supernova is still accelerating with an apparent energy input of $\sim 10^{38} \mathrm{ergs} / \mathrm{s}$, and the most likely source of input energy is the absorption of magnetic dipole radiation from the fast pulsar within it. Energy balance implies about the same strength for the magnetic field as quoted above,

$$
10^{38} \mathrm{ergs} / \mathrm{s}=-\frac{d E}{d t}=-\frac{d}{d t}\left(\frac{1}{2} I \omega^{2}\right)=\frac{1}{6} R^{6} B^{2} \omega^{4} \sin ^{2} \alpha
$$

Given the observed period and rate of change of period,

$$
P \sim \frac{1}{30} \mathrm{~s}, \quad \dot{P} \sim 4 \times 10^{-13} \mathrm{~s} / \mathrm{s}
$$

we find (taking $\sin \alpha=1$ ),

$$
\begin{aligned}
B \sim 4 \times 10^{12} \text { gauss, } & I \sim 2 \times 10^{4.4} \mathrm{~g} \mathrm{~cm}^{2} \\
E_{\text {rot }} & \sim \frac{1}{2} I \omega^{2} \sim 4 \times 10^{48} \mathrm{ergs}
\end{aligned}
$$

where I use

$$
3.5 \times 10^{24} \text { gauss } \mathrm{cm}=1
$$

in gravitational units. The field will in general be oriented in a different direction than the rotation axis, say by an angle $\alpha$. It is believed, but not understood, that radiation over a broad band of frequencies is emitted within some angular spread along the magnetic axis $[\overline{7}, 8]$. Given the rotation, one has a beacon which we see as pulses as the star rotates.

We can use the period of rotation to estimate an average energy density of a millisecond pulsar. For the star to hold together under the opposing forces of gravity and centrifuge, we must have,

$$
\alpha^{2} \frac{G m M}{R^{2}}>m \Omega^{2} R
$$

Hence for the average density,

$$
\bar{\epsilon}=M /\left(\frac{4 \pi}{3} R^{3}\right)>\frac{3 \pi}{(\alpha P)^{2}}=3.3 \times 10^{14} \mathrm{~g} / \mathrm{cm}^{3}
$$

where the last equality holds for a millisecond pulsar. The factor $\alpha$ is unity in Newtonian physics and is empirically found to be about 0.65 in General relativity [9]. Since $\epsilon_{0} \approx 2.5 \times 10^{14} \mathrm{~g} / \mathrm{cm}^{3}$, we learn that the average density of a pulsar has to be about as large or larger than nuclear density and se pulsars must be neutron stars. In actual models that I have studied, $\bar{\rho} / \rho_{0}$ ranges from 3 for stiff equations of state to 5 for 


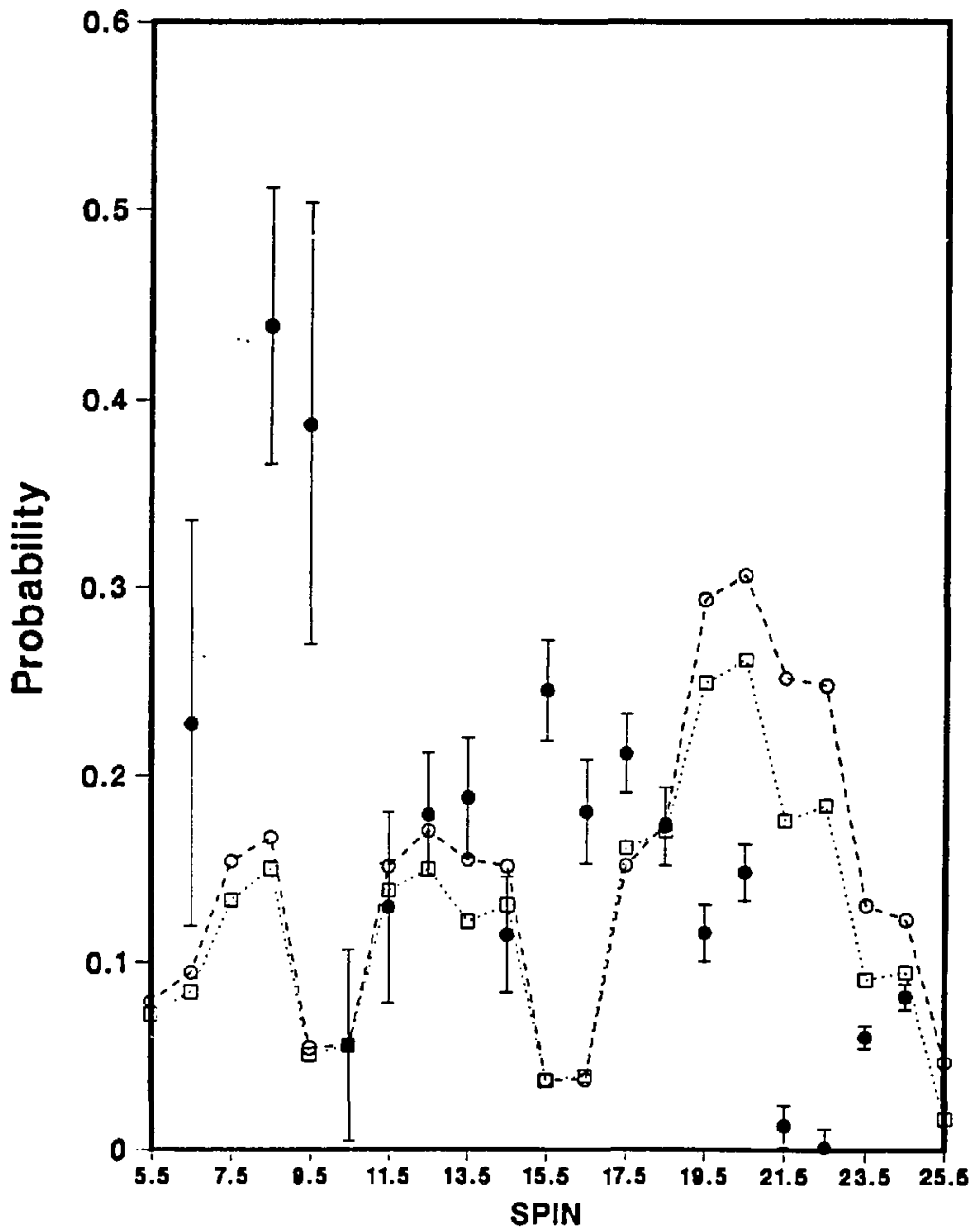

Figure 6.6: Inelastic excitation rotational population pattern in the ground band of ${ }^{239} \mathrm{Pu}$. Experimental data $(\bullet)$ are compared with Alder-WintherdeBoer theories. Pure Coulomb excitation (dashed line, open circles) for ${ }^{239} \mathrm{Pu}$ is compared to a calculation (dotted line, open squares) using ${ }^{238} \mathrm{Pu}$ energy levels and partitioning final probabilities as discussed in the text. As the two theories are very similar, the approximation of a spectator odd-neutron is valid. The theory curves are normalized to the experimental data at $\operatorname{spin} \frac{21}{2}^{+}$. 
spins than are experimentally observed, although the dip around spin $\frac{21}{2}^{+}$is qualitatively reproduced. The dip at spin $\frac{29}{2}^{+}$in the data may qualitatively be associated with the dip in the theories around spin $\frac{31}{2}^{+}$; however, the dip at $\frac{45}{2}^{+}$is not reproduced. If there was population of states higher than $\frac{49}{2}^{+}$ in the experiment, the last two points may be higher than normal, as some transition strength (for the higher transitions) would not be subtracted out correctly.

Figure 6.7 presents the rotational population pattern derived from spot intensities in the $\gamma \cdot \gamma$ coincidence spectrum gated on Yrast transitions. The experimental errors are larger because of poorer statistics. Again, the population probabilities are corrected for internal conversion, and I assume the $\frac{11}{2}^{+}$ level is the highest populated in the reaction and neglect cascade-to-crossover ratios. Relative spot intensities are extracted from the $\gamma-\gamma$ coincidence spectrum by gating on individual rotational transition $\gamma$-rays for ${ }^{239} \mathrm{Pu}$ and then fitting the resulting spectrum with peaks of fixed energy and width. The peak positions and widths were obtained from fits of Figure 3.17. The Gaussian width used was $2.9 \mathrm{keV}$. The relative spot intensities are tabulated in Table 6.1 and are corrected for detector efficiency and internal conversion. 


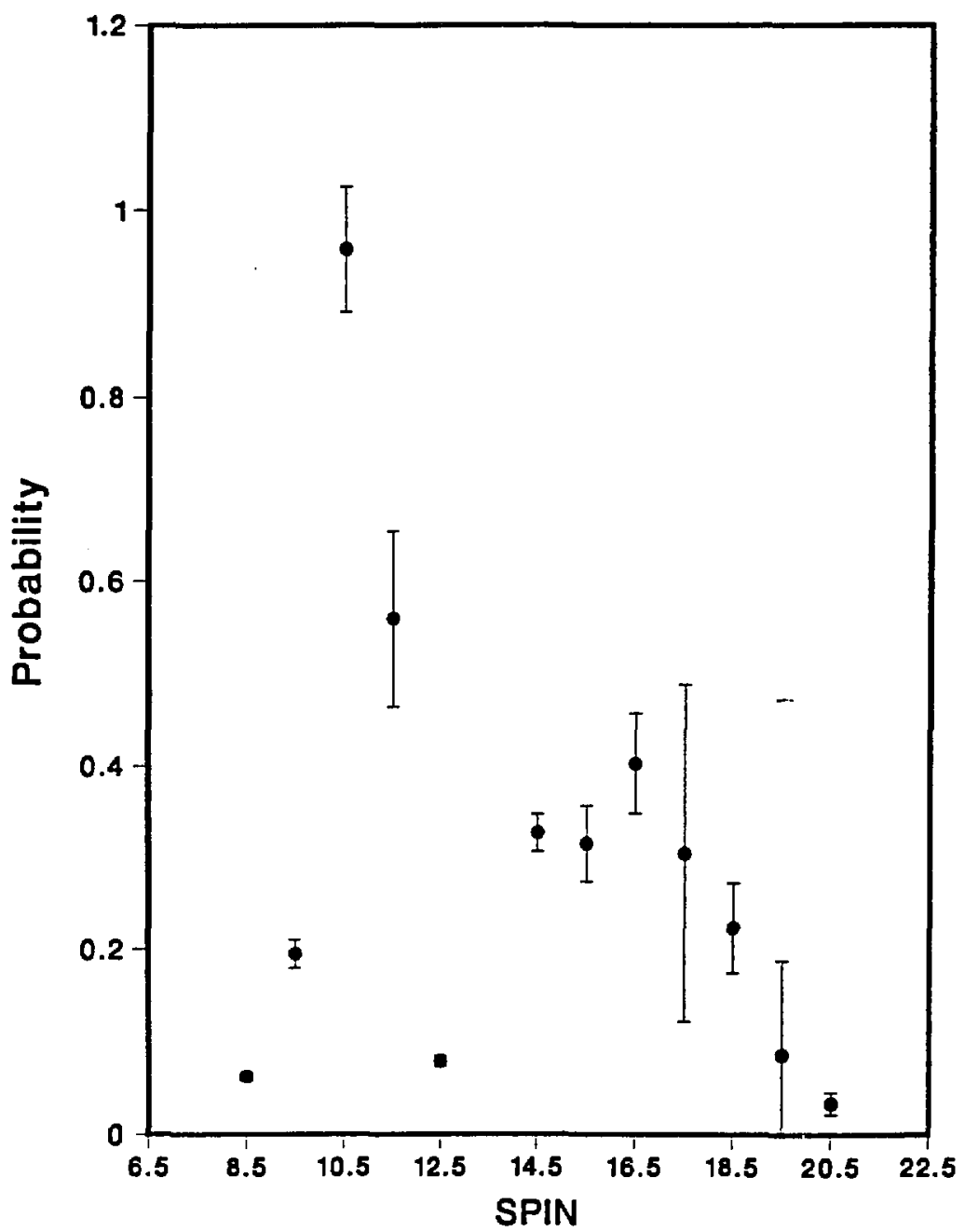

Figure 6.7: Inelastic rotational population pattern for the ground band in ${ }^{239} \mathrm{Pu}$ extracted from the $\gamma-\gamma$ coincidence spectrum gated on Y'rast transitions. 
Table 6.1: Relative spot intensities for the probability of populating a given spin in ${ }^{239} \mathrm{Pu}$ including a gate to exclude side-feeding.

\begin{tabular}{|c|c|c|c|c|}
\hline Trans. (keV) & 154.0 & 165.0 & 201.1 & 211.8 \\
\hline 154.0 & - & - & $276011 \pm 15 \%$ & $94704 \pm 42 \%$ \\
165.0 & - & - & $24336 \pm 138 \%$ & $166513 \pm 19 \%$ \\
201.1 & $226192 \pm 19 \%$ & - & - & - \\
211.8 & - & $153533 \pm 20 \%$ & - & - \\
245.3 & $122377 \pm 28 \%$ & $61300 \pm 44 \%$ & $308315 \pm 6 \%$ & $24245 \pm 65 \%$ \\
256.7 & - & $97902 \pm 27 \%$ & $24557 \pm 68 \%$ & $164261 \pm 10 \%$ \\
288.1 & - & $32825 \pm 82 \%$ & $150574 \pm 11 \%$ & $45546 \pm 35 \%$ \\
239.6 & - & $29506 \pm 92 \%$ & $10462 \pm 163 \%$ & $90297 \pm 15 \%$ \\
328.5 & $69283 \pm 51 \%$ & - & $100196 \pm 18 \%$ & $16523 \pm 98 \%$ \\
340.0 & - & $38120 \pm 72 \%$ & $28752 \pm 61 \%$ & $66602 \pm 25 \%$ \\
367.3 & $142833 \pm 26 \%$ & - & $100885 \pm 18 \%$ & - \\
379.0 & - & - & $22726 \pm 82 \%$ & $30474 \pm 57 \%$ \\
403.5 & - & $24161 \pm 122 \%$ & $45710 \pm 42 \%$ & $16005 \pm 112 \%$ \\
415.5 & - & - & - & - \\
437.8 & $53319 \pm 83 \%$ & - & - & - \\
450.9 & - & - & - & - \\
\hline
\end{tabular}


Table 6.1: Continuation of relative spot intensities for ${ }^{239} \mathrm{Pu}$

\begin{tabular}{|c|c|c|c|c|}
\hline Trans. (keV) & 245.3 & 256.7 & 288.1 & 299.6 \\
\hline 154.0 & $145911 \pm 23 \%$ & $56788 \pm 60 \%$ & $18944 \pm 176 \%$ & - \\
165.0 & $52385 \pm 51 \%$ & $130946 \pm 21 \%$ & - & - \\
201.1 & $252237 \pm 7 \%$ & $39857 \pm 42 \%$ & $143931 \pm 12 \%$ & - \\
211.8 & $10717 \pm 143 \%$ & $125168 \pm 13 \%$ & $20928 \pm 74 \%$ & $59265 \pm 27 \%$ \\
245.3 & - & - & $84728 \pm 16 \%$ & - \\
256.7 & - & - & - & $51651 \pm 27 \%$ \\
288.1 & $87704 \pm 16 \%$ & - & - & - \\
299.6 & - & $87588 \pm 16 \%$ & $10686 \pm 130 \%$ & - \\
328.5 & $87490 \pm 16 \%$ & $21484 \pm 8 \%$ & $170677 \pm 8 \%$ & - \\
340.0 & $17199 \pm 80 \%$ & $134002 \pm 11 \%$ & - & $76425 \pm 19 \%$ \\
367.3 & $8789 i \pm 17 \%$ & - & $81335 \pm 18 \%$ & - \\
379.0 & - & $10347 \pm 143 \%$ & - & $66766 \pm 24 \%$ \\
403.5 & $39141 \pm 40 \%$ & $13677 \pm 113 \%$ & $12900 \pm 119 \%$ & - \\
415.5 & - & - & - & - \\
437.8 & $72610 \pm 23 \%$ & - & $19967 \pm 91 \%$ & - \\
450.9 & - & - & - & - \\
\hline
\end{tabular}


Table 6.1: Continuation of relative spot intensities for ${ }^{239} \mathrm{Pu}$

\begin{tabular}{|c|c|c|c|c|}
\hline Trans. (keV) & 328.5 & 340.0 & 367.3 & $379 . n$ \\
\hline 154.0 & $102826 \pm 34 \%$ & - & $102070 \pm 36 \%$ & - \\
165.0 & $20355 \pm 134 \%$ & $36393 \pm 75 \%$ & - & - \\
201.1 & $31101 \pm 56 \%$ & $27263 \pm 63 \%$ & $71385 \pm 25 \%$ & - \\
211.8 & $30862 \pm 52 \%$ & $69587 \pm 23 \%$ & $16278 \pm 101 \%$ & $3271 \pm 52 \%$ \\
245.3 & $79077 \pm 17 \%$ & $16763 \pm 82 \%$ & $60478 \pm 24 \%$ & $10920 \pm 132 \%$ \\
256.7 & - & $121123 \pm 12 \%$ & - & $22697 \pm 64 \%$ \\
288.1 & $150486 \pm 9 \%$ & - & $64482 \pm 23 \%$ & - \\
299.6 & - & $59691 \pm 93 \%$ & $7670 \pm 192 \%$ & $7262 \pm 22 \%$ \\
328.5 & - & $15762 \pm 93 \%$ & $84585 \pm 18 \%$ & - \\
340.0 & - & - & - & $69662 \pm 22 \%$ \\
367.3 & $72072 \pm 21 \%$ & - & - & - \\
379.0 & - & $49222 \pm 31 \%$ & - & - \\
403.5 & - & - & $39410 \pm 42 \%$ & - \\
415.5 & - & - & - & - \\
437.8 & - & - & $19938 \pm 92 \%$ & $10225 \pm 181 \%$ \\
450.9 & - & - & - & - \\
\hline
\end{tabular}


Table 6.1: Continuation of relative spot intensities for ${ }^{239} \mathrm{Pu}$

\begin{tabular}{|c|c|c|c|c|}
\hline Trans. (keV) & 403.5 & 415.5 & 437.8 & 450.9 \\
\hline 154.0 & - & - & - & $23354 \pm 180 \%$ \\
165.0 & - & - & - & - \\
201.1 & $16118 \pm 119 \%$ & - & - & - \\
211.8 & $24404 \pm 72 \%$ & $12883 \pm 137 \%$ & - & - \\
245.3 & $31882 \pm 47 \%$ & - & $46828 \pm 35 \%$ & - \\
256.7 & - & - & - & $32949 \pm 52 \%$ \\
288.1 & $25596 \pm 60 \%$ & - & $9605 \pm 179 \%$ & - \\
299.6 & - & - & - & - \\
328.5 & - & - & - & - \\
340.0 & - & $8275 \pm 191 \%$ & - & - \\
367.3 & - & - & - & - \\
379.0 & - & - & - & $15567 \pm 124 \%$ \\
403.5 & - & - & $25088 \pm 68 \%$ & - \\
415.5 & - & - & - & - \\
437.8 & - & - & - & - \\
450.9 & - & - & - & - \\
\hline
\end{tabular}


Figure 6.8 shows a calculation of ${ }^{239} \mathrm{Pu}$ rotational population patterns using the methods of Section 5.2.3. The large amount of population probability at lower spins is unexplained with theory. The absorption from the nuclear potential completely suppresses population of high spins, which may be enhancing the peak at lower spins.

\subsection{3 $\quad{ }^{238} \mathrm{Pu}$ One-Neutron Transfer Results}

Figure 6.9 shows the rotational population patterns for rotational states populated in the one-neutron transfer reaction ${ }^{90} \mathrm{Zr}+{ }^{239} \mathrm{Pu} \rightarrow{ }^{91} \mathrm{Zr}+{ }^{238} \mathrm{Pu}$ for beam encrgy $500 \mathrm{MeV}$ and mean scattering angle of $140^{\circ}$ in the lab. The closed circles $(\bullet)$ are for non-Yrast gated events corsected for internal conversion and detector efficiency, and the closed squares are for Yrast gated events also corrected for internal conversion and detector efficiency. In both cases, the highest transition populated was assumed to be the highest transition shown in the Figurc. The relative spot intensities from the $\gamma-\gamma$ coincidence spectrum are shown in Table 6.2. The spot intensities are obtained as described in Section 6.3.1, and are corrected for detector efficiency and internal conversion. The points at spin 6 and 8 are questionable because the corrections to the 


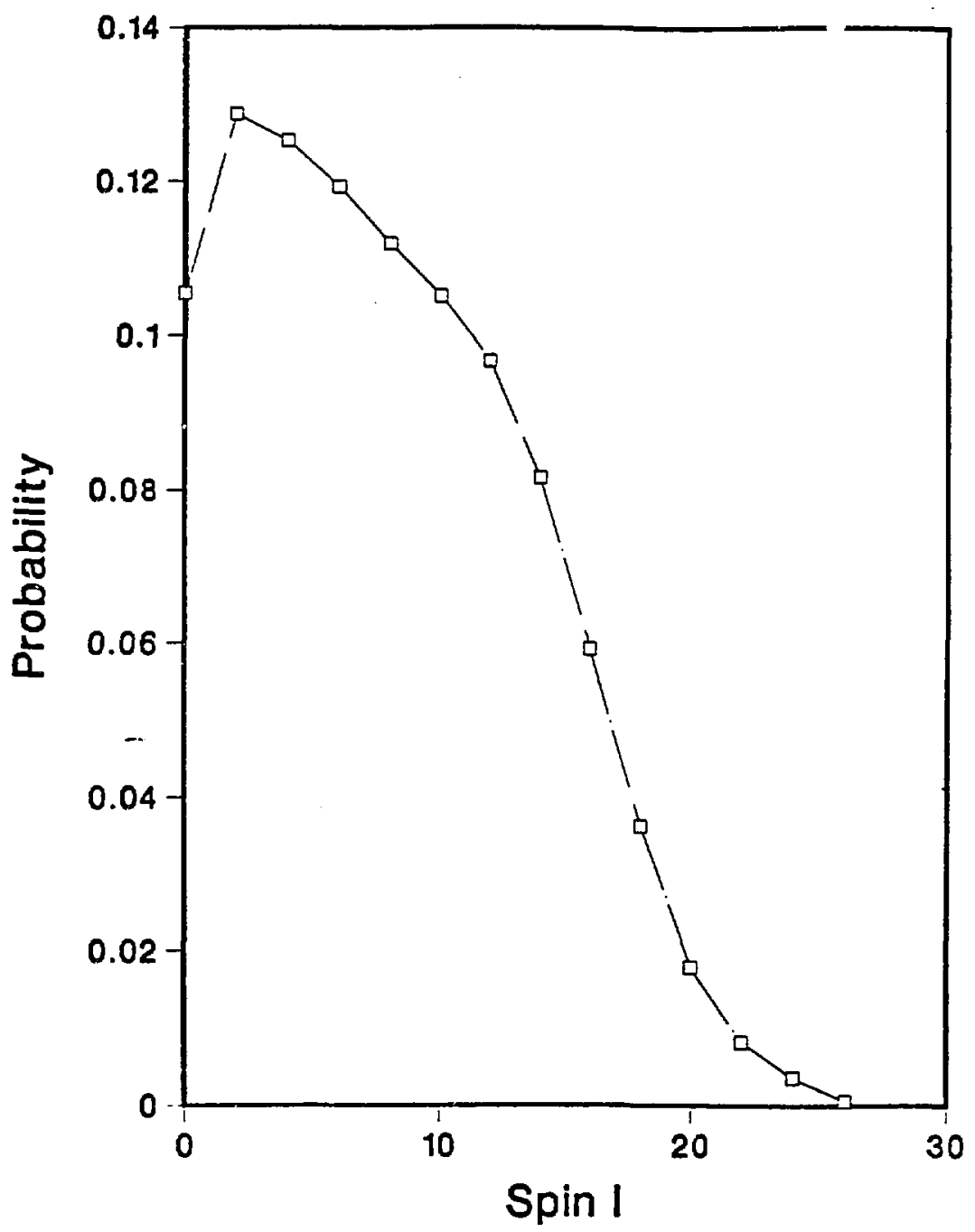

Figure 6.8: Theoretical calculation of rotational population probability for the inelastic excitation experiment ${ }^{90} \mathrm{Zr}+{ }^{239} \mathrm{Pu}$. 




Figure 6.9: Rotational population pattern for the states populated in ${ }^{238} \mathrm{Pu}$ in the one-neutron transfer reaction. Both the probabilities of populating a certain spin excluding side-feeding (closed squares) and including side-feeding (•) are shown. The data are normalized at spin 12. The negative points arise from the subtraction of transition intensities in experiment. Their errors are consistent with the probability being equal to zero. 
Table 6.2: Relative spot intensities for the probability of populating a given spin in ${ }^{238} \mathrm{Pu}$ including a gate to exclude side-feeding.

\begin{tabular}{|c|c|c|c|c|}
\hline Trans. $(\mathrm{keV})$ & 157.2 & 210.0 & 259.7 & 306.5 \\
\hline 157.2 & - & $44285 \pm 107 \%$ & $43283 \pm 72 \%$ & $47523 \pm 84 \%$ \\
210.0 & $76842 \pm 46 \%$ & - & $66557 \pm 35 \%$ & $48594 \pm 45 \%$ \\
259.7 & $45906 \pm 62 \%$ & $105791 \pm 28 \%$ & - & $31494 \pm 53 \%$ \\
306.5 & $73321 \pm 41 \%$ & $50770 \pm 44 \%$ & $41001 \pm 45 \%$ & - \\
348.9 & - & - & - & $17446 \pm 82 \%$ \\
389.5 & $22852 \pm 142 \%$ & $17903 \pm 113 \%$ & $33912 \pm 63 \%$ & $28988 \pm 55 \%$ \\
426.3 & - & - & $8458 \pm 184 \%$ & $25539 \pm 72 \%$ \\
\hline Trans. (keV) & 348.9 & 389.5 & 426.3 & - \\
\hline 157.2 & - & - & - & - \\
210.0 & - & $14648 \pm 128 \%$ & - & - \\
259.7 & $18056 \pm 93 \%$ & $34002 \pm 56 \%$ & - & - \\
306.5 & $41122 \pm 44 \%$ & $35890 \pm 54 \%$ & $30647 \pm 56 \%$ & - \\
348.9 & - & - & - & - \\
389.5 & $13282 \pm 119 \%$ & - & - & - \\
426.3 & $11825 \pm 137 \%$ & $18011 \pm 127 \%$ & - & - \\
\hline
\end{tabular}

data become very large at these low spins and the errors are correspondingly larger. Thus, the predominant fcature of the data is the dip in population probability at spin 14 .

Several different theoretical calculations are shown in Figures 6.10,6.11 and 6.12. Figure 6.10 shows pure Coulomb excitation (chain-dashed line, closed squares) for comparison. The solid line (•) is an Alder-Winther-deBoer calculation in which the real part of the nuclear potential is included in the coupling, the imaginary part is included in the $a_{a b s}$ term in the transfer matrix $T_{l^{\prime \prime}}$, and 




Figure 6.10: Several theoretical calculations of the rotational population patterns in ${ }^{238} \mathrm{Pu}$ from one-neutron transfer using deformation $\eta=6$. Pure Coulomb excitation (chaindashed line, solid squares) is shown for comparison. Both the spin-up part of the wavefunction (dotted line, open circles) and the spin-down part of the wavefunction (dashed line, open squares) are individually plotted for reasons given in the text. The calculation including the total wavefunction is shown in the solid line (•). 


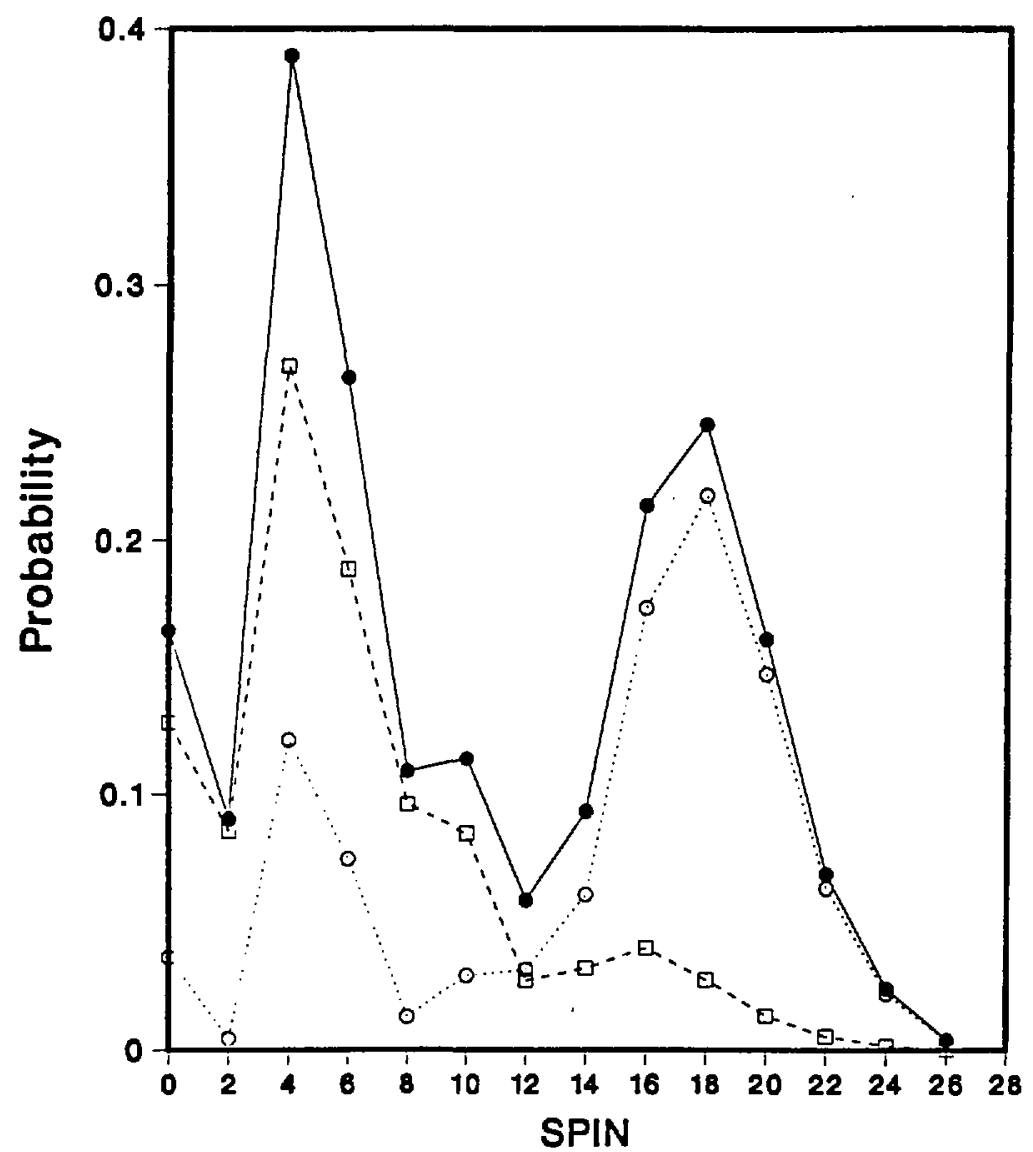

Figure 6.11: Same as Figure 6.10 but for delormation $\eta=4$. Pure Coulomb excitation is not shown in this figure. 


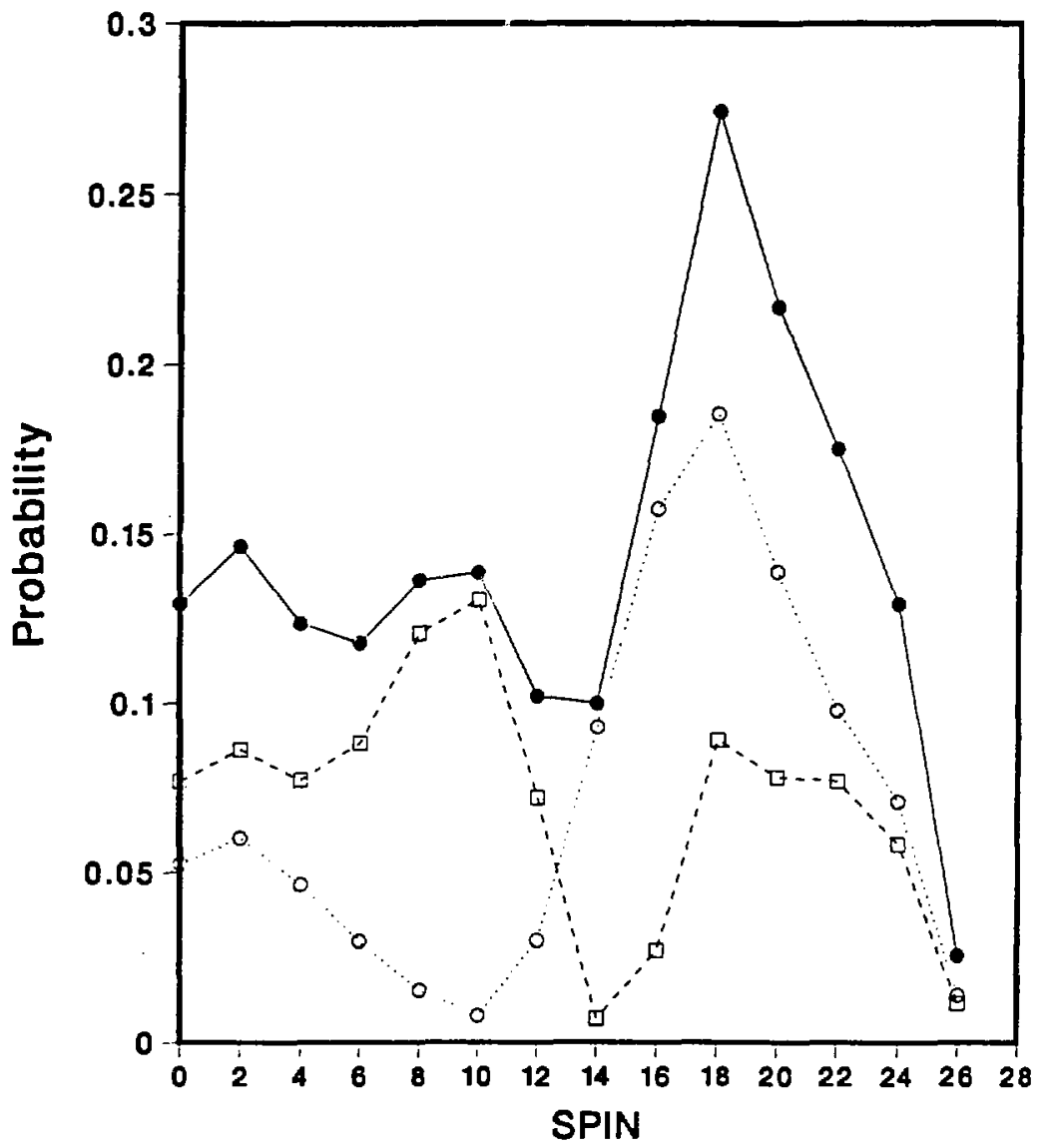

Figure 6.12: Best theoretical calculation of the rotational population patterns in ${ }^{238} \mathrm{Pu}$ from one-neutron transfer. As discussed in the text, the radial part of the ravefunction is now included in the form factor. The symbols are the same as in Figure 6.11. 
$a_{\text {tun }}$ factor is included in the transfer matrix $T_{I^{\prime} l}$, effective $Z$ and $E_{\text {beam }}$ are used to reproduce the correct turning point and force at the distance of closest approach for $140^{\circ}$ scattering in the lab, centrifugal barrier effects are included in the $T_{f^{\prime} l}$, the odd-neutron is considered as a spectator, and the Nilsson wavefunction for the odd-neutron in ${ }^{239} \mathrm{Pu}$ is included in the surface form factor in the transfer matrix. The spin-up (dotted line, open circles) and spin-down (dashed line, open squares) parts of the wavefunction are included separately, and the probabilities are shown individually for those cases, as in principle, polarized ${ }^{239} \mathrm{Pu}$ target experiments could be performed to investigate each of those population probability patterns. The wavefunctions used were:

$$
\Psi_{\text {up }}=1.0 Y_{60}+0.979 Y_{40}-2.021 Y_{20}-1.712 Y_{00}
$$

and

$$
\Psi_{\text {doun }}=-2.746 Y_{61}+2.621 Y_{41}+2.034 Y_{21}
$$

where the Y's are pherical harmonics and the coefficients are from Nilsson's old tabulations [9.1] for diagonalization within one oscillator shell $(N=6)$. The results in Figure 6.10 are for deformation $\eta=6$, slightly larger than the experimental deformation of ${ }^{239} \mathrm{Pu}$. Calculations for $\eta=4$ (slightly smaller than the actual deformation) were also performed and are shown in sigure 6.11, 
with no significant difference between $\eta=6$ and $\eta=4$. The solid line is the total probability (sum of spin-up and spin-down probabilities). As observed in Figure 6.10, the spin-up part of the wavefunction emphasizes population of higher spins, while the spin-down part of the wavefunction emphasizes the lower spins. Additionally, there is this dip in probability at spin 14.

Figure 6.12 shows the results of a more complete calculation where the radial parts of the wavefunction are also included in the angular surface form factor in the transfer matrix. Namely, the wavefunctions become:

$$
\Psi_{\text {up }}=1.0 R_{6} Y_{60}+0.879 R_{4} Y_{40}-2.021 R_{2} Y_{20}-\overline{1} .712 R_{0} Y_{00}
$$

and

$$
\Psi_{\text {down }}=-2.746 R_{6} Y_{61}+2.621 R_{4} Y_{41}+2.034 R_{2} Y_{21}
$$

where the $R_{L}$ functions are the sixth oscillator shell radial wavefunctions evaluated at the turning radius for the s-state $(9.8 \mathrm{fm})$. This is approximately the distance of closest approach for the reaction being considered. These radial wavefunctions were calculated numerically by the "shooting method" in a spherical Woods-Saxon potential of size appropriate to ${ }^{239} \mathrm{Pu}$ but withrut spin-orbit coupling. These radial wavefunctions $R_{L}(9.8 \mathrm{fm})$ are $0.248,0.216$, 0.154 and 0.075 for $L=0,2,4$ and 6 , respectively. 
In addition, the centrifugal factor (see Section 5.2.4) is removed, as it is more naturally included in the more complete wavefunctions, and the $a_{t u n}$ factor is set to unity. The Nilsson single oscillator shell expansion is best interpreted as an expansion on a spheroid of half the eccentricity of the deformed constant density nuclear surface. The classical turning surface is also prolate due to the electric quadrupole potential. Thus, the tunnelling distance will be nearly constant. Figure 6.13 shows the $a_{a b s}$ factor and the spin-up and spin-down parts of the surface form factor as a function of $\cos (\chi)$. As can be seen in Figure 6.13, both the spin-up and spin-down form factors have a node, and they are slightly out of phase with each other. The $a_{a b}$ factor decreases sharply for small orientation angles as expected.

As can be seen in Figure 6.12, again the spin-up part of the wavefunction emphasizes the higher spins and the spin-down part emphasizes lower spins. Additionally, the dip around spin 14 is still observed. Figure 6.14 compares the experimental probabilities with the most complete theoretical calculation, normalized at spin 10 . While the magnitude of the experimental minimum is not reproduced, the calculation does qualitatively match the experimental data. Indeed, this dip is fairly robust in all of the calculations performed so far and appears to qualitatively match the experimental data. This minimum 




Figure 6.13: Parts of the surface form factor $F_{\left.l^{\prime}\right]}$ for ${ }^{239} \mathrm{Pu}$. The chain-dashed line is the $a_{a b s}(x)$ term, the dotted line is the spin-up part of the wavefunction times the absorption factor, and the dashed line is the spin-down part of the wavefunction times the absorption factor. 


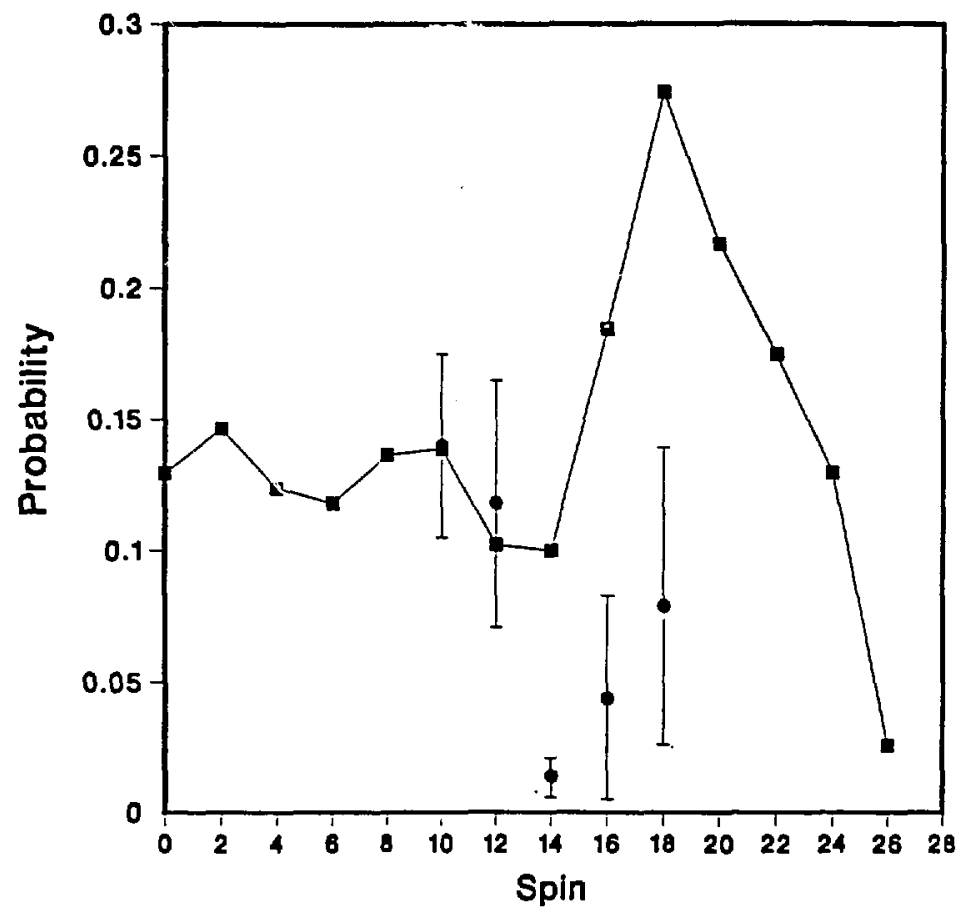

Figure 6.14: Comparison of the best theoretical calculation of the rotational population patterns in ${ }^{238} \mathrm{Pu}_{\mathrm{u}}$ from one-neutron transfer wich experimental data. 
probability could be a result of a left-over Coulomb oscillation or could also be due to the different regions of spin emphasized by the spin-up and spindown parts of the wavefunctions used in the transfer matrix. Nevertheless, this is the first comparison of experimental and theoretical rotational population patterns in one-neutron transfer to ${ }^{233} \mathrm{Pu}$.

Several extensions can be made to the theoretical calculations: (1) the structure of the prcjectile could be included, (2) transfer at all distances along the Rutherford trajectory could be included, (3) the Alder-Winther-deBoer method could be generalized to other scattering angles $(M \neq 0$ terms included), and (4) a rectangular transfer matrix could be included rather than the square matrix used in the calculations described above; namely, rotational states in ${ }^{239} \mathrm{Pu}$ are Coulomb excited on the inward path, a transfer matrix linking the half-integer odd-A rotation al states in ${ }^{239} \mathrm{Pu}$ and even-integer even-even rotational states in ${ }^{238} \mathrm{Pu}$ is used at closest approach, and then the states in ${ }^{238} \mathrm{Pu}$ are Coulomb excited on the outward path. However, it is anticipated that the general features of the rotational population pattern are captured in the calculations already presented above. 


\section{Chapter 7}

\section{Conclusions}

This thesis has presented data on the ground-state rotational bands in ${ }^{23} \mathrm{U}$, ${ }^{234} \mathrm{U},{ }^{235} \mathrm{U},{ }^{238} \mathrm{Pu}$ and ${ }^{239} \mathrm{Pu}$ from heavy-ion Coulomb excitation, inelastic excitation and one-neutron transfer reactions. Knowledge of high-spin states in the actinide region has been significantly enhanced, with spin states as high as $26^{+}$observed. A large amount of spectroscopic information (moments-ofinertia, Routhians, aligned angular mome, tum, $\mathrm{g}$-factors, etc.) was presented. The one-neutron transfer reaction was proven to be useful in exploring highspin states in the actinide region because the rather "cold" transfer mechanism suppresses fission (see Section 3.1).

Inelastic excitation and Coulomb excitation reactions were used to populate states in the ground rotational band of ${ }^{233} \mathrm{U}$ (see Section 3.2). The momentsof-inertia indicated no sharp backbending, but at high spins a possible soft 
band crossing was observed. In addition, at high spins a reversal in the sense of the staggering between the favored and unfavored signatures of the groundband was noted. Band fitting of the ground-band in ${ }^{233} \mathrm{U}$ proved to be very difficult. A trend in the g-factors of some of the lower spin states supported the mounting evidence of a change in the character of the ground-band with increasing angular momentum. Cranking calculations were performed. It was discovered that a slight $\gamma$-deformation or triaxiality best explained both the band crossing and the reversal of the staggering in the ground rorational band of ${ }^{233} \mathrm{U}$.

Data were also presented for ${ }^{238} \mathrm{Pu}$ and ${ }^{239} \mathrm{Pu}$ ground rotational bands (see Section 3.3). We observed no sharp backbending in either nucleus, although a band crossing could not be ruled out. The probability of neutron transfer as a function of distance of closest approach could be explained by a simple barrier penetration model; however, an anomalous $H, K$ spectrum indicated that transfer of the odd-neutron in the $\frac{1}{2}^{+}[631]$ orbital was dominant, and tnat transfer of a neutron from a lower Nilsson orbit, thus breaking a pair, to form a tivo quasi-particle state was not observed. This indicates the importance of nuclear structure in the transfer process.

Fo: the first time, theoretical calculations of rolational population patterns 
for actinide nuclei were compared with experimental data. The importance of including nuclear potential effects, dynamic effects, and nuclear structure effects was highlighted and discussed. The most reasonable agreement between theory and experiment was obtained with a modified Alder-Winther-deBoer method which included a transfer matrix at closest approach. Qualitative agreement between theory and the data was obtained, although a lot more theoretical work is needed before : complete understanding of how the oneneutron transfer process populates high-spin states is possible.

Several possible future experiments can be proposed. The two-neutron transfer reaction ${ }^{240} \mathrm{Pu}(A, A+2){ }^{238} \mathrm{Pu}$ would be interesting to study, to perhaps measure enhancement factors for two-neutron transfer at large distances of approach in the actinide region for the first time. Additionally, a oneneutron transfer experiment ${ }^{239} \mathrm{Pu}\left({ }^{117} \mathrm{Sn},{ }^{118} \mathrm{Sn}\right){ }^{238} \mathrm{Pu}$ has been proposed [95] to attempi to populate and observe transitions of a rotational band in shape isomeric states of ${ }^{238} \mathrm{Pu}$. Lastly, other projectile-target combinations in the actinide region are desirable to study high-spin states and to expand the number of rotational bands in the region. The availability of radioactive nuclear beams also raises the possibility of neutron stripping reactions, and greatly enlarges the number of nuclei that could be studied. For instance, at high 
spins, because Coriolis and centrifugal forces affect the high- $j$ orbitals more as the cranking frequency increases, perhaps the super-heavy $h \frac{11}{2}$ or $k \frac{17}{2}$ neutron orbitals could be discovered in ${ }^{249} \mathrm{Cm}$ from the ${ }^{248} \mathrm{Cm}(A, A-1){ }^{5} \mathrm{Cm}$ onereutron transfer reaction. This, of course, would help to locate those orbitals and place restrictions on models for calculating the half-lives of super-heavy elements.

Devices such as GAMMASPHERE could greatly expand the nuclear s: :ucture studies that can be done. With a tool that allows one to look at sry specific higher-fold $\gamma$-coincidences, rotational bands can be studied in intricate detail. For instance, investigation of superdeformed bands that contain $\sim 1 \%$ of the strength of the ground bands discussed in this thesis becomes fairly easy.

In conclusion, this thesis presents data and theoretical calculations to attempt to interpret the data for several nuclei in the actinide region. At least a qualitative understanding of the reactions studied is presented. The future, however. portends many new experiments to study rotational levels in the actinide region. 


\section{Appendix A}

\section{Target Construction}

\section{A.1 General Apparatus}

Target-making is truly an area in which science and art collide, for sometimes just following the formula was not enough to obtain a satisfactory target. The ${ }^{233} \mathrm{U}$ and ${ }^{239} \mathrm{Pu}$ targets used in this work were made using the electrodeposition method. As the target material was radioactive (mostly $\alpha$ decay, with some $\gamma$-rays and spontaneous fission), all operations were confined to glove boxes for safety purposes. Figure A.1 shows a side view of the electrodeposition apparatus, which consisted of a stainless-steel base-plate cathode to which the target backing is attached, glass chimney $6 \mathrm{~mm}$ in diameter, $\mathrm{Pt}$ wire anode, and electroplating solution. The target backing is spot welded to an $\mathrm{Al}$ target holder, which is then fastened with screws to the base-plate (in the Be 


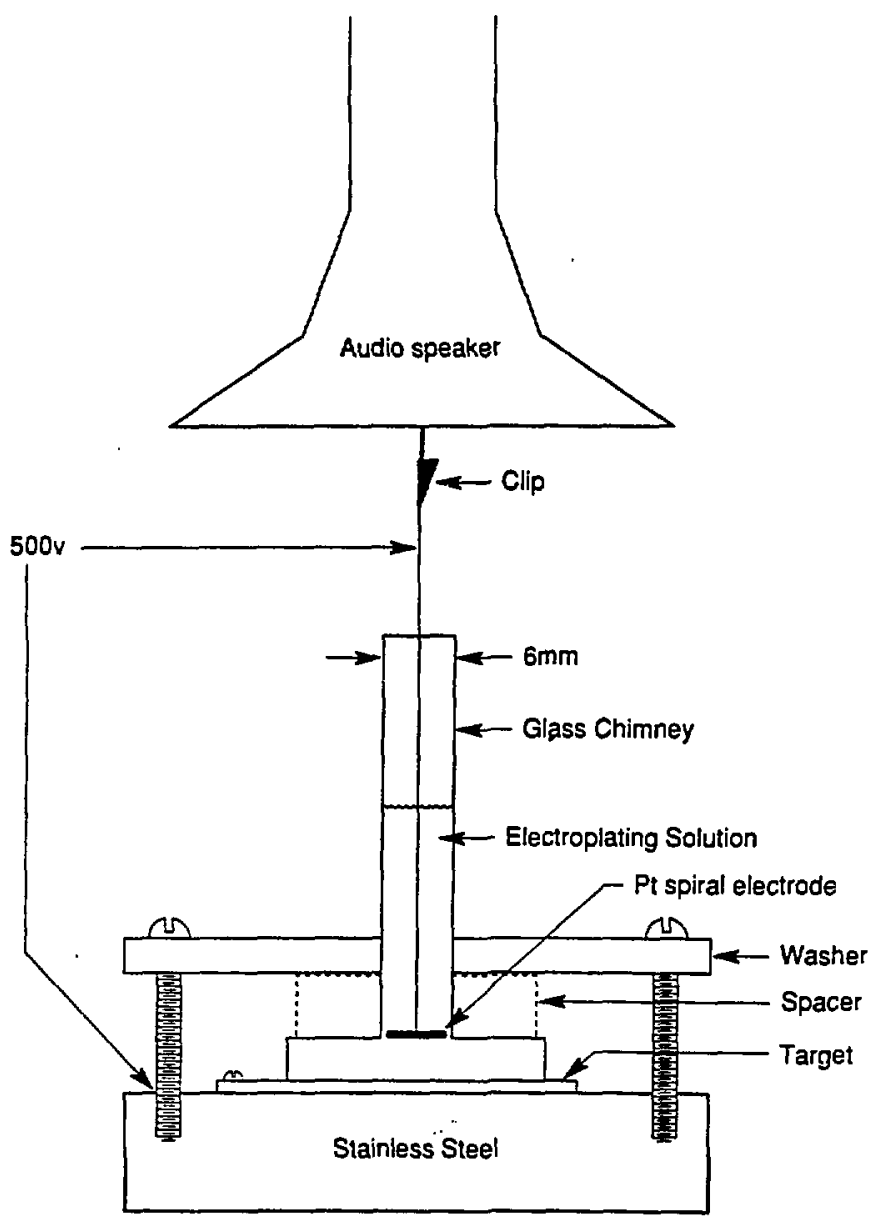

(Cutaway view of cell)

Figure A.1: Schematic of electrodeposition apparatus. 
backing case, the target was epoxied to the target holder after completion). The anode is twisted in a spiral that is parallel to the target backing material, and is connected to an audio speaker driven by a low frequency audio signal to insure application of a uniform layer of target material. In addition, the vibration of the anode provides a stirring mechanism for the solution. Care must be taken to not agitate the solution too violently or the deposition of the target material will occur in ridges, thus degrading the quality of the target. The specific chemistry and electroplating procedures for constructing ${ }^{233} \mathrm{U}$ and ${ }^{239} \mathrm{Pu}$ targets are given in the following sections. Electrodeposition from organic solutions [96] was chosen for the following advantages: (1) films between $10-1000 \mu \mathrm{g} / \mathrm{cm}^{2}$ can be produced, (2) target backings which are normally etched by acids can be used, (3) uniform depositions with good adhesion to the backings are produced, (4) electrodeposition times are short, (5) high plating efficiency is ideal for use with small quantities of material, (6) and it can be used for actinides. 


\section{A.2 ${ }^{233} \mathrm{U}$ Target Preparation}

Some initial sample preparation was required, so $\sim 1-2 \mathrm{mg}$ of ${ }^{233} \mathrm{U}$ in an unknown chen:ical form was dissolved in $2 \mathrm{ml}$ of concentrated $\mathrm{HNO}_{3}$ in a test tube. This solution was evaporated to dryness in a hot water bath with bubbling $\mathrm{N}_{2}$ gas. The uranium sample was then dissolved in a small amount of $1 \mathrm{M} \mathrm{HNO}_{3}$ and evaporated to dryness twice more to convert the uranium to the nitrate chemical form and remove other anions like $\mathrm{Cl}^{-}$, etc. The sample was then dissolved in $\sim 2 \mathrm{ml}$ isopropanol and $10 \lambda\left(1 \lambda=10^{-6} \mathrm{~L}\right)$ was removed, placed on a Pt foil, evaporated to dryness under an infra-red lamp, and counted in a low geometry $\mathrm{Si} \alpha$ detector to determine the purity of the uranium sample and the concentration of the solution. A stock solution of $\sim 30 \mu \mathrm{g} / \mathrm{ml}$ was prepared by appropriate dilution from the concentrated solution, and $1 \mathrm{ml}$ of this stock solution was placed in the electrodeposition chimney. Electroplating produced a $6 \mathrm{~mm}$ diameter spot of uranium with approxirnately $100 \mu \mathrm{g} / \mathrm{cm}^{2}$ thickness. Attempting to plate more than $100 \mu \mathrm{g} / \mathrm{cm}^{2}$ per layer results in a non-uniform layer which does not adhere and flakes from the target backing. Target backings of $\mathrm{Be}\left(2 \mathrm{mg} / \mathrm{cm}^{2}\right)$ and $\mathrm{Ni} / \mathrm{Cu}\left(135 \mu \mathrm{g} / \mathrm{cm}^{2} / 4 \mathrm{mg} / \mathrm{cm}^{2}\right)$ were used for deposition of the uranium targets. $500 \mathrm{~V}$ was applied to the cell 
for $15-20 \mathrm{~min}$, and then the target and backing was heated in a furnace at $550^{\circ} \mathrm{C}$ for $15 \mathrm{~min}$ to convert the uranium to its oxide form and expel any excess isopropanol, water, and $\mathrm{HNO}_{3}$. Attempting to plate faster (using a higher voltage) results in non-uniform layers and plating actually decreases if driven too hard. It is desirable to maintain current densities between $2-6$ $\mathrm{mA} / \mathrm{cm}^{2}$. The target was then $\alpha$-countec to determine the total thickness of the uranium deposit. The plating, heating and counting steps were repeated until the desired target thickness was achieved.

\section{A.3 ${ }^{239}$ Pu Target Preparation}

The procedure for making ${ }^{239} \mathrm{Pu}$ targets is essentially the same as that for ${ }^{233} \mathrm{U}$ targets; however, the plutonium sample was in $\sim 2 \mathrm{M} \mathrm{HClO}_{4}$ solution, so some additional chemistry was required. Concentrated $\mathrm{NH}_{4} \mathrm{OH}$ was added to the sample to make the solution basic and precipitate the plutonium. The sample was then centrifuged and the supernatant removed and discarded. The plutonium piecipitate was then washed with $\mathrm{NH}_{4} \mathrm{OH}$, centrifuged, and the supernatant removed. The remaining plutonium hydroxide sample was dissolved in concentrated $\mathrm{HNO}_{3}$ and taken to dryness in a hot water bath with 
bubbling $\mathrm{N}_{2}$. As in the uranium preparation, the plutonium is dissolved in $1 \mathrm{M} \mathrm{HNO}$ and evaporated to dryness twice more. The plutonium is then dissolved in $10 \mathrm{ml}$ isopropanol and $0.5 \mathrm{ml} 1 \mathrm{M} \mathrm{HNO}$ to avoid precipitation of the plutonium or formation of colloids. Plutonium plating efficiency was $\sim 80 \%$ due to the formation of polymer chains of plutonium which sticks to the glass walls, etc. Again a stock solution of $\sim 30 \mu \mathrm{g} / \mathrm{cm}^{2}$ was prepared. The target is electroplated as in the uranium preparation, except an initial layer of $\sim 20-30 \mu \mathrm{g} / \mathrm{cm}^{2}$ is made as a base for further layers (subsequent layers adhere more uniformly). Only $\mathrm{Ni} / \mathrm{Cu}\left(170 \mu \mathrm{g} / \mathrm{cm}^{2} / 4 \mathrm{mg} / \mathrm{cm}^{2}\right)$ backings were used. The temperature in the furnace is reduced to $\sim 250^{\circ} \mathrm{C}$ and heating is performed for $30-45 \mathrm{~min}$, to prevent the formation of a $\mathrm{Ni} / \mathrm{Cu}$ alloy in the backing. After the final electrodeposition, an additional $\mathrm{Al}$ target holder is epoxied to the target for structural support. The $\mathrm{Cu}$ is removed by dissolution in a $\mathrm{NH}_{4} \mathrm{Oll} /$ trichloroacetic acid solution forming the blue ammonia complexes of $\mathrm{Cu},\left[\mathrm{Cu}\left(\mathrm{NH}_{3}\right)\left(\mathrm{H}_{2} \mathrm{O}\right)_{5}\right]^{2+},\left[\mathrm{Cu}\left(\mathrm{NH}_{3}\right)_{2}\left(\mathrm{H}_{2} \mathrm{O}\right)_{4}\right]^{2+},\left[\mathrm{Cu}\left(\mathrm{NH}_{3}\right)_{3}\left(\mathrm{H}_{2} \mathrm{O}\right)_{3}\right]^{2+}$ and $\left[\mathrm{Cu}\left(\mathrm{NH}_{3}\right)_{4}\left(\mathrm{H}_{2} \mathrm{O}\right)_{6}\right]^{2+}$ (the addition of the fifth and sixth amine ligand is difficult in aqueous media). The $\mathrm{Ni}$ and plutonium are unaffected by the $\mathrm{NH}_{4} \mathrm{OH} / \mathrm{trichloroacetic} \mathrm{acid} \mathrm{solution.} \mathrm{A} \mathrm{more} \mathrm{thorough} \mathrm{and} \mathrm{general} \mathrm{discussion}$ of these electrodeposition procedures can be found in references [96] and [97]. 


\section{Appendix B}

\section{FITEK Program}

FITEK is a spectrum fitting code written in FORTRAN developed by Dr. Wolfgang Stöffl currently at LLNL with some debugging work and all of the documentation written by the author of this thesis. FITEK can be used to fit Gaussian or Lorentz peak shapes to almost any variety of experimental data, including exponential decay curves. In this work, FITEK was mainly used to fit peaks in $\mathrm{Ge}$ or $\mathrm{NaI}(\mathrm{Tl})$ spectra, although the PPAC angular calibration spectra were also fit using this code.

The program contains its own graphics package for display of the data on a cathode-ray tube tektronics monitor. Parameters can be input via a data file or manual cursor control. In addition to containing all of the normal spectrum manipulation features (summing spectra, differentiating spectra, subtracting spectra, multiplying spectra by a constant, display manipulation, 
two-dimensional spectral slicing, etc.), the program allows up to a six parameter background fit, possible step-function in the background, two exponential tails associated with a peak, Gaussian or Lorentz peak shapes, energy dependent parameter fits, up to a four-parameter energy calibration, automatic residual search for missed peaks, automatic peak input and automated spectra fitting (fitting similar spectra with a command file rather than manually inputting all parameters). The fitted function is expressed with the following equation:

$$
\begin{aligned}
F(c h)= & \sum_{i}\{(h g h t(i) G W)+ \\
& (1.0-T D 1)(h g h t(i) t l h 1(c h)) \\
& \exp \left[\frac{\ln (2)(c h-x(i)+\operatorname{taild} 1(c h) F W H M(c h))}{t l w 1(c h)}\right]+ \\
& (1.0-T D 2)(h g h t(i) t \operatorname{lh} 2(c h)) \\
& \exp \left[\frac{\ln (2)(c h-x(i)+t a i l d 2(c h) F W H M(c h))}{t l w 2(c h)}\right]+ \\
& \left.\int c t+\sum_{c h m x}^{c h} \operatorname{stcp}(c h)(s p e c(c h)-b c k(c h+1))\right\}
\end{aligned}
$$

where

$$
\begin{array}{r}
G W=\exp \left[\left(-\{c h-x(i))^{2}\right)\left(4 \ln (2)(F W H M(c h))^{-2}\right)\right], \\
T D 1=\exp \left[\left(-(c h-x(i))^{2}\right)\left(4 \ln (2)(F W H M(c h) \text { taild } 1)^{-2}\right)\right],
\end{array}
$$




$$
\begin{aligned}
T D 2= & \exp \left[\left(-(c h-x(i))^{2}\right)\left(4 \ln (2)(F W H M(c h) \text { taild } 2)^{-2}\right)\right] \\
f c t= & a+b(c h-c e n t e r)+c(c h-c e n t e r)^{2}+d(c h-c e n t e r)^{3}+ \\
& e(c h-c e n t e r)^{4}+f(c h-c e n t e r)^{5}
\end{aligned}
$$

and $F(c h)=$ fit function, $c h=$ channel, $i=$ number of peak, $x(i)=$ location of peak $i, h g h t(i)=$ height of peak $i, F W H M=$ full width half maximum, taild $1=$ tail distance of tail number one, taild $2=$ tail distance of tail number two, $t$ th $1, t / h 2=$ tail heights of tails number one and two, respectively, $t l w 1, t l w 2=$ tail widths of tails. number one and two, respectively, center $=$ center of fit area in channels, step $(c h)=$ stepheight at channel $c h, \operatorname{spec}(c h)=$ spectrum intensity at channel $c h, b c k(c h)=$ background intensity at channel $c h, G W=$ Gaussian width formula, $T D 1, T D 2=$ exponential tail formula for tails one and two, respectively, and $f c t=$ polynomial background function with $a, b, c, d, e$ and $f$ the fit parameters.

$F(c h)$ can be rewritten as:

$$
F(c h)=\sum_{i}\{(h g h t(i) G W)+A B+C D+E+F\}
$$

where

$$
G W=\exp \left[\left(-(c h-x(i))^{2}\right)\left(4 \ln (2)(F W H M(c h))^{-2}\right]\right.
$$




$$
\begin{aligned}
& A=(1.0-G W)(\operatorname{taild} 1(c h))^{2} h g h t(i) \operatorname{thl}(c h) \\
& B=\exp [\ln (2)(c h-x(i)+\operatorname{taildl}(c h))(F W H M(c h) / t l w 1(c h))] \\
& C=(1.0-G W)(\text { taild } 2(c h))^{2} h g h t(i) t h 2(c h) \\
& D=\exp [\ln (2)(c h-x(i)+\operatorname{taild2}(c h))(F W H M(c h) / t l w 2(c h))] \\
& E=a+b(c h-c e n t e r)+c(c h-c e n i e r)^{2}+d(c h-c e n t e r)^{3}+ \\
& e(\text { ch }- \text { center })^{4}+f(\text { ch }- \text { center })^{5} \\
& F=\sum_{c h m x}^{c h} \operatorname{step}(c h)(\operatorname{spec}(c h)-b c k(c h+1)
\end{aligned}
$$

As can be seen, the first portion of the equation is merely the equation for a Gaussian peak shape (hght(i) GW). The second portion is the equation for the first tail $(A B)$. The tail functinn is obtained by multiplying an inverted Gaussian peak shape $(A)$ and an exponential function $(B)$. This tail function is common for Ge sfectra. The third portion of the equation is the second tail function (CD). Again, it is an inverted Gaussian $(C)$ multiplied by an exponential function $(D)$. The fourth portion of the equation is the background function $(E)$. The background function is a polynomial of up to fifth order. The final portion of the equation is the step function for the background $(F)$. It is obtained from the difference between the spectrum and the background. 
The difference between the fitted function $F(c h)$ and the actual data is minimized using a multi-dimensional matrix inversion method [98]. A matrix is constructed of initial guesses for all parameters allowed to vary in the particular fit and the first partial derivatives (gradient $\nabla F(c h)$ ) are obtained. The method is to then build up a sequence of matrices $H_{i}$ such that

$$
\lim _{i \rightarrow \infty} H_{i}=A^{-1}
$$

The minimum point $x_{m}$ is given by:

$$
A \cdot x_{m}=b
$$

and at some point $\boldsymbol{x}_{\boldsymbol{i}}$

$$
A \cdot x_{i}=\nabla F\left(x_{i}\right)+b
$$

Subtracting equation B.16 from B.15:

$$
x_{m}-x_{i}=A^{-1} \cdot\left[-\nabla F\left(x_{i}\right)\right]
$$

where $x_{m}-x_{i}$ is the step to get to the minimum and $A^{-1} \cdot\left[-\nabla F\left(x_{i}\right)\right]$ is known once $H \approx A^{-1}$ is obtained recursively. The recursive relationship is then

$$
x_{i+1}-x_{i}=H_{i+1} \cdot\left[\nabla F\left(x_{i+1}\right)-\nabla F\left(x_{i}\right)\right]
$$


and there exists an updating formula for $H_{i+1}$ as a function of $H_{i},\left(x_{i+1}-x_{i}\right)$, and the gradients $\left[\nabla F\left(x_{i+1}\right)-\nabla F\left(x_{i}\right)\right]$. This algorithm is similar to rolling a bali around on a surface until a pocket or minimum is encountered.

However, the method does have some limitations and disadvantages. The number of free variables in the fit is limited, as unrestricted variation of all parameters in one fit is likely to result in singularities or some totally unphysical and meaningless result (for instance, if the tail height and tail width are both allowed to vary, one miglit wander off to $\pm \infty$ ). In addition, some reasonable initial guesses for the parameters must be used to start the iterations.

In the FITEK code, once the iterations have converged, final values and their associated errors of all parameters are displayed (both numerically and graphically) and the fit quality information is given $\left(\chi_{\nu}^{2}\right.$, local $\chi^{2}$, scatter or $\chi^{2}$, and the residuum). The residuum, or the difference between the fitted function and the data, can be differentiated and searched for the characteristic positiveand negative-spike of missed Gaussian peak shapes, and can indicate areas of the fitted region where unsatisfactory results are obtained.

Because a linear combination of errors is used in the matrix (easier to do than the norma! sum-of-squares method), the errors obtained are slightly overestimated. To get the "truc" error on a parameter of the fit, one must fix 
all other parameters to their "true" values (here the best fit is typically used to obtain the "true" values since one doesn't a prioriknow the "true" values), and then step the parameter of interest slowly fifty or one-hundred times around the "true" value monitoring the scatter for each of the fits. The scatter for the fits will trace out a parabola (symmetric or asymmetric depending on the situation) with the minimum of course being the best or "true" parameter. A scatter increase of one from the minimum is the $1 \sigma$ error, an increase of four is the $2 \sigma$ error, etc. For example, to get the error in the peak widths, one would fix all other parameters (locations, heights, background, etc.) and step the width around the "true" value and find where the scatter increased by one.

A simulation was run to determine the magnitude of the overestimation of the errors. A raw integration method was compared with FITEK fits with free parameters and FITEk fits using the scatter method described above for two ideal Gaussian peaks of width $(\sigma=5,2 \sigma=10)$ and heights of 100 counts and 10000 counts. The background was generated randomly and was between $40-50$ counts/channel. The results are shown in Table B.1.

The errors quoted in Table B.l and throughout this thesis are $1 \sigma$ errors. The difference between errors of intensities obtained from raw integration and FITEK (with free parameters) is $10-20 \%$. Additionally, the difference between 
Table B.1: Comparison of FITEK results and raw integration methods

\begin{tabular}{|c|c|c|c|c|}
\hline $\begin{array}{c}\text { Peak } \\
\text { height }\end{array}$ & $\begin{array}{c}\text { Raw int. } \\
\text { Area }- \text { blgd } \\
\pm \% \text { err }\end{array}$ & $\begin{array}{c}\text { FITEK } \\
\text { (free param.) } \\
\text { Int. } \pm \% \text { err }\end{array}$ & $\begin{array}{c}\text { FITEK } \\
\text { (free param.) } \\
\text { FWHM } \pm \text { err }\end{array}$ & $\begin{array}{c}\text { FITEK } \\
\text { (scatter meth.) } \\
\text { FWHM } \pm \text { err }\end{array}$ \\
\hline 100 & $420.4 \pm 5 \%$ & $425 \pm 6.2 \%$ & $6.765 \pm 0.589$ & $6.765 \pm 0.515$ \\
10000 & $123727.6 \pm 0.2 \%$ & $123642.8 \pm 0.3 \%$ & $11.425 \pm 0.032$ & $11.425 \pm 0.027$ \\
\hline
\end{tabular}

errors in the FITEK (free parameter) and FITEk (scatter method) fits for the FWHM is about $15-20 \%$ again. In both cases, the FITEK (free parameter) method overestimated the errors by about $15 \%$. One could correct all the error bars on fitted parameters by $15 \%$; however, in this thesis the uncorrected FITEK (free parameter) errors are given in all cases except where otherwise noted. The distinction between the widths presented in Table B.1 (FWHM) and the Gaussian widths $(2 \sigma=10)$ used as input data should be noted. 


\section{Bibliography}

[1] I.Y. Lee, D. Cline, R.S. Simon, P.A. Butler, P. Colombani, M.W. Guidry, F.S. Stephens, R.M. Diamond, N.R. Johnson, and E.Eichler Phys. Rev. Lett. 37 (1976) 420.

[2] P. Taras, D. Ward, H.R. Andrews, J.S. Geiger, R.L. Graham, and W. McLatchie Nucl. Phys. A289 (1977) 165.

[3] R.M. Diamond, B. Elbek, and F.S. Stephens Nucl. Phys. 43 (1963) 560.

[4] G.G. Seaman, E.M. Bernstein, and J.M. Palms Phys. Rev. 161 (1967) 1223.

[5] D. Ward, O. Häusser, H.R. Andrews, P. Taras, P. Skensved, N. Rud, and C. Broude Nucl. Phys A330 (1979) 225.

[6] F. Kearns, G. Varley, G.D. Dracoulis, T. Inamura, J.C. Lisle, and J.C. Willmott Nucl. Phys. A278 (1977) 109.

[7] K.A. Erb, J.E. Holden, I.Y. Lee, J.X. Saladin, and T.K. Saylor Phys. Rev. Lett. 29 (1972) 1010.

[8] B. Elbek and P.O. Tjom Adv. Nucl. Phys. 3 (1969) 259.

[9] R.A. Broglia, O. Ilansen, and C. Reidel Adv. Nucl. Phys. 6 (1973) 287.

[10] G.B. Holm, J.R. Burwell, and W.D. Miller Phys. Rev. 122 (1961) 1260.

[11] A. Isoya Phys. Rev. 130 (1963) 234.

[12] M.N. Vergnes and R.K. Sheline Phys. Rev. 132 (1963) 1736. 
[13] M.W. Sachs, V. Chasnian, and D.A. Bromley Phys. Rev. 139 (1965) B92.

[14] R.M. Bock, M. Grosse-Shulte, and W. von Oertzen Phys. Lett. 22 (1966) 456 .

[15] M.W. Guidry, R.W. Kincaid, and R. Donangelo Phys. Lett. 150B (1985) 265.

[16] J. deBoer and D.A. Bromley "Treatise on Heavy-Ion Science" vol. 1, Chapter 3 Inelastic-Scattering Coulomb Excitation (Plenum Press, New York, 1984).

[17] M.W. Guidry, T.L. Nichols, R.E. Neese, J.O. Rasmussen, L.F. Oliveira, and R.J. Donangelo Nucl. Phys. A361 (1981) 274.

[18] D. Cline Ann. Rev. Nucl. Part. Sci. 36 (1986) 683.

[19] A.O. Macchiavelli, M.A. Deleplanque, R.M. Diamond, F.S. Stephens, E.L. Dines, and J.E. Draper Nucl. Phys. A 432 (1985) 436.

[20] F.W.N. deBoer, E. Grosse, W. Spreng, H.J. Wollersheim, E.G. Eckert, and Ch. Lauterbach GSI Annual Repurt (1984).

[21] M.W. Guidry, S. Juutinen, X.T. Liu, C.R. Bingham, A.J. Larabee, L.L. Riedinger, C. Baktash, I.Y. Lee, M.L. Halbert, D. Cline, B. Kotlinski, W.J. Kernan, T.M. Sernkow, D.G. Sarantites, K. Honkanen, and M. Rajagopalan Phys. Lett. 163B (1985) 79 and references therein.

[22] P.A. Butler, C. Baktash, C.R. Binghain, M. Carpenter, D. Cline, B. Cox, M.W. Guidry, S. Juutinen, A.E. Kavka, W.J. Kernan, R.W. Kincaid, A. Larabee, I.Y. Lee, X.T. Liu, S.P. Sorensen, E. Vogt, and C.Y. Wu Phys. Lett. 191B (198T) 333 and references therein.

[23] S. Juutinen, X.T. Liu, S.P. Sorensen, B. Cox, R.W. Kincaid, C.R. Bingham, M.W. Guidry, W.J. Kernan, C.Y. Wu, E. Vogt, T. Czosnyka, D. Cline, M.L. Halbert, I.Y. Lee, and C. Baktash Phys. Lett. 192B (1987) 307.

[24] S. Juutinen PhD. thesis, University of Jyvaskyla, Jyvaskyla, Finland, 1988 (unpublished). 
[25] W.J. Kernan PhD. thesis, University of Rochester, Rochester, New York, 1989 (unpublished).

[26] X.T. Liu PhD. thesis, University of Tennessee, Knoxville, Tennessee, 1988 (unpublished).

[27] C.Y. Wu, X.T. Liu, S.P. Sorensen, R.W. Kincaid, M.W. Guidry, D. Cline, W.J. Kernan, E. Vogt, T. Czosnyka, A.E. Kavka, M.A. Stoyer, J.O. Rasmussen, and M.L. Halbert Phys. Lett. 188B (1987) 25.

[28] G. Himmele, H. Backe, P.A. Butler, D. Habs, V. Metag, H.J. Sprecht, and J.B. Wilhelmy Nucl. Phys. A404 (1983) 401.

[29] W. von Oertzen, H.G. Bohlen, B. Gebauer, R. Künkel, F. Pühlhofer, and d. Schüll Z. Phys. A326 (1987) 463.

[30] W.J. Kernan, C.Y. Wu, X.T. Liu, X.L. Han, D. Cline, T. Czosnyka, M.W. Guidry, M.L. Halbert, S. Juutinen, A.E. Kavka, R.W. Kincaid, J.O. Rasmussen, S.P. Sorensen, M.A. Stoyer, and E.G. Vogt, to be published Nucl. Phys. A (1990).

[31] W. von Oertzen, B. Gebauer, A. Gamp, H.G. Bohlen, F. Busch, and C. Schüll Z. Phys. A313 (1983) 189.

[32] K. Sapotta, R. Bass, V. Hartmann, H. Noll, R.E. Renfordt, and K. Stelzer Phys. Rev. C31 (1985) 1297.

[33] J.O. Rasmussen, M.W. Guidry, and L.F. Canto in "Proceedings of the Niels Bohr Centennial Conference", ed. by J. Bang and J. deBoer (North Holland, Amsterdam, 1985) 167.

[34] A.E. Kavka $\mathrm{PhD}$. thesis, Acta Universitatis Upsaliensis, Uppsala, Sweden, 1999 (unpublished).

[35] G.J. Gyapong, R.H. Spear, M.T. Esat, M.P. Fewell, A.M. Baxter, and S.M. Burnett Nucl. Phys. A458 (1986) 165.

[36] T. Czosynka, D. Cline, L. Hasselgren, C.Y. Wu, R.M. Diamond, H. Kluge, C. Roulet, E.K. Hulet, R.W. Lougheed, and C. Baktash Nucl. Phys. A458 (1986) 123. 
[37] Ch. Lauterbach, J. deBoer, Ch. Mittag, F. Riess, Ch. Schandera, Ch. Briançon, A. Lefebvre, S. Hlavač, and R.S. Simon Phys. Lett. B140 (1984) 187.

[38] W. Spreng, F. Azgui, H. Emling, E. Grosse, R. Kulessa, Ch. Michel, D. Schwalm, R.S. Simon, H.J. Wollersheim, M. Mutterer, J.P. Throbald, M.S. Moore, N. Trautmann, J.L. Egido, and P. Ring Phys. Rev. Lett. 51 (1983) 1522.

[39] R. Kulessa, R.P. DeVito, H. Emling, E. Grosse, D. Schwalm, R.S. Simon, A. Lefebvre, Ch. Briançon, R.J. Walen, G. Seitten, and Th.W. Elye Z. Phys. A312 (1983) 135.

[40] R.B. Piercey, J.H. Hamilton, A.V. Ramayya, H. Emling, P. Fuchs, E. Grosse, D. Schwalm, H.J. Wollersheim, N. Trautmann, A. Faessler, and M. Ploszajczak Phys. Rev. Lett. 46 (1981) 415.

[41] K. Hardt, P. Schüler, C. Günther, J. Recht, and K.P. Blume Z. Phys. A314 (1983) 83.

[42] K. Hardt, P. Schüler, C. Gūnther, J. Recht, and K.P. Blume Nucl. Phys. A407 (1983) 127.

[43] M. Jääskeläinen, D.G. Sarantites, R. Woodward, F.A. Dilmanian, J.T. Hood, and R. Jääskeläinen Nucl. Inst. Methods 204 (1983) 385.

[44] E. Grosse, A. Balanda, H. Emling, F. Folkmann, P. Fuchs, R.B. Piercey, D. Schwalm, R.S. Simon, and H.J. Wollersheim Phys. Scr. 24 (1981) 337.

[45] R.S. Simon, R.P. Devito, H. Emling, R. Kulessa, Ch. Briançon, and A. Lefebvre Phys. Lett. B108 (1982) 87.

[16] H. Ower, Th. W. Elze, J. Idzko, K. Stelzer, E. Grosse, H. Emling, P. Fuchs, D. Schwalm, H.J. Wollersheim, N. Kaffrell, and N. Trautmann Nucl. Phys. A388 (1982) 421.

[47] Nuclear Data Sheets 59 (January 1990) 270 and J. deBettencourt, C. Briancon, J.R. Tibeaux, R.J. Walen, M. Jacmar, A. Gizon, and S. Andre Bull. Acad. Sci. USSR 40 (1976) 14. 
[48] J.L. Egido, and P. Ring Nucl. Phys. A423 (1984) 93.

[49] J. Helgesson and I. Hamamoto Phys. Scr. 40 (1989) 595.

[50] P. Mōller and J.R. Nix At. Data Nucl. Data Tables 39 (1988) 213; and P. Möller and J.R. Nix LANL Preprint LA-JR-86-3983, Nov. 7, 1986.

[51] P. Ring and H.J. Mang Phys. Rev. Lett. 33 (1974) 1174.

[52] D.R. Inglis Phys. Rev. 96 (1954) 1059.

[53] K. Kumai and M. Baranger Nucl. Phys. A110 (1968) 529.

[54] R. Bengtsson and S. Frauendorf Nucl. Phys. A327 (1979) 139.

[55] A. Bohr and B. Mottelson in Nuclear Structure, vol Il (W. A. Benjamin, Inc., Massachusetts, 1975) pp. 31.

[56] J.O. Rasmussen, J. Alonso, W. Ribbe, I. Rezanka, and R. Needham LBL NSD Annual Report (1972) 163, T. Tamura, I. Rezanka, S. Iwata, J.O. Rasmussen, and J. Alonso Phys. Rev. C8 (1973) 2425, and K. Krien, R.A. Naumann, J.O. Rasmussen, and I. Rezanka Nucl. Phys. A209 (1973) 572.

[57] J. Alonso, H. Bakhru, F.M. Bernthal, J. Boutet, B. Olsen, I. Rezanka, and J.O. Rasmussen Nucl. Phys. A160 (1971) 193.

[58] S.W. Yates, I.Y. Lee, N.R. Johnson, E. Eichler, L.L. Riedinger, M.W. Guidry, A.C. Kahler, D. Cline, R.S. Simon, P.A. Butler, P. Colombani, F.S. Stephens, R.M. Diamond, R.M. Ronningen, R.D. Hichwa, J.H. Hamilton, and E.L. Robinson Phys. Rev. C21 (1980) 2366.

[59] R. Bengtsson and S. Frauendorf Nucl. Phys. A327 (1979) 139.

[60] I. Hamamoto Proceedings of the Conference on Nuclear Structure in the 90's, ORNL April 23-27, 1990, and I. Hamamoto and B. Mottelson Phys. Lett. B127 (1983) 281.

[61] W.Z. Venema, J.F.W. Jansen, R.V.F. Janssens, and J. Van Klinken Phys. Lett. B156 (1985) 163. 
[62] K. Helmer, D. Cline, M.A. Deleplanque, R. Diamond, M.W. Guidry, A.E. Kavka, W.J. Kernan, X.T. Liu, R. McDonald, A. Machiavelli, J.O. Rasmussen, F. Stephens, M.A. Stoyer, E. Vogt, and C.Y. Wu, to be published.

[63] P. Marmier and E. Sheldon in Physics of Nuclei and Particles, vol II (Academic Press, New York, 1970) p. 1322, eqs. (15-290) and (15-291).

[64] E.M. Bernstein, and J. de Boer Nucl. Phys. 18 (1960) 40.

[65] P. Marmier and E. Sheldon, Ibid, p. 1320, eqs. (15-279).

[66] K.E.G. Löbner, M. Vetter, and V. Hönig Nucl. Data Tables A7 (1970) 495.

[67] G.H. Fuller and V.W. Cohen Nucl. Data Tables A5 (1969) 433.

[68] Table of Isotopes, 7th ed., ed. C.M. Lederer and V.S. Shirley (John Wiley and Sons, New York, 1978) p. 1428.

[69] R.R. Chasman, I. Ahmad, A.M. Friedman, J.R. Erskine Rev. Mod. Phys. 49 (1977) 833.

[70] J.O. Newton Nucl. Phys. 5 (1958) 218.

[71] G. Breit and M.E. Ebel Thys. Rev. 103 (1956) 679.

[72] J.O. Rasmussen, M.A. Stoyer, M.W. Guidry, L.F. Canto and R. Donangelo "Proceedings from the International Symposium on Symmetries and Nuclear Structure”, Nuclear Science Research Conference Series, Vol. 13, Dubrovnik, Yugoslavia, June 5-14, 1986, pp. 391.

[73] K. Alder and A. Winther "Coulomb Excitation" (Academic Press, New York, 1966).

[74] C. Price, H. Esbensen, and S. Landowne Phys. Lett. 197B (1987) 15.

[75] M.J. Rhoades-Brown, R. Donangelo, M.W. Guidry, and R.E. Neese Phys. Rev. C24 (1981) 2747.

[76] H. Massmann and J.O. Rasmussen Nucl. Phys. A243 (1975) 55. 
[77] M.W. Guidry, H. Massmann, R. Donangelo, and J.O. Rasmussen Nucl. Phys. A247 (1976) 183.

[78] R. Donangelo, M.W. Guidry, J.P. Boisson, and J.O. Rasmussen Phys. Lett. 64B (1976) 337.

[79] L.F. Canto, R.J. Donangelo, J.O. Rasmussen, P. Ring, and M.A. Stoyer Phys. Lett. B248 (1990) 10.

[80] L.F. Canto, R.J. Donangelo, M.W. Gujdry, A.R. Farhan, J.O. Rasmussen, P. Ring, and M.A. Stoyer Phys. Lett. 241B (1990) 295.

[81] J. deBoer, C.H. Dasso, and A. Winther Z. Phys. A335 (1990) 199.

[82] C.H. Dasso and A. Winther Phys. Lett. 242B (1990) 323.

[83] K. Alder, Aa. Bohr, T. Huus, B. Mottelson and Aa. Winther Rev. Mod. Phys. 28 (1956) 432.

[84] R. Donangelo PhD. thesis, University of California, Berkeley, CA, 1977 (unpublished).

[85] L.F. Canto, R. Donangelo, R.S. Nikan, and P. Ring Phys. Lett. 192B (1987) 4.

[86] S. Juutinen, X.T. Liu, S.P. Sorensen, B. Cox, R.W. Kincaid, C.R. Bingham, M.W. Guidry, W.J. Kernan, C.Y. Wu, E. Vogt, D. Cline, M.L. Halbert, 1.Y. Lee, and C. Baktash "Progress Report on Nuclear Spectroscopic Studies", Department of Physics and Astronomy, University of Tennessee, Knoxville, TN Feb. 1986, 87.

[87] A. Winther and J. deBoer "A Computer Code for Multiple Coulomb Excitation", Caltech report (Nov. 1965), reference 8, reprinted in reference [87].

[88] L.F. Canto, R.J. Donangelo, A.R. Farhan, M.W. Guidry, J.O. Rasmussen, P. Ring, and M.A. Stoyer Symposium on Exotic Nuclear Spectroscopy, American Chemical Society (Miami Beach, Oct. 1989).

[89] K. Helmer PhD. thesis, University of Rochester, Rochester, NY, in progress. 
[90] R.S. Nikam and P. Ring Phys. Rev. Lett. 58 (1987) 980.

[91] M.V. Berry Proc. R. Soc. (London) A392 (1984) 15.

[92] R.S. Nikam et al. Z. Phys. A234 (1986) 241; Phys. Lett. B185 (1987) 269.

[93] J.O. Rasmussen, M.A. Stoyer, L.F. Canto, R. Donangelo and P. Ring "Nuclear Rotational Population Patterns in Heavy-Ion Scattering and Transfer Reaction $3^{\prime \prime}$, to be published Z. Phys. (1990).

[94] B.R. Mottelson and S.G. Nilsson Mat. Fys. Skr. Dan. Vid. Selsk. 1 (1959) no. 8 and S.G. Nilsson Mat. Fys. Medd. Dan. Vid. Selsk. 2 (1955) no. 16.

[95] P.A. Butler, D. Cline, C.Y. Wu, R. Ibbotsen, K. Helmer, M. Devlin, R.J. Poynter, I.Y. Lee, J.D. Garrett, M.W. Guidry and X.T. Liu "Second Minimum Spectroscopy Using Heavy-Ion Transfer Reactions" ORNL proposal, June 1990.

[96] D.C. Aumann and G. Müllen Nucl. Inst. Methods 115 (1974) 75.

[97] J.E. Evans, R.W. Lougheed, M.S. Coops, R.W. Hoff and E.K. Hulet Nucl. Inst. Methods 102 (1972) 389.

[98] W.H. Press, B.P. Flannery, S.A. Teukolsky and W.T. Vetterling "Numerical Recipes - The Art of Scientific Computing" Cambridge Univ. Press, Cambridge (1986) $307 \mathrm{ff}$. 